\title{
MINIMUM CELL SIZE FOR INFORMATION CAPACITY INCREASE IN CELLULAR WIRELESS NETWORK
}

\section{KWASHIE AMARTEI ANANG}

A thesis submitted in partial fulfilment of the requirements of the University of Greenwich for the Degree of Doctor of Philosophy

\section{April 2013}




\section{DECLARATION}

I certify that this work has not been accepted in substance for any degree, and is not concurrently being submitted for any degree other than that of Doctor of Philosophy $(\mathrm{PhD})$ being studied at the University of Greenwich. I also declare that this work is the result of my own investigations except where otherwise identified by references and that I have not plagiarized the work of others.

Signed $\quad$...................................., Date

Kwashie Amartei Anang

(Student)

Signed $\quad$......................................, Date

Prof. Predrag Rapajic

(Ist supervisor)

Signed $\quad$...................................., Date

Dr. Ruiheng Wu

(2nd supervisor). 


\section{ACKNOWLEDGMENTS}

My endeavours, within and beyond this research work over the past three years, would have not been successful without the support of people who believed in me. I thank the Almighty God, who created the heaven and earth for bringing me this far, for it is not by my might nor by my power, but by His grace and spirit. The Lord says we should thank Him in every situation; hence to Him be the glory and thanks.

One could hardly hope for a better mentor than Professor Predrag B. Rapajic. I am deeply grateful and thankful to Professor Predrag B. Rapajic for his endless support, guidance, and encouragement. Professor Predrag B. Rapajic's dedication to research and the breadth and depth of his knowledge on all things scientific have greatly amazed me and others both in his research group and outside. Researchers who followed this role model have become good researchers and engineers. Regardless of where I go, I have learned from Predrag that a true and genuine love and dedication to one career is what it takes to excel in every environment.

I thank Dr. Ruiheng Wu for his support as my second supervisor, not forgetting Dr. Kao-Cheng Huang and Dr. Charan Litchfield for their contributions, support, criticisms, strong encouragement and advice throughout the years of this research work. I also thank Professor Ndy Ekere, Dr. Steve Woodhead, and Dr. Rajinderpal Bhatti for their administrative support and assistance over the past years. I would also like to thank David Derrington for his feedback on the structure of the thesis.

This work has benefit from valuable discussions with friends and colleagues at the University of Greenwich, including Dr. Triantafyllos (Aldo) Kanakis, Dr. Titus I. Eneh, Dr. Athar Qureshi, Yogesh Nijsure, Lawal Bello, Andrew A. Adekunle, and Grace Oletu. I must also acknowledge fellow PhD students whose contributions made the years 
of graduate school enjoyable: Peter K. Bernasko, Emeka Amalu, Kenny C. Otiaba and Mathias Ekpu. I'm also grateful to the supporting staff of the school of engineering at Medway for their friendly and skilled assistance. My gratitude also goes to the school of Engineering, for the provision of financial assistance as without this assistance this research work would not have been possible.

My gratitude also goes to my wife Hilda Marteki Dinsin for her support during this research work and special appreciation to my lovely kids (Nii Amarh, Naa Amarteley and Naa Djanwa) for their disturbance, laughter, and friendship, which brightened every moment of my life through graduate school.

I also thank all my aunties for their financial support and encouragement, not forgetting my cousin and wife, Prof and Mrs. Gilford A. Ashitey for their words of encouragement. Special thanks goes to my only sister Cecilia Amarley, for her encouragement, support and her willingness to go extra mile to get whatever I needed to make this dream come true, to my brothers Andrew and Kwashie I say big thanks for the support and encouragement. I'm grateful to the pastor and members of Jesus Revival Ministry International in the UK, for their prayers, encouragement, and support, which made it possible for me to see the light at the end of the tunnel.

Finally, my most heartfelt gratitude goes to the two most special people in my life. My father and mother, Mr. and Mrs. Clement M. Anang, they supported me with endless love, caring, pride and they also inspired me with a love of learning and academia. I thank them both for always being there for me. 


\section{ABSTRACT}

In conventional cellular wireless communication system, interference modelling has focused on the six primary co-channel interfering cells (first tier co-channel cells). In the current accepted interference model, co-channel interfering cells beyond the first tier (subsequent tier co-channel cells) are neglected. This currently accepted interference models is suitable for cellular wireless communication systems operating at carrier frequencies, $f_{c}$ $=0.9$ and $1.8 \mathrm{GHz}$, cell size radii $R>1 \mathrm{~km}$ and basic path loss exponent $\alpha \geq 2$. The future and emerging wireless communication systems are expected to be operating at frequen$\operatorname{cies} f_{c}>2 \mathrm{GHz}(3.35-15.75 \mathrm{GHz})$, cell size radii $R \leq 1 \mathrm{~km}$ and basic path loss exponent $\alpha \leq 2$. This, makes the current acceptable co-channel interference model unsuitable for information capacity analysis of the future cellular systems. Therefore, a co-channel interference model suitable for future and emerging wireless communication system becomes necessary.

In this thesis a new and modified interference model is proposed. The proposed interference model includes the first and subsequent tier co-channel interfering cells. The proposed interference model will be suitable for cellular wireless communication systems operating at carrier frequencies $f_{c}>2 \mathrm{GHz}$, cell size radii $R \leq 1 \mathrm{~km}$ and basic path loss exponent $\alpha \leq 2$. A mathematical analysis, supported by computer simulation is used, to study the uplink information capacity performance for the conventional and proposed interference model. The analysis and simulation results of the proposed interference model show that at carrier frequencies $f_{c}>2 \mathrm{GHz}$, co-channel interfering cells beyond the first tier become active as cell size radius $R$, reduces. As an example for a carrier frequency $f_{c}$ $=15.75 \mathrm{GHz}$, cell size radius $R=100 \mathrm{~m}$ at a normalized reuse distance $R_{u}=4$, there was a $15.32 \%$ decrease in the information capacity between the conventional and proposed 
interference model.

An information capacity - cost analysis is used to find a minimum cell size for information capacity increase in cellular wireless network, thus a theoretical limit to cell size reduction. The results show that as the cell size radius $R$ reduces to $300 \mathrm{~m}$ and less, the proposed interference model show a $5.76-18.89 \%$ decrease in the information capacity per unit cost $\left(£, \$\right.$, etc) at microwave carrier frequencies $f_{c}>3.35 \mathrm{GHz}$. This result illustrates that there is a theoretical limit to cell size reduction in relation to information capacity performance and cost.

An inductive approach is used to generate a formula for calculating the number of co-channel interfering cells $N_{n}$ in a cellular wireless site layout. Such a formula allows one to calculate the number of co-channel interfering cells in subsequent tiers of a cellular wireless site layout. The geometric derivation shows that the number of co-channel interfering cell $N_{n}$ in a subsequent tier is the product of the number of co-channel interfering cells in the first tier $N_{I}$ and the tier number $n$. Thus, the number of co-channel interfering cell in a subsequent tier $N_{n}=N_{I} \times n$. This formula enables subsequent tier co-channel interference to be included in the information capacity analysis of future and emerging, and finding the minimum cell size for information capacity increase in a cellular wireless communication system. 


\title{
LIST OF ABBREVIATIONS
}

\author{
ACI Adjacent Channel Interference \\ AMPS Advanced Mobile Phone Services \\ ASE Area Spectral Efficiency \\ AWGN Additive White Gaussian Noise \\ BER Bit Error Rate \\ BS Base Station \\ BTS Base Transceiver Station \\ CAPEX Capital Expenditures \\ CDMA Code Division Multiple Access \\ CCI Co-channel Interference \\ CIR Carrier-to-Interference Power Ratio \\ CMR Cellular Mobile Radio \\ CNR Carrier-to-Noise Ratio \\ $\mathrm{dBm} \quad$ Power ratio in decibels of the measured power referenced to one milliwatt \\ EMI Electromagnetic Interference \\ FDD Frequency Division Duplex \\ FDMA Frequency Division Multiple Access \\ FFR Fractional Frequency Reuse \\ FM Frequency Modulation \\ $1 \mathrm{G} \quad$ First-Generation Mobile System \\ 2G Second-Generation Mobile System \\ 3G Third-Generation Mobile System
}




\begin{tabular}{|c|c|}
\hline $4 \mathrm{G}$ & Fourth-Generation Mobile System \\
\hline $5 \mathrm{G}$ & Fifth-Generation Mobile System \\
\hline 3GPP & 3rd Generation Partnership Project \\
\hline GPS & Satellite Navigation Syetm \\
\hline GSM & Groupè Spècial Mobile/Global System for Mobile Communications \\
\hline HetNet & Heterogeneous networks \\
\hline IMTS & Improved Mobile Telephone Services \\
\hline LOS & Line-of-Sight \\
\hline LTE & Long Term Evolution for Wireless Communication \\
\hline LTE - Advanced & Long Term Evolution - Advanced for Wireless Communication \\
\hline MS & Mobile Station \\
\hline MSC & Mobile Switching Centre \\
\hline MIMO & Multiple-Input Multiple-Output \\
\hline Non-Los & Non-Line-of-Sight \\
\hline OFDM & Orthogonal Frequency Division Multiplexing \\
\hline PSTN & Public Switched Telephone Network \\
\hline RAN & Radio Access Network \\
\hline RF & Radio Frequency \\
\hline RFI & Radio Frequency Interference \\
\hline RNC & Radio Network Controller \\
\hline $\mathrm{Rx}$ & Receiver \\
\hline SHF & Super-High-Frequency Band \\
\hline SINR & Signal-to-Interference Noise Ratio \\
\hline SNR & Signal-to-Noise Ratio \\
\hline TDD & Time Division Duplex \\
\hline TDMA & Time Division Multiple Access \\
\hline Tx & Transmitter \\
\hline UHF & Ultra High Frequency \\
\hline WLANS & Wireless Local Area Networks \\
\hline
\end{tabular}




\title{
LIST OF NOTATION SYMBOLS
}

\author{
$\alpha \quad$ Distance dependent basic path loss exponent \\ $\rho \quad$ Distance dependent extra path loss exponent \\ $A_{e} \quad$ Area spectral efficiency [bits $\left./ \mathrm{sec} / \mathrm{Hz} / \mathrm{km}^{2}\right]$, \\ $C_{k} \quad$ Maximum data rate of the kth user [bits/sec] \\ $B S_{\mathcal{C}} \quad$ Cost per BS $[£, \$$, etc $]$ \\ $D \quad$ Co-channel reuse distance $[\mathrm{m}]$ \\ $f_{c} \quad$ Carrier frequency $[\mathrm{Hz}]$ \\ $g$ breakpoint distance for two-slope path loss model [m] \\ $h_{m} \quad$ Mobile station antenna height [m] \\ $h_{b} \quad$ Base station antenna height $[\mathrm{m}]$ \\ $K \quad$ Constant path loss factor \\ $P_{d} \quad$ Desired signal power [W] \\ $P_{t} \quad$ Transmitted signal power [W] \\ $P_{r} \quad$ Received signal power [W] \\ $P_{f i} \quad$ Interference power from first tier co-channel cell [W] \\ $P_{s i} \quad$ Interference power from second tier co-channel cell [W] \\ $P_{i j} \quad$ Interference power from the jth tier co-channel cell [W] \\ $P_{I} \quad$ Total interference signal power [W] \\ $N_{c} \quad$ Cluster size [integer] \\ $N_{s} \quad$ Total number of active services channels per cell [integer] \\ $N_{B S} \quad$ Number of base stations [integer] \\ $N_{m} \quad$ Thermal noise power [W]
}




$\begin{array}{ll}N_{F I} & \text { Number of interfering co-channel cells in the first tier [integer] } \\ N & \text { Noise power [W] } \\ N_{S I} & \text { Number of interfering co-channel cells in the second tier [integer] } \\ N_{n} & \text { Number of interfering co-channel cells in the nth tier [integer] } \\ N_{T} & \text { Total number of interfering co-channel cells [integer] } \\ r & \text { Communication distance between desired MS and reference BS [m] } \\ R_{u} & \text { Normalized reuse distance [m] } \\ R & \text { Radius of cells coverage (cell size radius) [m] } \\ R_{0} & \text { Minimum distance a MS can be from BS antenna [m] } \\ S & \text { Signal power [W] } \\ T_{\mathcal{C}} & \left.\text { Total infrastructure cost [unit cost } / \mathrm{km}{ }^{2}\right] \\ W & \text { Total allocated bandwidth per cell [Hz] } \\ W_{k} & \text { Bandwidth allocated to the kth user [Hz] } \\ \lambda & \text { Carrier wavelength [m] } \\ \gamma & \text { Carrier-to-interference ratio } \\ p_{r}, \theta(r, \theta) & \text { Probability distribution function (PDF) of a mobile location relative to a BS } \\ p_{\gamma}(\gamma) & \text { Probability distribution function (PDF) of the average mean CIR } \\ (x, \theta) & \text { Polar coordinates for desired and interfering MSs }\end{array}$




\section{CONTENTS}

DECLARATION I

ACKNOWLEDGMENTS ii

ABSTRACT iv

LIST OF ABBREVIATIONS vi vi

LIST OF NOTATION SYMBOLS viii

LIST OF FIGURES XV X

LIST OF TABLES X xxi

LIST OF PhD CANDIDATE'S PUBLICATIONS RELATED TO PhD THESIS xxii

$\begin{array}{lll}\text { Chapter } 1 & \text { Introduction } & 1\end{array}$

1.1 Research Aims of the Thesis .................. 2

1.2 Wireless Communication ................... 2

1.2.1 Electromagnetic Waves Propagation and Interference . . . . . . . 3

1.2.2 History of Cellular Wireless Communications and Influence of Co-channel Interference . . . . . . . . . . . . . . 4

1.3 Possible Future Development Direction of Cellular Wireless Communi-

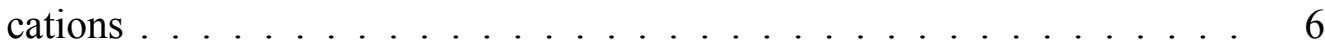

1.4 Technical Issues Related to the Future of Wireless Communication . . . . 8

1.4.1 Spectral Efficiency . . . . . . . . . . . . . 12

1.4.2 Interference in Cellular Wireless Systems . . . . . . . . . . . . 12 
1.5 Motivation for the Research Work in the Thesis . . . . . . . . . . . . . 14

1.5.1 Contributions of the Thesis . . . . . . . . . . 15

1.6 Organization of the Thesis . . . . . . . . . . . . . . . 19

1.6.1 Outline of the Thesis . . . . . . . . . . . . . 19

Chapter 2 Overview of Cellular Wireless System 21

2.1 The Cellular Wireless Communication Concept . . . . . . . . . . . . . 22

2.1.1 Frequency Reuse Concept in Cellular System . . . . . . . . . . 25

2.2 Properties of Cellular Wireless Geometry . . . . . . . . . . . . . . 26

2.3 Wireless Channels . . . . . . . . . . . . . . . 26

2.3.1 Additive Noise Channels . . . . . . . . . . . . . . . . . . 27

2.3.2 Multipath Signal Propagation Channels . . . . . . . . . . . 28

2.4 Propagation Losses $\ldots \ldots \ldots$

2.4.1 Ray-Tracing Technique . . . . . . . . . . . . . 30

2.4 .2 Two-Path Model . . . . . . . . . . . . . . . . . . 31

2.4.3 Statistical Fading Models . . . . . . . . . . . . . . . 34

2.4.4 Site-Specific Models for Path Loss . . . . . . . . . . . . . . 35

2.5 Cellular Wireless Channels . . . . . . . . . . . . . . . . . . . 36

2.5.1 Macrocells Wireless Channel _. . . . . . . . . . . 38

2.5.2 Microcells Wireless Channel . . . . . . . . . . . . . . . 39

2.6 Interference in Cellular Wireless Systems _ . . . . . . . . . . . . 39

2.7 Cellular Wireless System Information Capacity . . . . . . . . . . . . 41

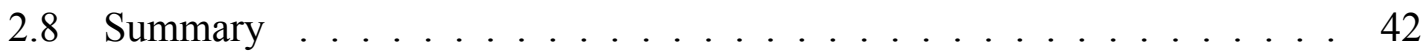

\section{Chapter 3 Proposed Formula to Calculate Number of Co-channel Interfering} Cells in Cellular System Site Layout, and Co-channel Interference Model for Two Tier Co-channel Cells $\quad 43$

3.1 Introduction . . . . . . . . . . . . . . . . . . . 44

3.2 Frequency Reuse for Spectrum Allocation . . . . . . . . . . . . . . 46

3.2.1 Cellular Wireless System Site Layout . . . . . . . . . . . . . 48

3.2.2 Proposed formula for Calculating Number of Co-channel Cells . . 53

3.3 Signal and Interference Model for Emerging Cellular Wireless Networks . 58 
3.3.1 System Model for LOS Propagation Environment . . . . . . . . . 59

3.3.2 Relationship between Breakpoint Distance and Received Signal . 60

3.3.3 Modified Breakpoint Distance for Higher Carrier Frequency . . . 64

3.3.4 Cellular Wireless System Interference . . . . . . . . . . . . . 65

3.3.5 Conventional Cellular Wireless Uplink Interference Model . . . . 67

3.4 Proposed Two Tier Uplink Interference Model . . . . . . . . . . . . . . . 68

3.5 Summary .......................... 70

Chapter 4 Information Capacity Performance Analysis for Two Tier Co-channel

$\begin{array}{ll}\text { Interference } & \mathbf{7 2}\end{array}$

4.1 Introduction . . . . . . . . . . . . . . . . 73

4.2 Two - Tier Co-channel Interference Model . . . . . . . . . . . . . . . . 76

4.2.1 Mobile and Interfering Users Distribution in Cells . . . . . . . 76

4.3 System Model for Information Capacity Analysis . . . . . . . . . . . . . 78

4.3.1 Area Spectral Efficiency - Conventional . . . . . . . . . . . . 78

4.3.2 Area Spectral Efficiency for Two-Tier Interference Model . . . . 79

4.4 Simulation Model-Information Capacity Comparison . . . . . . . . . . . 81

4.4.1 Simulation Setup for One and Two Tier Interference Model . . . 83

4.5 Numerical Results-Information Capacity Comparison . . . . . . . . . . . 85

4.5.1 Effect of Basic Path Loss Exponent-Two Tier Interference . . . . 91

4.5.2 Effect of Extra Path Loss Exponent-Two Tier Interference . . . . 98

4.5.3 Effect of Base Station Antenna Height-Two Tier Interference . 105

4.5.4 Effect of Vehicular Traffic-Two Tier Interference . . . . . . . . 108

4.6 Summary . . . . . . . . . . . . . . . . . . 113

Chapter 5 Proposed Multiple Tier Co-channel Interference Model and Information Capacity Performance Analysis for Multiple Tier Co-channel Inter$\begin{array}{lr}\text { ference } & 114\end{array}$

5.1 Introduction . . . . . . . . . . . . . . . . 115

5.2 Multiple Tier Interference General System Model . . . . . . . . . . . . . 118

5.2.1 Relationship between Breakpoint Distance, Carrier Frequency and Number of Co-channel Tiers . . . . . . . . . . . . . . . 119 
5.2.2 Relationship between Breakpoint Distance, Cell Size Radius and Number of Co-channel Tiers . . . . . . . . . . . . . . . 125

5.2.3 Mobile and Interfering Users Distribution in Cells . . . . . . . 128

5.3 Multiple Tier Interference Modelling . . . . . . . . . . . . . . . 128

5.3.1 General Interference Model _ . . . . . . . . . . . . . . 128

5.3.2 Proposed Model - Multiple Tier Interference . . . . . . . . . . . 129

5.4 Information Capacity - Multiple Tier Interference . . . . . . . . . . . 130

5.4.1 Tiers of Co-channel Interfering Cell for Consideration in Smaller Cell Size Radius System . . . . . . . . . . . . . . . . 132

5.5 Propagation/System Parameter Impact Analysis ～. . . . . . . . . . . . 133

5.6 Numerical Simulation Setup for Propagation/System Parameter Impact Analysis . . . . . . . . . . . . . . . . . . . 134

5.7 Numerical Results . . . . . . . . . . . . . . . . . . . . . . . . . 137

5.7.1 Impact of Basic Path Loss Exponent-Multiple Tier Interference 149

5.7.2 Impact of Extra Path Loss Exponent-Multiple Tier Interference 159

5.7.3 Impact of Antenna Height-Multiple Tier Interference . . . . . . 165

5.7.4 Impact of Vehicular Traffic - Multiple Tier Interference . . . . 168

5.8 Summary . . . . . . . . . . . . . . . . . . 173

\section{Chapter 6 Minimum Cell Size for Information Capacity Increase in Cellular} $\begin{array}{lr}\text { Wireless Network } & 175\end{array}$

6.1 Introduction . . . . . . . . . . . . . . . . . . . 176

6.2 System Model for Finding Minimum Cell Size Radius . . . . . . . . . 178

6.2.1 Multiple Tier Co-channel Interference Model . . . . . . . . . . . 178

6.3 Cost Estimation System Model . . . . . . . . . . . . . . . . . . . . . . . 179

6.3.1 Infrastructure Cost . . . . . . . . . . . . . . . 179

6.4 Information Capacity/Cost Performance Analysis . . . . . . . . . . . . 181

6.5 Numerical Simulations for Information Capacity/Cost . . . . . . . . . . 185

6.5.1 Numerical Simulations Setup . . . . . . . . . . . . . 185

6.6 Numerical Results . . . . . . . . . . . . . . . . . . . . 185

6.6.1 Information Capacity/Cost Ratio Numerical Results . . . . . . 186 
6.7 Wireless Network . . . . . . . . . . . . . . . . . . . . . 194

6.7.1 Current Cellular Mobile Communication Technologies . . . . . . 194

6.7.2 Heterogeneous Networks . . . . . . . . . . . . . . . . 195

6.8 Fractional Frequency Reuse _ . . . . . . . . . . . . . . . . 197

6.9 Possible Impact of Fractional Frequency Reuse on the Minimum Cell Size

Radius . . . . . . . . . . . . . . . . . . . . . 199

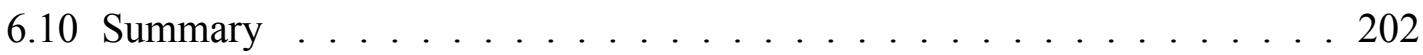

$\begin{array}{lll}\text { Chapter } 7 & \text { Conclusions and Future Work } & 204\end{array}$

7.1 Conclusions . . . . . . . . . . . . . . . 205

7.2 Future Work . . . . . . . . . . . . . . . . . 206

$\begin{array}{ll}\text { Bibliography } & \mathbf{2 1 0}\end{array}$ 


\section{LIST OF FIGURES}

1.1 Basic wireless communication system. . . . . . . . . . . . . . 3

1.2 The future development for wireless communication systems . . . . . . . 7

1.3 Technical challenges to be addressed in implementing the wireless vision. 8

1.4 Wireless communication channel. . . . . . . . . . . . . . . . 10

1.5 Wireless communications radio spectrum allocation and usage . . . . . . 11

1.6 Conventional cellular wireless system. . . . . . . . . . . . . . . 13

1.7 Road map of the thesis and thesis contributions. . . . . . . . . . . . 18

2.1 A cellular wireless network. . . . . . . . . . . . . . . . 24

2.2 Basic additive Gaussian noise channel. . . . . . . . . . . . . . . . 27

2.3 Distribution of single diffraction, reflection and scatters in a wireless communication environment. . . . . . . . . . . . . . . . . 29

2.4 Multiple slope regression fit to the two-ray model. . . . . . . . . . . . 31

2.5 Two-path model. . . . . . . . . . . . . . . . . . . 32

2.6 Reuse distance of a frequency division cellular wireless system. . . . . . 41

3.1 Cellular wireless system site coverage layout for a hexagonal tessellation. 47

3.2 Co-ordinates for hexagonal geometry site layout in a cellular wireless system 48

3.3 Cellular wireless system site coverage layout for a square tessellation. . . 49

3.4 Cellular wireless system site coverage layout for a equilateral triangle tessellation. . . . . . . . . . . . . . . . . 50

3.5 Illustration of how "shift parameters" are used for cellular system site layout. 51

3.6 Geometry of a four cluster size regular hexagonal cellular system site layout. 54 
3.7 Geometry of a seven cluster size regular hexagonal cellular system site layout. . . . . . . . . . . . . . . . . 55

3.8 Geometry of a two cluster size square cellular system site layout. . . . . . 56

3.9 Geometry of a four cluster size equilateral triangle cellular system site layout. . . . . . . . . . . . . . . . . . . 57

3.10 System propagation model for the uplink of a cellular wireless network. . 59

3.11 Two-slope received signal power, $P_{r}$ versus distance $R$. . . . . . . . . 62

3.12 Breakpoint distance, $g$ versus carrier frequency, $f_{c}$. . . . . . . . 63

3.13 Breakpoint distance, $g$ vrs. effective road height, $h . \ldots \ldots 6$

3.14 Proposed two tier interference system model. . . . . . . . . . . . . . . . 69

4.1 Geometry of desired user and interfering users in a two tier co-channel cells 77

4.2 Integrated simulation environment for information capacity analysis . . . 81

4.3 Flow chart for information capacity comparison simulations . . . . . . 82

4.4 Information capacity comparison between single and two tier co-channel interference model for carrier frequency, $f_{c}=900 \mathrm{MHz} . \quad \ldots \ldots 6$

4.5 Information capacity comparison between single and two tier co-channel interference model for carrier frequency, $f_{c}=2 \mathrm{GHz}$. . . . . . . . . 87

4.6 Information capacity comparison between single and two tier co-channel interference model for carrier frequency, $f_{c}=3.35 \mathrm{GHz} . \quad \ldots \ldots 88$

4.7 Information capacity comparison between single and two tier co-channel interference model for carrier frequency, $f_{c}=8.45 \mathrm{GHz} . \quad \ldots . . . . .889$

4.8 Information capacity comparison between single and two tier co-channel interference model for carrier frequency, $f_{c}=15.75 \mathrm{GHz}$. . . . . . . . . 90

4.9 Area spectral efficiency versus cell size radius for path loss exponent, $\alpha=2.93$

4.10 Area spectral efficiency versus cell size radius for path loss exponent, $\alpha$

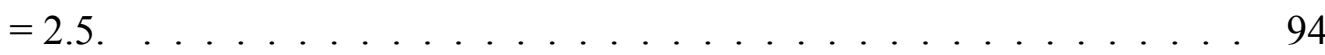

4.11 Area spectral efficiency versus cell size radius for path loss exponent, $\alpha$

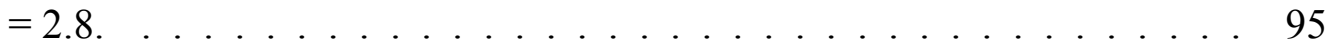

4.12 Area spectral efficiency versus cell size radius for path loss exponent, $\alpha=3.96$ 
4.13 Area spectral efficiency versus cell size radius for extra path loss exponent, $\rho=2$.

4.14 Area spectral efficiency versus cell size radius for extra path loss exponent, $\rho=3$.

4.15 Area spectral efficiency versus cell size radius for extra path loss exponent, $\rho=5$.

4.16 Area spectral efficiency versus cell size radius for extra path loss exponent, $\rho=8$.

4.17 Area spectral efficiency versus cell size radius for BS station antenna height $h_{b}=35 \mathrm{~m}$, and different carrier frequencies, $f_{c} \ldots \ldots 106$

4.18 Area spectral efficiency versus cell size radius for BS station antenna height $h_{b}=55 \mathrm{~m}$, and different carrier frequencies, $f_{c} \ldots \ldots 107$

4.19 Area spectral efficiency versus cell size radius for light vehicular traffic with effective road height and BS antenna height, $h=0.3 \mathrm{~m}$ and $h_{b}=15 \mathrm{~m} .109$

4.20 Area spectral efficiency versus cell size radius for heavy vehicular traffic with effective road height and BS antenna height $h=1.29 \mathrm{~m}$ and $h_{b}=15 \mathrm{~m} .110$

4.21 Area spectral efficiency versus cell size radius for light vehicular traffic with effective road height and BS antenna height, $h=0.3 \mathrm{~m}$ and $h_{b}=35 \mathrm{~m} .111$

4.22 Area spectral efficiency versus cell size radius for heavy vehicular traffic with effective road height and BS antenna height, $h=1.29 \mathrm{~m}$ and $h_{b}=35 \mathrm{~m} .112$

5.1 Two-slope received signal power, $P_{r}$ versus distance $R \ldots \ldots$. . . . 120

5.2 Two-slope received signal power, $P_{r}$ versus distance $R \ldots \ldots$. . . . 121

5.3 Two-slope received signal power, $P_{r}$ versus distance $R$. . . . . . . . . 122

5.4 Two-slope received signal power, $P_{r}$ versus distance $R \ldots \ldots$

5.5 Two-slope path loss model received signal power, $P_{r}$ versus distance, $R$. . 124

$5.6 \mathrm{n}$ tier co-channel interfering cells. . . . . . . . . . . . . 126

5.7 Multiple tier uplink co-channel interference on a desired BS. . . . . . . 127

5.8 Average area spectral efficiency versus number of co-channel interfering tiers. 
5.9 Average area spectral efficiency versus number of co-channel interfering

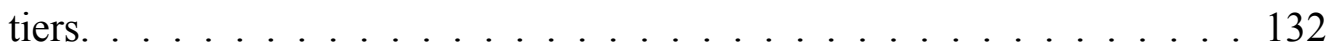

5.10 Average area spectral efficiency versus number of co-channel interfering

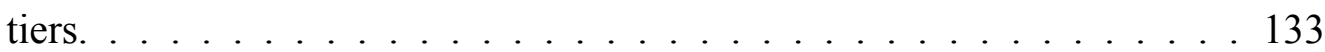

5.11 Geometry of desired user and interfering users in different co-channel tiers. 135

5.12 Flow chart for multiple tier interference information capacity simulations. 136

5.13 Information capacity comparison between single and multiple tier co-channel interference model for carrier frequency, $f_{c}=900 \mathrm{MHz}$. . . . . . . . 139

5.14 Information capacity comparison between single and multiple tier co-channel interference model for carrier frequency, $f_{c}=2 \mathrm{GHz}$. . . . . . . . . 140

5.15 Information capacity comparison between single and multiple tier co-channel interference model for carrier frequency, $f_{c}=3.35 \mathrm{GHz} . \quad \ldots . . . . .141$

5.16 Information capacity comparison between single and multiple tier co-channel interference model for carrier frequency, $f_{c}=8.45 \mathrm{GHz} . \quad \ldots . . . . .142$

5.17 Information capacity comparison between single and multiple tier co-channel interference model for carrier frequency, $f_{c}=15.75 \mathrm{GHz} . \ldots . . . .143$

5.18 Information capacity comparison between two and multiple tier co-channel interference model for carrier frequency, $f_{c}=900 \mathrm{MHz} . \quad \ldots . . . . .144$

5.19 Information capacity comparison between two and multiple tier co-channel interference model for carrier frequency, $f_{c}=2 \mathrm{GHz} . \ldots . . . . . .145$

5.20 Information capacity comparison between two and multiple tier co-channel interference model for carrier frequency, $f_{c}=3.35 \mathrm{GHz}$. . . . . . . . 146

5.21 Information capacity comparison between two and multiple tier co-channel interference model for carrier frequency, $f_{c}=8.45 \mathrm{GHz} . \quad \ldots . . . . .147$

5.22 Information capacity comparison between two and multiple tier co-channel interference model for carrier frequency, $f_{c}=15.75 \mathrm{GHz} . \ldots \ldots 148$

5.23 Area spectral efficiency versus cell radius for path loss exponent, $\alpha=2 . \quad .151$

5.24 Area spectral efficiency versus cell radius for path loss exponent, $\alpha=2.5 .152$

5.25 Area spectral efficiency versus cell radius for path loss exponent, $\alpha=2.8 .153$

5.26 Area spectral efficiency versus cell radius for path loss exponent, $\alpha=3$. . 154

5.27 Area spectral efficiency versus cell radius for path loss exponent, $\alpha=2 . \quad$. 155 
5.28 Area spectral efficiency versus cell radius for path loss exponent, $\alpha=2.5$. 156

5.29 Area spectral efficiency versus cell radius for path loss exponent, $\alpha=2.8 .157$

5.30 Area spectral efficiency versus cell radius for path loss exponent, $\alpha=3$. . 158

5.31 Area spectral efficiency versus cell radius for extra path loss exponent, $\rho$

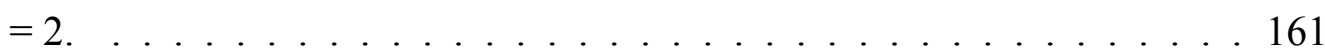

5.32 Area spectral efficiency versus cell radius for extra path loss exponent, $\rho$ $=3$.

5.33 Area spectral efficiency versus cell radius for extra path loss exponent, $\rho$ $=5$.

5.34 Area spectral efficiency versus cell radius for extra path loss exponent, $\rho$ $=8$.

5.35 Area spectral efficiency versus cell radius for BS antenna height $h_{b}=35 \mathrm{~m} .166$

5.36 Area spectral efficiency versus cell radius for BS antenna height $h_{b}=55 \mathrm{~m} .167$

5.37 Area spectral efficiency versus cell radius for light vehicular traffic with BS antenna height, $h_{b}=15 \mathrm{~m}$ and effective road height, $h=0.3 \mathrm{~m} . \ldots 169$

5.38 Area spectral efficiency versus cell radius for heavy vehicular traffic with BS antenna height, $h_{b}=15 \mathrm{~m}$ and effective road height, $h=1.29 \mathrm{~m} . \quad \ldots 170$

5.39 Area spectral efficiency versus cell radius for light vehicular traffic with BS antenna height, $h_{b}=35 \mathrm{~m}$ and effective road height, $h=0.3 \mathrm{~m} . \ldots 171$

5.40 Area spectral efficiency versus cell radius for heavy vehicular traffic with BS antenna height, $h_{b}=35 \mathrm{~m}$ and effective road height, $h=1.29 \mathrm{~m}$

6.1 Infrastructure cost model. . . . . . . . . . . . . . . . . . . . . . . 180

6.2 Cellular system unit cost per $\mathrm{km}^{2}$ versus cell radius R. . . . . . . . . . . 182

6.3 Integrated simulation environment for information capacity/cost. . . . . . 183

6.4 Flow chart for information capacity/cost simulations. . . . . . . . . . . . 184

6.5 Information capacity/cost comparison between single and multiple tier interference model, carrier frequency, $f_{c}=900 \mathrm{MHz}$. . . . . . . . . . . 187

6.6 Information capacity/cost comparison between single and multiple tier interference model, carrier frequency, $f_{c}=2 \mathrm{GHz} \ldots \ldots \ldots 188$ 
6.7 Information capacity/cost comparison between single and multiple tier interference model, carrier frequency, $f_{c}=3.35 \mathrm{GHz}$. . . . . . . . . 189

6.8 Information capacity/cost comparison between single and multiple tier interference model, carrier frequency, $f_{c}=8.45 \mathrm{GHz}$. . . . . . . . . 190

6.9 Information capacity/cost comparison between single and multiple tier interference model, carrier frequency, $f_{c}=15.75 \mathrm{GHz}$. . . . . . . . . . 191

6.10 Cost per information capacity comparison between single and multiple tier interference model, for different cell radius $R$. . . . . . . . . . . . 193

6.11 Heterogeneous Network. . . . . . . . . . . . . . . . . . . . . . . 197

6.12 A cellular system site layout with fractional frequency reuse . . . . . . 198

6.13 Area spectral efficiency versus cell radius for path loss exponent, $\alpha=2$ and reuse factor $1 / 3 \ldots \ldots 200$

6.14 Area spectral efficiency versus cell radius for path loss exponent, $\alpha=2$ and reuse factor one. . . . . . . . . . . . . . . 201 


\section{LIST OF TABLES}

3.1 Calculated Breakpoint Distance for Different Carrier Frequencies _ . . . 61

3.2 Breakpoint Distance for Microwave Band (Light Vehicular Traffic) . . . 64

3.3 Breakpoint Distance For Microwave Band (Heavy Vehicular Traffic) . . 65

4.1 Simulation Parameters for Information Capacity Comparison . . . . . . . 84

4.2 Percentage Decrease in Area Spectral Efficiency between single and two tier Interference Model for Different Cell Size Radius $R$. . . . . . . . . . 91

4.3 Percentage Decrease in Area Spectral Efficiency between single and two tier Interference Model for Different Basic Path loss Exponent and Cell Size Radius, $R=100 \mathrm{~m}$. . . . . . . . . . . . . . . . . . . 97

4.4 Percentage Decrease in Area Spectral Efficiency between single and two tier Interference Model for Different Basic Path loss Exponent and Cell Size Radius, $R=300$ m. . . . . . . . . . . . . . . . . . . 98

4.5 Percentage Decrease in Area Spectral Efficiency between single and two tier Interference Model for Different Extra Path loss Exponent and Cell Size Radius, $R=100$ m. . . . . . . . . . . . . . . . . . . 104

4.6 Percentage Decrease in Area Spectral Efficiency between single and two tier Interference Model for Different Extra Path loss Exponent and Cell Size Radius, $R=300 \mathrm{~m}$.

6.1 Basic cost drivers for radio access network . . . . . . . . . . . . . . . . 181 


\section{LIST OF PhD CANDIDATE'S \\ PUBLICATIONS RELATED TO PhD THESIS}

\section{Journal Papers:}

1. K. A. Anang, P. B. Rapajic, T. I. Eneh and R. Wu. "Minimum cell size for information capacity increase in a shadowed environment of cellular system operating at carrier frequency greater than $2 \mathrm{GHz}$," Eurasip Journal wireless communications and networking (to be submitted).

2. K. A. Anang, P. B. Rapajic, T. I. Eneh and R. Wu "Minimum cell size for information capacity increase in cellular wireless network,". Wireless Communications and Mobile Computing. (Accepted)

3. K. A. Anang, P. B. Rapajic, R. Wu, L. Bello and T. I. Eneh "Cellular system information capacity change at higher carrier frequencies due to propagation loss and system parameters," Progress In Electromagnetics Research B. ISSN: 1937-6472, vol. 44, pp. 191-221, Oct. 2012.

4. K. A. Anang, P. B. Rapajic, L. Bello and R. Wu "Sensitivity of cellular wireless network performance to system \& propagation parameters at carrier frequencies greater than 2 GHz," Progress In Electromagnetics Research B, ISSN: 1937-6472, vol. 40, pp. 31-54, Apr. 2012.

5. L. Bello, P. Bakalis, P. B. Rapajic, K. A. Anang, and T. I. Eneh. "Adaptive Receiver Power Routing Protocol for Mobile Ad Hoc Wireless Network," Journal of Com- 
munications (JCM): Special Issue: Advances in wireless comm. and networks. vol. 6, no. 7, pp. 571-580, Oct. 2011.

6. T. I. Eneh, G. Oletu, P. B. Rapajic, K. A. Anang, and L. Bello. "Adaptive multiuser receivers scheme for MIMO OFDM over iterative-equalization for singlecarrier transmission," Journal of emerging trends in engineering and applied sciences (JETEAS). ISSN: 2141-7016, vol. 2, no. 2, pp. 282-288, April, 20011.

\section{Conference Papers:}

1. K. A. Anang, L. Bello, P. B. Rapajic, T. I. Eneh and P. Bakalis. "Impact of propagation loss parameters on dynamic source routing protocol at higher microwave carrier frequencies" 14th IEEE International Conference on communication Technology (IEEE-ICCT 2012). Nov. 2012, Chengdu, China. (Accepted)

2. K. A. Anang, P. B. Rapajic, R. Wu. "Impact of sectorization on the minimum cell size for information capacity increase in cellular wireless network" IX International Symposium Industrial Electronics (INDEL 2012). Banja Luka, Bosnia and Herzegovina, pp. 220 - 225, Nov. 2012.

3. K. A. Anang, P. B. Rapajic, R. Wu, G. Oletu and L. Bello. "Minimum cell size for information capacity increase in a shadowed environment of land mobile cellular system" 7th IEEE International Conference on Broadband and Wireless Computing, Communication and Applications (BWCCA 2012). Victoria, Canada, pp. 122 126, Nov. 2012.

4. K. A. Anang, T. I. Eneh, L. Bello and D. L. Larkai" The influence of vehicular traffic on cellular wireless system performance at microwave carrier frequencies" 4th IEEE International Conference on Adaptive Science and Technology (4th IEEE ICAST 2012). Kumasi, Ghana, pp. 17 - 22, Oct. 2012.

5. K. A. Anang, P. B. Rapajic, T. I. Eneh and B. Lawal. "Impact of propagation loss parameters on cellular wireless network operating at frequencies greater than $2 \mathrm{GHz}$ " 8th IEEE International Wireless Communications and Mobile Computing Conference (IEEE- IWCMC 2012). Limassol, Cyprus, pp. 1169 - 1175, Aug. 2012. 
6. L. Bello, K. A. Anang, P. Bakalis, P. B. Rapajic and T. I. Eneh "Sensitivity of DSR protocol performance to propagation Loss models at higher microwave frequencies" 14th IEEE International Conference on Computer Modelling Simulation (14th IEEE UKsim 2012), Cambridge, UK, pp. 561 - 565, Mar. 2012.

7. K. A. Anang, P. B. Rapajic, T. I. Eneh, L. Bello and G. Oletu. "Impact of vehicular traffic on information capacity of cellular wireless network at carrier frequencies greater than $3 \mathrm{GHz}$ " 5th IEEE European Modelling Symposium on Mathematical modelling and Computer Simulation (5th IEEE EMS 2011), Madrid, Spain, pp. 430 - 434, Nov. 2011.

8. P. Bakalis, L. Bello, O. Jagun, K. A. Anang, T. I. Eneh and A. Muhammad" Performance evaluation of constant bit rate and variable bit rate traffic models on vehicular Ad hoc network using dynamic source routing protocol" 3rd IEEE International conference on Adaptive Science and Technology (3rd IEEE ICAST 2011), Abuja, Nigeria, pp. 187 - 191, Nov. 2011.

9. T. I. Eneh, P. B. Rapajic, K. A. Anang, P. Bakalis and L. Bello" Information capacity of Adaptive MIOM OFDM" 3rd IEEE International conference on Adaptive Science and Technology (3rd IEEE ICAST 2011), Abuja, Nigeria, pp. 157 - 164, Nov. 2011.

10. G. Oletu, P. B. Rapajic, T. I. Eneh, and K. A. Anang. "Enhanced equalization for mobile communication systems" 5th IEEE European Modelling Symposium on Mathematical modelling and Computer Simulation (5th IEEE EMS 2011), Madrid, Spain, pp. 446 - 450, Nov. 2011.

11. K. A. Anang, L. Bello, T. I. Eneh, P. Bakalis and P. B. Rapajic. "The performance of dynamic source routing protocol to path loss models at carrier frequencies above 2 GHz," 13th IEEE International Conference on communication Technology (IEEEICCT 2011), Jinan, China, pp. 151 - 155, Sept. 2011.

12. K. A. Anang, P. B. Rapajic, T. I. Eneh and B. Lawal. "Sensitivity of information capacity of land mobile cellular system to propagation loss parameters at higher microwave frequencies". In Proc. of 7th IEEE International Wireless Communica- 
tions and Mobile Computing Conference (IEEE - IWCMC 2011). Istanbul, Turkey, pp. 630-635, July 2011.

13. T. I. Eneh, G. Oletu, P. B. Rapajic, K. A. Anang, and B. Lawal. "Adaptive MMSE multiuser detection for MIMO OFDM over turbo-equalization for single-carrier transmission wireless Channel". In Proc. of 7th IEEE International Wireless Communications and Mobile Computing Conference (IEEE - IWCMC 2011). Istanbul, Turkey, pp. 1408-1412, July 2011.

14. G. Oletu, P. B. Rapajic, T. I. Eneh and K. A. Anang. "Iterative equalization enhanced high data rate in wireless communication networks". 9th International Symposium on Modelling and Optimization in Mobile, Ad Hoc, and Wireless Networks (IEEEWiOpt'11). May 9th - 13th, 2011, New Jersey, USA.

15. K. A. Anang, P. B. Rapajic, T. I. Eneh and Y. Nijsure. "Minimum cell size for information capacity increase in cellular wireless network". In Proc. of 73rd IEEE Vehicular Technology Conference - spring (IEEE VTC- 2011, spring). Budapest, Hungary, pp. 305-311, May 2011.

16. T. I. Eneh, P. B. Rapajic, K. A. Anang and B. Lawal. "Information capacity comparisons over Gaussian communication channels with ISI when used with single carrier transmission with MMSE - DFE receiver or multicarrier OFDM receivers”. In Proc. of 73rd IEEE Vehicular Technology Conference - spring (IEEE VTC- 2011, spring). Budapest, Hungary, pp. 56-62, May 2011.

17. T. I. Eneh, G. Oletu, P. B. Rapajic, K. A. Anang, and B. Lawal. "Adaptive vector precoding for Multiuser MIMO OFDM". International conference on ICT for Africa (ICT4Africa 2011). Covenant University, Canaan Land, Nigeria.

18. B. Lawal, P. Bakalis, S. Manam, T. Eneh and K. A. Anang. "Power control and performance comparison of AODV and DSR," in Proc. of Computer modelling and simulation (UKSIM), 2011 UKSIM , IEEE 13th International Conference on Computer Modelling and Simulation (IEEE- UKSIM2011), Cambridge, UK, pp. 457-460, April 2011. 
19. K. A. Anang, P. B. Rapajic, T. I. Eneh and G. Oletu. "Sensitivity of information capacity of land mobile cellular system to the base station antenna height at higher microwave frequencies," in Proc. of 3rd International Conference on Computer Research and Development (IEEE- ICCRD 2011). Shanghai, China, pp. 167-172, Mar. 2011.

20. T. I. Eneh, P. B. Rapajic and K. A. Anang and B. Lawal. "Adaptive MMSE Multiuser Detection in MIMO OFDM Wireless Communication System” 3rd International Conference on Engineering Research and Development (ICERD2010), Benin City, Nigeria.

21. T. I. Eneh, P. B. Rapajic, K. A. Anang, B. Lawal and A. Qureshi,"Information capacity limits of the communication system with adaptive MMSE receivers," in Proc. of 1st International Conference on Future Information Technology (ICFIT 2010), Changsha, China, pp. V1-67 - V1-71, Dec. 2010.

22. T. I. Eneh, P. B. Rapajic and $K$. A. Anang. "Adaptive MMSE multiuser receivers in MIMO OFDM wireless communication system." in Proc. of 12th IEEE International Conference on communication Technology (IEEE-ICCT2010), Nanjing, China, pp. 934-937, Nov. 2010. 
Chapter 1

\section{Introduction}




\subsection{Research Aims of the Thesis}

This thesis provides a formula for calculating the number of co-channel interfering cells in subsequent tiers of a wireless communication system site layout. It addresses a long standing question regarding why at higher carrier frequencies and smaller cell size radii, there is a problem of increase co-channel interference $[1,2]$ ? which leads to a reduction in the uplink information capacity of the cellular system [3]. The thesis addresses the aforementioned question by showing that at higher carrier frequencies and smaller cell size radii, other tier co-channel cells becomes active, thus increasing co-channel interference. In cellular wireless communication systems ranges of maximum and minimum cells size radius have being reported [4-6]. It has also been suggested that there may be a limit to cell size radius reduction [7]. However, there are no explanations or proof for them. In this thesis we find a theoretical limit to cell size radius reduction in cellular wireless systems which is founded on the uplink information capacity and infrastructure cost of the cellular system.

The remainder of the Chapter is organized as follows. A general background for the study is first presented in Chapter 1.2, including an exposé of wireless communication and a brief history of the development of wireless communications. Chapter 1.3, focus on the future and future development of wireless communications. A discussion on the possible limitations which will hamper the implementation of the wireless future development is presented in Chapter 1.4. Chapter 1.5, explains motivations that led to this work at a high level and states the thesis contribution to the body of knowledge. Finally, Chapter 1.6, provides an outline of the thesis.

\subsection{Wireless Communication}

The transfer of information over a distance without the use of electrical conductors or wires (Wireless Communications), paved the way in breaking the location barrier in telecommunications. To break this barrier, information has to be transmitted through an antenna which converts a radio frequency (RF) signal into an electromagnetic wave. This electromagnetic wave is intercepted by a receiving antenna which converts it back to a RF 


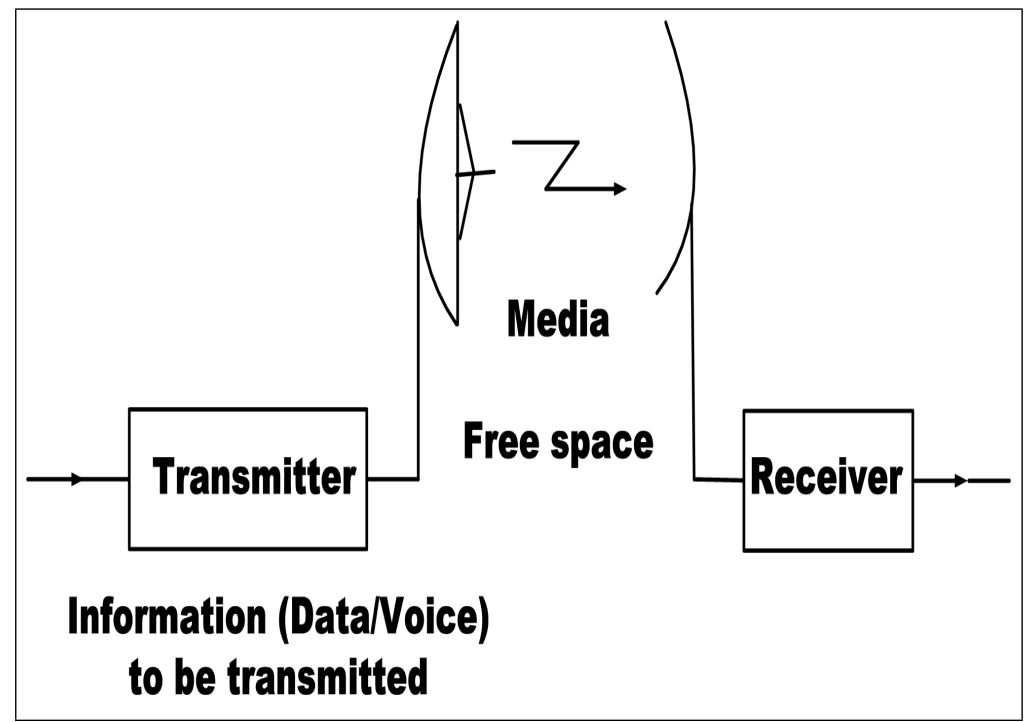

Figure 1.1: A basic wireless communication system showing a transmitter and a receiver.

signal. The original information received by the antenna is then demodulated back to its original form. The received RF signal is ideally, supposed to be the same as originally generated by the transmitter. However, the received RF signal is usually degraded, due to environmental conditions or interference from other electromagnetic sources, thus making interference one of the technical challenges in advancing the future of wireless communications. It is therefore, important to study the effect of interference on the information capacity of wireless communication systems operating at higher carrier frequencies and smaller cell size radius. This study is one of the goals of this thesis. For the purpose of this thesis a wireless communication system is shown in Figure 1.1. A brief description of how an electromagnetic wave is generated, and how interference affects reception of the best possible RF signal from a source is given in the following section.

\subsubsection{Electromagnetic Waves Propagation and Interference}

To generate an electromagnetic wave, an electrical signal, which continually varies in power level and changes in polarity, is applied to an antenna. The continuous variation in power level and change in polarity causes the energy in the electrical signal to be converted into electromagnetic waves. The electromagnetic wave then radiate away from the antenna and propagate into free space. Free space is basically the transmission medium for 
electromagnetic waves. Electromagnetic waves are characterized by their radio frequency power (RF power) expressed in $[\mathrm{W}]$ or $[\mathrm{dBm}]$. The $[\mathrm{dBm}]$, is the power ratio in decibels of the measured power with reference to $1 \mathrm{~mW}$. The frequency or cycles per second is usually expressed in Hertz (Hz).

In general, interference occurs when correlated or coherent radiated electromagnetic waves superimpose on each other to form a resultant wave of greater or lower amplitude. In wireless communication this interference alters, modifies or disrupts a signal as it travels along a channel between a source and a receiver. Interferences, such as radio frequency interference (RFI), co-channel interference (CCI) and adjacent channel interference (ACI) affect the information capacity performance of a wireless network. This thesis focuses on co-channel interference in cellular wireless networks. The next section presents a brief historical development of wireless communication and the concept of cochannel interference.

\subsubsection{History of Cellular Wireless Communications and Influence of Co-channel Interference}

The evolution of wireless communications dates back to 1802. James Bowman Lindsay gave a classroom demonstration of an Ultra High Frequency (UHF) wireless telegraphy operation to his students in Dundee. Followed by James Clerk Maxwell using the concept of magnetic induction introduced by Michael Faraday to establish equations. These equations formed the basis for explaining electromagnetic wave propagation. However, Maxwell's equations did not show how electromagnetic waves could be generated and detected practically.

Heinrich Hertz, in 1886 - 1887, demonstrated how to generate and detect electromagnetic waves, using spark generators, dipole transmitting, and loop receiving antennas. However, the distance covered was just a few metres [8,9]. In 1890, Edouard Branly developed the coherer, a more sensitive detector, which increased the distance at which generated electromagnetic waves can be detected. Later, Sir Oliver Lodge, improved upon the coherer developed by Edouard Branly.

Nicola Tesla in 1891, demonstrate the transmission of electrical energy in free 
space to the American Institute of Electrical Engineers. This latter earned Nicola the title "Father of the wireless". In 1896, Guglielmo Marconi demonstrated wireless telegraph to the English telegraph office. The year 1897, saw "The Birth of Radio", when "Marconi Station" established on Needless Island communicated with English coast. In 1897 too, a wireless transmission, recorded by the British Post Office, reached $14 \mathrm{~km}$ in the Bristol Channel [10]. In 1899, a signal crossed the English Channel and in 1901, Marconi successfully sent wireless message across the Atlantic Ocean, from Poldhu in England to Signal Hill in Newfoundland [11], and 1902, was when the first bidirectional wireless communication across the Atlantic took place. During this period of evolution, interference was not much of a problem as the radio spectrum was not crowded.

The first voice over wireless transmission occurred in 1914. However, the public had to wait until 1946, when AT \& T inaugurated the first public mobile telephone service in St. Louis. AT \& T connected mobile users to the Public Switched Telephone Network (PSTN). After the inauguration, the number of mobile phone service providers and users begin to increase, the block of frequency spectrum allocated to service providers was limited. As the number of mobile users begin to increase, there was a need to meet the demand of a larger subscriber capacity. Meeting this demand led to the evolution of the cellular concept.

A cellular system operates within a limited block of frequency spectrum to meet an objective of providing an increase in information capacity. The cellular concept entails the reuse of radio channels on the same carrier frequency to cover different areas. These areas are separated from each other by sufficient distance to avoid/minimise co-channel interference. As the number of mobile users continues to grow, service providers were forced to put up more cellular towers. The putting up of more cellular towers increased the problem of interference in wireless communications, which negatively affects the increase in information capacity of the cellular wireless system.

The continuous increase of mobile users led to the deployment of the first generation of mobile systems (1G) in the 1980's. Like what happened to wireline capacity in the 1990's, the demand for new wireless information capacity kept growing at a fast pace. However, the conventional resources that have been used to add capacity to wireless systems are radio bandwidth and transmitter power. These resources are limited, hence they 
are not growing at the rates that can support the increased demands for wireless information capacity. This has driven most research in wireless communications towards finding alternative ways of increasing the wireless information capacity. Some of this research included the development of new wireless information capacity through the use of greater intelligence in wireless networks $[12,13]$. The deployment of higher microwave carrier frequencies and smaller cell size radius cellular wireless communication systems [14-16]. These researches have led to subsequent generation of wireless communication systems such as $2 \mathrm{G}, 3 \mathrm{G}$ and $4 \mathrm{G}[17,18]$.

However, at higher frequencies and smaller cell size radius interference becomes a problem in cellular system [1]. There is no limit to how small a cell size must be to achieve information capacity increase. Likewise, existing interference models were founded on lower carrier frequencies and lager cell size radius, therefore the direction of this thesis

\subsection{Possible Future Development Direction of Cellular Wire- less Communications}

The introduction of cellular wireless and cordless telephone systems in the early 1980s caused wireless communication systems and services to undergo a remarkable development and growth [19]. The need to operate and increase the information capacity of wireless networks indefinitely within an allocation of hundreds of frequency channels has been the primary driving force behind the evolution of the cellular concept. The future development of wireless communication providing faster, high-speed, high-quality and real time information exchange between two portable devices located anywhere in the world is now the communications frontier of the 21 st century [20].

The popularity of wireless systems; such as cordless phones, cellular telephones, radio paging, satellite networks and sensor networks wireless local area networks (WLANs) demonstrates a great demand for such services and all the components of the future development of wireless. Likewise, wireless applications; such as voice, internet access, distributed control, ad-hoc wireless networks, wide area wireless data systems and multimedia. Figure 1.2, illustrates the possible future development of wireless communication 


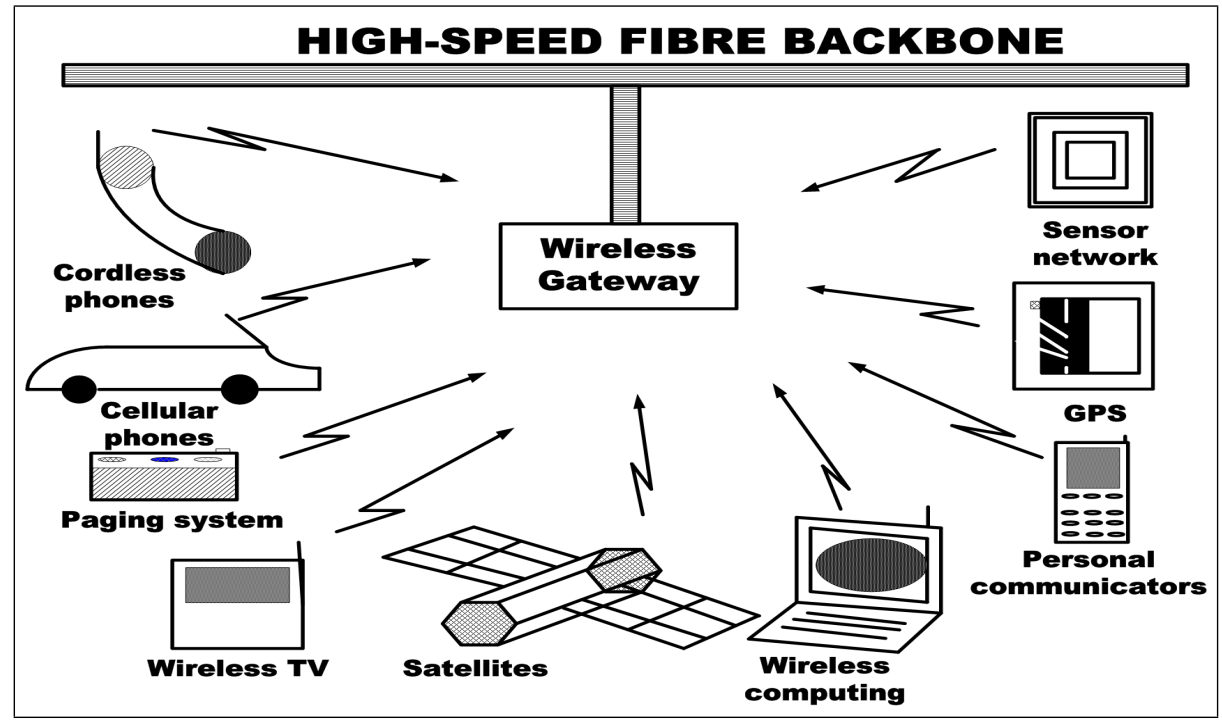

Figure 1.2: The future development for wireless communication systems [20].

systems. Internetworking is achieved via a wireless gateway and a high-speed fibre backbone, which is then connected to existing cellular wireless systems.

During the last decade it has emerged from wireless communication research and development activities that the wireless future is based on three trends: a broader range of wireless communication products, high data-rate wireless communication systems and higher user density [21]. It must be noted that to attain high data-rates in wireless communication, interference must be minimal. To achieve this wireless future development, there is a need to utilize the limited radio spectrum efficiently. In the quest to utilize the radio spectrum efficiently cell size radii are to be smaller and frequency of operation are to be higher $[2,7]$.

However, at higher carrier frequencies and smaller cell size radius, there is a problem of co-channel interference. This interference problem motivates and determines the direction of the research in the thesis. The next section gives an overview of the technical issues involved in the implementation of the future development of wireless communication. A number of these issues will be examined in the thesis chapters. New methods are proposed to evaluate, and approach them, and compare these new methods with other existing techniques that are currently being implemented or suggested in literatures. 
Technical Issues Related to the Future of Wireless Communication

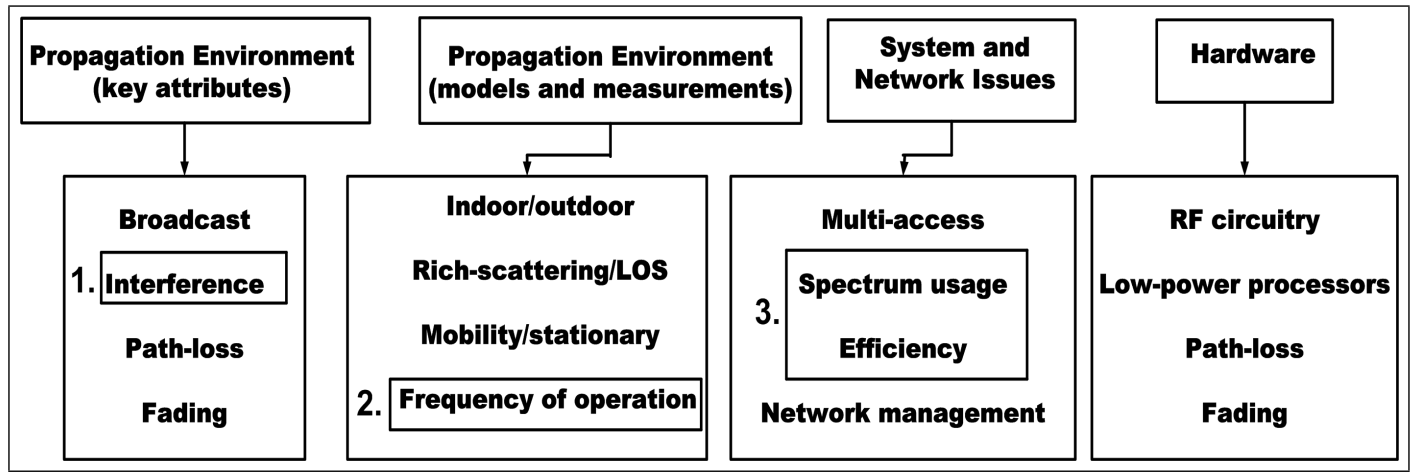

Figure 1.3: Some technical challenges to be addressed in implementing the future wireless communication systems.

1. Interference - related to this thesis

2. Frequency of operation - related to this thesis

3. Spectrum usage and efficiency - related to this thesis

\subsection{Technical Issues Related to the Future of Wireless Communication}

Technical issues related to wireless communication future cover limitations which arise from the technology chosen for the wireless system implementation. These technical challenges need to be considered in the modelling and design of wireless communication systems to enable its implementation, notably:

- Spectrum limitations in wireless communications

- Energy limitations of personal mobile device

- Interference in wireless communications

- Multipath signal propagation in wireless communication

- User mobility within a cell

This thesis will focus more on interference modelling. A table representation of some of the technical challenges which need to be addressed is given in Figure 1.3. The figure also shows the three technical challenges which are related to this thesis. It is necessary to 
understand the fundamental technical issues for determining the feasibility of a given wireless technology with respect to the absolute physical communication limits. The wireless communication channel is susceptible to noise, interference, multipath signal propagation and user's movement, which causes the wireless communication channel to change over time in an unpredictable manner [22]. Wireless mobile multimedia communication has captured the attention of the media and the imagination of the public. It is because wireless communication allows connectivity between users without sacrificing mobility. However, it is fundamentally limited by the information capacity of the wireless communications channel. It must be noted that interference limits the information capacity more than any other single effect [23]. Modelling interference accurately is therefore important for the successful implementation of the wireless future development, which is part of the focus of this thesis. The wireless communication network information capacity is defined as the highest data rate (bit) at which information can be sent over the wireless communication network with a negligible probability of error. A wireless communication channel is illustrated in Figure 1.4, showing noise, interference and multipath signal propagation.

Multi-path signal propagation occurs, when a radio signal transmitted from a fixed source to a mobile station (MS) experiences variation in amplitude and phase. The variation, arises when the transmitted signal is reflected, diffracted or scattered by objects. These reflected, diffracted or scattered signals create additional copies of the transmitted signal. The additional copies of the signal can be attenuated in power, delayed in time, and shifted in carrier phase and/or carrier frequency from the line-of-sight (LOS) signal path. The LOS signal path is the straight line path between the transmitter and receiver. Multipath signal propagation causes the received signal amplitude to vary. The time delay of each path causes intersymbol interference.

Power and size of wireless mobile multimedia communication and computing devices present another limitation unlike vehicular wireless communication devices which have only some power or size limitations. For personal wireless communication devices they are meant to be carried in wallet or pocket. These devices need to be small and lightweight, which translates to requirement of low power, because small batteries must be used. The information capacity of the wireless communication channel is determined 


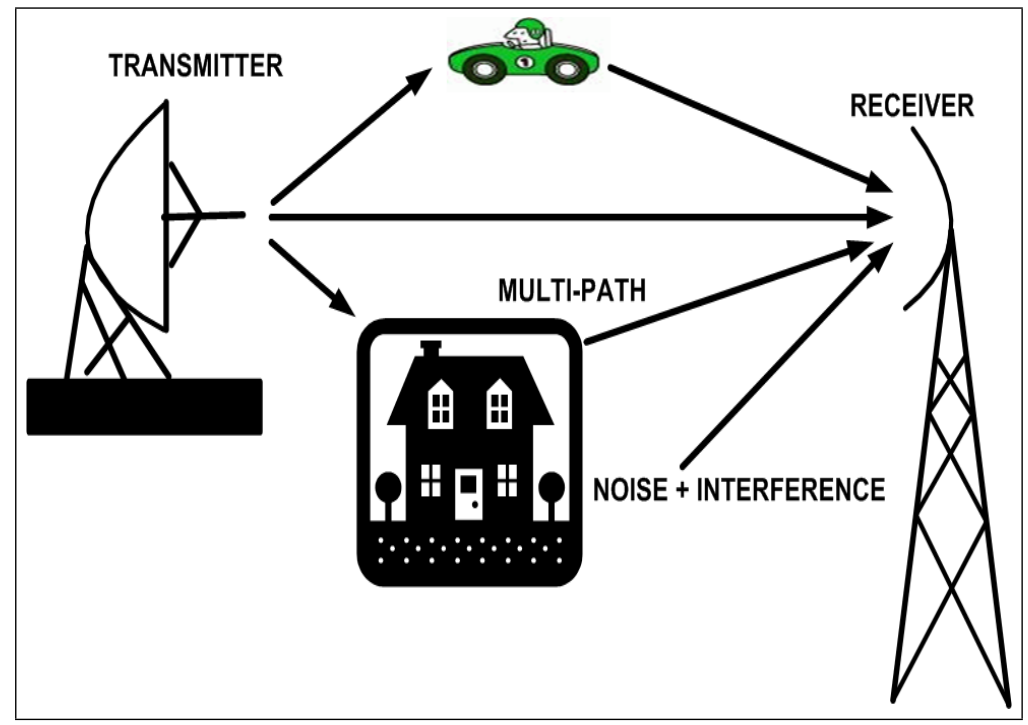

Figure 1.4: A wireless communication channel showing a direct signal path, and multiple signal path propagation from a transmitter to a receiver.

by the channel information transmission resources, which includes: time, bandwidth and power (signal-to-noise ratio) [24].

Transmitting more power to increase the channel capacity or data rate of the wireless communication system is costly, because of the logarithmic relationship between the channel capacity of the wireless communication link and the signal-to-noise ratio (SNR) at the receiver. This logarithmic relationship was first developed by Shannon in his landmark 1948 paper "mathematical theory of communications" [25]. By Shannon's result, the channel capacity is given by:

$$
\mathcal{C}=B \log \left(1+\frac{S}{N}\right)
$$

where $B$ is the signal bandwidth, $S$ the signal power and $N$ the noise. The ratio $S / N$ is the signal-to-noise ratio (SNR). Thus, asymptotically error-free communication at information rates below $\log (1+\mathrm{SNR})$ are possible, while transmission at any rate larger than $\log (1+\mathrm{SNR})$ is guaranteed to have errors.

Another effective method for increasing data rate in wireless systems is to increase the signal bandwidth, in addition to the transmitted power. However, the radio spectrum available for wireless communication systems is expensive and limited [26]. It is therefore, regulated by government authorities and wireless communication standardizing 
Technical Issues Related to the Future of Wireless Communication

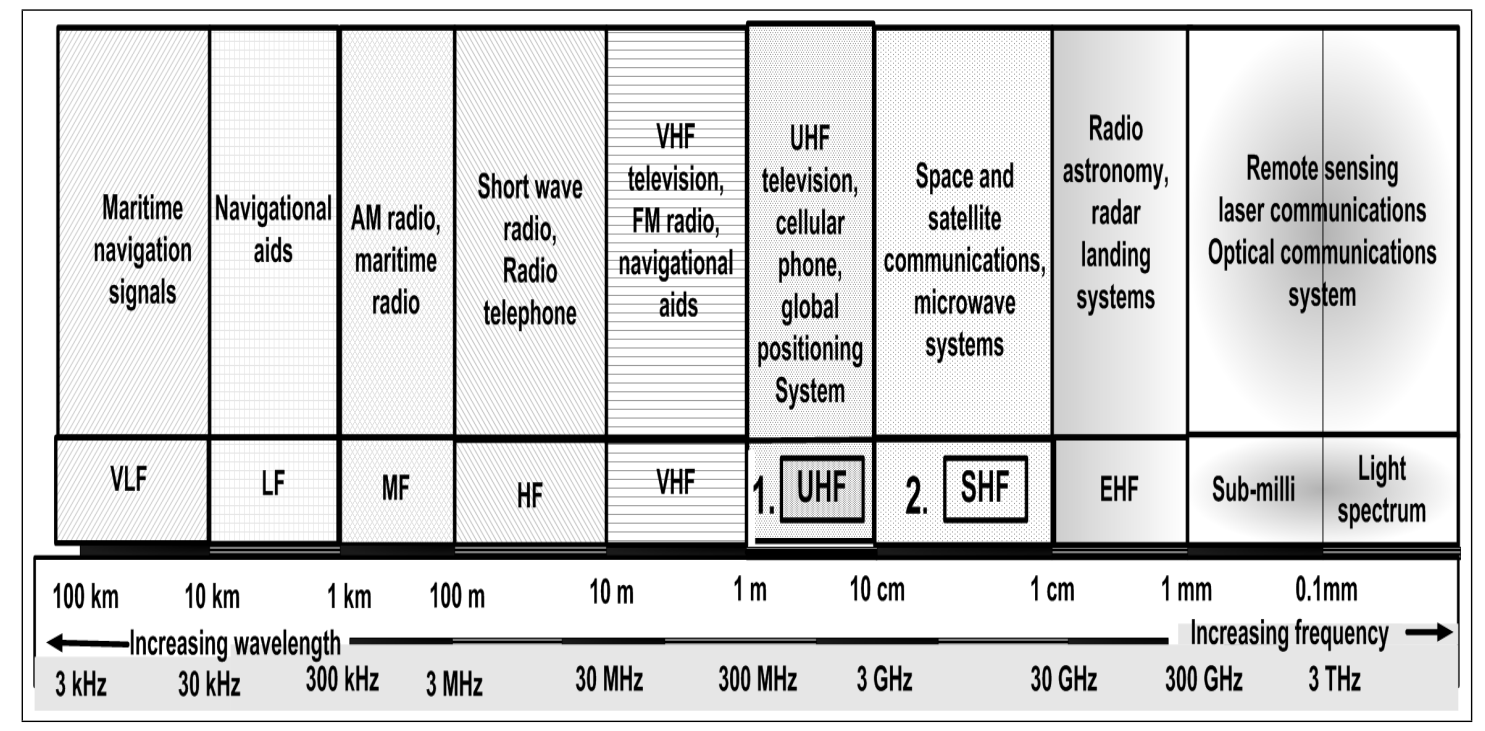

Figure 1.5: Wireless communications radio spectrum allocation and description of the frequency bands of interest for the purpose of wireless communications.

1. Conventional frequency for wireless systems

2. Emerging and expected frequency for future wireless systems

organizations [27]. The allocation and application of the various spectrum band is illustrated in Figure 1.5. The description of the bandwidth and wavelength are specified in Hertz and meters respectively. The figure also shows the UHF and SHF band of interest for the purpose of wireless communications. The UHF band is used for conventional wireless system and the SHF band is expected to be used for possible future wireless development to achieve higher information capacity.

As the radio spectrum is limited, efficient utilization of the radio spectrum is important in the design of wireless communication system. With the recent increase in spectrum allocation for wireless applications. The radio spectrum has been stretched to its capacity to accommodate the various wireless services (that is, it has become congested). In order to increase the information capacity of cellular systems, and the associated congestion of radio spectrum especially the UHF band, where propagation conditions for conventional cellular system are favorable, carrier frequencies for emerging cellular system are excepted to be shifted to the SHF band. However, in the SHF band (higher carrier frequency) signal propagation conditions are different from that of the UHF band. At the 
SHF band signal propagation are different from that of the UHF band, because (i) at SHF band signals travels shorter distances, as compared to those of the UHF band, therefore cells size radius needs to be smaller (ii) UHF radio wave propagates mainly by LOS, whilst SHF radio wave propagate entirely by LOS, groundwave and skywave. SHF radio wave propagation is more vulnerable to the non-line-of-sight condition [28]. Interference modelling for the SHF band are therefore excepted to be different from those of the conventional UHF band. Therefore, interference modelling for the SHF band is one of the focus for this thesis.

\subsubsection{Spectral Efficiency}

The spectral efficiency of a wireless communication system is defined as the information rate that can be transmitted over a given bandwidth in a specific wireless communication system and it is measured in bits $/ \mathrm{s} / \mathrm{Hz}$. The system information capacity of a wireless communication system is directly related to the spectral efficiency, hence it is an important parameter to be considered in the design of wireless communication system [26, 29, 30]. Efficient utilization of the congested radio spectrum is partly achieved by the cellular networks [4]. Other techniques to increase spectral efficiency include methods such as combination of bandwidth efficient coding/modulation techniques at the communication link level and the use of sophisticated channel allocation schemes that minimize the overall carried traffic at the network or systems levels [31]. Techniques to utilize the radio spectrum efficiently and effectively share it have been a major design concern for emerging wireless communication systems. Thus, we have used the spectral efficiency for the information capacity performance analysis in this thesis.

\subsubsection{Interference in Cellular Wireless Systems}

Interference is the major limiting factor that affects the information capacity of cellular wireless systems, it has been recognized as a main obstacle for increasing information capacity $[3,20,32,33]$. Because of this, the information capacity performance of wireless communication systems operating under the effect of interference needs to be studied extensively [34-36]. The two major types of system-generated interference are: co-channel 


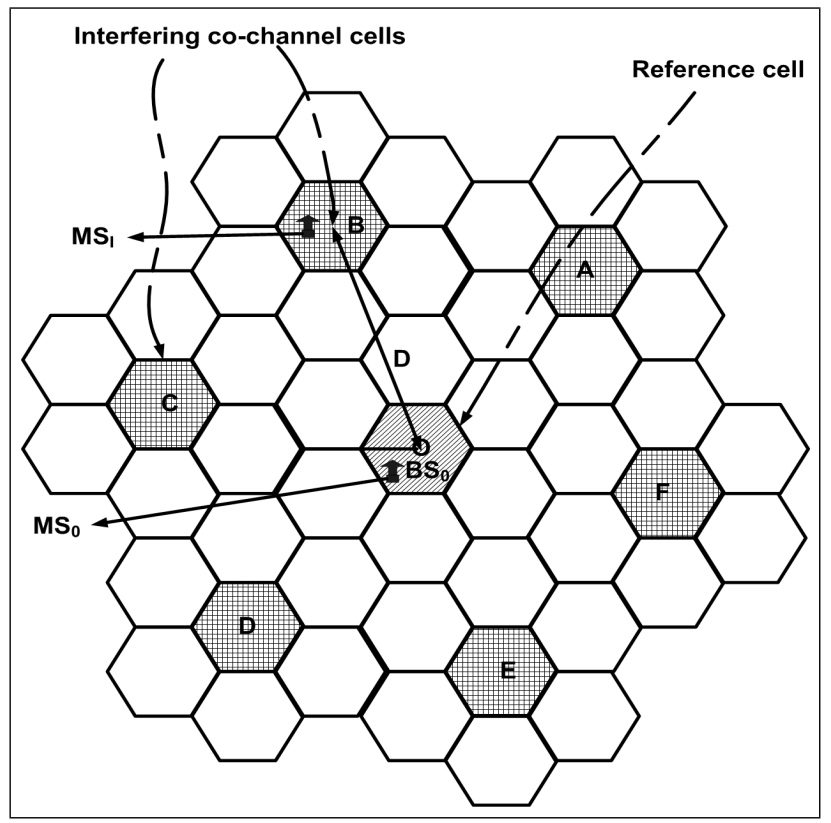

Figure 1.6: Conventional cellular wireless system showing a reference cell and first tier co-channel cells.

1. D : reuse distance for using the same carrier frequency

2. $\mathrm{MS}_{0}$ : desired mobile station

3. $\mathrm{MS}_{I}$ : interfering mobile station

4. $\mathrm{BS}_{0}:$ reference cell

5. A,B,C,D,E,F : first tier co-channel interfering cell

and adjacent channel interference. Co-channel interference (CCI) does occur in cellular wireless communication systems, for example, when a mobile station simultaneously receives signals from base stations (BSs'). In this case, one co-channel forward link is the desired signal, and the other co-channel signals are received as interference and compose the total co-channel interference at the receiver. Adjacent channel interference is caused by power from a signal in an adjacent channel. Adjacent channel interference may be caused by inadequate filtering, improper tuning or poor frequency control. Figure 1.6, illustrates a conventional cellular wireless system layout, where $A, B, C, D$ and $F$ are co-channel cells and $B S_{0}$ is the reference or desired cell.

Now these preceding technical challenges and associated bottlenecks, which extend across all aspects of wireless communication system design, including network de- 
sign with cross-layer protocol design, wireless terminal and circuit design with minimum power consumption, are clearly undesirable for the overall wireless system design.

\subsection{Motivation for the Research Work in the Thesis}

The demand for higher data rates in wireless networks is unrelenting, and hence has triggered the design and development of novel techniques by which system information capacity can be increased, at the same time maintaining high-quality of service. Cellular systems are partly used in wireless communication network to increase the information capacity of the network, by increasing the limited radio resource utilization. Other methods such as combination of bandwidth efficient coding/modulation techniques at the communication link level and the use of sophisticated channel allocation schemes are also being employed to increase the information capacity of wireless network.

The continuous growth in wireless capacity has led to shrinking of cell size radius, usage of higher carrier frequencies and employment of high frequency reuse in modern and emerging cellular systems [23, 37-39]. Modern and emerging cellular systems are therefore becoming increasingly interference limited, and in the center of Tokyo a reduction of cell size radius to $300 \mathrm{~m}$ or less has led to a problem of co-channel interference [1]. Smaller cell radius sizes increase the information capacity of the cellular wireless system, but an increase in co-channel interference may lower the information capacity increase [40]. Ranges of cell size for information capacity increase in cellular system have been reported in [4-6], but Zhou et al. in [7], has suggested that the may be a limit to cell size radius reduction for information capacity increase in cellular wireless communication systems. However, till now there is no quantify theoretical limit to cell size radius reduction. Also decreasing cells size means more cells are required for a given coverage area, which is always expensive, because of capital expenditure and operational expenses of the network [41, 42]. However there is no cost - information capacity performance criterion analysis for cell size reduction.

The number of co-channel interfering cells in the first and second tier has been given $[43,44]$, but there are no explanations or proof for how they were derived. Currently, conventional cellular system analysis is based on the assumption of first tier co-channel 
interference cells being active with other tier co-channel interfering cells being assumed to be negligible, because of the assumption of a large basic path loss exponent, lower microwave carrier frequency and bigger cell size radius [7].

Previous works leave unanswered the following fundamental questions:

- Why does co-channel interference become a problem when cell size radius gets smaller in cellular wireless networks?

- At higher microwave carrier frequencies, small basic path loss exponent and smaller cell size radius does other co-channel interference cell, other than the first tier cochannel interfering cells, become active?

- How can the number of co-channel interfering cells beyond the first tier be calculated?

- Is there a theoretical limit to cells reduction for information capacity increase in cellular wireless system in terms of network infrastructure cost?

Motivated by the preceding questions, this thesis seeks to address and find answers to them.

\subsubsection{Contributions of the Thesis}

The main contribution of this thesis is based on the key papers of the author [45-50] and other submitted work [51]. However, each chapter provides a detailed summary of contributions made within the chapter. Although the thesis is based on the key papers of the author, it also contains some previously published and unpublished results. The main contribution of this thesis is summarized as follows:

- It is shown geometrically, that the number of co-channel interfering cells in subsequent tiers, other than those in the first tier, is the product of the number of cochannel interfering cells in the first tier and the tier number, irrespective of the representation of the cellular pattern (equilateral triangles, squares or regular hexagons). 
- A two tier co-channel interference model is proposed for emerging and future cellular wireless communication systems. Mathematical analysis, supported by computer simulation, is used to show that at higher microwave carrier frequencies, second tier co-channel interference becomes active, as cell size radius and basic path loss exponent decrease.

- This is found by comparing the information capacity performance of a conventional cellular system (carrier frequency, $f_{c}<2 \mathrm{GHz}$ and cell size radii, $R$ $>1 \mathrm{~km}$ ), to that of an emerging cellular wireless system (frequencies $f_{c}>2$ $\mathrm{GHz}(3.35-15.75 \mathrm{GHz})$, cell size radii $R \leq 1 \mathrm{~km})$. The comparison was done using an existing one tier interference model and the proposed two tier interference model. The percentage decrease in information capacity $\left(\mathrm{b} / \mathrm{s} / \mathrm{Hz} / \mathrm{km}^{2}\right)$, between the two interference models at a carrier frequency, $f_{c}=900 \mathrm{MHz}$, cell size radius, $R=100 \mathrm{~m}$ and basic path exponent, $\alpha=2$, was $6 \%$. For a carrier frequency of $15.75 \mathrm{GHz}$, under the same cellular wireless system conditions the percentage decrease in information capacity was $15.32 \%$. Note that $\alpha$ is the basic path loss exponent for the propagation loss model.

- We argued that multiple tiers of co-channel interference may become active. Therefore, a multiple tier co-channel interference model was proposed. Mathematical analysis, supported by computer simulation, is used to show that, at higher microwave carrier frequencies as cell size radius and path loss exponent decreases, not only does the second tier co-channel interference become active, but multiple tiers of co-channel interference become active.

- This is established by comparing the information capacity performance of a cellular system, the existing one tier interference model and the proposed multiple tier interference model. At carrier frequency, $f_{c}=900 \mathrm{MHz}$, cell size radius $R=100$ and basic path loss exponent, $\alpha=2$, it was found that the percentage decrease in information capacity $\left(\mathrm{b} / \mathrm{s} / \mathrm{Hz} / \mathrm{km}^{2}\right)$, between the two interference model was $5.67 \%$. For a carrier frequency of $15.75 \mathrm{GHz}$, under the same cellular wireless system conditions the percentage decrease in information capacity was $36.41 \%$. 
- Analyses the impact of both:

1. system parameters (such as antenna height, operating frequency and reuse factor) and

2. propagation loss parameters (such as path loss exponent, breakpoint distance and effective road height),

on the information capacity performance of cellular wireless network operating at higher microwave carriers frequencies where, (i) two tier co-channel interference is active (ii) multiple tiers of co-channel interference are active.

- It is established that, for both the two tier and multiple tier interference model the information capacity performance of the cellular network is affected by the system and propagation loss parameters. For example, (i) in the case of the two tier interference model, at cell radius $R=100 \mathrm{~m}$, carrier frequency, $f_{c}=15.75 \mathrm{GHz}$, for extra path loss exponent, $\rho=2$. The percentage decrease in information capacity was $23.33 \%$, while for the same cellular system condition, when extra path loss, $\rho=8$, the percentage decrease in information capacity was $16.9 \%$ (ii) in the case of the multiple interference model, for the same cellular wireless system condition, the decrease in information capacity, when extra path loss, $\rho=2$, was $45.18 \%$, and for extra path loss, $\rho=8$, the percentage decrease in the information capacity was $24.34 \%$.

- It is shown, that because multiple tiers of co-channel interference are becoming active, there is a theoretical limit to cells radius size reduction in cellular wireless systems, for information capacity increase, founded on economic reasons.

- This is established, because at a carrier frequency, $f_{c}=15.75 \mathrm{GHz}$. The information capacity per unit cost [Bits/sec/Hz/unit cost], at cell size radius $R=$ $180 \mathrm{~m}$, for the conventional single tier interference model, equals that of the proposed multiple tier interference model at cell size radius $R=530 \mathrm{~m}$. 


\subsection{Organization of the Thesis}

The thesis aims at finding a theoretical limit to cell size reduction in cellular wireless communication system. The analysis focuses on two wireless channel models: multipath and cellular. For them, area spectral efficiency and economic efficiency are presented. Chapter 2, gives a brief overview of cellular wireless communication concept, wireless channel models and introduces the mathematical background to follow our derivations. The research contributions are presented in Chapters $3-6$. Chapter 7, presents conclusions and outlines future work.

Figure 1.7, is a roadmap of some routes that one might decide to follow through the coming chapters, and the territory that one would cover.

\subsubsection{Outline of the Thesis}

The chapters of this thesis are outlined as follows:

- Chapter 2 gives a brief overview of cellular wireless communication system concepts and frequency planning in cellular wireless system. The chapter also introduces the necessary mathematical background to follow the derivations and system models for the wireless and cellular channels. It reviews the link between cellular wireless system, propagation model, co-channel interference, cell size reduction and information capacity performance analysis.

- Chapter 3 proposes a formula for calculating the number of co-channel interfering cells in a given tier of a cellular wireless site layout. The formula is used for the characterization of co-channel interference at higher microwave carrier frequencies and smaller cell size radius. A co-channel interference model which incorporates the first and second tier co-channel interfering cells is also proposed.

- Chapter 4 analyzes the information capacity performance of a cellular wireless communication system. It compares the conventional interference model information capacity performance to that of our proposed two tier co-channel interference model information capacity performance. Mathematical analysis supported by computer simulation is used for the comparison. The impact of system and propagation loss 
parameters on the information capacity performance for the two interference models is studied.

- Chapter 5 proposes a multiple tier co-channel interference model. Mathematical analysis and computer simulation are then used to study the information capacity performance. The information capacity performance is studied by comparing that of the conventional interference model to the proposed multiple tier interference model. Next, the impact of system and propagation parameters on the information capacity performance for the two interference models is studied.

- Chapter 6 uses the information capacity - cost analysis to find a theoretical limit to cell size reduction in cellular wireless systems operating at higher microwave carrier frequencies. We also elaborate on the current development in cellular mobile communication technologies, which are relevant this research work.

- Chapter 7 summarizes conclusions drawn from the preceding chapters and points to future directions of this thesis. 


\section{Chapter 2}

\section{Overview of Cellular Wireless System}


The cellular wireless network evolved as the basic structure for any wireless communication system. The cellular system allows the reuse of wireless channels on the same carrier frequency at spatial located distance. The reuse of the wireless channels is advantageous for frequency band utilization [38]. The cellular system is therefore used for this research work. In a cellular wireless network all the serviced area is divided into a large number of small areas called cells. Each cell is serviced by its own radio-communication unit called the Base Transceiver Station (BTS). The cell is said to be served by a base station, which is stationary. The wireless channel poses a major challenge as a medium for consistent high speed communication, because of interference from co-channel cells [52]. In the thesis, our focus will be to model co-channel interference accurately for emerging and future cellular wireless systems. The propagation characteristics of a cellular wireless system also changes with the cell size radius [2]. We will therefore study the impact of propagation loss parameters on the information capacity of cellular wireless systems as the cell size radius reduces. This chapter, discuss the cellular wireless concept, system models for wireless channels and conventional methods used for increasing the information capacity of cellular wireless system.

The chapter is organized as follows. An overview of the cellular wireless concept is presented in Chapter 2.1. Next, we look at the geometrical properties of cellular wireless systems in Chapter 2.2. The wireless channel and channel impediments are discussed in Chapter 2.3 briefly. Next, a survey of work on the modelling of propagation losses is presented in Chapter 2.4. Followed by a discussion on cellular wireless channel in Chapter 2.5. Followed by a description of conventional interference and information capacity model for cellular wireless system in Chapter 2.6 and 2.7. Finally, a summary of the chapter is presented in Chapter 2.8.

\subsection{The Cellular Wireless Communication Concept}

The Bell System planners conceived the cellular wireless communication concept under the AMPS standard in 1979. They were looking ahead to a more economical and widespread form of mobile-telephone service [4]. AMPS was to provide a large-scale mobile-telephone service. The AMPS was based on interests of the public, mobile-telephone 
customers, and mobile-telephone operating companies.

The basic objectives of AMPS is as follows [4]:

- Large subscriber capacity

- Efficient use of spectrum

- Nationwide compatibility

- Widespread availability

- Adaptability to traffic density

- Service to vehicles and portables

- Regular telephone service and special services, including "dispatch"

- "Telephone" quality of service

- Affordability.

In a view that the AMPS system must be able to grow and accommodate larger subscriber capacity within a local service area, such as the environments of a single cities. The provision of service must not depend on the continuous enlargement of the allocated radio spectrum. The need for wireless systems to operate and grow within given radio channels was the driving force behind the evolution of the cellular concept [4].

Basically, the mobile network model of the Bell Systems under the AMPS standard in 1979 [4], consists ideally of hexagonal cells, the cells representing geographic areas. Base stations in each cell communicate simultaneously with all mobile stations. Traffic is passed through a radio network interface to an infrastructure of switching equipment called Mobile Switching Centres (MSC). The mobile switching centre interconnects the different parts of the communication system. The MSC is in turn connected to the public switch telephone network. Figure 2.1 illustrates the basic concept of the cellular wireless communication system. The triangles represent base stations. The basic elements of the cellular wireless system are frequency reuse and cell splitting concept. This concepts are discussed briefly in the next section. 


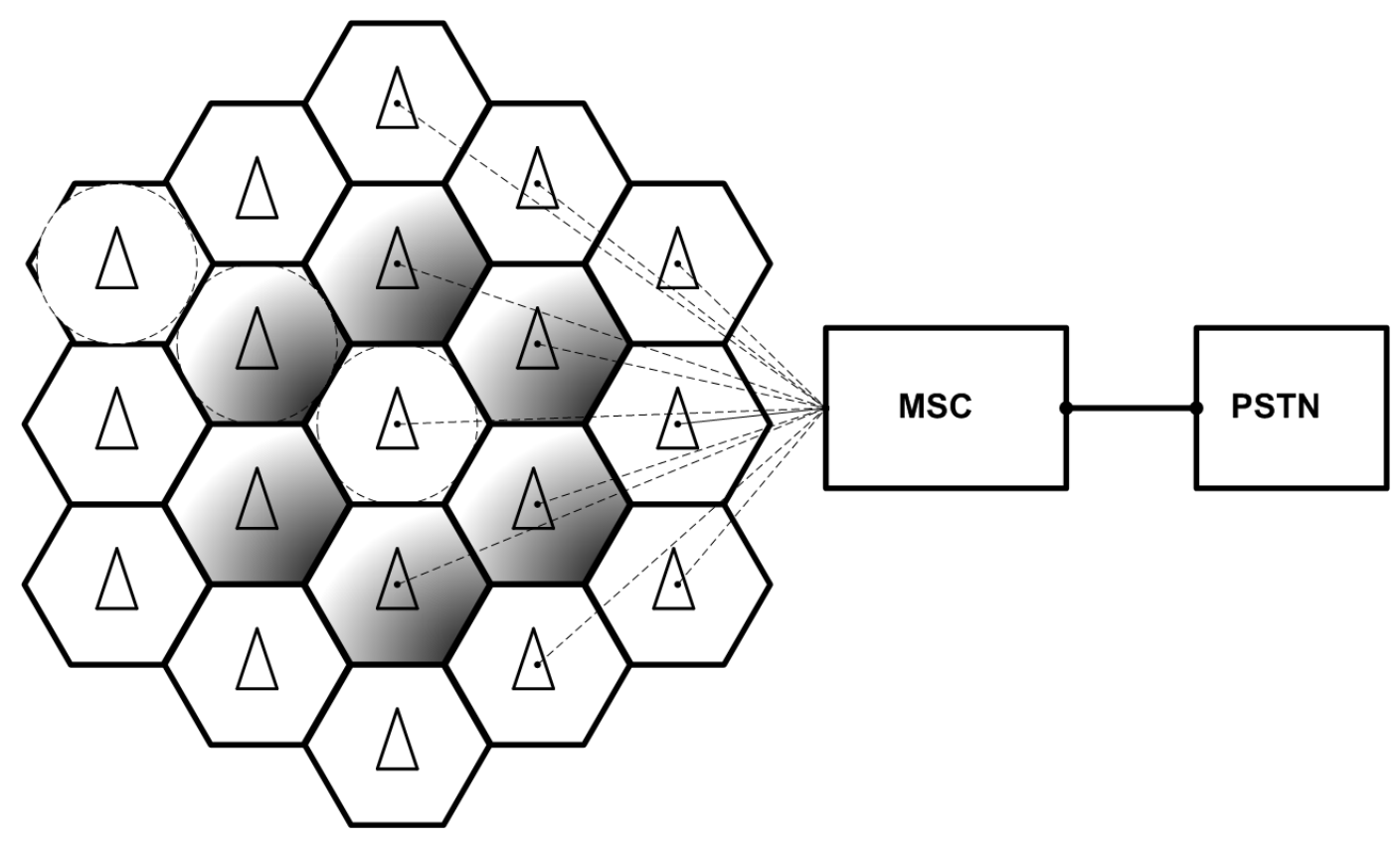

Figure 2.1: A cellular wireless network.

\section{Frequency Reuse in Cellular System}

Frequency reuse is the use of the same carrier frequency $f_{c}$ to cover different geographic areas. The different geographic areas are separated from each other by a sufficient distance so that co-channel interference does not become a problem. Frequency reuse is not only employed in cellular wireless communication system but also in broadcasting and most other radio services. Frequency reuse allows wireless communication providers to cover local areas with transmitters of moderate power as compared to a single land transmitter site with high power at a high elevation. Frequency reuse increases the information capacity for the cellular wireless network for the same number of allocated channel frequencies.

\section{Cell Splitting in Cellular System}

Cell splitting is one of the various methods used to increase the information capacity of a cellular wireless communication system. Cell splitting allows the revision of cell boundaries such that the areas regarded as large cell (single cell) can contain several smaller cells, and utilize all these cells channels. The revision of cell boundaries by cell splitting allows 
the cellular system to adjust to growth in information capacity demand in certain areas, or in the whole wireless network, without any increase in the spectrum allocation. The area of a cell for a cellular wireless communication system is proportional to the square of the cell size radius $\left(R^{2}\right)$. Therefore for example reducing the cell radius to one half of its original value causes the cell area to drop a one quarter of its original value. Theoretically, four of the smaller cells may fit into one of the large cells. However, for a hexagonal cell model layout it is impossible to fit four quarter - size hexagonal cells completely in a full - size hexagonal cell without some regions failing to be covered. The regions which are not covered by cell splitting are covered by adjacent cells.

\subsubsection{Frequency Reuse Concept in Cellular System}

Channel frequency distribution in a cellular wireless communication system depends on several parameters, such as signal propagation characteristics, signal interference and cellular site geometry. In cellular systems the total number of channels allocated to a network operator is divided into sets. Each set is then assigned to a cell inside a cluster of cells, which forms a pattern. This set of channel is then reused in another cell some distant apart to ensure acceptable signal interference.

The cells, using the same channels, are called co-channel cells. The distance between co-channel cells is called co-channel reuse distance, $D$. Note that the pattern formed is reused according to the co-channel reuse distance. The selection of the number of cells per cluster is based on the level of co-channel interference.

A better understanding of signal propagation and cellular site geometry is required to understand the performance and channel assignment of cellular wireless communication systems. In the following section we give a brief introduction to cellular site geometry and wireless channels, which we used for the derivation of the formula for finding the number of co-channel interfering cells in subsequent tiers. The formula is used for the information capacity performance analysis. 


\subsection{Properties of Cellular Wireless Geometry}

The reason for defining cells in a cellular wireless communication system is to demarcate areas in which either specific channels or a specific cell site will be used at least preferentially, if not exclusively [4]. In cell site geometry a realistic degree of geographical confinement of channel usage is necessary to prevent co-channel interference.

Cell-site selection and position tolerance is an important parameter in cellular wireless communication networks, likewise the maximum and minimum cell size radius as reported by [4]. The values for these parameters are founded on the tradeoffs between information capacity, cost effects and good transmission quality. A perfect cellular wireless communication network layout model is supposed to be regular. However the installation of a cell site in the ideal position is generally not possible. The AMPS systems allow the position of cell sites up to one-quarter of the nominal radius away from the ideal location.

The value for maximum cell size radius, is a decision taken during the start up phase of a cellular system. It is a compromise between cost, forecast of the ultimate information capacity and the transmission quality required. The maximum cell size radius, is defined by, (i) the cost of transmitter power and antenna height (ii) the expected number of cell sites in a mature cellular system (iii) signal propagation environment characteristics, and (iv) the maximum power level of mobile terminal [4]. The maximum cell size radius has only an indirect effect on the system objective of a large ultimate capacity [4].

The minimum cell size radius comes into play in a mature cellular wireless communication system. In terms of cost, it may have little effect on the customer, but will have a significant effect on the information capacity of the cellular wireless system. The practical obstacles which affect smaller cell size are the cell site position tolerance, and co-channel interference. In this thesis we will focus on the co-channel interference.

\subsection{Wireless Channels}

From a technical point of view, the distinction between wireline, and wireless communication is the physical properties of the wireless channel. These physical properties pose a major challenge to the use of wireless channel as a reliable medium for high-speed 


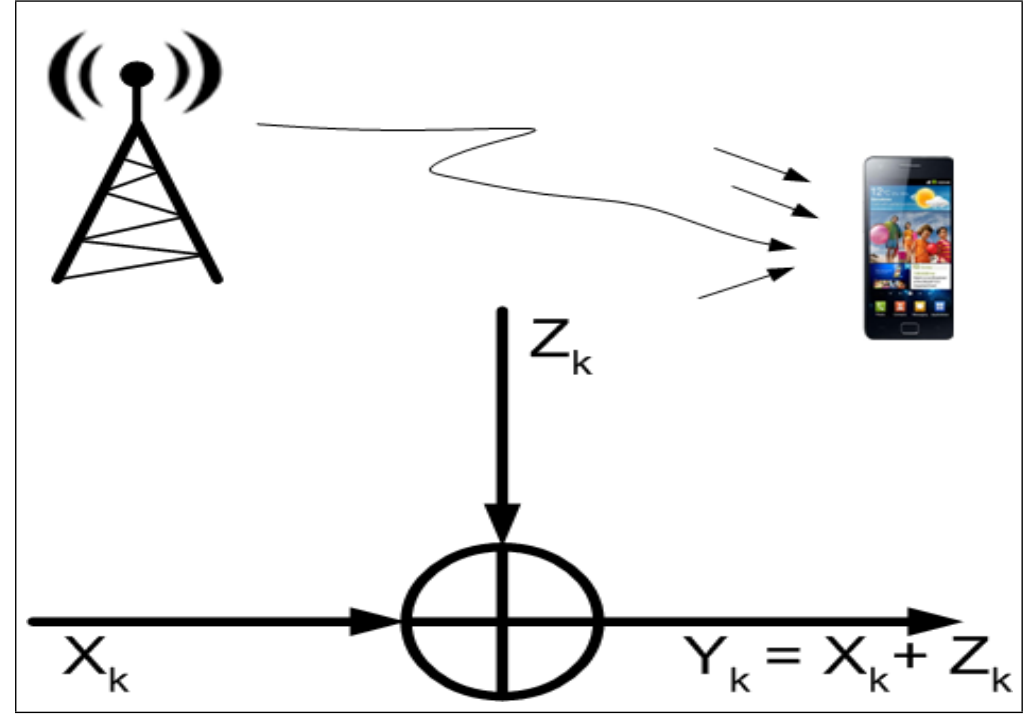

Figure 2.2: Basic additive Gaussian noise channel.

communication, which is one of the requirements needed to achieve the wireless future development direction. The physical properties to which wireless channel are vulnerable to, are: ambient noise, propagation losses, interference and multipath signal propagation. Wireless channel are also vulnerable to properties arising from the use of multiple antennas. The following subsections will review these physical properties briefly, however further discussion and details can be found in $[53,54]$.

\subsubsection{Additive Noise Channels}

Wireless channels are corrupted by ambient noise, which is normally introduced by the hardware components at the receiver front end of the wireless communications system. The noise is from thermal motion of electrons on the antenna, receiver electronics and background radiation sources. This noise is modelled as having a very wide bandwidth. A common model for such a noise is a zero-mean Gaussian process. Figure 2.2, illustrates the additive noise channel, where $\mathrm{Z}_{k}$ is the additive noise, $\mathrm{X}_{k}$ is the transmitted signal and $\mathrm{Y}_{k}$ is the received signal. The channel is referred to as additive white Gaussian noise (AWGN)) when the noise is white. AWGN model is referred to as an idealistic channel condition (it is not a real physical process) where no signal fading occurs, because it is assumed that its spectrum density is constant over all frequencies. Now since receiver hardware always 
introduces some noise, models for any time-varying channel must include an additive noise term, unless the noise is negligible relative to other channel impediments. In this thesis we assumed noise to be negligible relative to the other channel impediments, which will be discussed in the subsequent chapters of the thesis. Transmission over additive Gaussian noise channels has been studied over the past decades in [25, 55-57]. In the next section, we consider multipath effects, which can cause two types of signal degradation in cellular wireless communication systems.

\subsubsection{Multipath Signal Propagation Channels}

Multipath signal propagation, in wireless communications refers to the phenomenon in which multiple copies of a transmitted signal are received at the receiver. Unlike the multiple-input multiple-output (MIMO) system which uses multipath propagation to provide higher capacity $[58,59]$, for classical systems multipath signal propagation is considered to be harmful. Therefore research work that followed the viability of wireless communication system had at least two phases: understanding the nature of multipath channels and searching for techniques to reduce signal fading. In this section a detailed overview of multipath channels and its modelling is given. In wireless radio environment, particulary urban environment, because of the surrounding structures a radio signal transmitted from a fixed source to a mobile receiver experiences an excessive variation in both amplitude and phase. This variation is due to multiple paths which are created after the transmit wave reflects, diffracts or scatters by human-made structures along the path of propagation [60], as illustrated in Figure 2.3. The transmitted waves travel in different directions; thus forming additional copies of the transmitted signal which can be attenuated in power, delayed in time, shifted in phase and/or frequency from the LOS signal path. The received signals can sum-up constructively or destructively (constructive and destructive interference). Thus, the summed-up signal received can be strong in one area but weak in another. The mobile receiver can also be situated in a signal null spot. The raising or lowering of the received signal at the mobile unit depends on the location of the standstill mobile [61]. When the transmitter, receiver, and reflectors are all static, then the constructive and destructive interference of the multiple paths and their delays relative to 


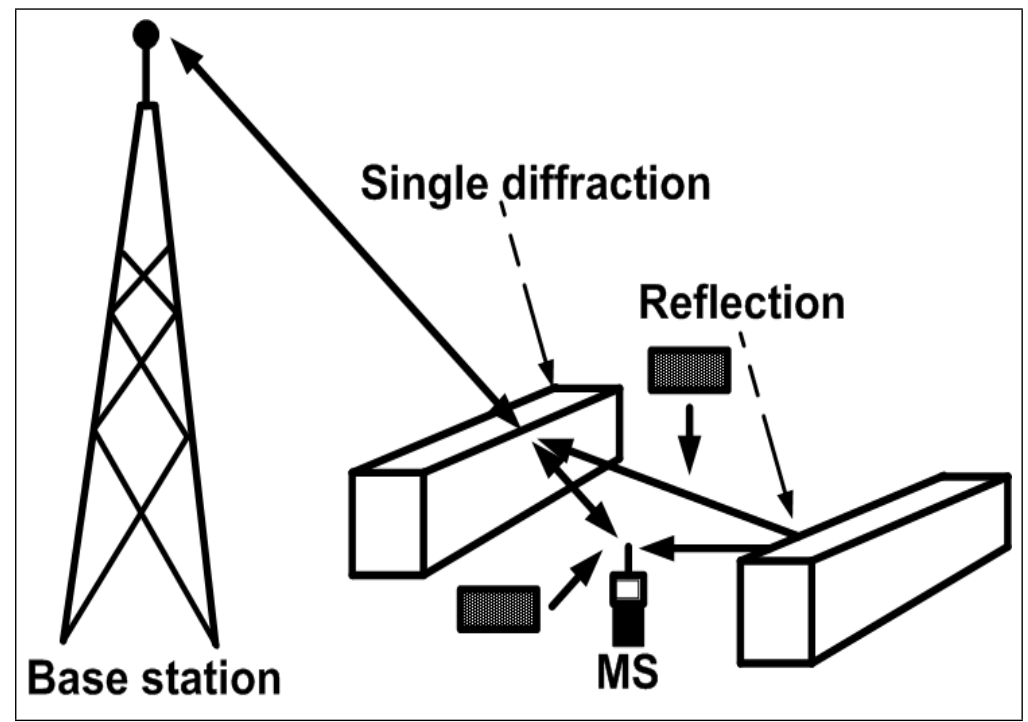

Figure 2.3: Distribution of single diffraction, reflection and scatters in a wireless communication environment.

the LOS path, are fixed. However, when the source or receiver is not static, then the characteristics of the multiple paths vary with time. These time variations are deterministic when the number, location and characteristics of the reflectors are known, otherwise, statistical models must be used. In the following section propagation loss and site-specific models for path loss will be discussed. In discussing the models, distances are assume to be small enough not to be affected by the Earth curvature [60].

\subsection{Propagation Losses}

Before implementing designs and confirming the planning of wireless communication systems, it is necessary that accurate propagation characteristics of the environment are known [62]. Propagation prediction usually provides two types of parameters corresponding to large-scale path loss (diffusive losses) and small-scale (shadowing) fading statistics. The path-loss information is important for the determination of coverage of a BS placement and its optimisation. The small-scale parameters provide statistical information on local field variations and this, in turn, leads to the calculation of important parameters that help improve receiver $(\mathrm{Rx})$ designs and combat the multipath fading. Without propagation predictions, these parameter estimations can only be obtained by field measurements 
which are time consuming and expensive.

Losses due to propagation are also an issue in wireless channels. Diffusive losses occur because of the nature of the wireless channels. The energy radiated by a simple point source in free space spreads over an ever-expanding spherical surface as the energy propagates away from the source. That is, an antenna with a given aperture size will gather an amount of energy which decreases with the square of distance between the antenna and the source. In land wireless channels, the diffusion losses are normally greater than this, because of the effects of ground-wave propagation, foliage, etc. In land cellular mobile networks the diffusion loss is inverse-square with distance within LOS of the cell tower, and it falls off with higher power (3 or 4 ) at greater distances. Shadow fading is due to the presence of objects such as buildings, walls and trees between transmitter and receiver. It is modelled by an attenuation (that is a multiplicative factor) in signal amplitude which follows a lognormal distribution. Variation in fading is represented by the standard deviation of the logarithm of the attenuation. The propagation path loss of a signal is a function of factors such as the environment, antenna height and antenna type.

The path-loss prediction models can be roughly divided into three types, that is, empirical, theoretical, and site-specific models [62]. The empirical models are a set of equations derived from extensive field measurements [63]. Empirical models are simple and efficient to use. They are accurate for environments with the same characteristics as those where the measurements were made. The input parameters for the empirical models are usually qualitative and not very specific. A disadvantage of the empirical models is that they cannot be used for different environments without modification.

\subsubsection{Ray-Tracing Technique}

Ray tracing is a technique, which is based on Geometrical Optics (GO) and it can be applied as an approximation method for estimating the levels of high frequency electromagnetic fields. Ray theory is a procedure for providing an accurate site-specific means to obtain useful simulation results $[64,65]$. This technique also serves as a starting point for statistical modelling $[66,67]$. Ray-tracing method unlike other computational complex modelling tools, is simple. However, it must be stated that it can be computationally 


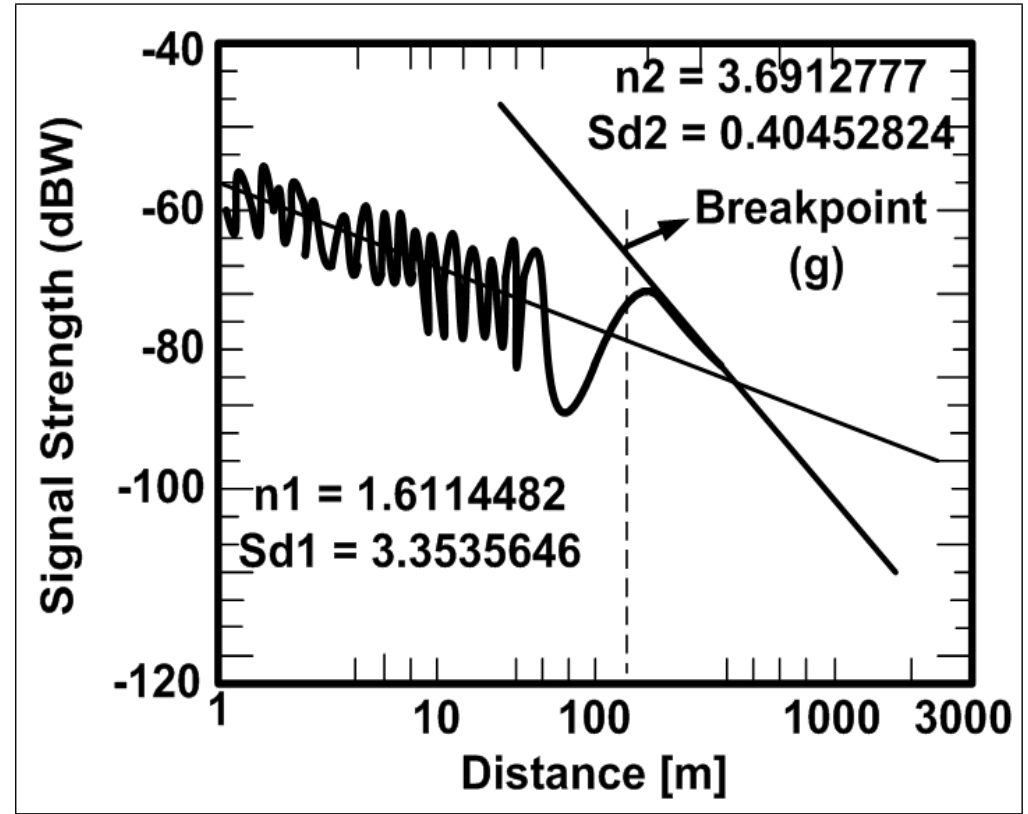

Figure 2.4: Illustration of multiple slope regression fit to the two-ray model [68].

inefficient.

\subsubsection{Two-Path Model}

The two-path model is a simple and measurement-based model and is used for line-ofsight (LOS) propagation in an urban area. The model is based on a two-ray propagation mechanism, which predicts the signal variation when a single ground reflection dominates the multipath signal propagation effects [63]. This model is characterized by the fact that a breakpoint exists that clearly separates the different properties of propagation in near and far regions relative to the BS, as shown in Figure 2.4. When the regression analysis was used for the measured data in the San Francisco Bay area, it was shown that the slope before the break point is less than two, while that after the breakpoint is greater than two [68]. The two-ray model for LOS propagation was extended in [69] to include the effects of traffic and high obstacles such as electric poles and vehicular traffic. It is shown that when the heights of car traffic and some obstacles are included in the model, better model accuracy can be obtained compared with the experimental results. Figure 2.5 shows the geometry and notation for the two-path model. The figure shows that the received signal at the mobile station consists of two components: the direct or LOS components, 


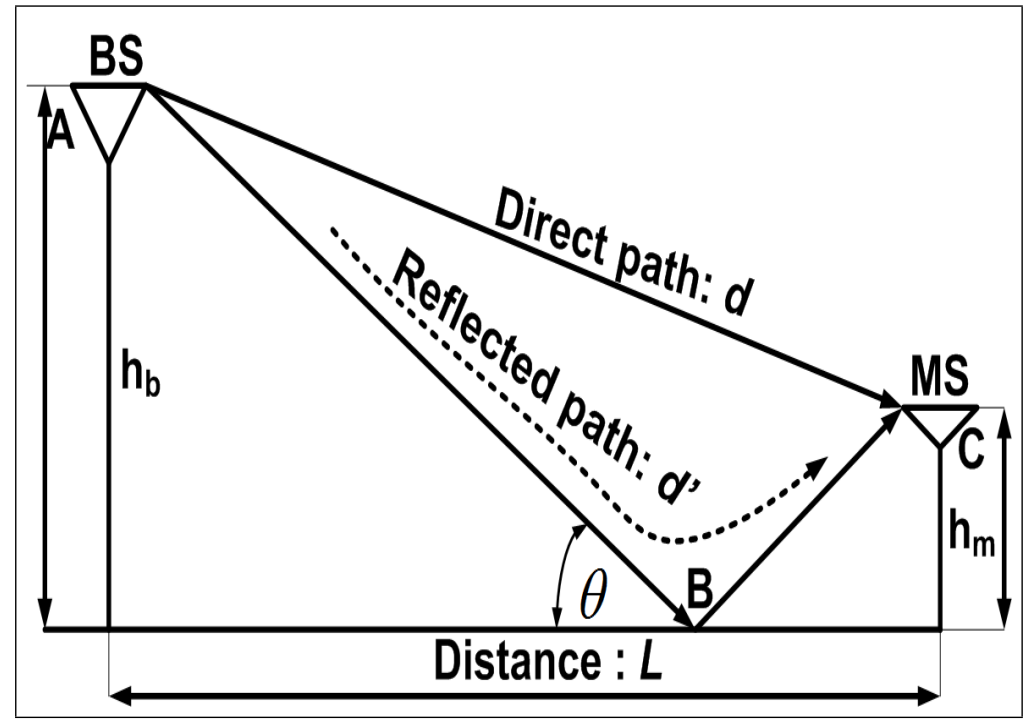

Figure 2.5: Two-path model showing direct and reflected path for propagation.

which represent the transmitted signal propagating through free space, and a reflected component, which is the transmitted signal reflected off the ground. Other two-path model parameters are defined as follows (see also Figure 2.5):

- L : distance of separation between transmitter and receiver

- $d$ : length of the LOS path

- $\mathrm{d}^{\mathrm{s}}$ : length of reflected path

- $\Gamma$ : ground reflection coefficient

The received LOS component at the MS is found from the free-space path loss formula:

$$
r_{L O S}(t)=u(t) \frac{\lambda G_{d} e^{j(2 \pi d / \lambda)}}{4 \pi d},
$$

where $u(t)$ is a complex baseband signal, $\lambda$ is the carrier wavelength, $d$ is the horizontal separation distance between transmitter and receiver antennas. Gain $G_{d}$ is the product of the transmitter and receiver antenna field radiation patterns in the direct path. If the effect of surface wave attenuation is neglected, because of the assumption that the antennas are located more than a few wavelengths from the ground then by superposition, the received 
signal for the two-path model can be written as:

$$
r_{\text {twopath }}(t)=\frac{\lambda}{4 \pi}\left[\frac{G_{d} u(t) e^{j(2 \pi d / \lambda)}}{d}+\frac{\Gamma G_{d^{\prime}} u(t+\tau) e^{j\left(2 \pi d^{\prime} / \lambda\right)}}{d^{\prime}}\right],
$$

where $\tau=\left(d^{\prime}-d\right) / \lambda_{c}$ is the time delay of the ground reflection, $\Gamma$ is the reflection coefficient [70]. Gain $G_{d^{\prime}}$ is the product of the transmit and receive antenna field radiation patterns of the reflected paths. For a transmitted signal which is narrowband relative to the time delay $\left(\tau<<B_{u}^{-1}\right)$, where $B_{u}$ is the complex baseband signal bandwidth, then $u(\mathrm{t})$ $\approx u(\mathrm{t}+\tau)$. Therefore the received signal power for the two-path model for a narrowband transmission is given as:

$$
S_{r}=S_{u}\left[\frac{\lambda}{4 \pi}\right]^{2}\left|\frac{G_{d}}{d}+\frac{\Gamma G_{d^{\prime}} e^{j \phi}}{d^{\prime}}\right|^{2}
$$

where $S_{u}$ is the complex baseband signal power, $\phi$ is the phase difference between the two received components (that is the direct and reflected paths). If $L$ denotes the horizontal distance between the transmitter antenna of height $h_{b}$ and the receiver antenna of height $h_{m}$, then the phase difference $\phi$ is given by:

$$
\phi=\frac{2 \pi\left(d^{\prime}-L\right)}{\lambda}=\frac{2 \pi}{\lambda}\left[\left[\left(\frac{h_{b}+h_{m}}{L}\right)^{2}+1\right]^{1 / 2}-\left[\left(\frac{h_{b}-h_{m}}{L}\right)^{2}+1\right]^{1 / 2}\right] .
$$

The equation (2.3) has been shown to agree closely with empirical data from the work in [71]. Basically the delay spread of the two-path model is the excess delay of the ground reflection: $\left(d^{\prime}-d\right) / f_{c}$. For $L>5 h_{b} h_{m}, d^{\prime}-d \approx 2 h_{b} h_{m} / L$, therefore $\phi \approx 4 \pi h_{b} h_{m} / \lambda L$.

The ground reflection coefficient is given by $[60,70]$

$$
\Gamma=\frac{\sin \theta-Z}{\sin \theta+Z}
$$

where $\mathrm{Z}$, the characteristic impedance of the media, is given by

$$
Z= \begin{cases}\sqrt{\epsilon_{r}-\cos ^{2} \theta} / \epsilon_{r} & \text { for vertical polarization } \\ \sqrt{\epsilon_{r}-\cos ^{2} \theta} & \text { for horizontal polarization }\end{cases}
$$

$\epsilon_{r}$ is the dielectric constant of the ground, which for earth or road surfaces is approximately that of a pure dielectric $\left(\epsilon_{r}=15\right)$. From equation (2.4), if $L>5 h_{b} h_{m}, d^{\prime}-d \approx 2 h_{b} h_{m} / L$, and hence

$$
\phi \approx \frac{4 \pi h_{b} h_{m}}{\lambda L}
$$


for asymptoically large $L, d^{\prime} \approx d \approx L, \theta \approx 0, \mathrm{G}_{d} \approx \mathrm{G}_{d^{\prime}}$, and $\Gamma \approx-1$. Now substituting these approximations into equation (2.3), it is seen that in this asymptotic limit, the received signal power is approximately

$$
S_{r} \approx\left[\frac{\lambda G_{d}}{4 \pi L}\right]^{2}\left[\frac{4 \pi h_{b} h_{m}}{\lambda L}\right]^{2}
$$

Therefore, in the asymototic limit of large $L$, the recived signal power falls off inversely with the fourth power of $L$. In [72], plots of received signal power (equation (2.3)) as a function of distance show this asymptotic limit. Up to a certain critical distance $d_{c}$, the wave experiences both constructive and destructive interference from the two rays, which results in a wave pattern with a sequence of maxima and minima.

\section{Critical Distance}

The critical distance $d_{c}$, is the final maximum reach by the two rays, after which the signal power falls off inversely proportionally to $r^{4}$. An approximation for $d_{c}$ can be obtained by setting $\phi$ to $\pi$ in equation (2.6), obtaining $d_{c}=4 \mathrm{~h}_{b} \mathrm{~h}_{m} / \lambda$. Note that the critical distance is used in the design of cellular wireless communication systems to determine optimal cell size [22], hence, we used it in the analysis for finding the minimum cell size for information capacity increase in cellular wireless networks.

If the local maxima and minima are averaged out from the equation (2.3), the resulting average power loss can be approximated by dividing the power loss curve into two regions (region I and II). For $L<d_{c}$, the average power falloff with distance corresponds to free space loss. For $L>d_{c}$, the falloff with distance is approximated by the fourthpower law as in equation (2.7). These approximations, thus, capture the simplified model for the average received signal power presented in [71], which we used to calculate our received signal power in this thesis.

\subsubsection{Statistical Fading Models}

A brief description of the statistical model for the received signals is given in this section. The statistical model will enable future work on this thesis to incorporate signal fluctuations. There are generally two phenomena that cause fluctuations of the received signal as 
the receiver or transmitter moves. As discussed in the previous section, multiple reflected signals arrive at the receiver shifted in phase. The received signals cause constructive or destructive interference. The resulting variations in the signal amplitude, called signal fading, vary over distances proportional to a signal wavelength. This type of fading is referred to as fast fading. When the number of multipath signal components is large, the law of large numbers can be used to approximate the fast fading effects with Gaussian statistics, which can be described by the Rayleigh statistics of the short-term signal envelope variation [22].

In addition to interference effects, as stated in previous sections the LOS and reflected paths may also be attenuated by buildings or other objects. This type of fading, or shadowing, varies over distances which are proportional to the size of the buildings, and is thus referred to as slow fading. When the number of signal attenuators is large, a Gaussian approximation for the attenuation distance can be used for the slow fading statistics; this results in a log-normal distribution for the signal variation over large distances.

The statistical model for short-term multipath fluctuations of the received signal amplitude is normal founded on a physical propagation environment consisting of a large number of isolated reflectors with unknown locations and reflection properties. Likewise for the long-term multipath fluctuations, since the phases rotate $\pi$ degrees every $1 / 2 \lambda$, the signal amplitude changes rapidly over short distances. If these local variations are averaged out, the local mean will also vary with distance due to two effects: the propagation loss with distance described above for the ray tracing models, and the changing configuration of surrounding buildings and obstacles which attenuate both the LOS and the multipath components. Based on the two- and ten-ray models, it is generally assumed that the propagation loss with distance is proportional to $r^{-2}$ in a rural environment, and $r^{-4}$ in an urban environment.

\subsubsection{Site-Specific Models for Path Loss}

Site-specific propagation models, also referred to as deterministic models, are based on the theory of electromagnetic wave propagation. While statistical models depend on extensive measurements, site-specific propagation models do not rely on extensive measurements, 
but on information of the environments, and they provide an accurate predictions of the signal propagation [73]. In theory, propagation characteristics of electromagnetic waves can be calculated exactly by solving Maxwell's equations. However, this approach requires very complex mathematical operations and requires considerable computing power.

\subsection{Cellular Wireless Channels}

The demand for high speed data service wireless communication systems, means the limited available frequency spectrum needs to be used efficently. Cellular systems exploit the power falloff with distance of a transmitted signal to reuse the same frequency channel or time slot at another spatially separated location [63]. The coverage area is divided into cells where, in each cell, only one user is assigned to a particular channel or time slot. For frequency division, the total system bandwidth is divided into orthogonal channels centered around a frequency, and each frequency channel is reused at a spatially separated cell. For time division, the signal occupies the entire frequency band, and is divided into time slots which are reused in distant cells. In this thesis we considered a time division cellular wireless communication system, because it is the most representative of the cellular wireless communication system.

Normally, for a cellular wireless system, the shape of the cell is determined by the power footprint of the transmitting BS, which is circular if the transmit and receive antennas are isotropic and propagation follows a free-space loss model. However, urban propagation does not follow the free-space model, as blockage and multipath fading cause distortion of the circular shape. To achieve spectral efficiency it is required that the spatial separation of cells that share the same frequency channel or time slot must be as small as possible to cover the largest possible area with a single channel. However, as the spatial reuse distance shrinks, the interference from cells using the same frequency or time slot increases. To complicate matters further, both the transmitted and interfering signals experience the long- and short-term multipath fuctuations described in the previous section. To help determine the spatial reuse, data rates, and system layout, accurate models for cellular transmission and co-channel interference are required. That is the reason why in this thesis we propose a more accurate co-channel interference model for cellular wireless 
communication systems operating at carrier frequencies greater than $2 \mathrm{GHz}$. It should be noted that coverage areas can also be divided using code division and spread spectrum code division techniques [38]. However, it is beyond the scope of this thesis.

We now consider models for the two types of urban cellular wireless communication systems, which are founded on the cell size radius. Since propagation conditions in suburban and rural areas are more favorable than in cities, these urban models generally show worst-case propagation conditions. The first model is the urban macrocells. General macrocells are related to cells in city centers, where the base stations are placed on the tops of tall buildings, and transmit enough power to cover about $500 \mathrm{~m}-2 \mathrm{~km}$ [74].

Now, if all parameters scale with distance then by shrinking the size of a cell by a factor of $N$ we can accommodate $N$ times more users in a given area, since each cell accommodates the same number of users in a smaller area. However, in order to shrink the cell size radius to smaller size, the base stations must transmit at a much lower power than in macrocells, and therefore must be placed closer to the ground. From the previous section, we know that lower antenna placement fundamentally changes the mechanism of signal propagation. We therefore use a smaller cell size radius (microcells) model for the case when the transmitters antennas are less than $20 \mathrm{~m}$ high. Transmit power in microcells is generally sufficient to cover about a $300 \mathrm{~m}$; this cell diameter is chosen since it corresponds to the point at which the power falloff of a transmitted signal versus distance increases from $r^{2}$ to $r^{4}$, thereby significantly reducing the power from distant interferers.

In cellular wireless communication systems, there are two transmission links: MS to the BS, which we will refer to as the uplink and BS to MS which we will refer to as the downlink. The uplinks are separated in frequency from the downlink, so the BS's interfere with each other, but not with the MS's, and vice versa. From the previous sections, based on existing models [61] both empirical and analytical the interference is generally much greater than the receiver noise, therefore as stated, early receiver noise will be neglected in our system models and analysis. 


\subsubsection{Macrocells Wireless Channel}

The conventional macrocell model requires propagation characteristics for both the transmitted signal within the cell and the interference from other cells. Now building concentration in urban environment is dense, thus both transmitted and interfering signals are blocked or reflected from lots of objects. Therefore, the statistical propagation model describe earlier applies to macrocells. When the radiated power tends to form approximately circular contours (isotropic antenna), the long-term received signal variation in both direction of the transmission link is closely approximated by a free-space propagation model with additional log-normal shadowing. Thus, the received signal power, averaged over multipath fading is modelled by [63]

$$
S_{r}=S_{u} \frac{A V}{r^{\alpha}}
$$

where $S_{u}$ is the transmitted signal power, $A$ is median path loss at $r=1 \mathrm{~km}, r$ is the BS - MS separation (in $\mathrm{km}$ ), $\alpha$ is the propagation exponent and $V$ is a lognormal shadow fading variation, with typical variance for urban environment ranging between $3-8 \mathrm{~dB}$. For a macrocell a given area is covered with nonoverlapping cells, and hexagonal cell shape is used as the closest tessellating shape to a circle as shown early in Figure 1.6.

For the case of narrowband transmissions in a macrocell network, the short-term fluctuation of both the desired and interfering signal envelopes generally follows a Rayleigh distribution [22]. However, if the transmitted signal has a LOS path to the receiver then the fluctuation of the desired signal is Rician [75]. For the conventional macrocell the number of interfering signals is random, but normally only the interferences in the first closest ring (first tier) of cells are taken into account and all others are considered to be negligible as shown in Figure 1.6, for a macrocell site layout with a cluster size of seven. For the uplink, the distance between an interfering and a transmitting BS is known, whilst in the case of the downlink because MS may be anywhere within the cell the interfering power is a random variable. However, in this thesis our work is related to smaller cell size (microcell), cellular wireless networks, which we discuss in the following section. 


\subsubsection{Microcells Wireless Channel}

Generally smaller cell size radius cellular wireless communication systems are to provide a high spectral efficiency to counterbalance their large cost due to the number of BSs' required. For a microcellular network, there are two types of propagation: LOS propagation, which is propagation between BSs and MSs with direct path between them, and non-LOS propagation, which is the case where there is no LOS path. In the non-LOS propagation the signal must "bend" around an obstacle such as a corner or corners via diffraction, scattering, or reflection to reach the intended receiver. As discussed in earlier sections LOS propagation in microcells can be accurately modelled with the two-ray model. However, it is difficult to use ray tracing to model non-LOS propagation, because it requires detailed information about the building and street layout, geometry, and dielectric properties. This information requires field measurements for the particular cell of interest, and the resulting model will only apply to that particular cell site.

However, a more general model for non-LOS for cities with rectilinear street layouts was developed in [22]. This measurement-based model was obtained from data collected in Manhattan at $900 \mathrm{MHz}$. The model includes a prediction method for the mean average power, and a statistical model for both short-term and long-term variations about this mean. In this thesis we neglect non-LOS in our model, because users in the non-LOS condition represent less than $1 \%$ of the LOS interference [76].

\subsection{Interference in Cellular Wireless Systems}

In the previous sections we discussed that the spectral efficiency over a geographical area for partition techniques, such as FDMA/TDMA can be increased by reusing the same frequency or time slot at spatially located cells where the power falloff with distance minimizes the effect of intercell interference. The extent of the intercell interference depends on both the distance between the interfering transmitters and the intended receiver as well as the propagation laws governing the interferers' transmissions.

In a cellular wireless communication system model, interference distribution is normally assumed to be Gaussian. This assumption is reasonable for CDMA systems, as 
there are many intracell and intercell interferers, hence the Gaussian distribution follows from the law of large numbers. However, with FDMA or TDMA, there are only a few active interferers, therefore the white noise assumption is generally not valid. In information capacity calculations, Gaussian interference is a worst-case noise assumption. However, most cellular system are interference limited, which means that the receiver noise power is much less than the interference power, hence can be neglected [26]. If the cellular system is considered to be interference limited, which is the case in this thesis, then for multiple users, using the same carrier frequency the carrier-to-interference ratio $(C I R)$ is used to measure the amount of interference over a specified carrier. For conventional cellular wireless communication system the $(C I R)$ is given as

$$
C I R=\frac{C}{I}=\frac{C}{\sum_{n=1}^{N_{I}} I_{n}}
$$

where $C$ is the carrier power and $I$ the interfering power. $N_{I}$ is the number of co-channel interfering cells in the first tier. For the conventional cellular wireless system other tier co-channel interferences are neglected, which holds because carrier frequencies are less than $2 \mathrm{GHz}$, and cell size radius are $1.6 \mathrm{~km},(1 \mathrm{mile})$ and above [4]. However, for emerging cellular wireless communication systems carrier frequencies are greater than $2 \mathrm{GHz}$, and cell size radii are less than $1 \mathrm{~km}[2,28,45]$. Therefore, there is a need to propose a new co-channel interference model for emerging cellular system, which is one of the aims of this thesis.

For the conventional cellular system where noise is neglected, the $C I R$ is given by [41]

$$
C I R=\frac{C}{I}=\frac{R^{-\alpha}}{\sum_{n=1}^{N_{I}} D^{-\alpha}}
$$

where $\alpha$ is the path loss exponent, $D$ is the frequency reuse distance, and $R$ is the radius of the cells, which is defined as the distance from the centre of the cell to any of its vertices. In the next section, we will define the area spectral efficiency, which quantifies the effect of co-channel interference on cellular wireless communication system information capacity. Area spectral efficiency is used in this thesis for our information capacity analysis. 


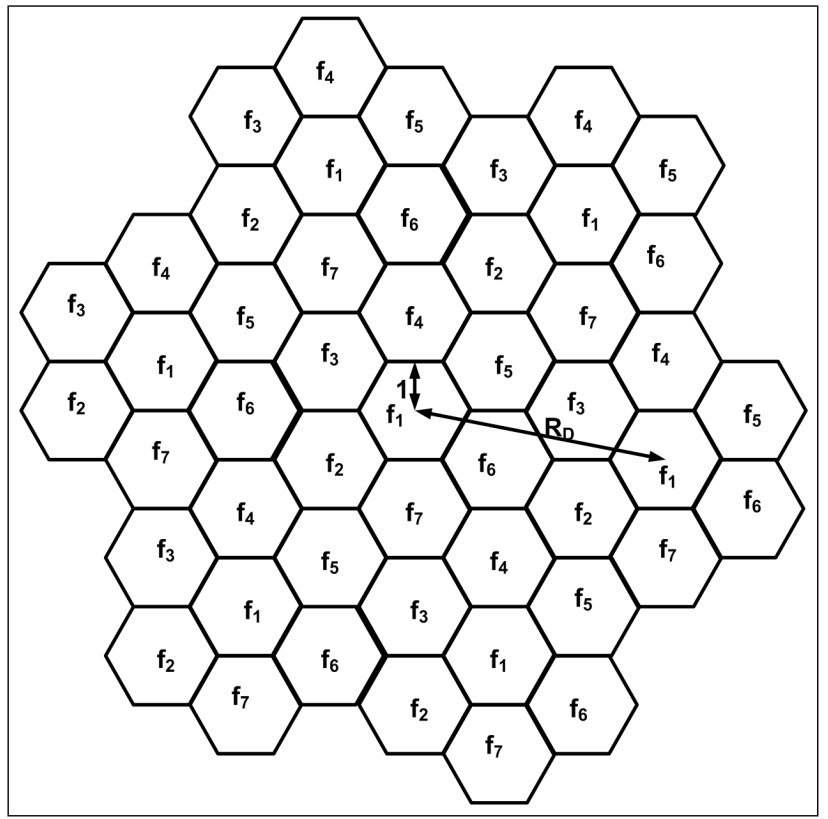

Figure 2.6: Reuse distance of a frequency division cellular wireless system showing frequency channels, $f_{1}, f_{2}, f_{3}, f_{4}, f_{5}, f_{6}$ and $f_{7}$.

\subsection{Cellular Wireless System Information Capacity}

In this section, we give a brief review of spectral efficiency and define area spectral efficiency, which we used for our information capacity performance analysis. The information capacity of a cellular wireless system is an important measure in designing and comparing the performance of wireless communication systems. The ultimate information capacity of a cellular wireless communication system is directly related to its spectral efficiency for a given quality of service [29]. The spectral efficiency of a cellular wireless system depends on a number of parameters, such as the number of radio channels required per cell and the interfering environment.

The spectral efficiency of a cellular wireless communication system can be expressed in a number of ways, such as Erlangs per square kilometer, number of users per square kilometer and the maximum average data rates per unit bandwidth per unit area. In this thesis, for the information capacity performance analysis, we have adopted the area spectral efficiency given by [77]. The area spectral efficiency of a cell is defined as the total bit rate/Hz/unit area that is supported by a cell's BS. This definition gives a more complete picture of the spectral efficiency by expressing it in terms of bit rates, bandwidth 
and area.

If a cell size radius is normalized to one, and the reuse distance $R_{D}$ is defined as the minimum distance between any two BSs that use the same code, frequency or time slot, since a code, time slot, or frequency is reused at a distance $R_{D}$ as illustrated in Figure 2.6, the area covered by one of these partitions is about $\pi\left(1 / 2 R_{D}\right)^{2}$. The area spectral efficiency is approximated by [77] as

$$
A_{e}=\frac{\sum_{k=1}^{N} R_{k} / B}{\pi\left(R_{D} / 2\right)^{2}}
$$

where $N$ is the total number of users per cell, $R_{k}$ is the data rate of the kth user, and $B$ is the bandwidth occupied by each user. The equation (2.11), shows that reducing the reuse distance, (that is reducing cell size radius) without altering $R_{k} \mathrm{~s}$, leads to an increase in information capacity. However, reducing the reuse distance increases intercell interference, because interference travels a shorter distance.

\subsection{Summary}

In this chapter, we have given a brief review of the cellular wireless concept, a description of frequency reuse, and cell splitting for information capacity increase in cellular wireless communication systems. We have also stated some of the impediments to reliable communication over wireless channels, which include multipath, shadowing, and interference. The two-ray tracing techniques, which specifically calculate the attenuation and phase of each received signal power path for urban areas founded on the geometrical configuration of the transmitter, receiver, and surrounding buildings was also described.

The chapter was concluded by defining the area spectral efficiency, which is used for the information capacity performance analysis as the data rate/Hz/unit area. The formula for the area spectral efficiency introduced includes the effect of interference in the data rate calculation. In the following chapter an inductive approach is used to generate a formula for calculating the number of co-channel interfering cells in a given tier of a cellular wireless communication system site layout. We also proposed a co-channel interference model for emerging and future cellular wireless communications system, which will be operating at carrier frequencies greater than $2 \mathrm{GHz}$ and smaller cell size radius. 


\section{Chapter 3}

Proposed Formula to Calculate Number of Co-channel Interfering Cells in Cellular System Site Layout, and Co-channel Interference Model for Two Tier Co-channel Cells 


\subsection{Introduction}

In this chapter, an inductive approach is used to generate a formula for calculating the number of co-channel interfering cells in a given tier of a cellular wireless communication system site layout. Co-channel interference is a primary impediment in frequency reuse system. The goal of this chapter, is to propose a co-channel interference model for emerging and future cellular wireless communications system. Emerging and future cellular wireless systems are expected to operate at a carrier frequency higher than $2 \mathrm{GHz}$ and smaller cell size radius less than $1000 \mathrm{~m}$. The proposed co-channel interference model, includes both the first and second tier co-channel interfering cells. The proposed interference model is used for information capacity performance analysis for emerging and future cellular wireless systems in subsequent chapters of the thesis. The geometrical derivation shows that the number of co-channel interfering cells in a specific tier is the product of the tier number and the number of co-channel interfering cells in the first tier.

The cellular system was the initial philosopher's stone of wireless communication, called upon to transform wireless development into reality. Initially, the total number of radio channels offered to a land mobile system was not enough. Therefore, a satisfactory service was not provided within a metropolitan area on a large coverage area basis. However, the number of channels per unit area was increased by using the same radio channels in a small radio coverage area of cells within metropolitan areas. The same radio channels are separated sufficiently spatially, so that co-channel cells are at a reasonable distance to avoid co-channel interference, which reduces the information capacity performance of cellular systems [78].

In order to ensure that co-channel cells are reasonably spaced to avoid co-channel interference problem, cellular site layout is an important step in the design, deployment and management of cellular wireless communication networks. However, for conventional cellular systems, which have large cell size, large basic path loss exponent and operates at lower carrier frequencies, only the first tier co-channel interfering cells in the cell site layout were considered in interference modelling. All other co-channel interfering cells in subsequent tiers were considered to be negligible. Emerging and future wireless networks are expected to operate at microwave carrier frequencies greater than $2 \mathrm{GHz}$, thus 
enabling smaller cell size radius and smaller path loss exponent [28]. At higher carrier frequencies and smaller cell size radius, second tier and other tiers of co-channel interference may become active [45]. Co-channel interference modelling and information capacity performance analysis is important for next generation cellular wireless systems, because of the expected use of higher carrier frequencies and smaller cell size radius [79]. There is a need therefore, to propose a co-channel interference model. The proposed co-channel interference model is used for information capacity performance analysis for emerging and future cellular wireless system, expected to operate at higher microwave carrier frequencies and smaller cell size radius. The proposed co-channel interference model includes the first and second tier co-channel interfering cells. To be able to propose the new co-channel interference model, there is a need to find the number of co-channel interfering cells in a given tier of a cellular site layout. In $[1,35,43,44,80]$, the number of co-channel cells in the first, second and in some cases the third tier were given for different tessellations of planar regions. However, there are no explanation, formula or proof for how the number of subsequent tier co-channel interfering cells were obtained.

We were therefore motivated to propose a formula for determining the number of co-channel interfering cells in subsequent tiers. The proposed formula enables us to derive the proposed interference model. The proposed interference model was then used for the information capacity performance analysis, and finding the minimum cell size for uplink information capacity increase in a cellular wireless system, in the subsequent chapter of the thesis. The main contribution in this chapter is summarized as follows:

- An inductive approach is used to generate a formula for calculating the number of co-channel interfering cells beyond the first tier. The derivation of the formula in this chapter motivates the inclusion of other tier co-channel interfering cells, (i) in the information capacity performance analysis of cellular wireless networks and (ii) finding a theoretical limit to cell size radius reduction in cellular wireless communication systems.

- A co-channel interference model, which includes both the first and second tier cochannel interfering cells is proposed. The proposed co-channel interference model is used for information capacity performance analysis, for emerging and future cellular 
wireless communication systems in subsequent chapters of the thesis.

The remainder of this chapter is organized as follows: in Chapter 3.2, we describe the different tessellation of planar regions, which can be used for two-dimensional site layout for smaller size cellular systems. A formula for calculating the number of cochannel interfering cells in a given tier of a cellular site layout, is produced by an inductive approach. Chapter 3.3, describes the general system models and outlines the basic assumptions used in modelling the proposed co-channel interference to take into account the effect of second tier co-channel interference on the information capacity performance of cellular wireless systems, operating at higher carrier frequencies and smaller cell radii. In Chapter 3.4, a carrier-to-interference ratio, $C I R$ is worked out using path loss formula for LOS environment. Finally in Chapter 3.5, a summary of the chapter is presented. A portion of the work presented in this chapter is published in $[45,51]$.

\subsection{Frequency Reuse for Spectrum Allocation}

The cellular system is partly used to achieve spectrum efficiency by reusing frequencies at spatially-separated cells. In planning cellular systems, the whole service area is divided into non-overlapping cells. The non-overlapping cells cover the service area without gaps and each cell is covered by a base station site. A base station site in the center of a cell is depicted for a hexagonal site layout, in Figure 3.1. The distribution of frequency channels in a cellular wireless system depends on parameters, such as signal interference, signal propagation characteristics and cellular geometry. The cells are allocated a subset of the available radio spectrum such that the same frequency cells are sufficiently spatially separated. Thus, the spectrum is divided into $K$ disjoint subsets, and each cell is given among these subsets. Now if $K$ is one of the numbers $\{1,3,4,7,9,12,13,16,19,21, \ldots\}$, then the spectrum can be distributed in such a manner that for a hexagonal site layout every cell has a first tier of six co-channel interfering cells (cells using the same channel set).

A set of convenient co-ordinate systems was introduced by Mac Donald in 1979, for a hexagonal geometrical cell site layout [4]. Figure 3.2, illustrates the convenient coordinate system and it shows that the positive halves of the two axes intersect at a 60-degree angle. The unit distance along either axis is given by the product of $\sqrt{3}$ and the 


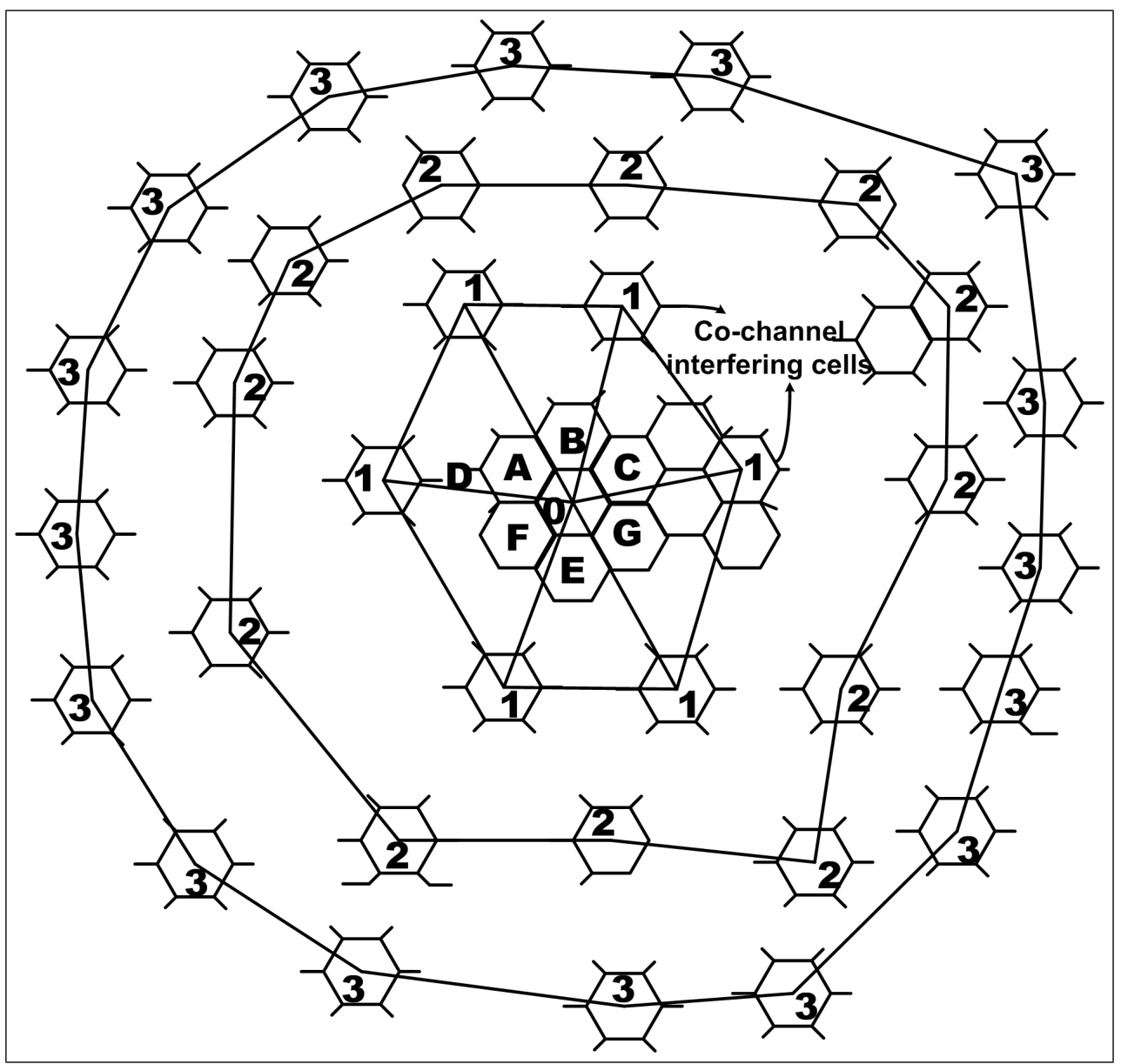

Figure 3.1: Cellular wireless system site coverage layout for a hexagonal tessellation.

1. 0 : coverage area under consideration (reference cell)

2. 1 : first tier co-channel interfering cells surrounding the reference cell

3. 2 : second tier co-channel interfering cells surrounding the reference cell

4. 3 : third tier co-channel interfering cells surrounding the reference cell

5. D : distance between cell centers

6. $A, B, C, E, F, G$ : coverage areas using frequencies different from the reference cell 


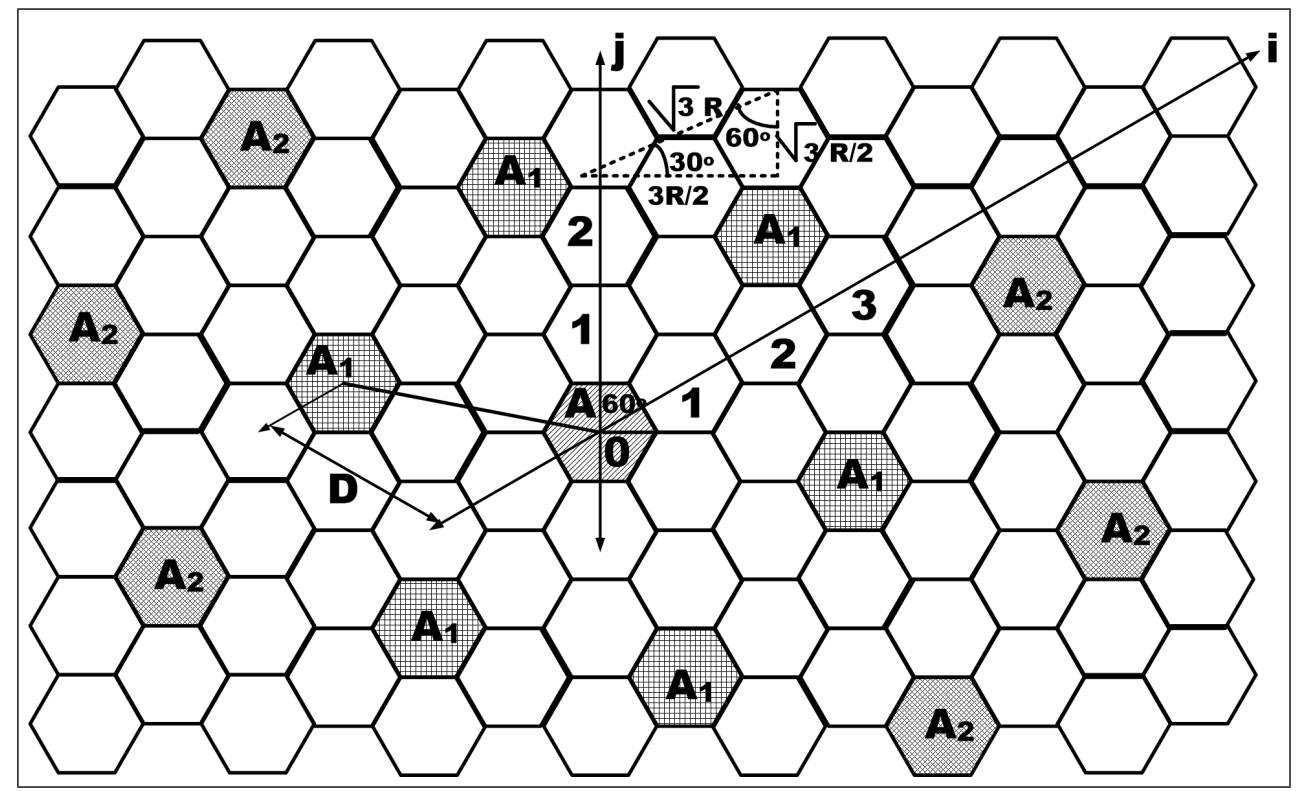

Figure 3.2: Co-ordinates for hexagonal geometry site layout in a cellular wireless system

cell size radius $R$ ( $\sqrt{3} R$ ), where $R$ corresponds to the distance from the center of a cell to any of its vertices. The coordinates of an array of cells are arranged such that the center of every cell falls at a point specified by a pair of integer coordinates. The reuse distance $D$ between the origin (A), to any cell center $\left(\mathrm{A}_{1}\right)$ is given by:

$$
D=\sqrt{i^{2}+i j+j^{2}}
$$

where $i$ and $j$ are positive integers.

\subsubsection{Cellular Wireless System Site Layout}

Cellular site layout patterns are usually represented by either equilateral triangles, squares or regular hexagons as they are the only regular polygons that tessellate in planar region [1]. In the derivation of the formula, for calculating the number of co-channel interfering cells, in this chapter all the three cellular site layout patterns are assumed. Reusing frequencies at spatially-separated cells in a cellular system introduces intercell interference. Intercell interference reduces the information capacity of all users. Thus desired users receive interference from co-channel cells as shown in Figure 3.1. In Figure 3.1, a desired user is located in the reference cell, 0 , and interference from the first tier are 


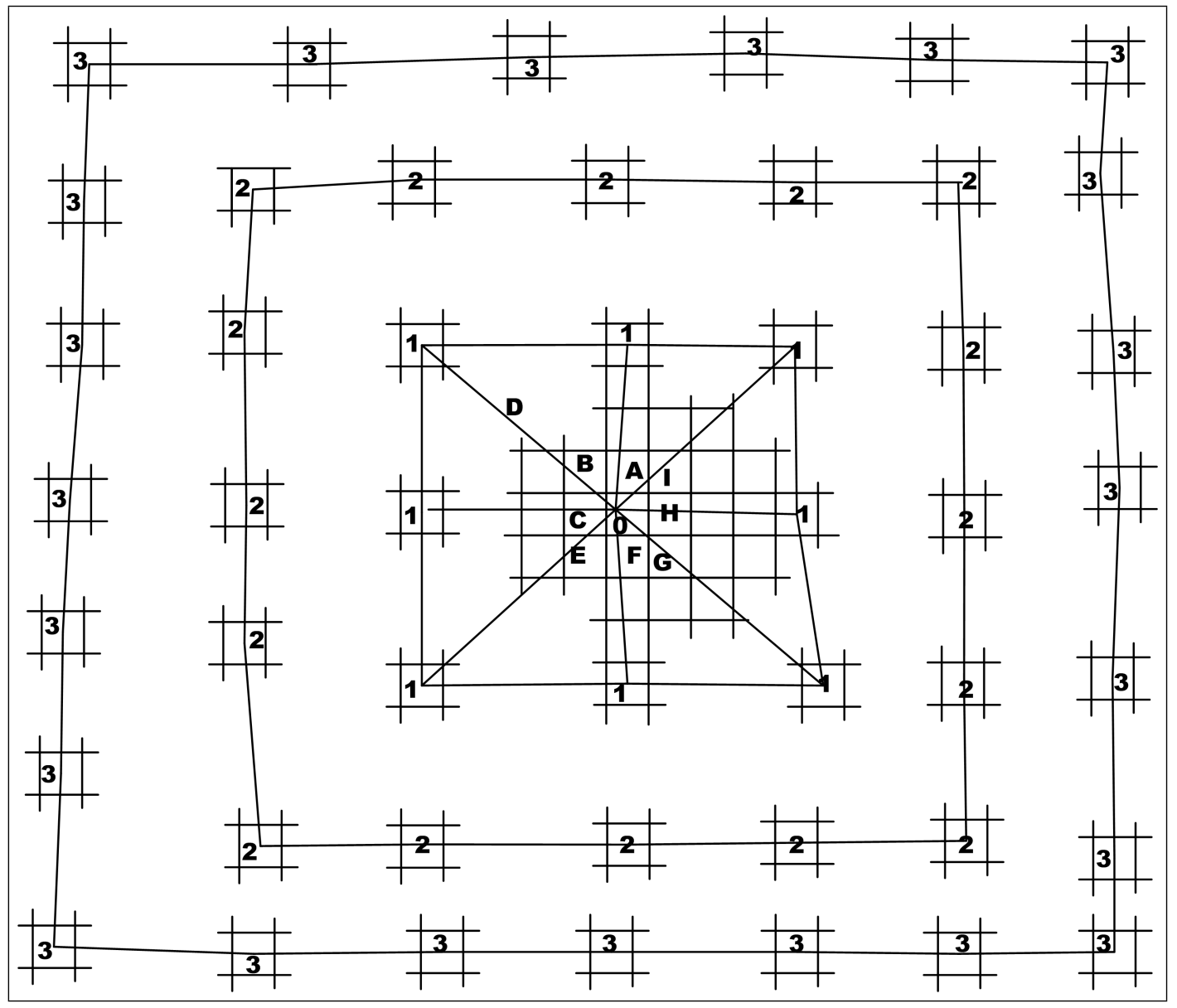

Figure 3.3: Cellular wireless system site coverage layout for a square tessellation.

1. 0 : coverage area under consideration (reference cell)

2. 1 : first tier co-channel interfering cells surrounding the reference cell

3. 2 : second tier co-channel interfering cells surrounding the reference cell

4. 3 : third tier co-channel interfering cells surrounding the reference cell

5. D : distance between cell centers

6. $A, B, C, E, F, G, H, I$ : coverage areas using frequencies different from the reference cell 


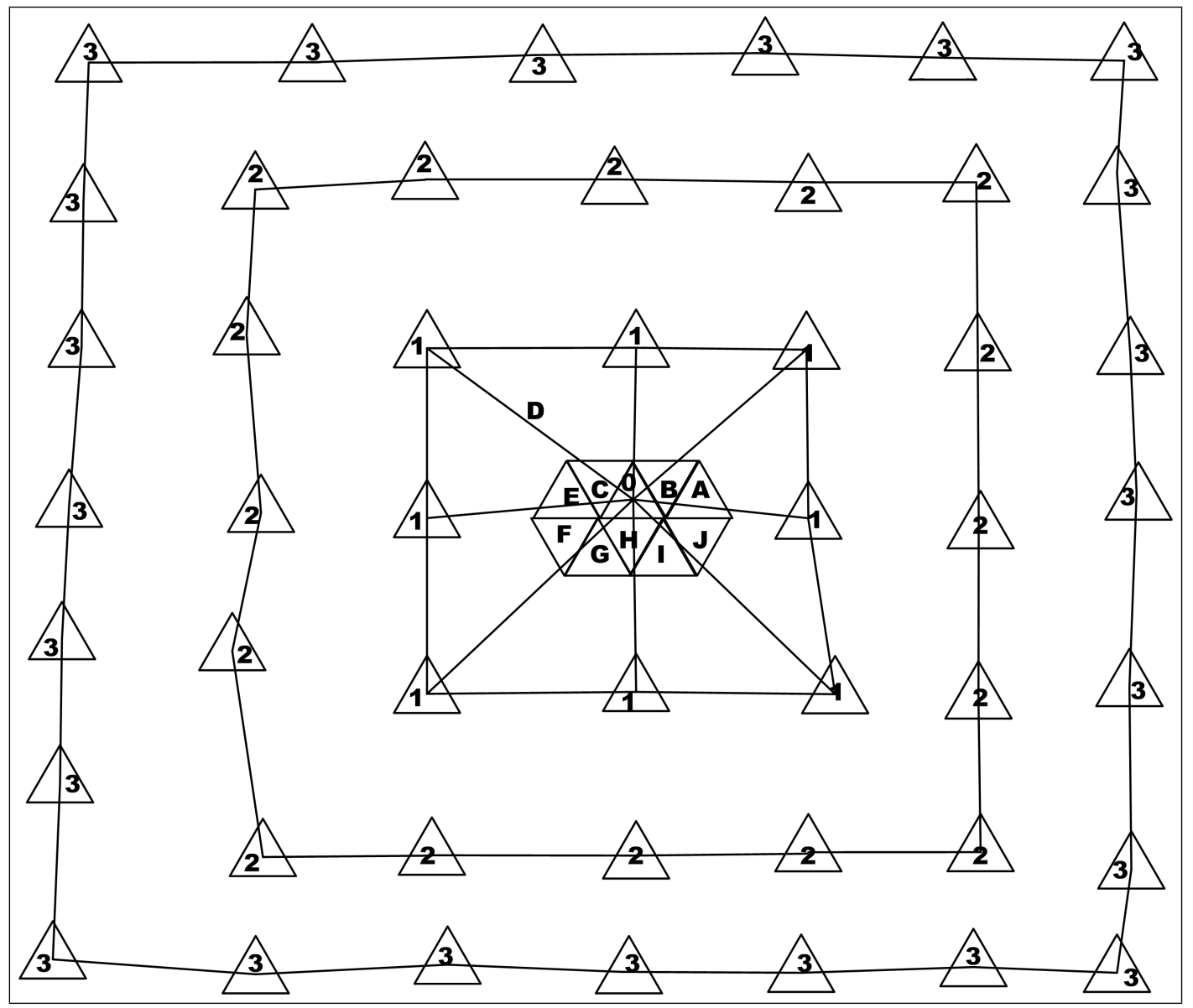

Figure 3.4: Cellular wireless system site coverage layout for a equilateral triangle tessellation.

1. 0 : coverage area under consideration (reference cell)

2. 1 : first tier co-channel interfering cells surrounding the reference cell

3. 2 : second tier co-channel interfering cells surrounding the reference cell

4. 3 : third tier co-channel interfering cells surrounding the reference cell

5. D : distance between cell centers

6. A,B,C,E,F,G,H,I,J : coverage areas using frequencies different from the reference cell 


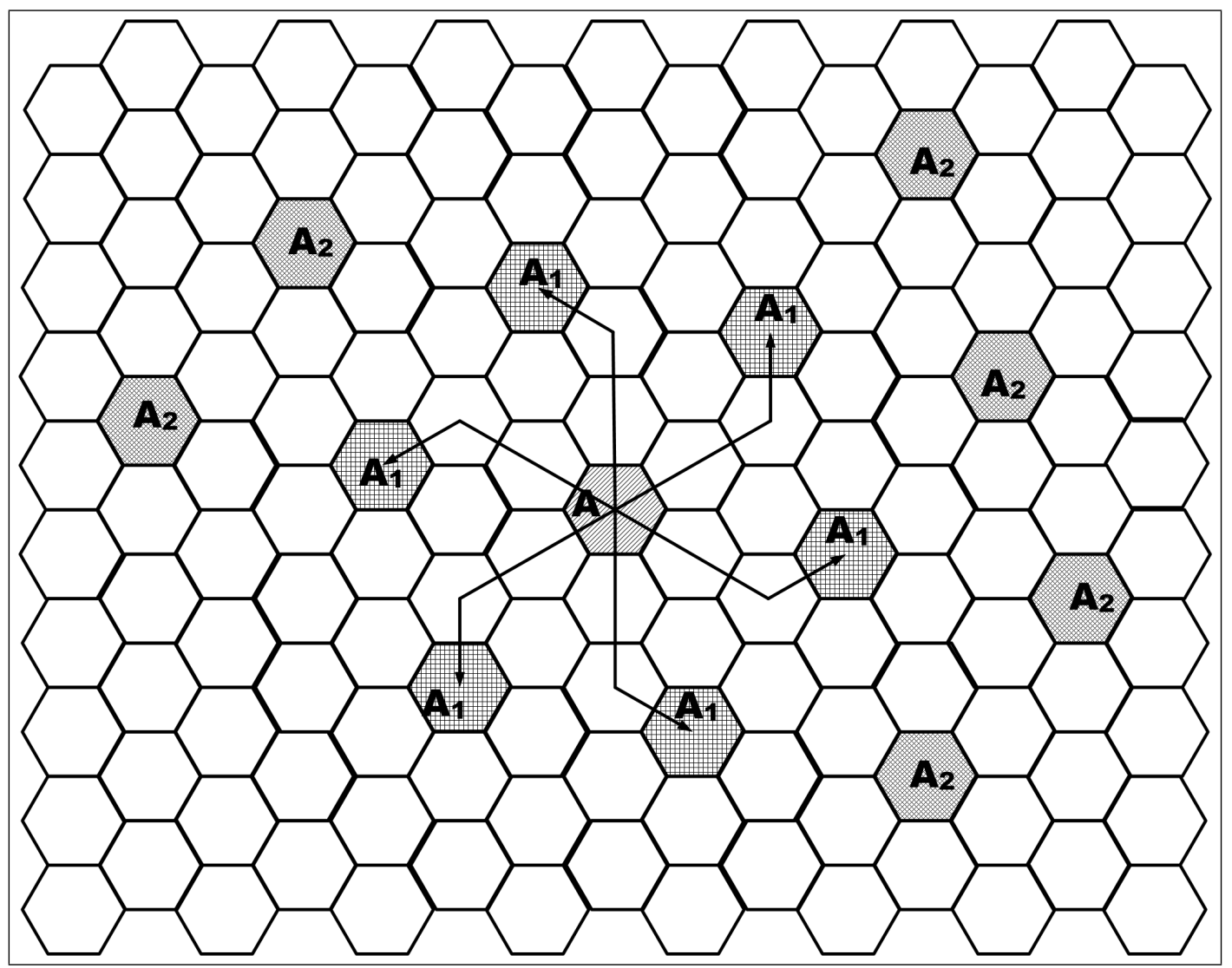

Figure 3.5: Illustration how "shift parameters" $(i, j)$ are used to lay out a cellular system. Shift parameters : $\mathrm{i}=2, \mathrm{j}=1$.

1. A : the reference cell

2. $\mathrm{A}_{1}$ : the first tier co-channel interfering cells

3. $\mathrm{A}_{2}$ : the second tier co-channel interfering cells

located in cell number 1, and second tier interference in cell number 2. The Figure 3.1 shows a hexagonal cell site layout tessellation. Figure 3.3, depicts that of a square site layout tessellation and Figure 3.4, illustrates that of equilateral triangle cellular site layout tessellation.

Figure 3.5 illustrates that for a hexagonal cell site layout tessellation, any cell has exactly six equidistant nearest neighbouring co-channel cells. The vectors from a cell center to the centers of its co-channel cells are separated by an angle of 60 degrees from each other, the same also holds for any arbitrary cell and its immediate six co-channel cell. Therefore, a cluster of contiguous cells can be visualised as a large hexagon. Note it is 
not claimed that all kinds of clusters will have a hexagon shape, but a large hexagon can have the same area as any valid cluster. From Figure 3.2, as the distance between centers of adjacent cells is unity, the distance between the centres of the visualised large hexagon is $\sqrt{i^{2}+i j+j^{2}}$. The pattern of the visualised large hexagons is just an enlarged replica of the original cellular pattern with a linear scale factor $\sqrt{i^{2}+i j+j^{2}}$. Thus, $N_{c}$, which is the cluster size and is the total number of cell areas contained in the area of the large hexagon, is given by the square of the linear scale factor as

$$
N_{c}=i^{2}+i j+j^{2} ; i, j \geq 0
$$

$i, j$, and the cluster size can only take certain realizable values. The realizable values range over the positive integers $(1,3,4,7,9,12, \ldots)$. For small cell systems design with square tessellation, to cover the whole service area in a symmetric cell plan the cluster size is given by [1] as

$$
N_{c}=i^{2}+j^{2} ; i, j \geq 0,
$$

As seen, with $i$ and $j$ being integers, the cluster size can only take certain realizable values which range over the positive integers $(1,2,4,5,8,9, \ldots)$.

The relationship between the normalized co-channel reuse distance $(D / R)$ and the number of cells per cluster in a hexagonal cell site layout can be found be combining the equation 3.1 and 3.2 and replacing the unity by $\sqrt{3}$ which gives us

$$
\frac{D}{R}=\sqrt{3 N_{c}}
$$

where $D$ (co-channel reuse distance), is the measured distance between the cell centers, $R$ is the cell size radius and $N_{c}$ is the cluster size.

Now after the number of cell per cluster is defined it is appropriate to find which channel set should be assigned to each cell. The frequency reuse layout of the cellular wireless communication system is easily assembled using the hexagonal coordinates of Figure 3.2, where $i$ and $j$ are called "shift parameters". The procedure for finding the nearest co-channel cells of any of the cells of the network is as follows:

- Choose a reference cell 
- For each side of the hexagon: move $i$ cells along any chain of hexagons then turn counterclockwise 60 degree, and move $j$ cells along the chain that lies on this new heading.

- Repeat the procedure for the surrounding cells of the initial reference cell which are not found as co-channel cells

Figure 3.5, shows how the "shift parameter" can be used to lay out a hexagonal cellular system site layout. The co-channel cells in first tier are labeled as $A_{1}$ and those in second tier are labeled $A_{2}$. The same set of channels used in cell $A$ are used in cells $A_{1}$ and $A_{2}$.

\subsubsection{Proposed formula for Calculating Number of Co-channel Cells}

In this section, the proposed formula for calculating the number of co-channel interfering cells is all tiers of a given cellular wireless site layout is generated. Figure 3.6, illustrates the geometry of a hexagonal cellular site layout with reuse pattern $\left(N_{c}=4\right)$. Figure 3.7, illustrates the geometry of a hexagonal cellular site layout with reuse pattern $\left(N_{c}=7\right)$. Figure 3.8, illustrates the geometry of a square cellular site layout with reuse pattern $\left(N_{c}\right.$ $=2$ ). Figure 3.9, illustrates the geometry of a equilateral triangle cellular site layout with reuse pattern $\left(N_{c}=2\right)$.

Considering the geometries $A B C D, E F G H$, IJKL, and $A B C D E F$, GHIJKL, MNOPQR in Figures 3.6 - 3.9, the geometry of the hexagonal cellular pattern with $N_{c}=4$ in Figure 3.6, shows that the co-channel cells in tier 1 are 8 , tier 2 are 16 and tier 3 are 24 . Likewise, the geometry of the hexagonal cellular pattern with $N_{c}=7$ in Figure 3.7, shows that the co-channel cells in tier 1 is 6 , tier 2 is 12 and tier 3 is 18 . The geometry of the square cellular pattern with $N_{c}=2$ in Figure 3.8, shows that the co-channel interfering cells in tier 1 is 8 , tier 2 is 16 and tier 3 is 24 . Finally, Figure 3.8, shows that for the equilateral triangle cell pattern with $N_{c}=2$, the number of co-channel interfering cells in tier 1 is 8 , tier 2 is 16 and tier 3 is 24 .

With the help of geometries $A B C D, E F G H, I J K L, A B C D E F, G H I J K L$ and $M N O P Q R$ in Figures 3.6 - 3.9, is shown that for a given cell site layout the number of co-channel interfering cells $N_{n}$ in the nth tier of the cellular site layout is the product of the tier number, 


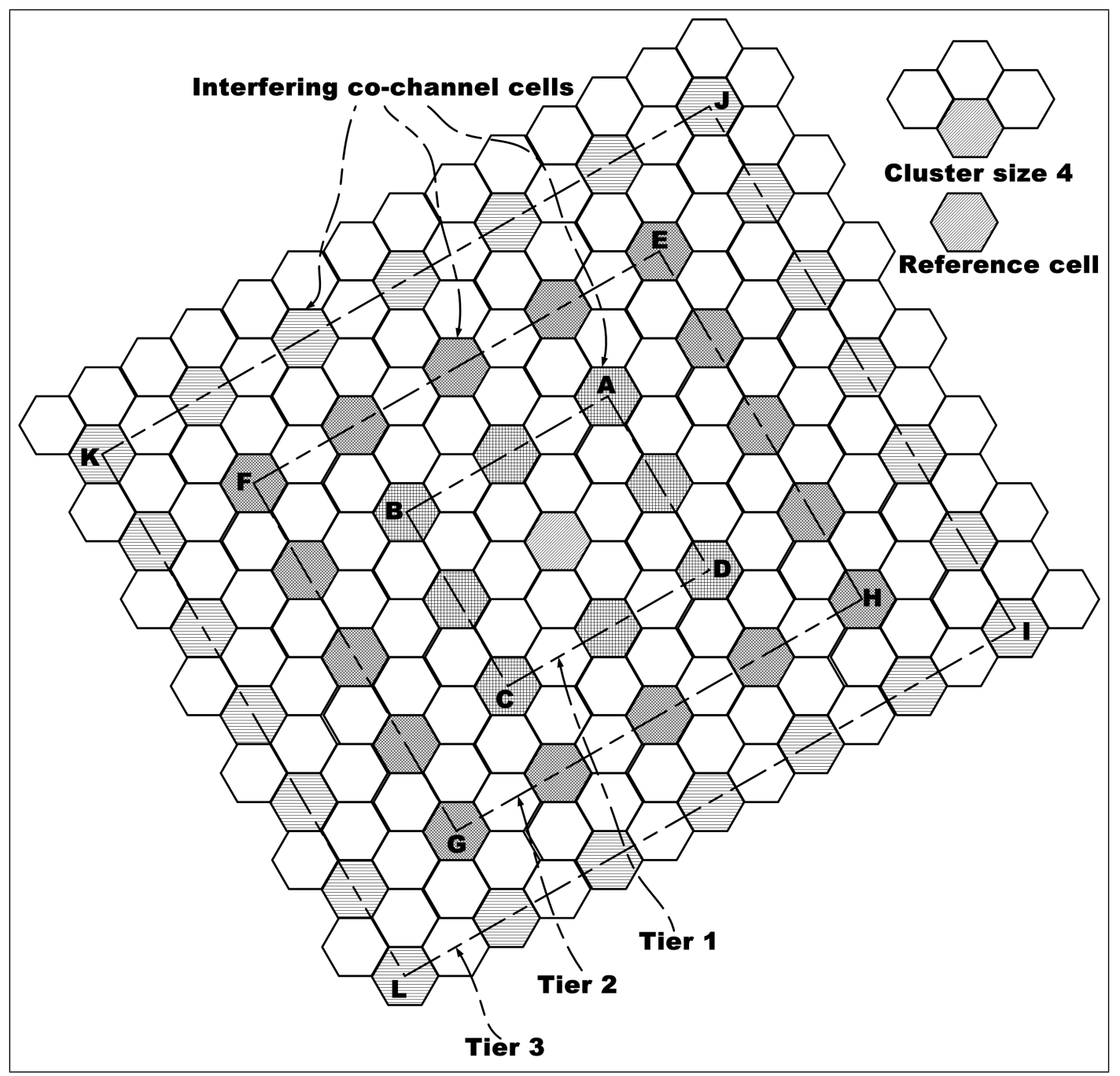

Figure 3.6: Geometry of a four cluster size regular hexagonal cellular system site layout.

1. Cells $A, B, C, D$ of square $A B C D$ represent first tier co-channel interfering cells

2. Cells $\mathrm{E}, \mathrm{F}, \mathrm{G}, \mathrm{H}$ of square $\mathrm{EFGH}$ represent second tier co-channel interfering cells

3. Cells I,J,K,L of square IJKL represent third tier co-channel interfering cells 


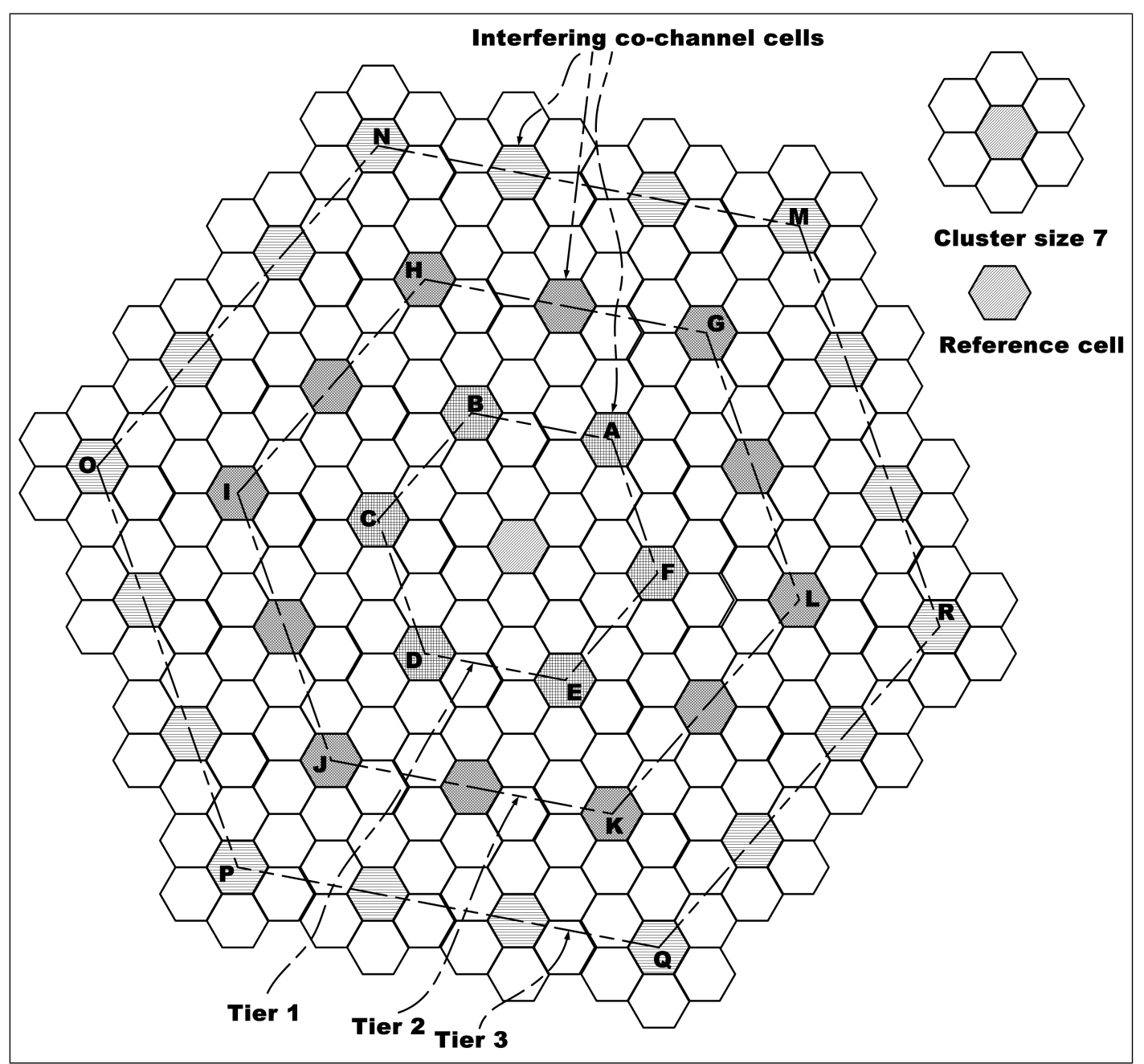

Figure 3.7: Geometry of a seven cluster size regular hexagonal cellular system site layout.

1. Cells $A, B, C, D, E, F$ of hexagonal $A B C D E F$ represent first tier co-channel interfering cells

2. Cells $\mathrm{G}, \mathrm{H}, \mathrm{I}, \mathrm{J}, \mathrm{K}, \mathrm{L}$ of hexagonal GHIJKL represent second tier co-channel interfering cells

3. Cells $\mathrm{M}, \mathrm{N}, \mathrm{O}, \mathrm{P}, \mathrm{Q}, \mathrm{R}$ of hexagonal MNOPQR represent third tier co-channel interfering cells 


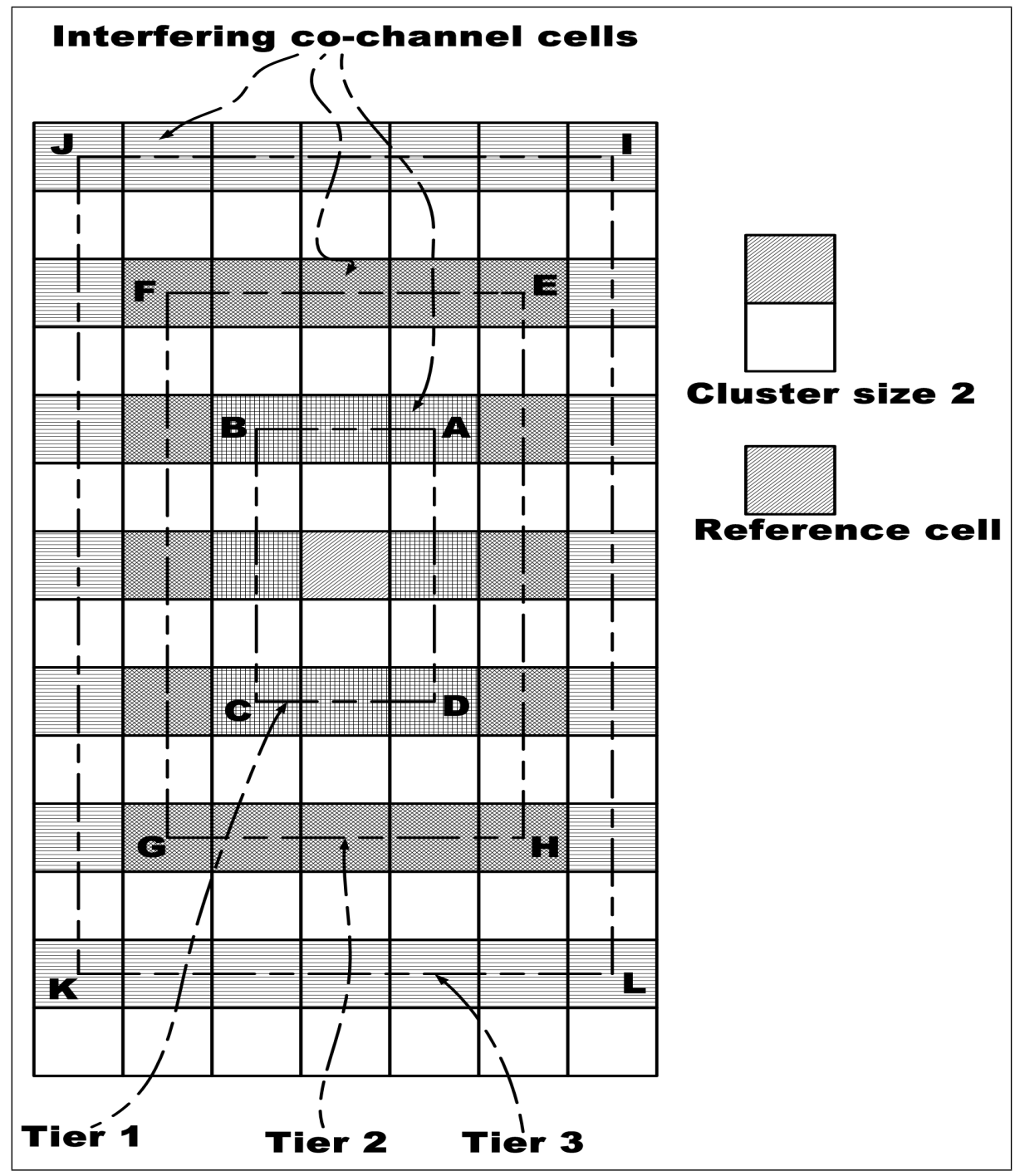

Figure 3.8: Geometry of a two cluster size square cellular system site layout.

1. Cells $A, B, C, D$ of square $A B C D$ represent first tier co-channel interfering cells

2. Cells E,F,G,H of square EFGH represent second tier co-channel interfering cells

3. Cells I,J,K,L of square IJKL represent third tier co-channel interfering cells 


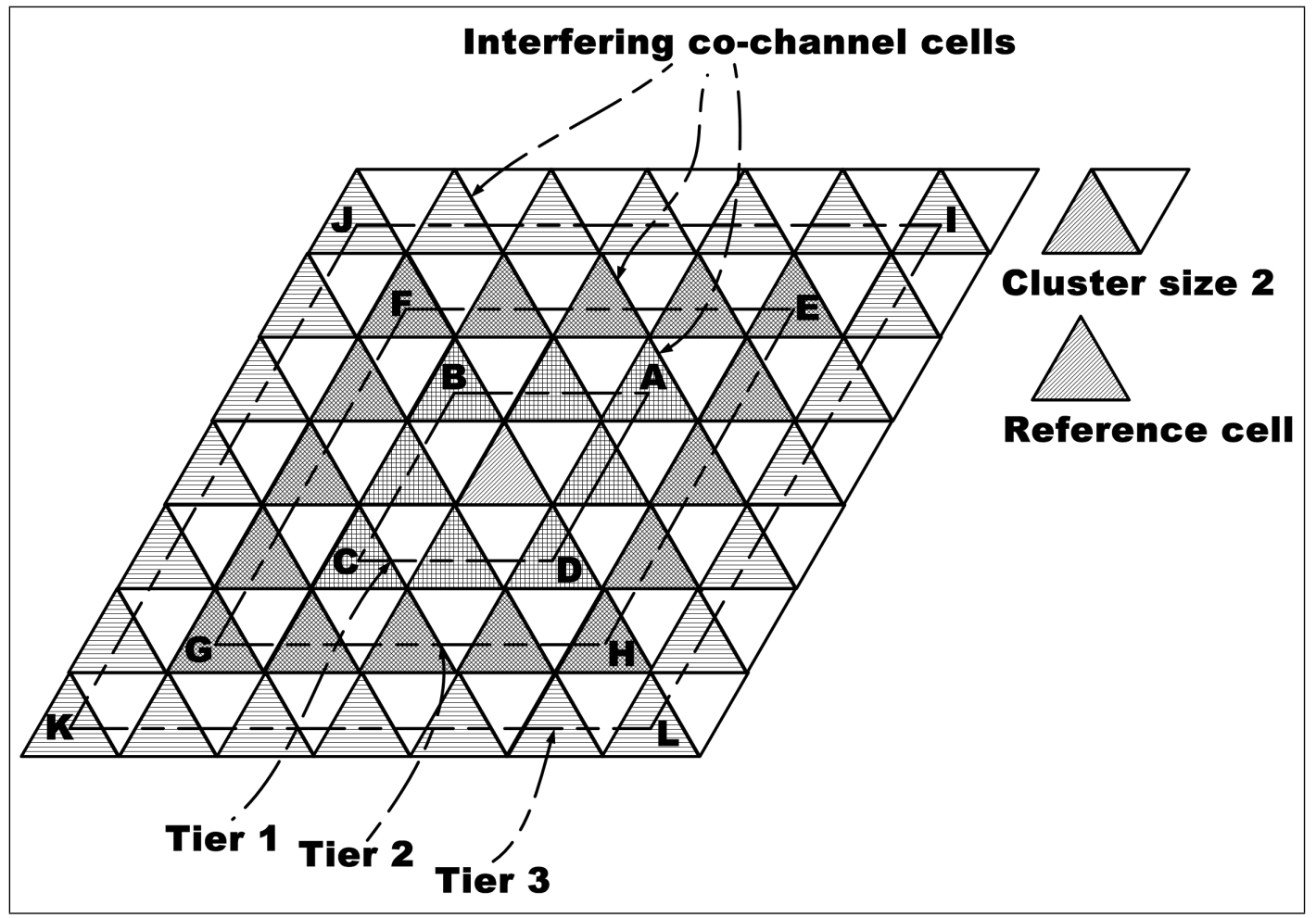

Figure 3.9: Geometry of a four cluster size equilateral triangle cellular system site layout.

1. Cells $A, B, C, D$ of square $A B C D$ represent first tier co-channel interfering cells

2. Cells E,F,G,H of square EFGH represent second tier co-channel interfering cells

3. Cells I,J,K,L of square IJKL represent third tier co-channel interfering cells 
and number of co-channels cells in the first tier, and can be written as:

$$
N_{n}=N_{F I} \times n ;(n=1,2,3,4, \ldots) .
$$

where $N_{F I}$ is the number of co-channel interfering cells in the first tier and $n$ is the nth tier number.

\subsection{Signal and Interference Model for Emerging Cellular Wireless Networks}

Cellular wireless communication systems are designed to exploit power falloff with distance of signal propagation to reuse the same frequency channel at a spatially separated location. However, the recent progress of communication services, means future and emerging wireless systems need to operate at higher microwave carrier frequencies in order to increase data rate. At higher carrier frequencies, both the free path loss and diffraction loss increase in relation to increase in frequency [2]. The increase in free space path loss means cell size radius needs to be reduced [2]. For cellular wireless communication systems to provide high-data-rate services, emerging and future cellular systems are designed to operate at higher microwave carrier frequencies $\left(f_{c}>2 \mathrm{GHz}\right)$ and smaller cell size radius $(R<1 \mathrm{~km})$. For conventional cellular systems, which operate at lower carrier frequencies $\left(f_{c}<2 \mathrm{GHz}\right)$ and have bigger cell size radius $(R>1 \mathrm{~km})$, other tier co-channel interfering cell are assumed to be in the region where power falloff is inversely proportional to the fourth power of distance. Hence, other tiers of co-channel interference are considered to be dormant. As will be discussed and explained in the following sections, using higher carrier frequencies and smaller cell size radius, in cellular systems may lead to other tier co-channel interfering cells becoming active. That is, other tier co-channel interfering cells may be in the same region of propagation as the first tier, where power falloffs are inversely proportional to the square of distance. In higher carrier frequency and smaller cell size radius cellular systems co-channel interference is expected to represent the main information capacity performance limitation. In the following sections we therefore propose an interference model for emerging and future cellular wireless systems. This will 


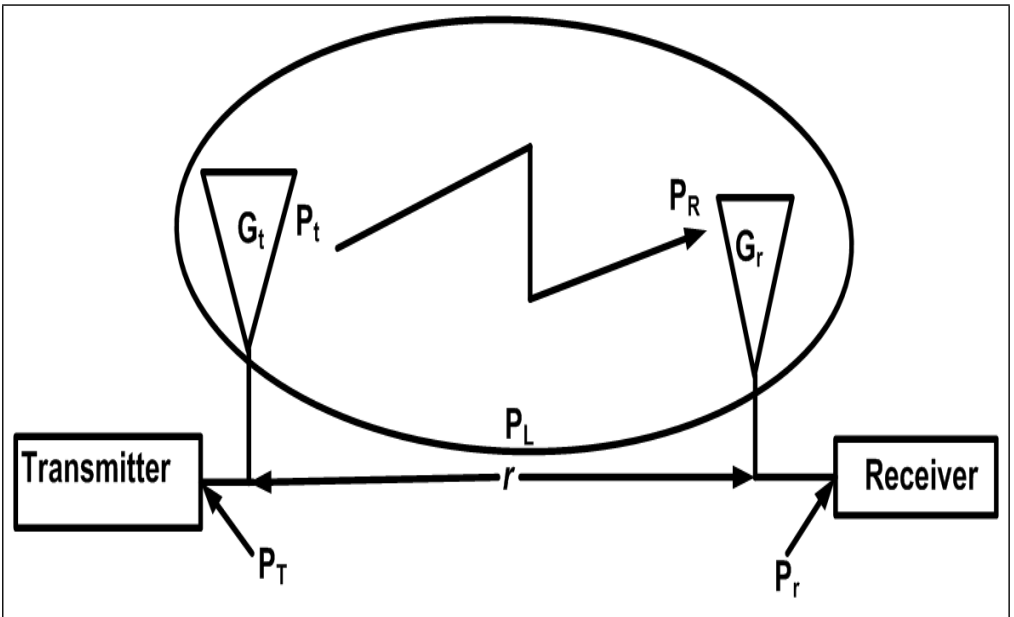

Figure 3.10: System propagation model for the uplink of a cellular wireless network: between MS and BS.

1. $\mathrm{P}_{T}=$ Transmit power

2. $\mathrm{P}_{t}=$ Transmitted signal power from antenna

3. $\mathrm{P}_{R}=$ Received power by antenna

4. $\mathrm{P}_{r}=$ Received signal power

5. $\mathrm{P}_{L}=$ Power-law path loss

6. $\mathrm{G}_{t}=$ Transmitter antenna gain

7. $\mathrm{G}_{r}=$ Receiver antenna gain

8. $r=$ Distance between transmitting and receiving antenna

enable accurate analysis of the information capacity performance of future and emerging cellular wireless communication systems.

\subsubsection{System Model for LOS Propagation Environment}

In a similar manner as in $[50,77]$, for both desired and interference signal power we consider a distance depended path loss wireless channel for our interference model. The LOS propagation system model is depicted in Figure 3.10. The distance depended path loss wireless channel used for our propagation system model and subsequent interference model in this thesis is valid. It is valid, because Sánchez et al. in their work in [81], 
reported that a 'snapshot' measurement may seriously underestimate the maximum mean electromagnetic field level that may be reached at a specific location. However, they also stated that 'snapshot' measurement results are still valid for information capacity performance analysis [81]. Therefore, in this thesis (i) for simplicity and (ii) due to the fact that it is a first order prediction analysis, we average out slow and fast fading, though fading may have considerable impact on the results. In this thesis for simplicity and ease of analysis, we assumed a cellular wireless communication system, which has an efficient antenna diversity scheme. The effective antenna diversity scheme eliminates the effects of multipath fading. Therefore, for a cellular wireless communication system having a base and mobile station antenna gain of unit, if a MS antenna transmitting a signal power of $P_{t}$ over a distance $r[\mathrm{~m}]$, the received signal power at the cell base station, $P_{r}[\mathrm{~W}]$ is given by [5] as

$$
P_{r}=\frac{K}{r^{\alpha}(1+r / g)^{\rho}} P_{t},
$$

where $K$ is the constant path loss factor, and it is the free space path loss at the reference distance $d_{0}=1 \mathrm{~m} . \alpha$, is the basic path loss exponent (roughly 2) and $\rho$ is the additional, (extra) path loss exponent (between 2-8). $g$ [m] is the breakpoint of the path loss curve. In this thesis the exact value of $K$ and $P_{t}$ is not required for the system model, because (i) all mobile users, (both those with the desired cell and out-of-cell interferers) transmit at the same signal power $P_{t}$ and (ii) the system is considered to be interference limited, therefore thermal noise is negligible. Therefore the exact value of $K$ and $P_{t}$ does not enter into the analysis [82]. Therefore, we assume $K=1, P_{t}=1$, and focus on the attenuation factor.

$$
P_{r}=r^{-\alpha}(1+r / g)^{-\rho}
$$

It must be noted that the path loss model, used for our system model in this thesis has been experimentally validated for a variety of microcellulr environments and for both UHF and SHF band [72, 83, 84].

\subsubsection{Relationship between Breakpoint Distance and Received Signal}

For conventional cellular wireless systems, the theoretical breakpoint distance is considered to be the product of the MS and BS antenna heights, and inversely proportional to 
Signal and Interference Model for Emerging Cellular Wireless Networks

\begin{tabular}{|l|l|l|l|l|}
\hline Carrier frequencies $\left(f_{c}\right)$ & $\lambda_{c}=\frac{3 * 10^{8}}{f_{c}}$ & $\mathrm{~h}_{m}$ & $g$ at $\mathrm{h}_{b}=15 \mathrm{~m}$ & $g$ at $\mathrm{h}_{b}=35 \mathrm{~m}$ \\
\hline $900 \mathrm{MHz}$ & $0.33 \mathrm{~m}$ & $1.5 \mathrm{~m}$ & $272.73 \mathrm{~m}$ & $636.36 \mathrm{~m}$ \\
\hline $2 \mathrm{GHz}$ & $0.15 \mathrm{~m}$ & $1.5 \mathrm{~m}$ & $600 \mathrm{~m}$ & $1400 \mathrm{~m}$ \\
\hline $3.35 \mathrm{GHz}$ & $0.09 \mathrm{~m}$ & $1.5 \mathrm{~m}$ & $1000 \mathrm{~m}$ & $2333.33 \mathrm{~m}$ \\
\hline $8.45 \mathrm{GHz}$ & $0.035 \mathrm{~m}$ & $1.5 \mathrm{~m}$ & $2571.43 \mathrm{~m}$ & $6000 \mathrm{~m}$ \\
\hline $15.75 \mathrm{GHz}$ & $0.019 \mathrm{~m}$ & $1.5 \mathrm{~m}$ & $4736.84 \mathrm{~m}$ & $11052.63 \mathrm{~m}$ \\
\hline
\end{tabular}

Table 3.1: [63]

the wavelength of the transmitter source. The theoretical breakpoint distance $g$ is given by

$$
g=\frac{4 \times h_{m} \times h_{b}}{\lambda} .
$$

where $h_{m}$ and $h_{b}$ are the MS and BS antenna heights, and $\lambda$ is the wavelength of the transmitting source. Table 3.1, presents a summary of theoretically calculated breakpoint distances for different carrier frequencies and BS antenna heights. Numerical values used for the calculation are from [85].

To make the relationship between the theoretical breakpoint distance, carrier frequency and received signal power explicable, using equation (3.6), a graph of received signal power $P_{r}[\mathrm{~W}]$ against distance $r[\mathrm{~m}]$ for different carrier frequencies $f_{c}[\mathrm{~Hz}]$ is plotted as shown in Figure 3.11. The breakpoint is indicated as $g$ on the graph. The plot in Figure 3.11, shows that for a distance dependent wireless channel two regions may be distinguished. The two regions are separated by the breakpoint. The region before the breakpoint is referred to as region I in this thesis. The region I, is where signal attenuation is assume to be inversely proportional to the second power of distance $r$ [m], between transmitter and receiver. The region after the breakpoint is referred to as region II. The region II, is where signal attenuation is assume to be inversely proportional to the fourth power of distance $r[\mathrm{~m}]$.

\section{Relationship between Breakpoint Distance and Carrier Frequency}

For the relationship between the theoretical breakpoint distance, carrier frequency and BS antenna height to be understandable, we plot a graph of breakpoint distance $g$ against 


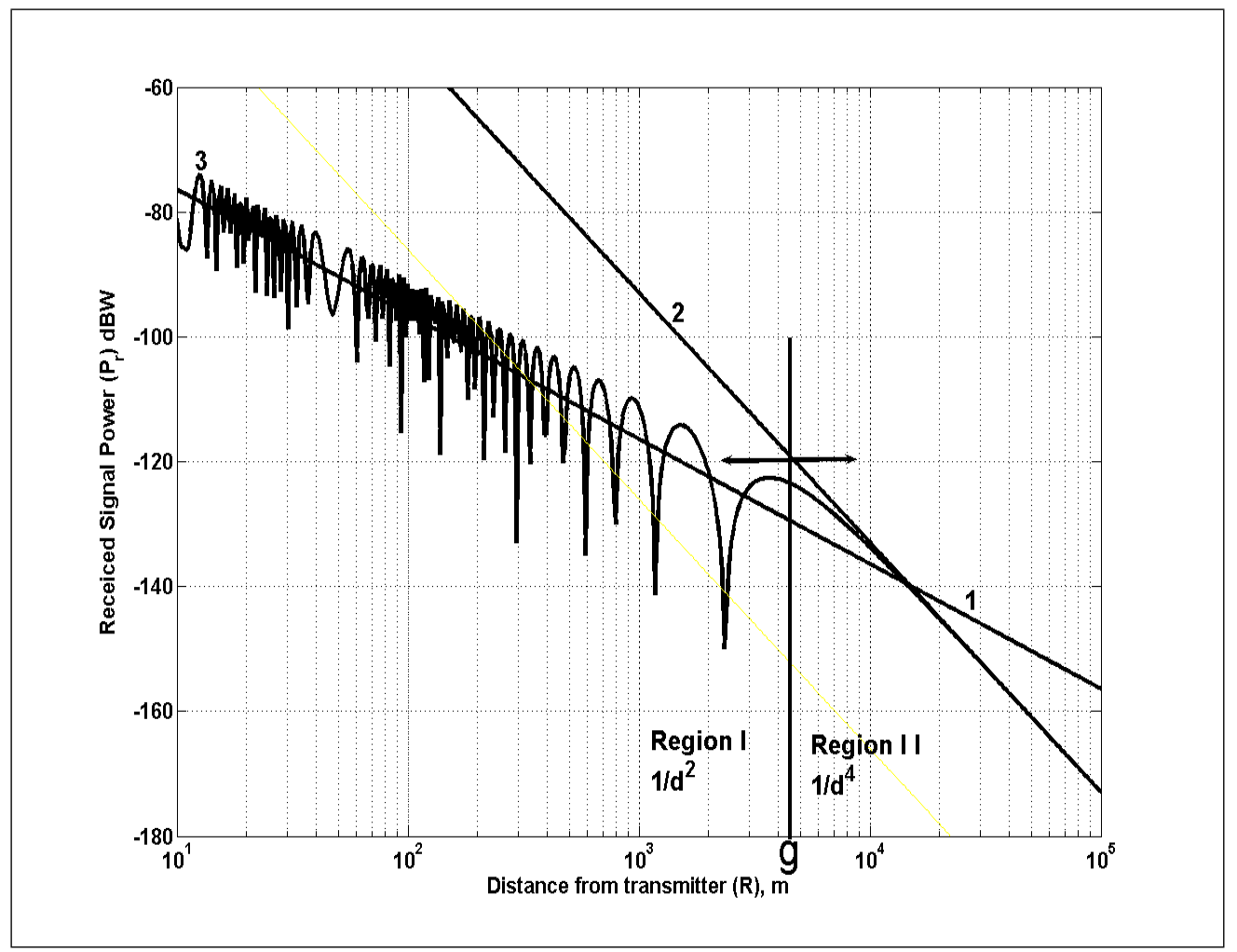

Figure 3.11: Two-slope received signal power, $P_{r}$ versus distance. Showing breakpoint distance $g$ at carrier frequency, $f_{c}=15.75 \mathrm{GHz}$. (Transmitted power $P_{t}=1 \mathrm{~W}$, MS and BS antenna gain $G_{r}=G_{t}=1$, basic path loss exponent $\alpha=2$, extra path loss exponent $\rho$ $=4$, MS and BS antenna heights: $h_{m}=1.5 \mathrm{~m}$ and $h_{b}=15 \mathrm{~m}$ )

1. Free Space Path Loss [86]

2. Power falls off $\propto 1 /(\text { distance })^{4}$

3. Two Ray path loss [68] 


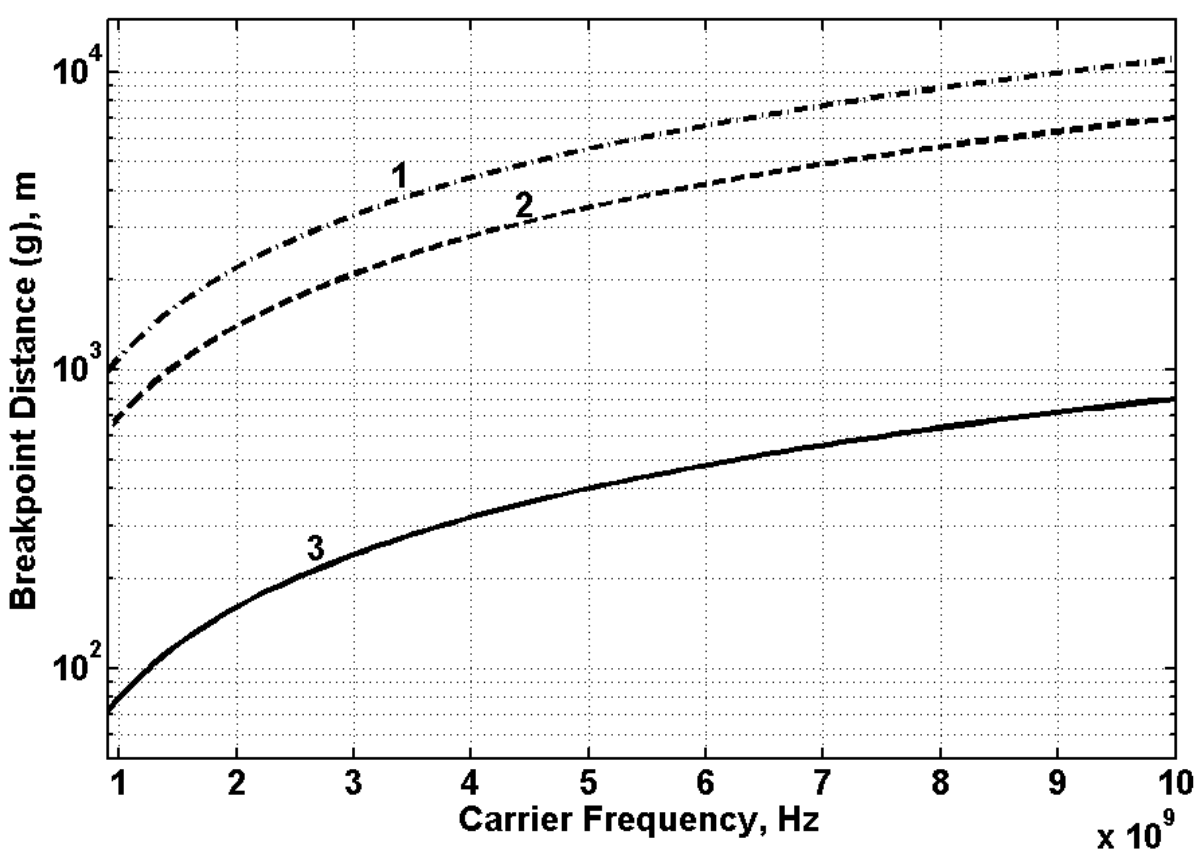

Figure 3.12: Breakpoint distance, $g$ versus carrier frequency, $f_{c}$. For MS antenna height, $h_{m}=1.5 \mathrm{~m}$, and different BS antenna height $h_{b}$.

1. BS antenna height, $\mathrm{h}_{b}=55 \mathrm{~m}$

2. BS antenna height, $\mathrm{h}_{b}=35 \mathrm{~m}$

3. BS antenna height, $\mathrm{h}_{b}=4 \mathrm{~m}$ 
Signal and Interference Model for Emerging Cellular Wireless Networks

\begin{tabular}{|l|l|l|l|l|l|}
\hline Carrier frequencies $\left(f_{c}\right)$ & $\lambda_{c}=\frac{3 * 10^{8}}{f_{c}}$ & $\mathrm{~h}_{m}$ & $\mathrm{~h}$ & $g_{h}$ at $\mathrm{h}_{b}=15 \mathrm{~m}$ & $g_{h}$ at $\mathrm{h}_{b}=35 \mathrm{~m}$ \\
\hline $3.35 \mathrm{GHz}$ & $0.09 \mathrm{~m}$ & $1.8 \mathrm{~m}$ & $0.3 \mathrm{~m}$ & $984.9 \mathrm{~m}$ & $2004.9 \mathrm{~m}$ \\
\hline $8.45 \mathrm{GHz}$ & $0.035 \mathrm{~m}$ & $1.8 \mathrm{~m}$ & $0.3 \mathrm{~m}$ & $2484.3 \mathrm{~m}$ & $5155.4 \mathrm{~m}$ \\
\hline $15.75 \mathrm{GHz}$ & $0.019 \mathrm{~m}$ & $1.8 \mathrm{~m}$ & $0.3 \mathrm{~m}$ & $46305 \mathrm{~m}$ & $9496.8 \mathrm{~m}$ \\
\hline
\end{tabular}

Table 3.2: [85]

carrier frequency $f_{c}$ for different BS antenna heights $h_{b}$, using equation (3.8). The plot in Figure 3.12, shows that for a given BS antenna height the breakpoint distance increases as the antenna height increases. The figure also shows that as the carrier frequency increases the breakpoint distance increases. As base station antenna height increases, carrier frequency increases, and the cell size radius decreases, second tier co-channel interference may become active. It may become active, because it is in the same region as the free space propagation region (region I), instead of the region II, as is assumed for conventional cellular wireless systems.

\subsubsection{Modified Breakpoint Distance for Higher Carrier Frequency}

For higher microwave carrier frequencies, the breakpoint distance is modified as described by Masui et al. in [85]. The modified breakpoint distance includes the effect of dispersion caused by reflection and blocking due to vehicles, pedestrians, and other objects on the road. The modified breakpoint distance for an effective road height, $h$ is given by [85] as

$$
r_{b r k}=\frac{4\left(h_{b}-h\right) \times\left(h_{m}-h\right)}{\lambda}, h<h_{m}
$$

The $h_{m}$ and $h_{b}$, are MS and BS antenna height. The effective road height, $h$ depends on the average road height, which is the average heights of vehicles and pedestrians on the road. For light vehicular traffic $h$, is between $0.23-0.74 \mathrm{~m}$. In the case of heavy vehicular traffic $h$, is between $1.29-1.64 \mathrm{~m}$ [87]. Table 3.2, presents a summary of the theoretical calculated modified breakpoint distances for light vehicular traffic conditions. Table 3.3 presents a summary of the theoretical calculated modified breakpoint distance for heavy vehicular traffic conditions. 
Signal and Interference Model for Emerging Cellular Wireless Networks

\begin{tabular}{|l|l|l|l|l|l|}
\hline Carrier frequencies $\left(f_{c}\right)$ & $\lambda_{c}=\frac{3 * 10^{8}}{f_{c}}$ & $\mathrm{~h}_{m}$ & $\mathrm{~h}$ & $g_{h}$ at $\mathrm{h}_{b}=15 \mathrm{~m}$ & $g_{h}$ at $\mathrm{h}_{b}=35 \mathrm{~m}$ \\
\hline $3.35 \mathrm{GHz}$ & $0.09 \mathrm{~m}$ & $1.8 \mathrm{~m}$ & $1.29 \mathrm{~m}$ & $312.314 \mathrm{~m}$ & $1947.7 \mathrm{~m}$ \\
\hline $8.45 \mathrm{GHz}$ & $0.035 \mathrm{~m}$ & $1.8 \mathrm{~m}$ & $1.29 \mathrm{~m}$ & $787.777 \mathrm{~m}$ & $5008.3 \mathrm{~m}$ \\
\hline $15.75 \mathrm{GHz}$ & $0.019 \mathrm{~m}$ & $1.8 \mathrm{~m}$ & $1.29 \mathrm{~m}$ & $1468.3 \mathrm{~m}$ & $9225.9 \mathrm{~m}$ \\
\hline
\end{tabular}

Table 3.3: [85]

\section{Relationship between Breakpoint Distance and Effective Road Height}

The relationship between the modified breakpoint distance, effective road height, $h$ and carrier frequency $f_{c}$ is made explicable, by plotting a graph of breakpoint distance against effective road height, for different carrier frequencies using equation (3.9). The plot in Figure 3.13, shows that the breakpoint distance tends to increase as the carrier frequency increases, but decreases as the effective road height increases.

\subsubsection{Cellular Wireless System Interference}

In cellular wireless communication systems the intercell interference magnitude depends on both the distance between the interfering transmitters and the intended receiver, as well as the propagation laws governing the interferer's transmission.

Generally the interference distribution is assumed to be Gaussian. This is a practicable assumption for CDMA systems, where there are many intracell and intercell interferes. The Gaussian distribution follows from the law of large numbers. However, for FDMA or TDMA systems, there are usually only a few active interferers. Therefore, the white noise assumption is generally not valid. For information capacity calculations in wireless systems, Gaussian interference is a worst-case noise assumption [57]. Under this assumption the information capacity-achieving transmit spectrum for all users (that is signal and interference), is Gaussian. In most cellular wireless communication systems the receiver noise power is much less than that of interference. Therefore, the receiver noise power can be neglected. In the generation of the proposed interference model we considered a TDMA cellular system, which has few active interferes. In the following sections, we first define the conventional cellular wireless co-channel interference model. 


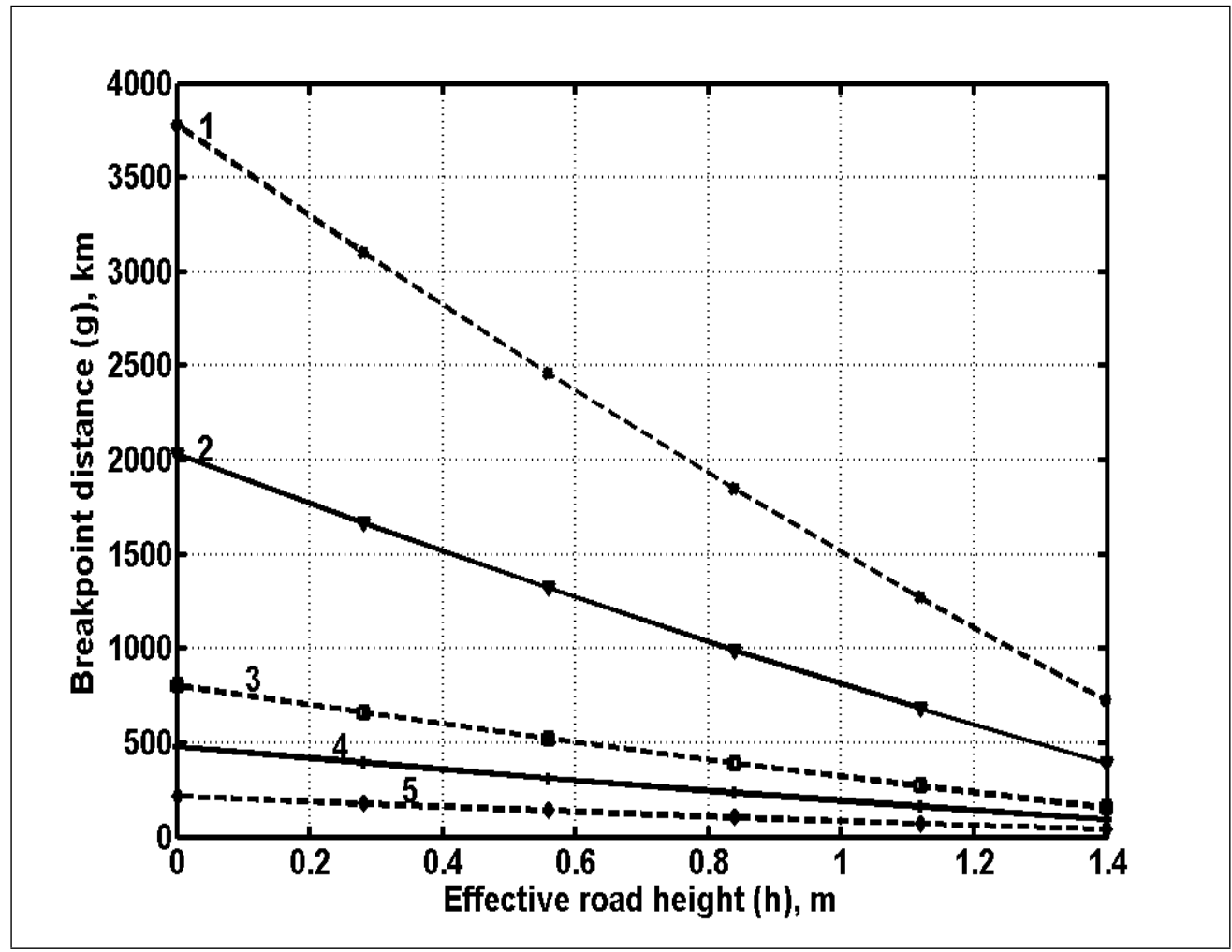

Figure 3.13: Breakpoint distance, $g$ vrs. effective road height, $h$ for different carrier frequency $f_{c}$. BS and MS antenna height; $h_{b}=10 \mathrm{~m}$ and $h_{m}=1.8 \mathrm{~m}$

1. Carrier frequency, $\left(f_{c}=15.75 \mathrm{GHz}\right)$

2. Carrier frequency, $\left(f_{c}=8.45 \mathrm{GHz}\right)$

3. Carrier frequency, $\left(f_{c}=3.35 \mathrm{GHz}\right)$

4. Carrier frequency, $\left(f_{c}=2 \mathrm{GHz}\right)$

5. Carrier frequency, $\left(f_{c}=900 \mathrm{MHz}\right)$ 
We then introduce our proposed co-channel interference model.

\subsubsection{Conventional Cellular Wireless Uplink Interference Model}

Co-channel interference can be considered to be a superposition of distance dependent attenuation (path loss), short-term fluctuations, and long-term variations. It occurs in wireless systems, for example, when a mobile simultaneously receives signals from co-channel BSs. For conventional cellular wireless networks co-channel interferences are assumed to be from the first tier only. This is based on the assumption of large path loss exponent, large cell size radius and lower carrier frequencies $\left(f_{c}<2 \mathrm{GHz}\right)$. For conventional cellular wireless systems the desired mobiles are assumed to be located in a central cell, and interfering mobiles to be in cells in the first tier as shown in Figure 1.6. In cellular wireless communication systems the uplink (from MS to BS) interference at a BS is the non-coherent sum of interference signals from the users served by the BS and the users served by other BSs [88]. The desired user $C I R, \gamma$, is defined as the ratio of the average received signal power from a MS at a distance $r[\mathrm{~m}]$ from the desired BS to the sum of interfering received signal power plus noise. Considering only intercell interference, for conventional cellular wireless communication systems the uplink $C I R, \gamma$ is given by [77] as

$$
\gamma_{d}=\frac{P_{d}}{P_{I}+N_{m}}=\frac{P_{d}(r)}{\sum_{i=1}^{N_{I}} P_{i}\left(r_{i}\right)+N_{m}}
$$

where $N_{m}$ is the thermal noise power, $P_{d}[\mathrm{~W}]$ is the received signal power level from the desired MS at a distance $r[\mathrm{~m}]$ from its BS. $P_{I}[\mathrm{~W}]$ is the total interfering power. $P_{i}$ [W] is the received signal power level from the ith interfering MS at a distance $r_{i}[\mathrm{~m}]$ from the desired mobile's BS. However, as stated in Chapter 2.3.1 additive noise term can be neglected relative to other channel impediments. Hence, for a conventional TDMA cellular wireless communication system the uplink $C I R, \gamma$ is written as

$$
\gamma_{d}=\frac{P_{d}}{P_{I}}=\frac{P_{d}(r)}{\sum_{i=1}^{N_{I}} P_{i}\left(r_{i}\right)}
$$




\subsection{Proposed Two Tier Uplink Interference Model}

For the initial proposed uplink interference model a two dimensional non-sectorized hexagonal cellular layout network is considered. The cellular site layout has six and twelve cochannel interfering cells in the first and second tier as illustrated in Figure 3.14. The BSs are assumed to be uniformly distributed, and cells form clusters (co-channel cells) around reference cells $\left(\mathrm{BS}_{0}\right)$. All BSs are located at the center of each cell and they receive signals from all the users (MSs) in the system. The signals received from all the users are attenuated according to the power-law path loss. All the BSs in the cells are fitted with omnidirectional antennas.

For the remainder of this section, we propose a two tier co-channel interference model for emerging and future cellular wireless communication systems. The proposed interference model, is used for the uplink information capacity performance analysis in the next chapter. In proposing the interference model we considered the first and second tiers of co-channel cell in our interference generation. The desired MS is considered to be located in the central cell and interfering mobiles are located in cells in the first and second tiers as shown in Figure 3.14. In Figure 3.14, $D$ denotes the distance between $B S_{0}$ and a first tier BS. A second tier BS is therefore, at a distance $2 D$ from $B S_{0}$. In order to simplify, and ensure accuracy of the analysis, each hexagonal cell is approximated as a circular cell with the equal area as in $[89,90]$. In cellular wireless communication, because uplink interference at a BS is the non-coherent sum of interference signals form the users served by the BS and the users served by other BSs [88], for a non-sectorized cellular system, the carrier-to-noise ratio $C N R$ can be written as follows

$$
\gamma_{d}=\frac{P_{d}}{P_{I}+N_{m}}=\frac{P_{d}(r)}{\sum_{f i=1}^{N_{F I}} P_{f i}\left(r_{f i}\right)+\sum_{s i=1}^{N_{S I}} P_{s i}\left(r_{s i}\right)+N_{m}} .
$$

where $N_{F I}$ is the number of interfering cells in the first tier. $N_{S I}$ is the number of cochannel interfering cells in the second tier. $N_{S I}$ is calculated using equation (3.5), for $N_{F I}$ $=6, N_{S I}=6 \times 2=12 . P_{d}$ is the received signal power of the desired MS. $P_{I}$, is the total interfering power from the first and second tier. $P_{f i}$ and $P_{s i}[\mathrm{~W}]$ are the average power level received from the ith interfering mobiles at distances $r_{f i}[\mathrm{~m}]$ and $r_{s i}[\mathrm{~m}]$ in the first and second tier from the desired BS. 


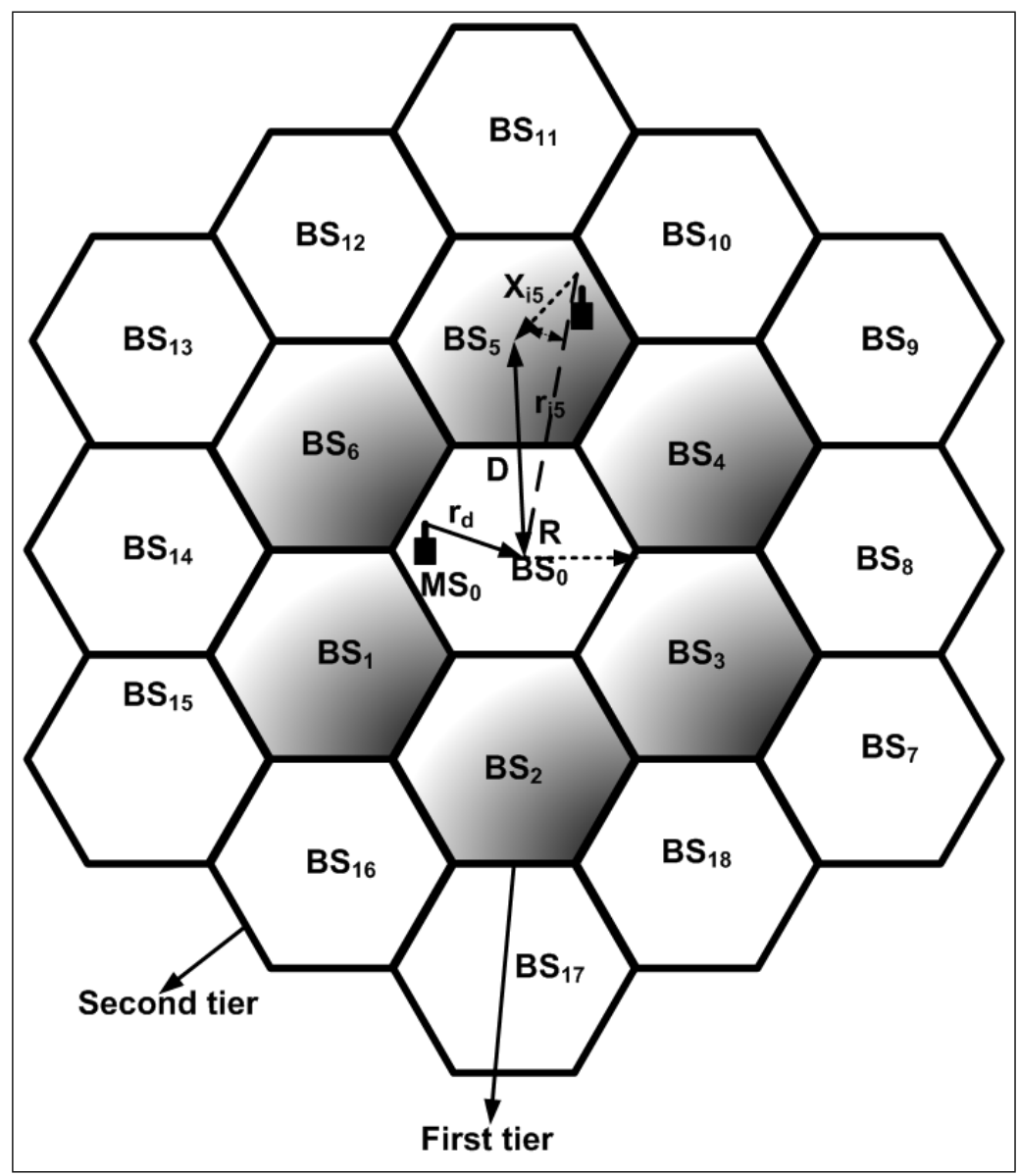

Figure 3.14: Uplink co-channel interference on a desired BS of a fully loaded nonsectorized hexagonal cellular system model, with 6 and 12 co-channel interfering cells in the first and second tier, cluster size $\mathrm{N}_{c}=7$.

1. $\mathrm{BS}_{0}$ : the reference cell

2. $\mathrm{MS}_{0}$ : the desired MS

3. $\mathrm{R}$ : the cell radius

4. $\mathrm{r}_{d}$ : the distance of separation between $\mathrm{MS}_{0}$ and $\mathrm{BS}_{0}$

5. $\mathrm{D}$ : the distance of separation between $\mathrm{BS}_{0}$ and $\mathrm{BS}_{5}$

6. $\mathrm{D}_{1}$ : the distance of separation between $\mathrm{BS}_{0}$ and $\mathrm{BS}_{7}$

7. $\mathrm{B}_{1-6}$ : the first tier co-channel interfering cells

8. $\mathrm{B}_{7-12}$ : the second tier co-channel interfering cells 
To simplify our analysis we made the following assumptions in the co-channel interference model. First the system is considered to be interference-limited, with thermal noise power negligible relative to the co-channel interference power [61]. Therefore, carrier to noise ratio $C N R$ reduces to the carrier-to-interference power ratio $C I R$. All inter-channel interferences are considered to be negligible [61]. All MSs are assumed to transmit the same power, and for simplicity we assume each cell to be circular in shape. Throughout the thesis it is assumed that co-channel interfering signals add up incoherently as this leads to a more realistic assessment of co-channel interference in cellular wireless network as reported in [91][92].

Considering a non-sectorized, and interference limited cellular wireless communication system with $N_{F I}$, and $N_{S I}$ co-channel interfering cells in the first and second tier, the desired user $C I R, \gamma_{d}$ is the ratio of average received power level $P_{d}$, of desired mobile to the total interfering power $P_{I} . P_{I}$ is the power sum of individual interferers in the first tier plus the power sum of individual interferers in second tier. Therefore $C I R$ can be written as:

$$
\gamma_{d}=\frac{P_{d}}{P_{I}}=\frac{P_{d}(r)}{\sum_{f i=1}^{N_{F I}} P_{f i}\left(r_{f i}\right)+\sum_{s i=1}^{N_{S I}} P_{s i}\left(r_{s i}\right)} .
$$

where $P_{f i}$ and $P_{s i}[\mathrm{~W}]$ are the average power level received from the ith interfering mobiles at distances $r_{f i}[\mathrm{~m}]$ and $r_{s i}[\mathrm{~m}]$ in the first and second tier from the desired BS. Since the signal powers for both the desired and interfering mobiles experience fluctuation, because of the random location of MSs in their cells, $\gamma_{d}$ is also a random variable. $\gamma_{d}$ depends on the distribution of $P_{d}, P_{f i}$ 's and $P_{s i}$ 's, which in this thesis are assumed to be uniformly distributed.

\subsection{Summary}

In this chapter, we have studied cellular site layout. An inductive approach was used to generate formula for calculating the number of co-channel interfering cells in subsequent tiers of a cellular wireless system site layout. It has been shown that for the three tessellations of planar region used for cellular wireless communication cell site layout, the number of co-channel interfering cells in any tier is the product of the tier number and the number 
of co-channel interfering cells in the first tier. Next, an improved co-channel interference model has been proposed. Unlike the conventional, co-channel interference model in [77], the proposed interference model includes the second tier co-channel interfering cells.

In the greater most part of this chapter we have proposed a formula for calculating the number of co-channel interfering cells in cell site layout, and a co-channel interference model which includes the first and second tier co-channel interfering cells. These we will use in subsequent chapters for, (i) information capacity performance analysis, and (ii) finding the theoretical limit to cell size radius reduction for information capacity increase in cellular wireless systems operating at higher carrier frequencies. In the next chapter, we mathematically characterize the area spectral efficiency, $A S E$ as a function of the proposed co-channel interference model for information capacity performance analysis. Information capacity performance analysis is performed to find out if at higher microwave carrier frequencies and smaller cell size radius second tier co-channel interfering cells becomes active. This is achieved by comparing the $A S E$ which incorporates our proposed co-channel interference model with an existing $A S E$ (reference [77]), of a cellular mobile radio system. The next chapter studies the effect of system and propagation loss parameters on the information capacity performance of cellular wireless networks operating at higher carrier frequencies and smaller cell radius when both first and second tier co-channel interfering cells are active. 


\section{Chapter 4}

\section{Information Capacity Performance Analysis for Two Tier Co-channel Interference}




\subsection{Introduction}

In the previous chapter, we proposed a novel formula for calculating the number of cochannel interfering cells in subsequent tiers of a cellular wireless communication system site layout. A co-channel interference model which includes the second tier co-channel interfering cells was proposed. In this chapter, using conventional and the proposed cochannel interference model, an information capacity analysis for cellular wireless communication system is performed.

The information capacity performance analysis was accomplished by comparing the area spectral efficiency, which incorporates our proposed interference model, to the area spectral efficiency, that incorporates the conventional interference model [77]. The information capacity analysis shows that at carrier frequencies $f_{c}>2 \mathrm{GHz}(3.35-15.75$ $\mathrm{GHz}$ ), and cell size radius $R<500 \mathrm{~m}$, second tier co-channel interfering cells become active. Which is the bases for the decrease in the information capacity of the cellular wireless system. A study on the impact of system and propagation loss parameters (such as path loss exponent, effective road height, antenna height), on the information capacity of a cellular wireless system is then performed. Results, show that for both distance dependent, and effective road height, the information capacity performance of a cellular wireless communication system is sensitive to system and propagation loss parameters at carrier frequencies $f_{c}>2 \mathrm{GHz}$.

The propagation environment for wireless communications, has been one of the major sources of information capacity performance degradation. That is to say that propagation loss parameters affect the information capacity performance of cellular wireless communication systems. In wireless propagation environments there are different categories of environments such as buildings, trees, roads water and homes [93]. The wireless propagation environment therefore, requires an accurate modelling [52] for information capacity performance analysis of a cellular system. Therefore, it is necessary to study the information capacity performance of a cellular wireless network under different propagation loss parameters. System parameters also affect the information capacity performance of cellular wireless systems. It is therefore, important to study the impact of system parameters on the information capacity performance of emerging and future cellular systems. 
The impact of propagation loss and system parameters on the information capacity performance of land mobile cellular wireless networks has been studied and published in previous literature [28, 46, 47, 77, 94-101]. However for most of these studies to proceed analytically, only first tier co-channel interfering cells were incorporated into the interference model. It was assumed that interference beyond the first tier (subsequent tiers) was negligible. This assumption was based on large path loss exponent [7]. This was suitable for microwave carrier frequencies $f_{c}=0.9$ and $1.8 \mathrm{GHz}$ and cell size radius $R>1 \mathrm{~km}$, which was the case for conventional cellular wireless communication systems.

Cox et al. in [94], performed an experiment to show the dependence of wireless signal attenuation on antenna height. However, the carrier frequency used for the experiment was $815 \mathrm{MHz}$. Green in [95], carried out signal and path loss variability analysis for small cells and concluded that path loss characteristics within smaller cells consist of two distinct and separate regions (regions before and after the breakpoint $g$ ). The analysis was for carrier frequencies $f_{c}>2 \mathrm{GHz}$, but only first tier co-channel interfering cells were considered. Cruz-Pérez and Lara-Rodríguez in [96] studied the impact of breakpoint distance on the capacity of Manhattan like microcellular CDMA system. In the work of Cruz-Pérez and Lara-Rodríguez interference was assumed to be from the first tier, and they did not consider hexagonal cell site layout. In [97], Min and Bertoni studied the effect of path loss model on CDMA system design for highway microcells. However, they considered a highway scenario where interference came from the first tier.

Alouini and Goldsmith, studied the effect of normalized reuse distance, path loss and cell radius on the information capacity performance of cellular wireless communication system for carrier frequency $f_{c}=0.9$ and $2 \mathrm{GHz}$ [77]. Alouini and Goldsmith did not consider carrier frequencies $f_{c}>2 \mathrm{GHz}$, and effective road height. Har and Bertoni, studied the effect of cell parameters on microcell planning considering the first and second tier co-channel interfering cells, without considering carrier frequencies $f_{c}>2 \mathrm{GHz}[100]$. Hernández et al. studied the effects of cell size radius reduction and path loss model parameters on the performance of microcellular networks without second tier co-channel interfering cells [99]. In [28], Hernández et al. studied the sensitivity of system performance to propagation loss and system parameters in LOS microcellular environment for carrier frequencies $f_{c}>2 \mathrm{GHz}$. However, outage probability was used for the perfor- 
mance analysis. They considered only urban, and cigar-shaped microcellular environment cellular site layout.

The influence of trees on the radio channel at carrier frequencies of 3 and $5 \mathrm{GHz}$, was studied in [102]. Masui et al. in [85], proposed a new path loss model for the characterization of microwave urban LOS propagation. That is, introducing effective road height in cellular wireless communication system information capacity performance analysis. Anang et al. in $[46,47]$ studied the impact of base station antenna height and path loss exponent on the uplink information capacity of a cellular wireless system operating at carrier frequencies $f_{c}>2 \mathrm{GHz}$. In their study, second tier co-channel interfering cells were considered to be active. However, the effect of effective road height was not considered.

Emerging and future cellular wireless systems will be operating at microwave carrier frequency $f_{c}>2 \mathrm{GHz}$ and smaller cell size radii $R \leq 1 \mathrm{~km}$. From the previous chapter we have proposed an interference model which includes second tier co-channel interfering cells. It is therefore, necessary to provide information capacity performance comparison between the conventional and our proposed co-channel interfering model. In this chapter our contribution is as follows:

- This chapter provides an uplink information capacity performance comparison for cellular wireless communication system using our proposed and conventional interference model.

- To study the impact of propagation loss and system parameters (such as BS antenna height, effective road height) on the uplink information capacity of a cellular wireless system using the proposed interference model.

- The numerical result shows that at carrier frequencies $f_{c}>2 \mathrm{GHz}$ and smaller cell size radii $R \leq 500 \mathrm{~m}$ second tier co-channel interfering cell becomes active.

- The numerical result shows that cellular wireless communication system information capacity performance are sensitivity to system and propagation loss parameters, even when second tier co-channel interfering cell becomes active.

The reminder of this chapter is organized as follows. Chapter 4.2, describes the general 
system models and outlines the basic assumption for our system modelling. Chapter 4.3, presents the system model for information capacity performance analysis. The simulation model and simulation parameters used for the information capacity performance comparison are presented in Chapter 4.4. Chapter 4.5, provides numerical results for the information capacity performance comparison. Finally, a summary of the chapter is presented in Chapter 4.6. The work in this chapter is published in [46-49].

\subsection{Two - Tier Co-channel Interference Model}

For the information capacity performance comparison, a cellular wireless system operating at carrier frequencies $f_{c}>2 \mathrm{GHz}$, and having smaller cell size radius $R \leq 1 \mathrm{~km}$ is considered. The first and second tier co-channel interfering cells are assumed to be active as described in Chapter 3.3. Using the same notations in Chapter 3.4, the desired MS carrier-to-interference ratio $C I R, \gamma_{d}$ is mathematically represented by equation (3.13).

\subsubsection{Mobile and Interfering Users Distribution in Cells}

For mathematical convenience and accuracy of the analysis, the cell shape is approximated by a circle of radius $R$ and all cells are assumed to have equal area. It is assumed erroneously that all MS are within the circle of radius $R$. Desired MS's and interfering MS are assumed to be uniformly, and independently distributed, $[0,2 \pi]$ in their respective cells as illustrated in Figure 4.1. The uniform and independent distribution of MS was chosen (i) for its simplicity, and (ii) because it provides a general and realistic representation of real life cellular wireless communication systems [103]. Note, we also assumed MS are located in the far field region. Therefore, we describe the probability distribution function (PDF) of a mobile location relative to a $\mathrm{BS}$ as

$$
p_{r, \theta}(r, \theta)=\frac{\left(r-R_{0}\right)}{\pi\left(R-R_{0}\right)^{2}} ; R_{0} \leq r \leq R, 0 \leq \theta \leq 2 \pi .
$$

where $R_{0}$ represents the minimum distance a mobile can be from a BS antenna. It defines a small circular area around the MS to be kept free from interferes (that is, the far field region). A reasonable value around $20 \mathrm{~m}$ is normally recommended for smaller cell size cellular wireless systems. 


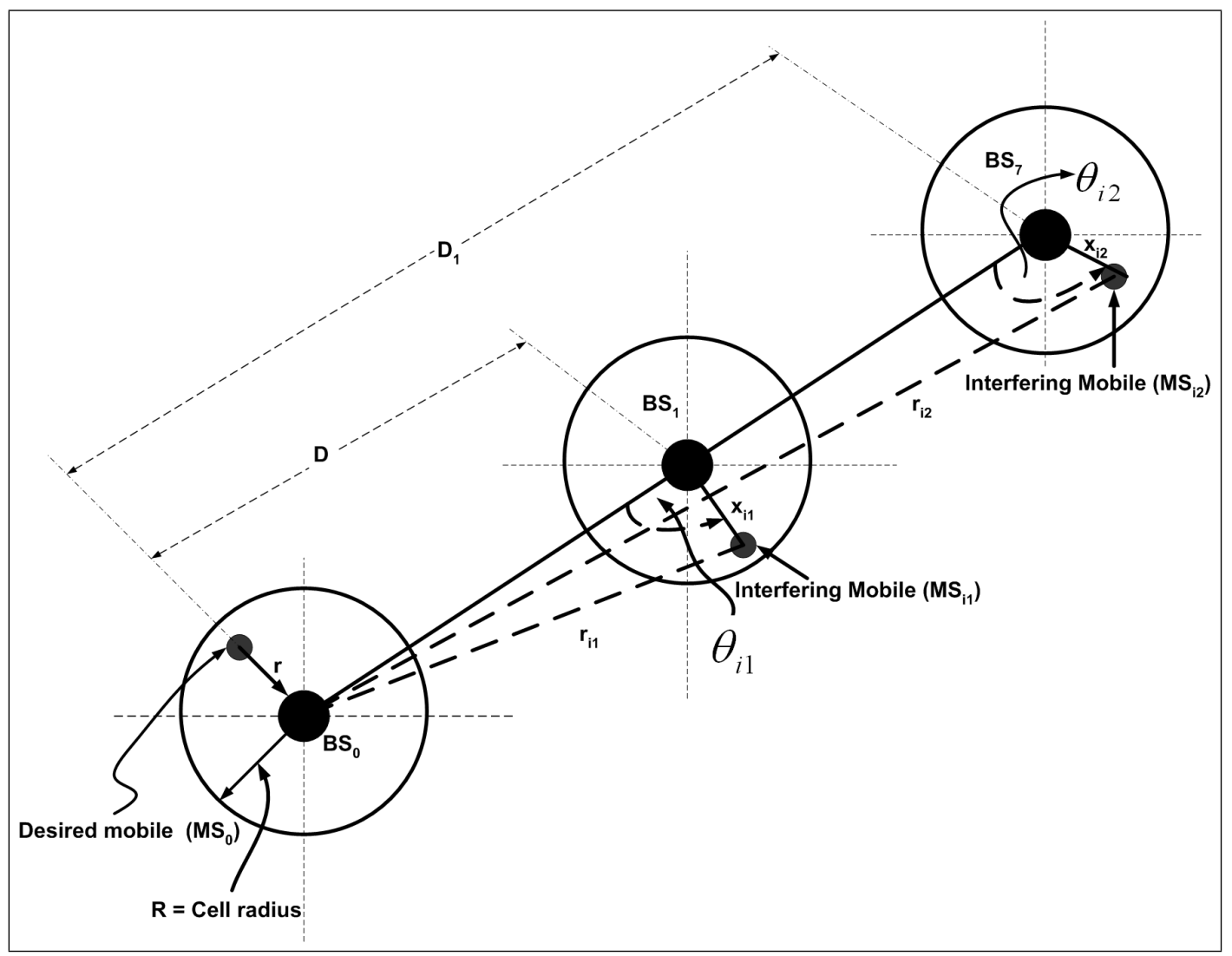

Figure 4.1: Geometry of desired user and interfering users in two co-channels cells; different tiers

1. $\mathrm{BS}_{0}$ : the reference cell

2. $\mathrm{BS}_{1}$ : first tier co-channel interfering cell

3. $\mathrm{BS}_{7}$ : second tier co-channel interfering cell

4. $\mathrm{D}$ : distance of separation between $\mathrm{BS}_{0}$ and $\mathrm{BS}_{1}$

5. $\mathrm{D}_{1}$ : distance of separation between $\mathrm{BS}_{0}$ and $\mathrm{BS}_{7}$

6. $\mathrm{r}$ : distance of separation between $\mathrm{MS}_{0}$ and $\mathrm{BS}_{0}$

7. $\mathrm{r}_{i 1}$ : distance of separation between $\mathrm{BS}_{0}$ and $\mathrm{X}_{i 1}$

8. $\mathrm{r}_{i 2}$ : distance of separation between $\mathrm{BS}_{0}$ and $\mathrm{X}_{i 2}$

9. $\theta_{i 1}$ : direction of $\mathrm{MS}_{i 1}$ travel referenced to a vector from $\mathrm{BS}_{0}$ to $\mathrm{BS}_{1}$

10. $\theta_{i 2}$ : direction of $\mathrm{MS}_{i 2}$ travel referenced to a vector from $\mathrm{BS}_{0}$ to $\mathrm{BS}_{7}$ 


\subsection{System Model for Information Capacity Analysis}

An information capacity performance comparison for the two interference model (conventional and proposed), is presented in this section. The analysis is based on the uplink area spectral efficiency. It is an extension of the work in [77], to include second tier cochannel interfering cells. The area spectral efficiency is defined as the achievable sum rate of all users in a cell per unit bandwidth per unit area. The analysis applies to TDMA cellular wireless communication systems. TDMA is the most representative of multiple access cellular wireless communication systems. The cellular system is considered to be fully loaded. That is, the cell's resources (serviced channels), are fully used. The number of interferers is constant and equals the sum of first and second tier co-channel interfering cell $N_{F I}+N_{S I}$. The cellular system used for the analysis is non-sectorized. It must be noted that our analysis can be extended to the downlink and sectorized cellular system.

\subsubsection{Area Spectral Efficiency - Conventional}

The area spectral efficiency for a fully loaded non-sectorized cellular wireless communication system is given by [77] as:

$$
A_{e}=\frac{\sum_{k=1}^{N_{s}} C_{k}}{\pi W(D / 2)^{2}}
$$

where $W$ is the total bandwidth allocated to each cell. The reuse distance $D[\mathrm{~m}]$ is defined as the distance between BS's using the same frequency set. $C_{k}$ is the achievable sum rate. $N_{s}$, is the number of service channel per cell.

The achievable sum rate $C_{k}$, is the Shannon capacity of the kth user. $C_{k}$, depends on $\gamma_{d}$, the received carrier to interference power ratio CIR of that user, and $W_{k}$ the bandwidth allocated to the user. The Shannon capacity formula assumes the interference has Gaussian characteristics $[104,105]$. Because it is assumed that the interference and signal power of the kth user vary with mobiles location and propagation conditions, $\gamma_{d}$ varies with time. Therefore, the average channel capacity of the kth user is given by

$$
\left\langle C_{k}\right\rangle=W_{k} \int_{0}^{+\infty} \log _{2}(1+\gamma) p_{\gamma}(\gamma) d \gamma
$$


where $\mathrm{p}_{\gamma}(\gamma)$, is the probability distribution function (PDF) of the average mean $\operatorname{CIR}\left(\gamma_{d}\right)$ of the kth user.

The transmission rate is assumed to be continuously adapted relative to the $C I R$ in such a manner that the $B E R$ goes to zero asymptotically. In equation (4.3) if all users are assigned the same bandwidth, $\left\langle C_{k}\right\rangle=(\langle C\rangle)$ becomes the same for all users. Therefore, the area spectral efficiency $\left\langle A_{e}\right\rangle$ is represented by

$$
\left\langle A_{e}\right\rangle=\frac{4 N_{s}\langle C\rangle}{\pi W D^{2}}=\frac{4 N_{s}\langle C\rangle}{\pi W R_{u}^{2} R^{2}},
$$

where $R_{u}$ is defined as the normalized reuse distance and is given by the ratio of reuse distance and cell radius $(D / R)$. In TDMA cellular wireless communication systems, the total bandwidth is allocated to only one active user per time slot $\left(N=1\right.$ and $\left.W_{k}=W\right)$. Substituting $W / N$ in equation (4.3) followed by substitution into equation (4.4) gives the $A S E$ as

$$
\left\langle A_{e}\right\rangle=\frac{4}{\pi R_{u}^{2} R^{2}} \int_{0}^{+\infty} \log _{2}(1+\gamma) p_{\gamma}(\gamma) d \gamma
$$

\subsubsection{Area Spectral Efficiency for Two-Tier Interference Model}

From section 4.2.1; users were assumed to be randomly located in their respective BS. Therefore, $\gamma_{d}$ is a random variable, and it depends on the random position of the user and the sums of interference from the first and second tier. Without power control the average-case interference configuration corresponds to the case where all the $N_{F I}$ and $N_{S I}$ co-channel interferes are at the center of their respectively BSs. At distances $r_{i 1}=D$ [m] and $r_{i 2}=2 D[\mathrm{~m}]$ from the desired mobile's BS. Note that we did not consider power control, because it is essential for direct sequence CDMA systems [106]. As shown in Figure 3.14, assuming that the transmitted power of all users are the same, and assuming distance dependent path loss, using equation (3.7) and equation (3.13), we obtain the 
desired MS's CIR as follows

$$
\begin{gathered}
\gamma_{d}\left(r, N_{F I}, N_{S I}\right)=\frac{\overline{P_{d}}(r)}{\sum_{i 1=1}^{N_{F I}} \overline{P_{i 1}}\left(r_{i 1}\right)+\sum_{i 2=1}^{N_{S I}} \overline{P_{i 2}}\left(r_{i 2}\right)}= \\
\frac{r^{-\alpha}(1+r / g)^{-\rho}}{\sum_{i 1=1}^{N_{F I}} r_{i 1}^{-\alpha}\left(1+r_{i 1} / g\right)^{-\rho}+\sum_{i 2=1}^{N_{S I}} r_{i 2}^{-\alpha}\left(1+r_{i 2} / g\right)^{-\rho}}= \\
\frac{r^{-\alpha}(1+r / g)^{-\rho}}{\sum_{i 1=1}^{N_{F I}} D^{-\alpha}(1+D / g)^{-\rho}+\sum_{i 2=1}^{N_{S I}}(2 D)^{-\alpha}(1+(2 D) / g)^{-\rho}}= \\
\frac{r^{-\alpha}(1+r / g)^{-\rho}}{\left(\frac{2^{\alpha} \cdot N_{F I}+N_{S I}}{N_{F I} \cdot N_{S I}}\right) \cdot\left(\frac{R_{u} R}{r}\right)^{\alpha}\left(\frac{g+2 R_{u} R}{g+r}\right)^{\rho}},
\end{gathered}
$$

where $r$ is the distance from desired MS's to desired BS's. $D$ is the distance between two BSs' using the same frequency, and it is the product of the cell size radius and normalized reuse distance $\left(R_{u} \times R\right)$. Since $\gamma_{d}$ is a function of $r$, the desired user capacity $\left\langle C\left(r, N_{F I}, N_{S I}\right)\right\rangle$ is given by

$$
\left\langle C\left(r, N_{F I}, N_{S I}\right)\right\rangle=W_{o} \log _{2}\left(1+\gamma\left(r, N_{F I}, N_{S I}\right)\right),
$$

Substituting equation (4.7) in equation (4.4) yields the $A S E$ conditioned on the desired mobile position $r$, for a fully-loaded system. Integrating equation (4.7) over the desired user's position PDF, equation (4.1) yields the average $A S E$ for the average interference configuration as:

$$
\left\langle A_{e}\left(r, N_{F I}, N_{S I}\right)\right\rangle=\frac{4}{\pi R_{u}^{2} R^{2}} \int_{R_{0}}^{R} \log _{2}(1+\gamma) p_{\gamma}(r) d r,
$$

The equation (4.8), shows that the average area spectral efficiency mainly depends on the mean $C I R$, which is a function of random locations of the MS. This makes the ASE mathematically intractable to solve. A computer simulation is therefore used to solve it. 


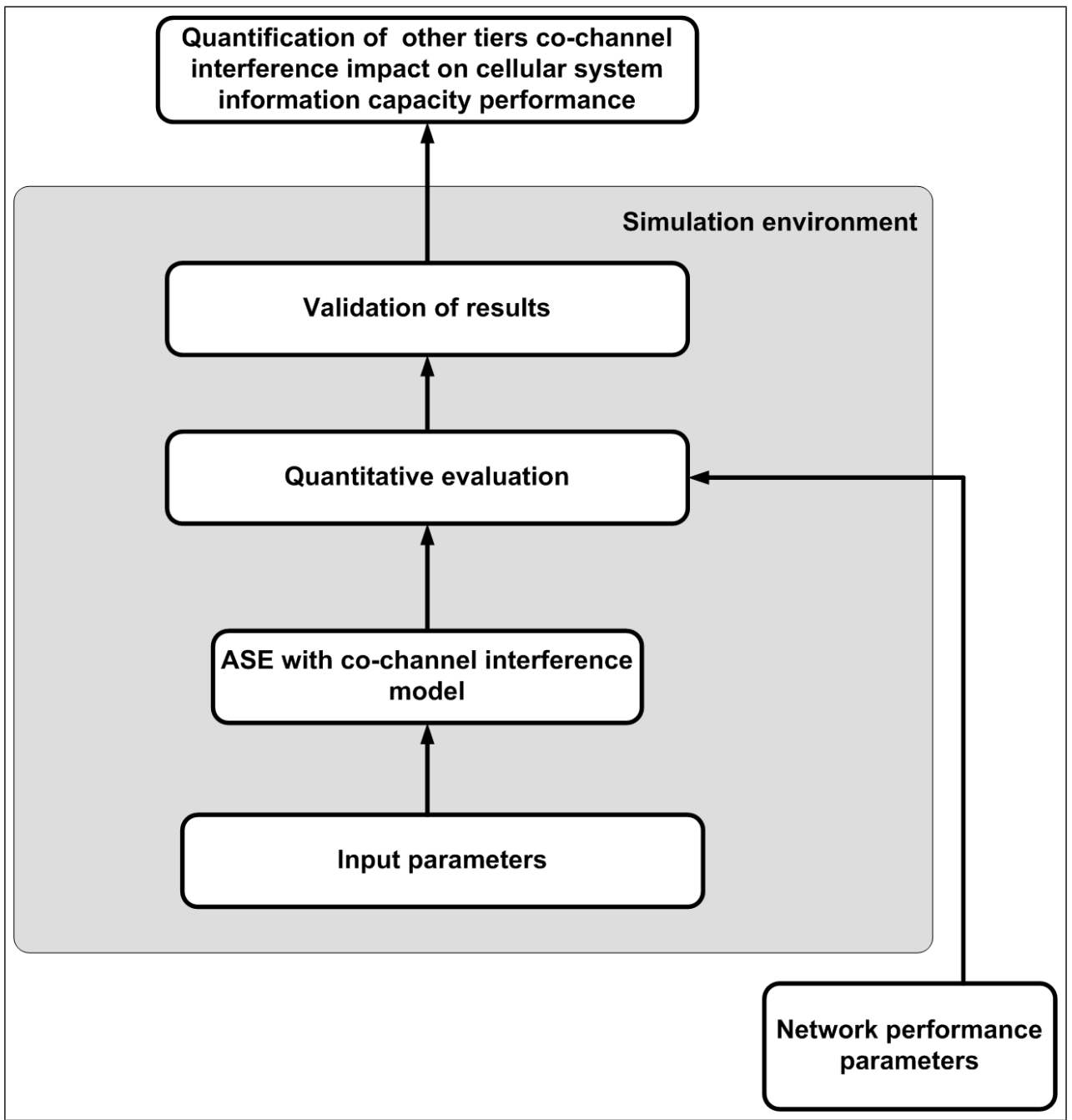

Figure 4.2: Integrated simulation environment for information capacity analysis

\subsection{Simulation Model-Information Capacity Comparison}

Computer simulations, were carried out to compare the cellular system information capacity performance for the conventional and our proposed two tier interference model. The comparison was performed, for different carrier frequencies and cell size radii. Computer simulations were used, because the analysis is mathematically intractable to solve explicitly. In order that this simulation model can be used in a variety of tasks, flexibility is provided in terms of its inputs and outputs. Figure 4.2, shows the simulation environment. The objective of this simulation is to generate sufficient data to examine the area spectral efficiency, and related system and propagation loss parameters as a function of cell size radius and normalized reuse distance. 


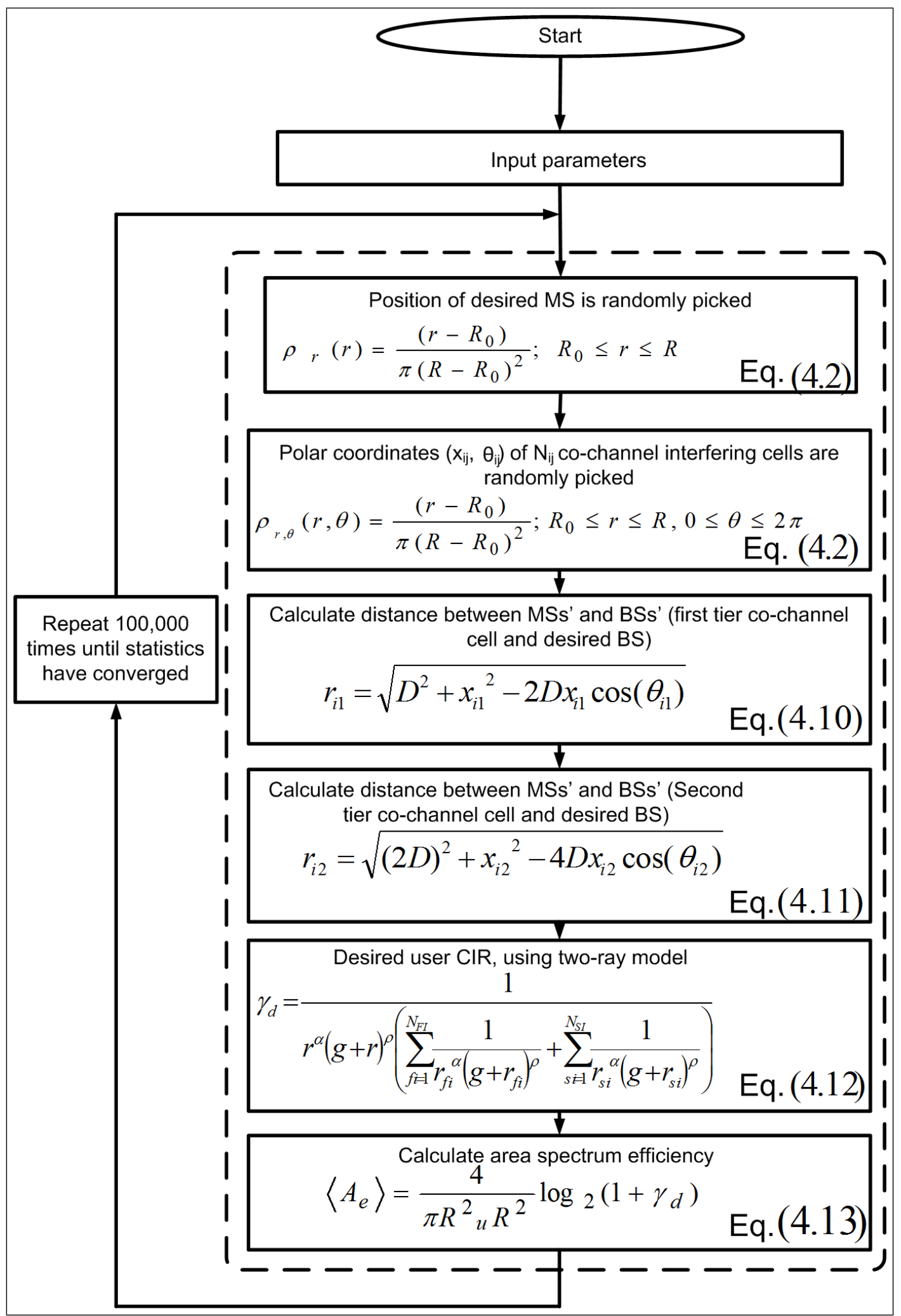

Figure 4.3: Flow chart for information capacity comparison simulations 


\subsubsection{Simulation Setup for One and Two Tier Interference Model}

A uniform distribution is assumed for the initial spatial location of the desired user as described in subsection 4.2.1. This assumption is valid, because in a cellular wireless network, the relative position of mobile users in cells varies randomly, giving on the average an approximately uniform distribution of possible positions. The polar coordinates $\left(x_{i 1}, \theta_{i 1}\right)$ and $\left(x_{i 2}, \theta_{i 2}\right)$ of the $N_{f i}$ and $N_{s i}$ co-channel interfering mobiles in the first and second tier are also assumed to be uniformly distributed according to equation 4.1. The limits of the distribution are in the range of $[0,2 \pi]$, with respect to current position of MS. The geometry for calculation of interference for the simulation is shown in Figure 4.1. How the simulation proceeds is illustrated by a flow chart in Figure 4.3. The distance $r_{f i}$ for each co-channel interferer from the first tier to the desired BS for the simulation is calculated by

$$
r_{f i}=\sqrt{D^{2}+x_{i 1}^{2}-2 D x_{i 1} \cos \left(\theta_{i 1}\right)}
$$

The distance $r_{s i}$ for each co-channel interferer from the second tier to the desired BS for the simulation is calculated by

$$
r_{s i}=\sqrt{(2 D)^{2}+x_{i 2}^{2}-4 D x_{i 2} \cos \left(\theta_{i 2}\right)}
$$

The two-slope path loss model in equation (3.6), is used to calculate the average received signal power for the desired MS $\left(P_{d}\right)$, and interfering MSs' ( $P_{f i}$ 's and $P_{s i}$ 's), in the first and second tier of co-channel cells for the simulation. Note that the average received signal power based on the two-slope path loss model is distance dependent. Also $\left(\gamma_{d}\right)$ is a function of distance $r$. Therefore, the two-slope path loss model is still suitable for the modelling the second tier co-channel interference. For the simulation, $\operatorname{CIR}\left(\gamma_{d}\right)$, is calculated by

$$
\gamma_{d}=\frac{1}{r^{\alpha}(g+r)^{\rho}\left(\sum_{f i=1}^{N_{F I}} \frac{1}{r_{f i}^{\alpha}\left(g+r_{f i}\right)^{\rho}}+\sum_{s i=1}^{N_{S I}} \frac{1}{r_{s i}^{\alpha}\left(g+r_{s i}\right)^{\rho}}\right)}
$$

where $r$, is the distance between a desired MS and its BS. $N_{F I}$ and $N_{S I}$, are the number of co-channel interfering cells in the first and second tier. $\alpha$ and $\rho$, are the basic and extra path loss exponents. The area spectral efficiency $\left\langle A_{e}\right\rangle$, for a desired MS is then calculated 
Simulation Model-Information Capacity Comparison

\begin{tabular}{|l|l|}
\hline Parameters & Values \\
\hline Cell radius & 100 to $1000 \mathrm{~m}$ \\
\hline Path loss exponent $(\alpha)$ & $2,2.5,2.8$ and 3 \\
\hline Additional path loss exponent $(\rho)$ & 4 \\
\hline Reference distance $\left(\mathrm{d}_{0}\right)$ & $1 \mathrm{~m}$ \\
\hline Path loss constant $(\mathrm{K})$ & $20 \log _{10}\left(\lambda_{c} / 4 \pi d_{0}\right) \mathrm{dB}$ \\
\hline MS transmission power $\left(\mathrm{P}_{t}\right)$ & $1 \mathrm{~W}$ \\
\hline BS antenna height $\left(\mathrm{h}_{b}\right)$ & 15,35 and $55 \mathrm{~m}[87,107,108]$ \\
\hline MS antenna height $\left(\mathrm{h}_{m}\right)$ & 1.5 and $1.8 \mathrm{~m}[87,107,108]$ \\
\hline Mobile Distribution & Uniform and Random \\
\hline Interference & First and Second Tiers \\
\hline Carrier frequencies $f_{c}$ & $0.9,2,3.35,8.45$ and $15.75 \mathrm{GHz}[77,85]$ \\
\hline Frequency reuse factor & $4[77]$ \\
\hline
\end{tabular}

Table 4.1: Simulation Parameters for Information Capacity Comparison

by

$$
\left\langle A_{e}\right\rangle=\frac{4}{\pi R_{u}^{2} R^{2}} \log _{2}\left(1+\gamma_{d}\right) .
$$

where $R_{u}$ is the normalized reuse distance and $R$ is the cell size radius. The system parameters used in the simulations are: basic path loss exponent $\alpha=2$, extra path loss exponent $\rho=2$, normalized reuse distance $R_{u}=4$, cell radii $R=100,200$ and $500 \mathrm{~m}$, Bs antenna $h_{b}=15 \mathrm{~m}$ and MS antenna height $h_{m}=1.5 \mathrm{~m}$, and carrier frequencies $f_{c}=0.9,2,3.35$, 8.45, and $15.75 \mathrm{GHz}$. It has been reported that microwave bands, $(3-60 \mathrm{GHz})$ are to be used in future wireless communication systems $[109,110]$. Now, it must be stated that the physics of radio wave propagation tends to change at carrier frequencies above $20 \mathrm{GHz}$, when rain attenuation begins to be significant $[111,112]$, therefore (i) for the work to be practically applicable ahead of future wireless application, we consider carrier frequencies below $20 \mathrm{GHz}$, (ii) in order not to divert into other research challenges relating to future wireless system, such as propagation modelling [109], carrier frequencies $f_{c}=3.35,8.45$ and $15.75 \mathrm{GHz}$ were considered for the analysis and simulation in this work. These carrier frequencies were considered, because the propagation modelling relating to those carrier frequencies, $f_{c}=3.35,8.45$ and $15.75 \mathrm{GHz}$ have already be done [85]. We present a sum- 
mary of the simulation parameters in Table 4.1. The main emphasis of the simulation is to reflect, as far as possible, a truly typical case of a cellular wireless communication system. Therefore, the simulation process needs to be repeated a large number of times. Repeating the simulation, will minimize the influence of initial conditions and the variation of the stochastic processes. The simulation process was repeated 100000 times for it to reach a steady state condition. The value $\left\langle A_{e}\right\rangle$, was then estimated by taking the average of all the observations of $A_{e}$.

\subsection{Numerical Results-Information Capacity Comparison}

In this section, we present simulation results for the information capacity performance comparison between the conventional and proposed interference model, for cellular wireless communication systems. Our simulation results collaborates results in [77]. The results show that decreasing reuse distance and reducing cell size radius leads to an increase in the information capacity of the cellular wireless communication system. Figures 4.4 4.8, show plots of average area spectral efficiency, $A S E$ versus normalized reuse distance $R_{u}$, for different carrier frequencies $f_{c}$ and cell size radius $R$. The plots show that at $R=$ $500 \mathrm{~m}$, for different $f_{c}=0.9,2,3.35,8.45$, and $15.75 \mathrm{GHz}$, the information capacity for the two interference models overlay each other. This means that for cell size radius $R \geq$ $500 \mathrm{~m}$ the second tier co-channel interfering cells did not affect the information capacity performance of the cellular wireless communication system.

Figure 4.4, shows that when $f_{c}=900 \mathrm{MHz}$ and $R=200 \mathrm{~m}$ for a normalized reuse distance $R_{u}=4$, there was a $4 \%$ decrease in information capacity between the two interference models and for $100 \mathrm{~m}$ the decrease was $6 \%$. Figure 4.5, provides the decrease in information capacity for $f_{c}=2 \mathrm{GHz}$. The curve shows a decrease of $8 \%$ in $A S E$ for $R=100 \mathrm{~m}$ and $R_{u}=4$. When $R=200 \mathrm{~m}$ the decrease was $6 \%$. The curve for $f_{c}=3.35$ GHz is provided in Figure 4.6. The decrease in ASE for $R=100$, and $200 \mathrm{~m}$ for $R_{u}=4$ is 10.53 and $7.5 \%$. Figure 4.7, shows that when $f_{c}=8.45 \mathrm{GHz}$ and $R_{u}=4$, for $R=100 \mathrm{~m}$ the decrease in $A S E$ between the conventional and proposed interference model was 13.8 $\%$. For $R=200 \mathrm{~m}$ the decrease was $10.82 \%$. The curve for carrier frequency $f_{c}=15.75$ GHz is provided in Figure 4.7. The curves show that at a normalized reuse distance 


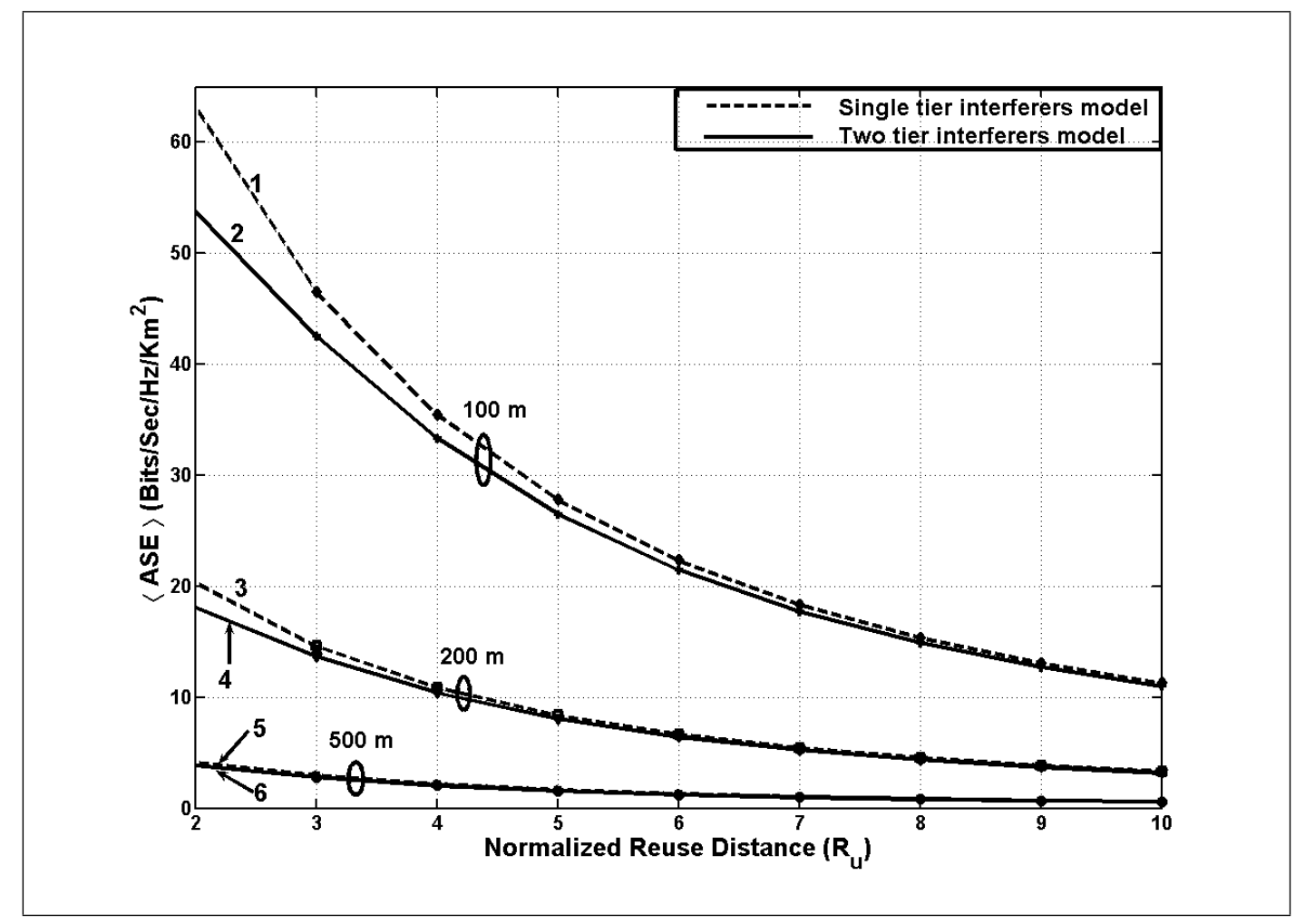

Figure 4.4: Comparison of the average area spectral efficiency, $A S E$ for single and two tier co-channel interference model for different normalized reuse distances $R_{u}$ and cell size radius $R$. (Fully loaded non-sectorized cellular system; carrier frequency $f_{c}=900 \mathrm{MHz}$; path loss exponent $\alpha=2$ and extra path loss exponent $\rho=2$; BS antenna height $h_{b}=15$ $\mathrm{m}$ and MS antenna height $h_{m}=1.5 \mathrm{~m}$; number of co-channel interfering cells in the first tier, $N_{F I}=6$, and second tier $N_{S I}=12$ )

1. $A S E$ for conventional single tier co-channel interference model, $\mathrm{R}=100 \mathrm{~m} \mathrm{[77]}$

2. $A S E$ for proposed two tier co-channel interference model, $\mathrm{R}=100 \mathrm{~m}$

3. $A S E$ for conventional single tier co-channel interference model, $\mathrm{R}=200 \mathrm{~m}$ [77]

4. $A S E$ for proposed two tier co-channel interference model, $\mathrm{R}=200 \mathrm{~m}$

5. $A S E$ for conventional single tier co-channel interference model, $\mathrm{R}=500 \mathrm{~m}$ [77]

6. $A S E$ for proposed two tier co-channel interference model, $\mathrm{R}=500 \mathrm{~m}$ 


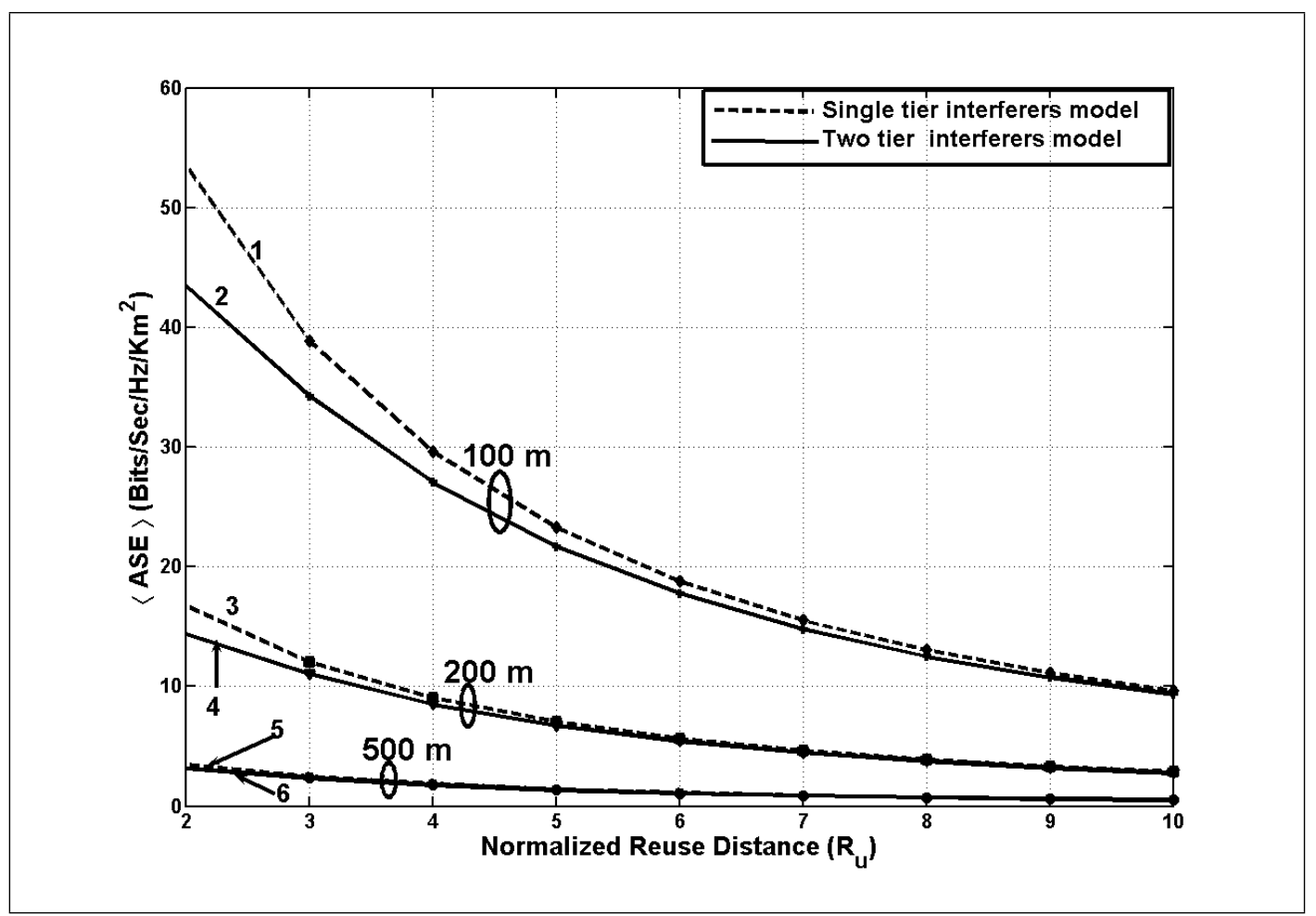

Figure 4.5: Comparison of the average area spectral efficiency, ASE for single and two tier co-channel interference model for different normalized reuse distances $R_{u}$ and cell size radius $R$. (Fully loaded non-sectorized cellular system; carrier frequency $f_{c}=2 \mathrm{GHz}$; path loss exponent $\alpha=2$ and extra path loss exponent $\rho=2$; BS antenna height $h_{b}=15$ $\mathrm{m}$ and MS antenna height $h_{m}=1.5 \mathrm{~m}$; number of co-channel interfering cells in the first tier, $N_{F I}=6$, and second tier $N_{S I}=12$ )

1. $A S E$ for conventional single tier co-channel interference model, $\mathrm{R}=100 \mathrm{~m} \mathrm{[77]}$

2. $A S E$ for proposed two tier co-channel interference model, $\mathrm{R}=100 \mathrm{~m}$

3. $A S E$ for conventional single tier co-channel interference model, $\mathrm{R}=200 \mathrm{~m}$ [77]

4. $A S E$ for proposed two tier co-channel interference model, $\mathrm{R}=200 \mathrm{~m}$

5. $A S E$ for conventional single tier co-channel interference model, $\mathrm{R}=500 \mathrm{~m}$ [77]

6. $A S E$ for proposed two tier co-channel interference model, $\mathrm{R}=500 \mathrm{~m}$ 


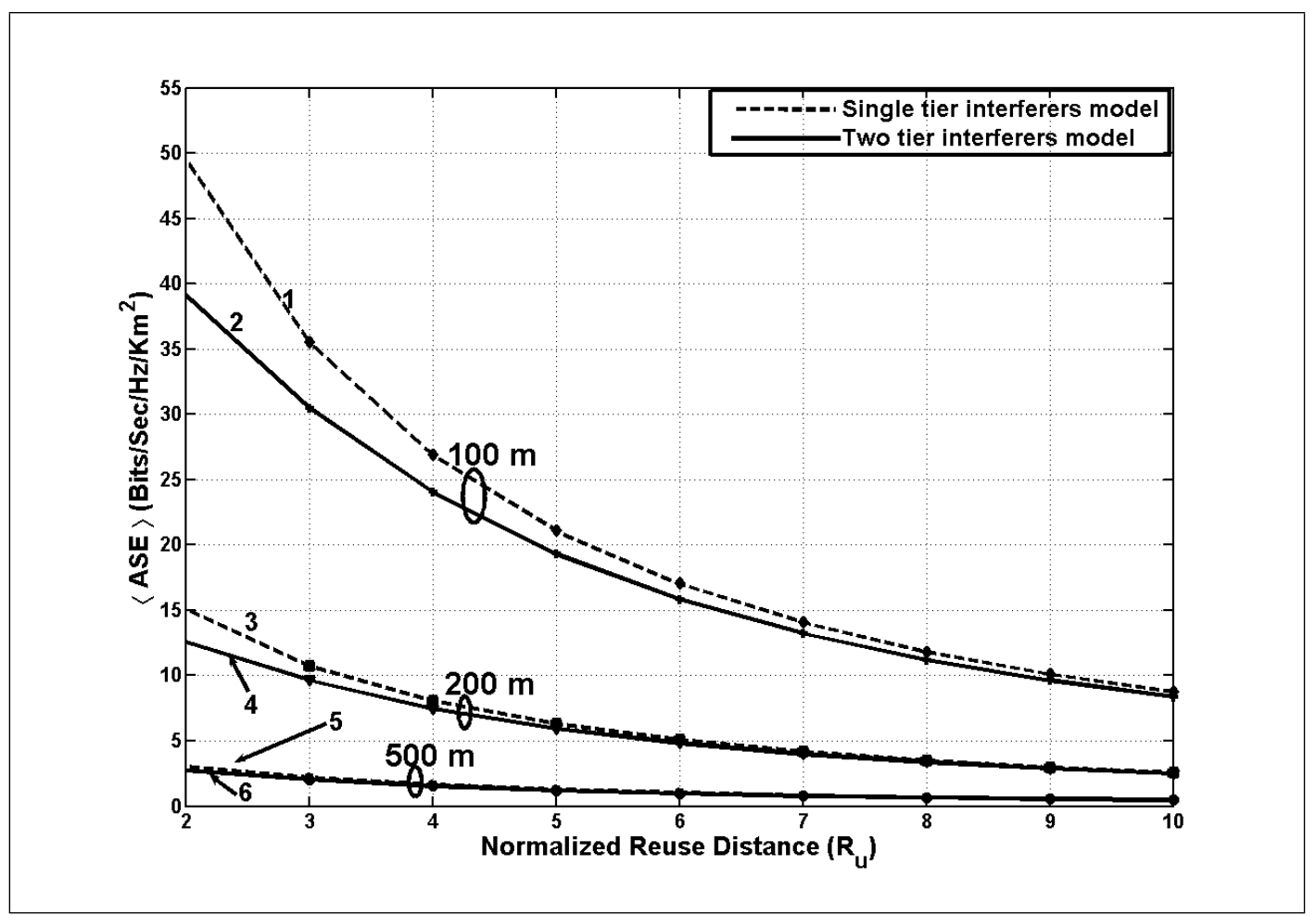

Figure 4.6: Comparison of the average area spectral efficiency, ASE for single and two tier co-channel interference model for different normalized reuse distances $R_{u}$ and cell size radius $R$. (Fully loaded non-sectorized cellular system; carrier frequency $f_{c}=3.35$ GHz; path loss exponent $\alpha=2$ and extra path loss exponent $\rho=2$; BS antenna height $h_{b}$ $=15 \mathrm{~m}$ and MS antenna height $h_{m}=1.5 \mathrm{~m}$; number of co-channel interfering cells in the first tier, $N_{F I}=6$, and second tier $N_{S I}=12$ )

1. $A S E$ for conventional single tier co-channel interference model, $\mathrm{R}=100 \mathrm{~m}$ [77]

2. $A S E$ for proposed two tier co-channel interference model, $\mathrm{R}=100 \mathrm{~m}$

3. $A S E$ for conventional single tier co-channel interference model, $\mathrm{R}=200 \mathrm{~m}$ [77]

4. $A S E$ for proposed two tier co-channel interference model, $\mathrm{R}=200 \mathrm{~m}$

5. $A S E$ for conventional single tier co-channel interference model, $\mathrm{R}=500 \mathrm{~m}$ [77]

6. $A S E$ for proposed two tier co-channel interference model, $\mathrm{R}=500 \mathrm{~m}$ 


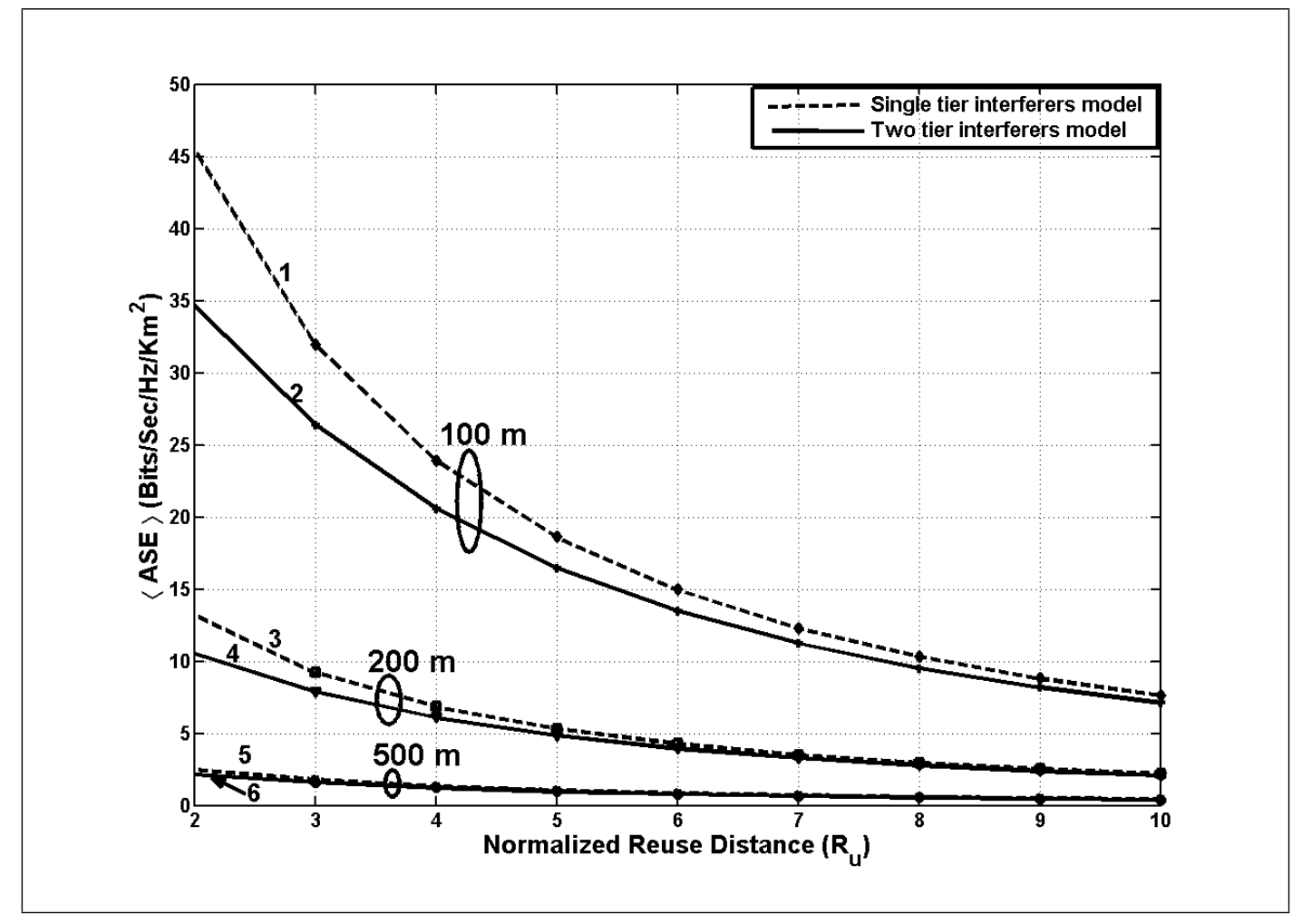

Figure 4.7: Comparison of the average area spectral efficiency, $A S E$ for single and two tier co-channel interference model for different normalized reuse distances $R_{u}$ and cell size radius $R$. (Fully loaded non-sectorized cellular system; carrier frequency $f_{c}=8.45$ GHz; path loss exponent $\alpha=2$ and extra path loss exponent $\rho=2$; BS antenna height $h_{b}$ $=15 \mathrm{~m}$ and MS antenna height $h_{m}=1.5 \mathrm{~m}$; number of co-channel interfering cells in the first tier, $N_{F I}=6$, and second tier $N_{S I}=12$ )

1. $A S E$ for conventional single tier co-channel interference model, $\mathrm{R}=100 \mathrm{~m} \mathrm{[77]}$

2. $A S E$ for proposed two tier co-channel interference model, $\mathrm{R}=100 \mathrm{~m}$

3. $A S E$ for conventional single tier co-channel interference model, $\mathrm{R}=200 \mathrm{~m}$ [77]

4. $A S E$ for proposed two tier co-channel interference model, $\mathrm{R}=200 \mathrm{~m}$

5. $A S E$ for conventional single tier co-channel interference model, $\mathrm{R}=500 \mathrm{~m}$ [77]

6. $A S E$ for proposed two tier co-channel interference model, $\mathrm{R}=500 \mathrm{~m}$ 


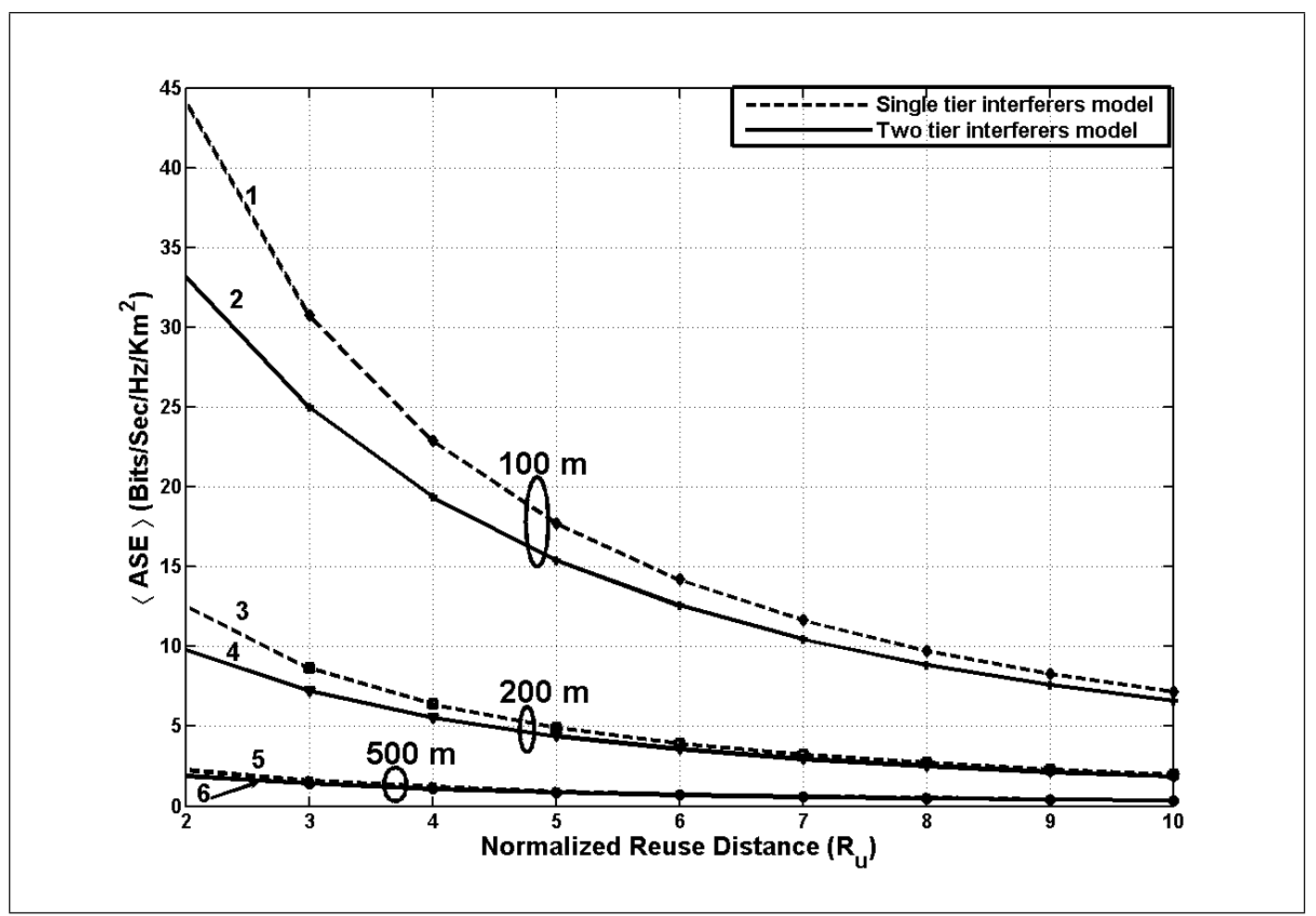

Figure 4.8: Comparison of the average area spectral efficiency for single and two tier cochannel interference model for different normalized reuse distances $R_{u}$ and cell size radius $R$. (Fully loaded non-sectorized cellular system; carrier frequency $f_{c}=15.75 \mathrm{GHz}$; path loss exponent $\alpha=2$ and extra path loss exponent $\rho=2$; BS antenna height $h_{b}=15 \mathrm{~m}$ and MS antenna height $h_{m}=1.5 \mathrm{~m}$; number of co-channel interfering cells in the first tier, $N_{F I}$ $=6$, and second tier $N_{S I}=12$ )

1. $A S E$ for conventional single tier co-channel interference model, $\mathrm{R}=100 \mathrm{~m} \mathrm{[77]}$

2. $A S E$ for proposed two tier co-channel interference model, $\mathrm{R}=100 \mathrm{~m}$

3. $A S E$ for conventional single tier co-channel interference model, $\mathrm{R}=200 \mathrm{~m}$ [77]

4. $A S E$ for proposed two tier co-channel interference model, $\mathrm{R}=200 \mathrm{~m}$

5. $A S E$ for conventional single tier co-channel interference model, $\mathrm{R}=500 \mathrm{~m}$ [77]

6. $A S E$ for proposed two tier co-channel interference model, $\mathrm{R}=500 \mathrm{~m}$ 
Numerical Results-Information Capacity Comparison

\begin{tabular}{|l|l|l|}
\hline \multicolumn{3}{|c|}{$\mathrm{h}_{m}=1.5 \mathrm{~m}, \mathrm{~h}_{b}=15 \mathrm{~m}, \alpha=2, \rho=2, \mathrm{R}_{u}=4$} \\
\hline Cell Size Radius, $R$ & Carrier Frequency, $f_{c}$ & Percentage Decrease (\%) \\
\hline $100 \mathrm{~m}$ & $900 \mathrm{MHz}$ & 6.0 \\
& $2 \mathrm{GHz}$ & 8.55 \\
& $3.35 \mathrm{GHz}$ & 10.53 \\
& $8.45 \mathrm{GHz}$ & 13.77 \\
& $15.75 \mathrm{GHz}$ & 15.32 \\
\hline \multirow{5}{*}{$200 \mathrm{~m}$} & $900 \mathrm{MHz}$ & 4.24 \\
& $2 \mathrm{GHz}$ & 5.98 \\
& $3.35 \mathrm{GHz}$ & 7.52 \\
& $8.45 \mathrm{GHz}$ & 10.82 \\
& $15.75 \mathrm{GHz}$ & 12.87 \\
\hline $500 \mathrm{~m}$ & $900 \mathrm{MHz}$ & - \\
& $2 \mathrm{GHz}$ & - \\
& $3.35 \mathrm{GHz}$ & - \\
\hline & $8.45 \mathrm{GHz}$ & - \\
\hline
\end{tabular}

Table 4.2: Percentage Decrease in Area Spectral Efficiency between single and two tier Interference Model for Different Cell Size Radius $R$

$R_{u}=4$, for cell size radius $R=100$, and $200 \mathrm{~m}$ the decrease in information capacity is 15.32 and $12.87 \%$. These results show that as carrier frequency increases and cell size radius decreases the percentage decrease in $A S E$ between the conventional and proposed cochannel interference model increases. A summary of the results is presented in Table 4.2. From the results, it can be concluded that at higher carrier frequencies and smaller cell size radii the second tier co-channel interfering cell become active. The interference from the second tier co-channel interfering cells, therefore causes a reduction in the information capacity of the cellular wireless communication system.

\subsubsection{Effect of Basic Path Loss Exponent-Two Tier Interference}

In this section, we present numerical results on the impact of basic path loss exponent on the information capacity performance of a cellular wireless system. The aim of the 
results presented in this section is to make clear the influence of basic path loss exponent on the information capacity performance of emerging and future cellular wireless systems. Our simulation compares results for the conventional model presented in [77] (first tier co-channel interfering cell only), to our proposed model (first and second co-channel interfering cell), presented in this work (4.8).

Figure 4.9 - 4.12, shows the curves, for area spectral efficiency $A S E$ versus cell size radius $R$. The curves, are for different basic path loss exponent $\alpha$ and carrier frequencies $f_{c}$. The figure quantify the fact that decreasing cell size increases the $A S E$. The curves show that the $A S E$ increases as the basic path loss exponent $\alpha$ becomes larger, which is in agreement with existing work [77].

The curves in Figure 4.9, show that for basic path loss exponent $\alpha=2$, at $f_{c}=$ 0.9 and $2 \mathrm{GHz}$, the second tier co-channel interfering cells have no effect on the ASE. Therefore, for both the conventional and proposed interference model the curves overlay each other. For $f_{c}=3.35 \mathrm{GHz}$, and $R=100$ and $200 \mathrm{~m}$ there was a decrease of $11.13 \%$ and $4.81 \%$ in $A S E$ between the conventional and proposed model. For $300 \mathrm{~m} \leq R \leq 1000 \mathrm{~m}$ the decrease in $A S E \leq 2.98 \%$. When $f_{c}=8.45 \mathrm{GHz}$ for $500 \mathrm{~m} \leq R \leq 1000 \mathrm{~m}$ the decrease in $A S E$ was between $1.92-3.80 \%$. For $100 \mathrm{~m} \leq R \leq 400 \mathrm{~m}$ the decrease in $A S E$ between the two models is between $4.8-17.23 \%$.

Figure $4.10-4.12$, shows the curves for basic path loss exponent $\alpha=2.5,2.8$ and 3 at different carrier frequencies. The curves show that for $f_{c}=0.9,2$ and $3.35 \mathrm{GHz}$ there were no decrease in information capacity of the cellular wireless network. For $\alpha=2.5$ at $f_{c}=8.45 \mathrm{GHz}$, when cell size radius $R$ is between $100-500 \mathrm{~m}$ the decrease in $A S E$ is between $2.37-11.804 \%$. For $R>500 \mathrm{~m}$ there was no decrease in $A S E$ between the two models. At $f_{c}=15.75 \mathrm{GHz}$, the decrease was $14.23 \%$ at $100 \mathrm{~m}, 8.52 \%$ at $200 \mathrm{~m}$ and $3.9 \%$ at $500 \mathrm{~m}$ and less for $R>500 \mathrm{~m}$. The curves show that for $\alpha=2.8$ and 3 at 8.45 and 15.75 $\mathrm{GHz}$ the decrease was between $2.44-11.3 \%$ for $100 \leq R \leq 500$.

Figures $4.10,4.11$ and 4.12 , show that as $\alpha$ increases the decrease in information capacity between the two interference model reduces, because for larger basic path loss exponent $(\alpha)$, interference from MSs before and after the breakpoint distance become less, even at higher carrier frequencies. This is based on the fact that from the path loss model the received signal power is inversely proportional to (distance $)^{\alpha}$. We therefore conclude 


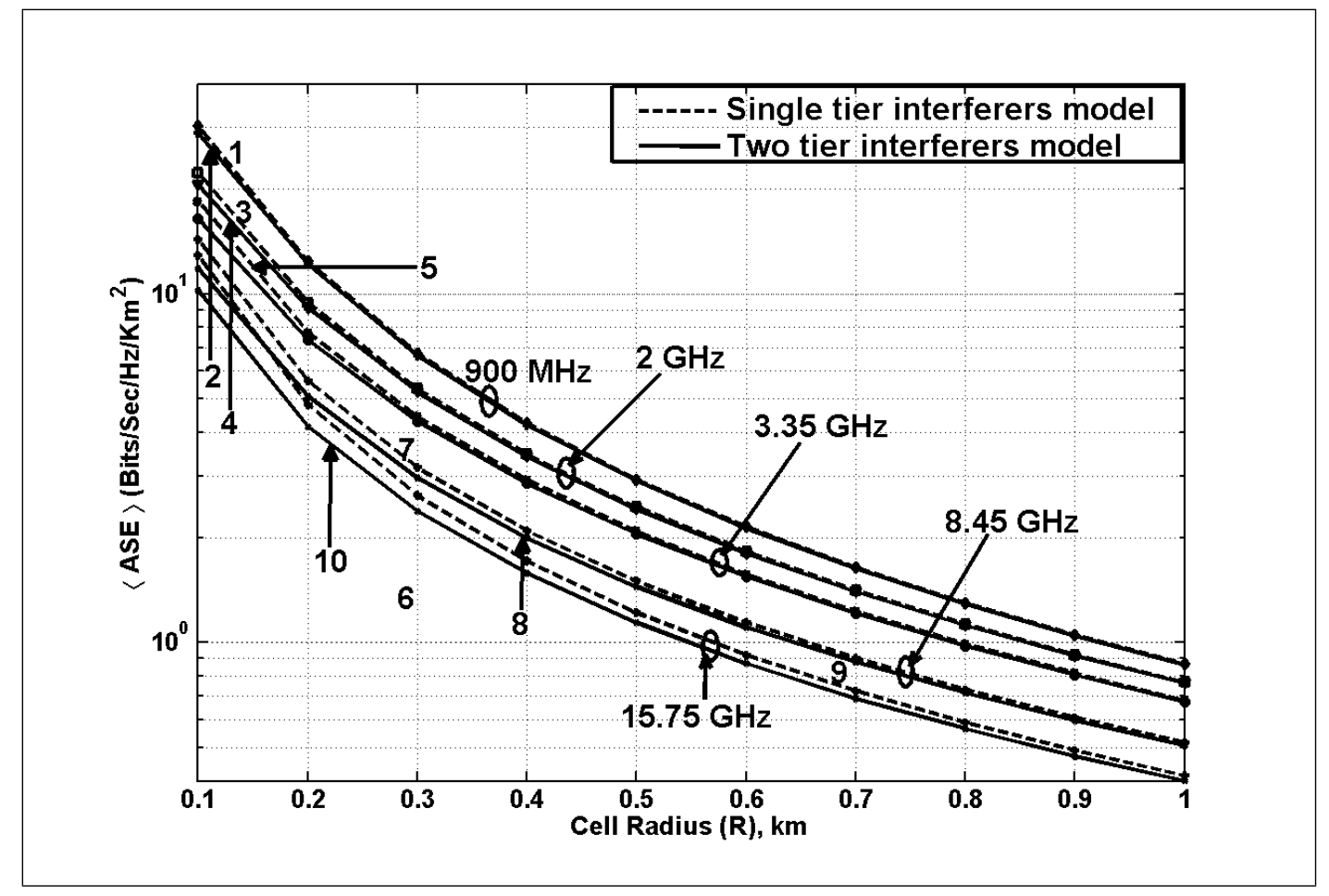

Figure 4.9: Area spectral efficiency versus cell size radius for path loss exponent, $\alpha=2$, and different carrier frequencies $f_{c}$. (Fully-loaded system with first and second tier cochannel interfering cells, $N_{F I}=6$ and $N_{S I}=12$; extra path loss exponent, $\rho=4$; MS and BS antenna heights : $h_{m}=1.5 \mathrm{~m}$ and $h_{b}=15 \mathrm{~m}$ )

1 Single tier co-channel interfering model $\left(f_{c}=900 \mathrm{MHz}\right)$ [77]

2 Two tier co-channel interfering model $\left(f_{c}=900 \mathrm{MHz}\right)$

3 Single tier co-channel interfering model $\left(f_{c}=2 \mathrm{GHz}\right)$ [77]

4 Two tier co-channel interfering model $\left(f_{c}=2 \mathrm{GHz}\right)$

5 Single tier co-channel interfering model $\left(f_{c}=3.35 \mathrm{GHz}\right)$ [77]

6 Two tier co-channel interfering model $\left(f_{c}=3.35 \mathrm{GHz}\right)$

7 Single tier co-channel interfering model $\left(f_{c}=8.45 \mathrm{GHz}\right)$ [77]

8 Two tier co-channel interfering model $\left(f_{c}=8.45 \mathrm{GHz}\right)$

9 Single tier co-channel interfering model $\left(f_{c}=15.75 \mathrm{GHz}\right)$ [77]

10 Two tier co-channel interfering model $\left(f_{c}=15.75 \mathrm{GHz}\right)$ 


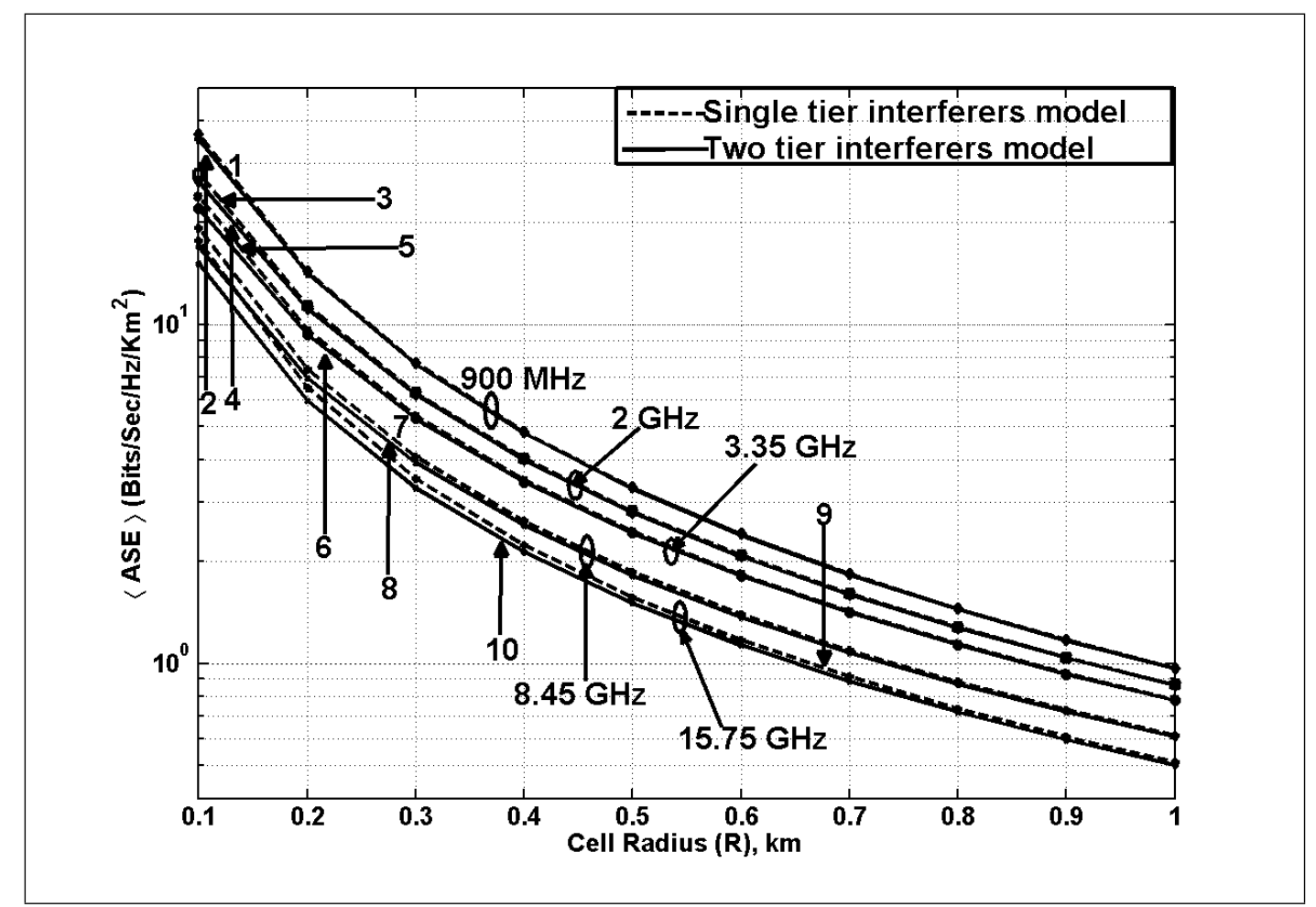

Figure 4.10: Average uplink area spectral efficiency versus cell size radius for path loss exponent, $\alpha=2.5$, and different carrier frequencies $f_{c}$. (Fully-loaded system with first and second tier co-channel interfering cells, $N_{F I}=6$ and $N_{S I}=12$; extra path loss exponent, $\rho=4$; MS and BS antenna heights : $h_{m}=1.5 \mathrm{~m}$ and $h_{b}=15 \mathrm{~m}$ )

1 Single tier co-channel interfering model $\left(f_{c}=900 \mathrm{MHz}\right)$ [77]

2 Two tier co-channel interfering model $\left(f_{c}=900 \mathrm{MHz}\right)$

3 Single tier co-channel interfering model $\left(f_{c}=2 \mathrm{GHz}\right)$ [77]

4 Two tier co-channel interfering model $\left(f_{c}=2 \mathrm{GHz}\right)$

5 Single tier co-channel interfering model $\left(f_{c}=3.35 \mathrm{GHz}\right)$ [77]

6 Two tier co-channel interfering model $\left(f_{c}=3.35 \mathrm{GHz}\right)$

7 Single tier co-channel interfering model $\left(f_{c}=8.45 \mathrm{GHz}\right)[77]$

8 Two tier co-channel interfering model $\left(f_{c}=8.45 \mathrm{GHz}\right)$

9 Single tier co-channel interfering model $\left(f_{c}=15.75 \mathrm{GHz}\right)$ [77]

10 Two tier co-channel interfering model $\left(f_{c}=15.75 \mathrm{GHz}\right)$ 


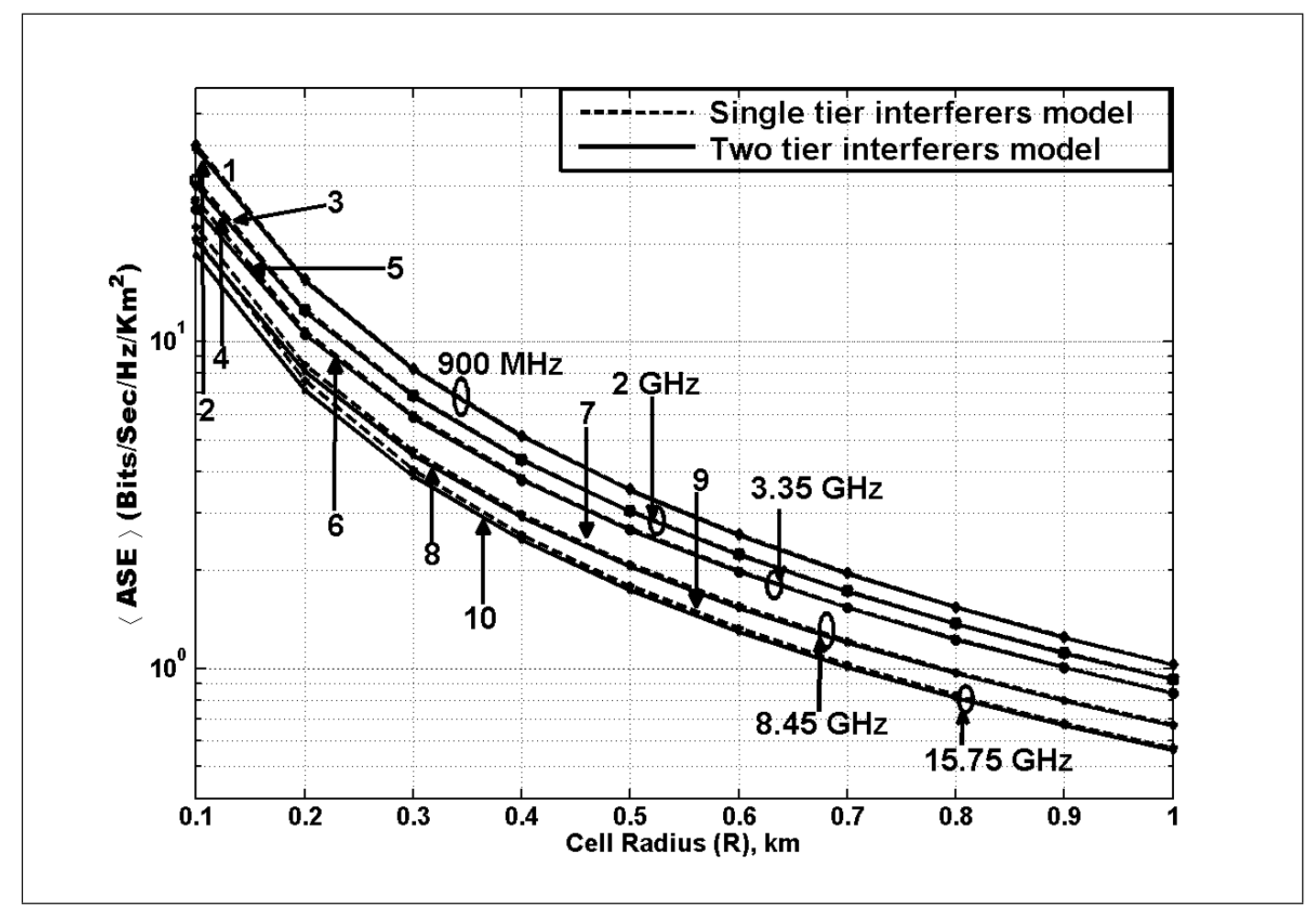

Figure 4.11: Average uplink area spectral efficiency versus cell size radius for path loss exponent, $\alpha=2.8$, and different carrier frequencies $f_{c}$. (Fully-loaded system with first and second tier co-channel interfering cells, $N_{F I}=6$ and $N_{S I}=12$; extra path loss exponent, $\rho=4$; MS and BS antenna heights $: h_{m}=1.5 \mathrm{~m}$ and $h_{b}=15 \mathrm{~m}$ )

1 Single tier co-channel interfering model $\left(f_{c}=900 \mathrm{MHz}\right)$ [77]

2 Two tier co-channel interfering model $\left(f_{c}=900 \mathrm{MHz}\right)$

3 Single tier co-channel interfering model $\left(f_{c}=2 \mathrm{GHz}\right)[77]$

4 Two tier co-channel interfering model $\left(f_{c}=2 \mathrm{GHz}\right)$

5 Single tier co-channel interfering model $\left(f_{c}=3.35 \mathrm{GHz}\right)$ [77]

6 Two tier co-channel interfering model $\left(f_{c}=3.35 \mathrm{GHz}\right)$

7 Single tier co-channel interfering model $\left(f_{c}=8.45 \mathrm{GHz}\right)[77]$

8 Two tier co-channel interfering model $\left(f_{c}=8.45 \mathrm{GHz}\right)$

9 Single tier co-channel interfering model $\left(f_{c}=15.75 \mathrm{GHz}\right)$ [77]

10 Two tier co-channel interfering model $\left(f_{c}=15.75 \mathrm{GHz}\right)$ 


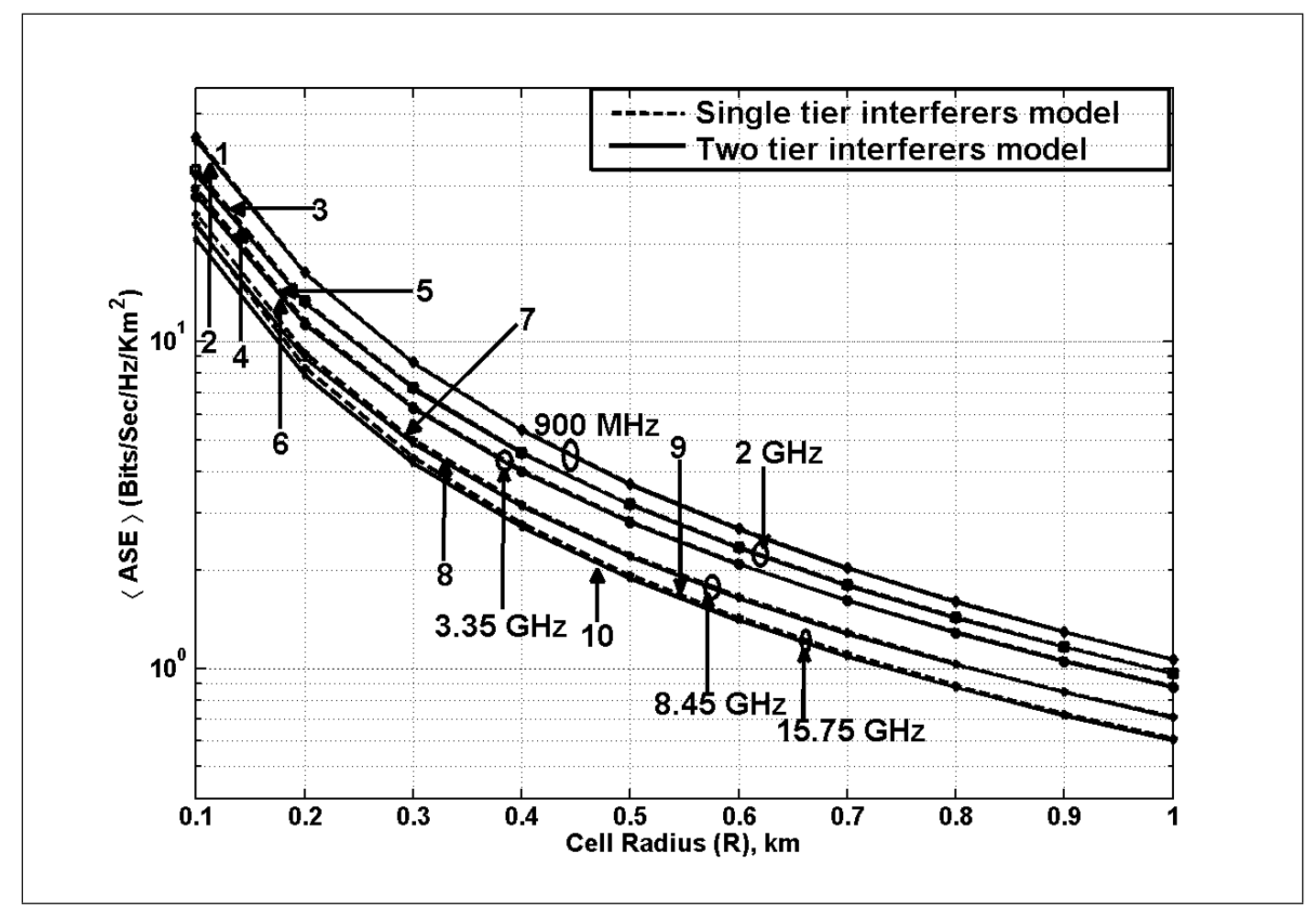

Figure 4.12: Average uplink area spectral efficiency versus cell size radius for path loss exponent, $\alpha=3$, and different carrier frequencies $f_{c}$. (Fully-loaded system with first and second tier co-channel interfering cells, $N_{F I}=6$ and $N_{S I}=12$; extra path loss exponent, $\rho=4$; MS and BS antenna heights : $h_{m}=1.5 \mathrm{~m}$ and $h_{b}=15 \mathrm{~m}$ )

1 Single tier co-channel interfering model $\left(f_{c}=900 \mathrm{MHz}\right)$ [77]

2 Two tier co-channel interfering model $\left(f_{c}=900 \mathrm{MHz}\right)$

3 Single tier co-channel interfering model $\left(f_{c}=2 \mathrm{GHz}\right)$ [77]

4 Two tier co-channel interfering model $\left(f_{c}=2 \mathrm{GHz}\right)$

5 Single tier co-channel interfering model $\left(f_{c}=3.35 \mathrm{GHz}\right)[77]$

6 Two tier co-channel interfering model $\left(f_{c}=3.35 \mathrm{GHz}\right)$

7 Single tier co-channel interfering model $\left(f_{c}=8.45 \mathrm{GHz}\right)[77]$

8 Two tier co-channel interfering model $\left(f_{c}=8.45 \mathrm{GHz}\right)$

9 Single tier co-channel interfering model $\left(f_{c}=15.75 \mathrm{GHz}\right)$ [77]

10 Two tier co-channel interfering model $\left(f_{c}=15.75 \mathrm{GHz}\right)$ 


\begin{tabular}{|l|l|l|l|}
\hline \multicolumn{5}{|c|}{$\mathrm{h}_{m}=1.5 \mathrm{~m}, \mathrm{~h}_{b}=15 \mathrm{~m}$ and $\rho=4$} \\
\hline$\alpha$ & $f_{c}$ & $R[\mathrm{~m}]$ & Percentage Decrease (\%) \\
\hline \multirow{5}{*}{2} & $900 \mathrm{MHz}$ & 100 & 4.56 \\
& $2 \mathrm{GHz}$ & 100 & 8 \\
& $3.35 \mathrm{GHz}$ & 100 & 11.13 \\
& $8.45 \mathrm{GHz}$ & 100 & 17.23 \\
& $15.75 \mathrm{GHz}$ & 100 & 20.57 \\
\hline \multirow{5}{*}{2.5} & $900 \mathrm{MHz}$ & 100 & 3.22 \\
& $2 \mathrm{GHz}$ & 100 & 5.45 \\
& $3.35 \mathrm{GHz}$ & 100 & 7.57 \\
& $8.45 \mathrm{GHz}$ & 100 & 11.80 \\
& $15.75 \mathrm{GHz}$ & 100 & 14.23 \\
\hline \multirow{5}{*}{2.8} & $900 \mathrm{MHz}$ & 100 & 2.61 \\
& $2 \mathrm{GHz}$ & 100 & 4.34 \\
& $3.35 \mathrm{GHz}$ & 100 & 6.01 \\
& $8.45 \mathrm{GHz}$ & 100 & 9.38 \\
& $15.75 \mathrm{GHz}$ & 100 & 11.29 \\
\hline \multirow{5}{*}{3} & $900 \mathrm{MHz}$ & 100 & 2.27 \\
& $2 \mathrm{GHz}$ & 100 & 3.77 \\
& $3.35 \mathrm{GHz}$ & 100 & 5.17 \\
& $8.45 \mathrm{GHz}$ & 100 & 8.06 \\
& $15.75 \mathrm{GHz}$ & 100 & 9.67 \\
\hline
\end{tabular}

Table 4.3: Percentage Decrease in Area Spectral Efficiency between single and two tier Interference Model for Different Basic Path loss Exponent and Cell Size Radius, $R=100$ $\mathrm{m}$.

that for a TDMA-based cellular system operating at higher carrier frequencies as cell size radius reduces and basic path loss becomes smaller second tier co-channel interfering cells become active. Table 4.3 and 4.4, shows summary of the result for $R=100$ and $300 \mathrm{~m}$. 


\begin{tabular}{|l|l|l|l|}
\hline \multicolumn{3}{|c|}{$\mathrm{h}_{m}=1.5 \mathrm{~m}, \mathrm{~h}_{b}=15 \mathrm{~m}$ and $\rho=4$} \\
\hline$\alpha$ & $f_{c}$ & $R[\mathrm{~m}]$ & Percentage Decrease (\%) \\
\hline \multirow{5}{*}{2} & $900 \mathrm{MHz}$ & 300 & 1.23 \\
& $2 \mathrm{GHz}$ & 300 & 2 \\
& $3.35 \mathrm{GHz}$ & 300 & 3 \\
& $8.45 \mathrm{GHz}$ & 300 & 6.46 \\
& $15.75 \mathrm{GHz}$ & 300 & 9.86 \\
\hline \multirow{5}{*}{2.5} & $900 \mathrm{MHz}$ & 300 & 0.83 \\
& $2 \mathrm{GHz}$ & 300 & 1.31 \\
& $3.35 \mathrm{GHz}$ & 300 & 1.1 .92 \\
& $8.45 \mathrm{GHz}$ & 300 & 4.00 \\
& $15.75 \mathrm{GHz}$ & 300 & 6.10 \\
\hline \multirow{5}{*}{2.8} & $900 \mathrm{MHz}$ & 300 & 0.67 \\
& $2 \mathrm{GHz}$ & 300 & 1.02 \\
& $3.35 \mathrm{GHz}$ & 300 & 1.48 \\
& $8.45 \mathrm{GHz}$ & 300 & 3.05 \\
& $15.75 \mathrm{GHz}$ & 300 & 4.6 \\
\hline \multirow{5}{*}{3} & $900 \mathrm{MHz}$ & 300 & 0.57 \\
& $2 \mathrm{GHz}$ & 300 & 0.87 \\
& $3.35 \mathrm{GHz}$ & 300 & 1.25 \\
& $8.45 \mathrm{GHz}$ & 300 & 2.54 \\
& $15.75 \mathrm{GHz}$ & 300 & 3.83 \\
\hline
\end{tabular}

Table 4.4: Percentage Decrease in Area Spectral Efficiency between single and two tier Interference Model for Different Basic Path loss Exponent and Cell Size Radius, $R=300$ m.

\subsubsection{Effect of Extra Path Loss Exponent-Two Tier Interference}

This section presents results on the impact of extra path loss exponent on the information capacity of cellular wireless network. Figures $4.13-4.16$, illustrate the effect of extra path loss exponent $\rho$ on the information capacity performance of cellular wireless system operating at higher carrier frequencies and smaller cell size radii, when both first and second tier co-channel interfering cells are active. The curves show that as $f_{c}$ increases 
from 0.9 to $15.75 \mathrm{GHz}$, and cell size radius decreases from 1000 - $100 \mathrm{~m}$, the $A S E$ increases exponentially, the figure confirms that $A S E$ is an increasing function of both $\rho$ and $R$.

Figure 4.13, shows that, for extra path loss exponent $\rho=2$ and carrier frequencies $f_{c}=0.9,2$ and $3.35 \mathrm{GHz}$, there is a decrease in $A S E$, between $4.86-17.21 \%$ from $R<$ $300 \mathrm{~m}$. However, for $f_{c}=8.45$, and $15.75 \mathrm{GHz}$, when $R \leq 500 \mathrm{~m}$ the decrease in $A S E$ between our proposed model and the conventional model was between $8.645-23.22 \%$. An interesting observation is that for $R=400 \mathrm{~m}$ the $A S E$ for our proposed interference model for $f_{c}=8.45 \mathrm{GHz}$ is the same as the $A S E$ for the conventional interference model at 15.75 GHz. This confirms the importance of the consideration of second tier co-channel interference in cellular system information capacity performance analysis at higher carrier frequencies and smaller cell size radii. Figure 4.14, shows that, for $\rho=3$ and $f_{c}=0.9,2$ and $3.35 \mathrm{GHz}$, the curves of the $A S E$, for the two interference models overlay each other. However, for $f_{c}=8.45$, and $15.75 \mathrm{GHz}$, when $R \leq 500 \mathrm{~m}$ the decrease in ASE between the proposed model and conventional were between $5-18 \%$, and $21.74-7.36 \%$.

Figure 4.15, shows the curves when $\rho=5$. The figure illustrates that for carrier frequencies $f_{c}=0.9,2$ and $3.35 \mathrm{GHz}$, the curves for the two interference models overlay. For $f_{c}=8.45 \mathrm{GHz}$ the curves overlay from $R \geq 400$. However, the curves show a decrease in $A S E$ of between $4.80-15.51 \%$ for $100 \leq R \leq 300 \mathrm{~m}$. In the case of $15.75 \mathrm{GHz}$, the curves overlay from $500-1000 \mathrm{~m}$. For cell size radius $R$ between $100-500 \mathrm{~m}$ the decrease in $A S E$ between the two interference models is between $6.1-19.38 \%$. The curves in Figure 4.16, illustrate the information capacity performance for the two interference models, for extra path loss exponent $\rho=8$. The figure, shows that for carrier frequencies $f_{c}=0.9,2$ and 3.35, the curves overlay. For $8.45 \mathrm{GHz}$ the curves overlay between $\mathrm{R}=$ $300-1000$, and decrease in $A S E$ is between $4.56-11.15 \%$ for $R \leq 300 \mathrm{~m}$. The graph, illustrates that for a carrier frequency $f_{c}=15.75 \mathrm{GHz}$, the curves for the conventional and proposed interference model overlay each other from cell size radius $R=1000 \mathrm{~m}$ down to $R=300 \mathrm{~m}$. For $100 \leq R \leq 300 \mathrm{~m}$ the decrease in $A S E$ between the two models is between $4.56-16.1 \%$. Figure 4.15 and 4.16, reveals that as $\rho$ increases the second tier co-channel interference becomes less severe. This contributes to decrease in ASE between the two models becoming minimal. Table 4.5 and 4.6, present a summary the result for $R=100$ and $300 \mathrm{~m}$. 


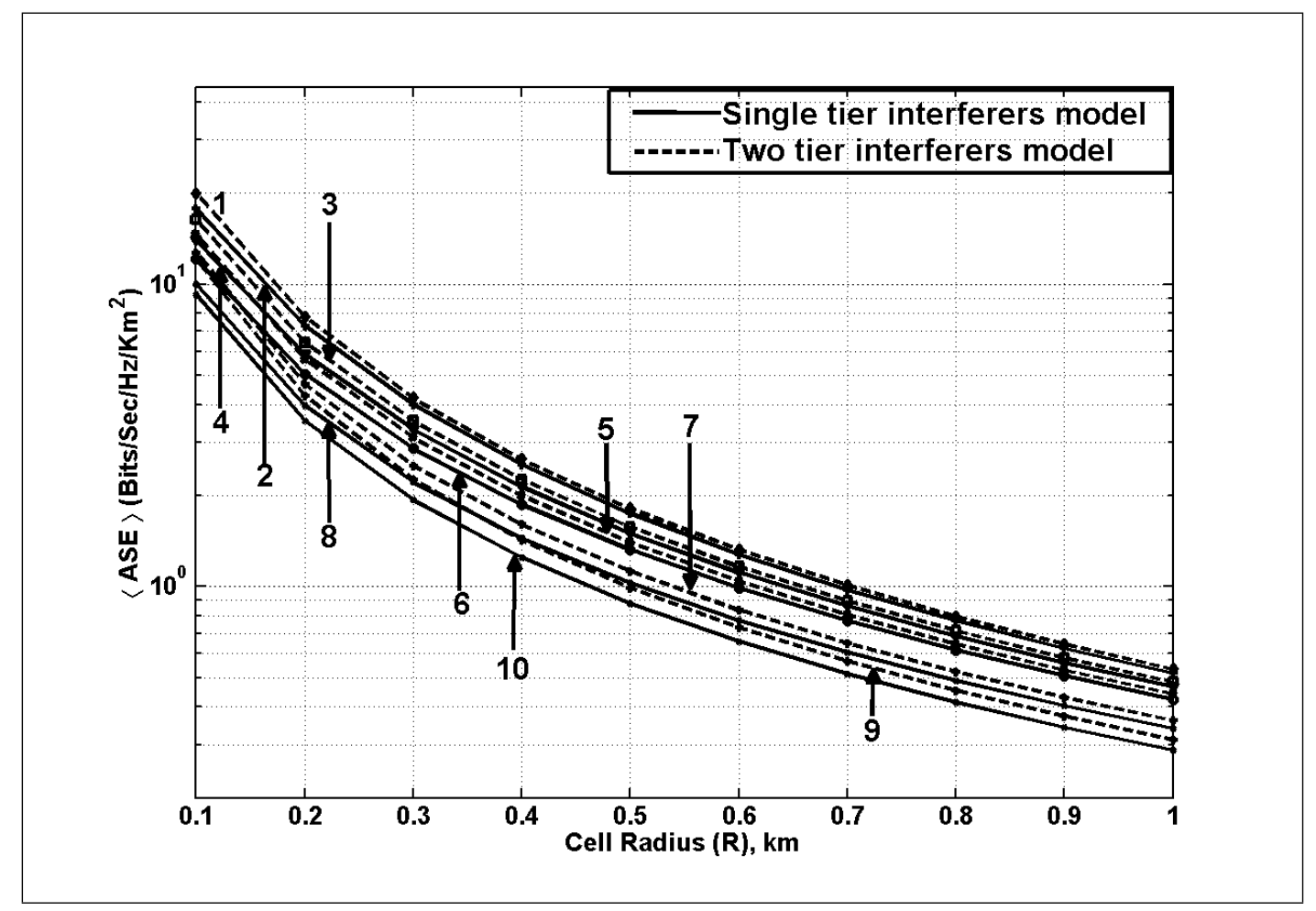

Figure 4.13: Average uplink area spectral efficiency versus cell size radius for extra path loss exponent, $\rho=2$, and different carrier frequencies, $f_{c}$. (Fully-loaded system with first and second tier co-channel interfering cells, $N_{F I}=6$ and $N_{S I}=12$; path loss exponent, $\alpha$ $=2 ; \mathrm{MS}$ and BS antenna heights $: h_{m}=1.5 \mathrm{~m}$ and $h_{b}=15 \mathrm{~m}$ )

1 Single tier co-channel interfering model $\left(f_{c}=900 \mathrm{MHz}\right)$ [77]

2 Two tier co-channel interfering model $\left(f_{c}=900 \mathrm{MHz}\right)$

3 Single tier co-channel interfering model $\left(f_{c}=2 \mathrm{GHz}\right)$ [77]

4 Two tier co-channel interfering model $\left(f_{c}=2 \mathrm{GHz}\right)$

5 Single tier co-channel interfering model $\left(f_{c}=3.35 \mathrm{GHz}\right)$ [77]

6 Two tier co-channel interfering model $\left(f_{c}=3.35 \mathrm{GHz}\right)$

7 Single tier co-channel interfering model $\left(f_{c}=8.45 \mathrm{GHz}\right)$ [77]

8 Two tier co-channel interfering model $\left(f_{c}=8.45 \mathrm{GHz}\right)$

9 Single tier co-channel interfering model $\left(f_{c}=15.75 \mathrm{GHz}\right)$ [77]

10 Two tier co-channel interfering model $\left(f_{c}=15.75 \mathrm{GHz}\right)$ 


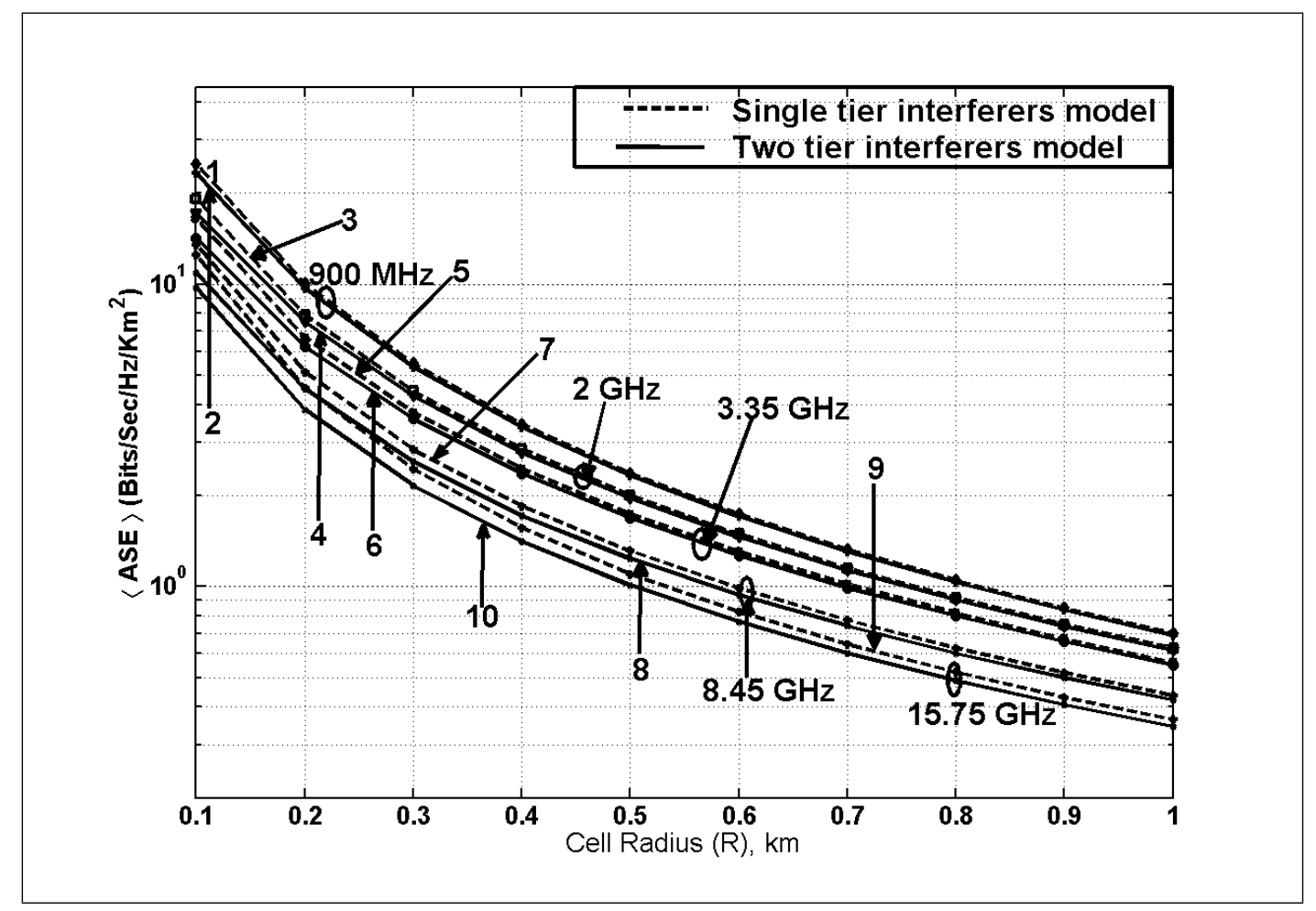

Figure 4.14: Average uplink area spectral efficiency versus cell size radius for extra path loss exponent, $\rho=3$, and different carrier frequencies, $f_{c}$. (Fully-loaded system with first and second tier co-channel interfering cells, $N_{F I}=6$ and $N_{S I}=12$; path loss exponent, $\alpha$ $=2 ; \mathrm{MS}$ and BS antenna heights $: h_{m}=1.5 \mathrm{~m}$ and $h_{b}=15 \mathrm{~m}$ )

1 Single tier co-channel interfering model $\left(f_{c}=900 \mathrm{MHz}\right)$ [77]

2 Two tier co-channel interfering model $\left(f_{c}=900 \mathrm{MHz}\right)$

3 Single tier co-channel interfering model $\left(f_{c}=2 \mathrm{GHz}\right)$ [77]

4 Two tier co-channel interfering model $\left(f_{c}=2 \mathrm{GHz}\right)$

5 Single tier co-channel interfering model $\left(f_{c}=3.35 \mathrm{GHz}\right)$ [77]

6 Two tier co-channel interfering model $\left(f_{c}=3.35 \mathrm{GHz}\right)$

7 Single tier co-channel interfering model $\left(f_{c}=8.45 \mathrm{GHz}\right)$ [77]

8 Two tier co-channel interfering model $\left(f_{c}=8.45 \mathrm{GHz}\right)$

9 Single tier co-channel interfering model $\left(f_{c}=15.75 \mathrm{GHz}\right)$ [77]

10 Two tier co-channel interfering model $\left(f_{c}=15.75 \mathrm{GHz}\right)$ 


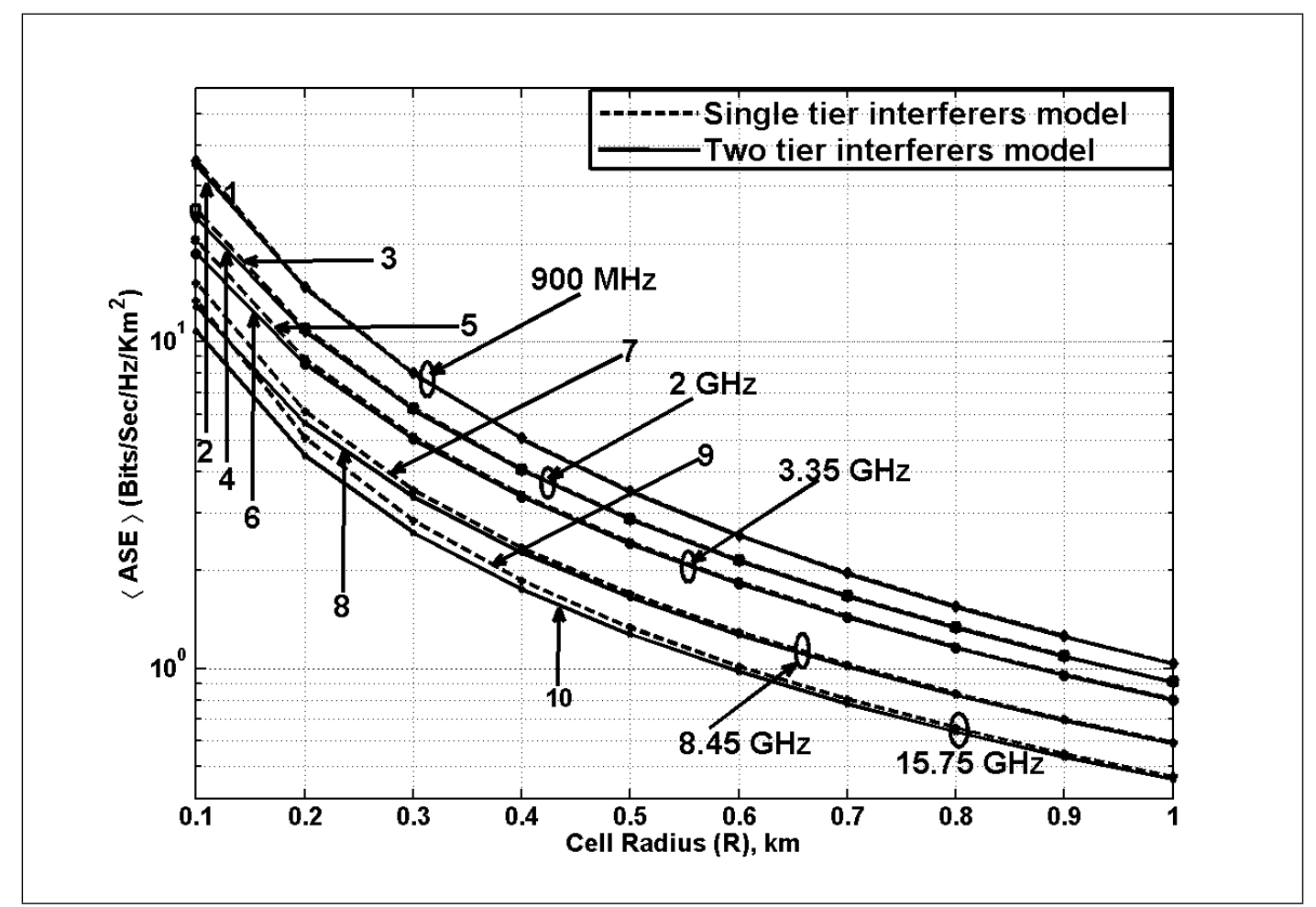

Figure 4.15: Average uplink area spectral efficiency versus cell size radius for extra path loss exponent, $\rho=5$, and different carrier frequencies, $f_{c}$. (Fully-loaded system with first and second tier co-channel interfering cells, $N_{F I}=6$ and $N_{S I}=12$; path loss exponent, $\alpha$ $=2 ; \mathrm{MS}$ and BS antenna heights $: h_{m}=1.5 \mathrm{~m}$ and $h_{b}=15 \mathrm{~m}$ )

1 Single tier co-channel interfering model $\left(f_{c}=900 \mathrm{MHz}\right)$ [77]

2 Two tier co-channel interfering model $\left(f_{c}=900 \mathrm{MHz}\right)$

3 Single tier co-channel interfering model $\left(f_{c}=2 \mathrm{GHz}\right)[77]$

4 Two tier co-channel interfering model $\left(f_{c}=2 \mathrm{GHz}\right)$

5 Single tier co-channel interfering model $\left(f_{c}=3.35 \mathrm{GHz}\right)$ [77]

6 Two tier co-channel interfering model $\left(f_{c}=3.35 \mathrm{GHz}\right)$

7 Single tier co-channel interfering model $\left(f_{c}=8.45 \mathrm{GHz}\right)$ [77]

8 Two tier co-channel interfering model $\left(f_{c}=8.45 \mathrm{GHz}\right)$

9 Single tier co-channel interfering model $\left(f_{c}=15.75 \mathrm{GHz}\right)$ [77]

10 Two tier co-channel interfering model $\left(f_{c}=15.75 \mathrm{GHz}\right)$ 


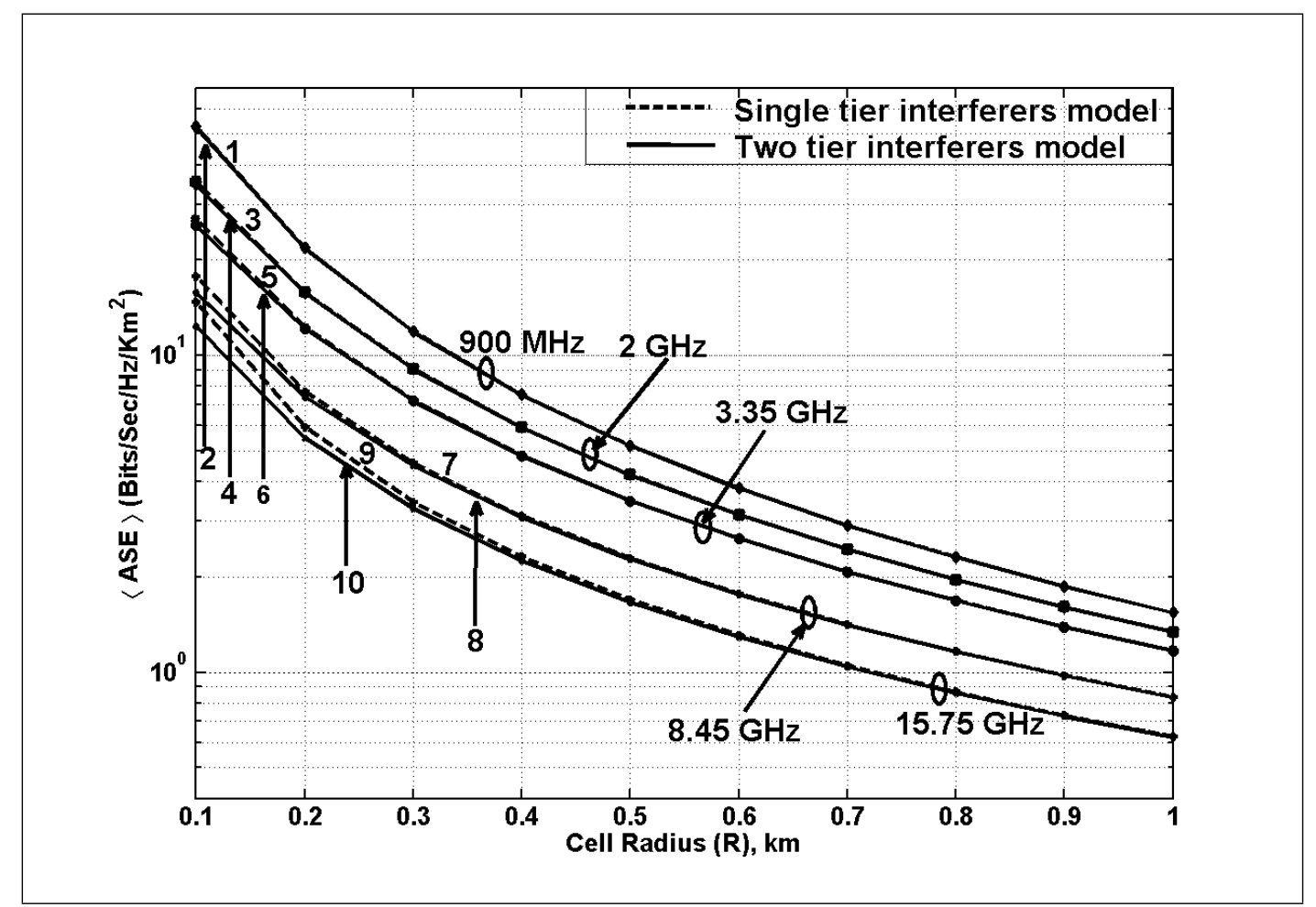

Figure 4.16: Average uplink area spectral efficiency versus cell size radius for extra path loss exponent, $\rho=8$, and different carrier frequencies, $f_{c}$. (Fully-loaded system with first and second tier co-channel interfering cells, $N_{F I}=6$ and $N_{S I}=12$; path loss exponent, $\alpha$ $=2 ; \mathrm{MS}$ and BS antenna heights $: h_{m}=1.5 \mathrm{~m}$ and $h_{b}=15 \mathrm{~m}$ )

1 Single tier co-channel interfering model $\left(f_{c}=900 \mathrm{MHz}\right)$ [77]

2 Two tier co-channel interfering model $\left(f_{c}=900 \mathrm{MHz}\right)$

3 Single tier co-channel interfering model $\left(f_{c}=2 \mathrm{GHz}\right)[77]$

4 Two tier co-channel interfering model $\left(f_{c}=2 \mathrm{GHz}\right)$

5 Single tier co-channel interfering model $\left(f_{c}=3.35 \mathrm{GHz}\right)[77]$

6 Two tier co-channel interfering model $\left(f_{c}=3.35 \mathrm{GHz}\right)$

7 Single tier co-channel interfering model $\left(f_{c}=8.45 \mathrm{GHz}\right)[77]$

8 Two tier co-channel interfering model $\left(f_{c}=8.45 \mathrm{GHz}\right)$

9 Single tier co-channel interfering model $\left(f_{c}=15.75 \mathrm{GHz}\right)$ [77]

10 Two tier co-channel interfering model $\left(f_{c}=15.75 \mathrm{GHz}\right)$ 


\begin{tabular}{|c|c|c|c|}
\hline \multicolumn{4}{|c|}{$\mathrm{h}_{m}=1.5 \mathrm{~m}, \mathrm{~h}_{b}=15 \mathrm{~m}$ and $\alpha=2$} \\
\hline$\rho$ & $f_{c}$ & $R[\mathrm{~m}]$ & Percentage Decrease $(\%)$ \\
\hline \multirow{5}{*}{2} & $900 \mathrm{MHz}$ & 100 & 11.06 \\
\hline & $2 \mathrm{GHz}$ & 100 & 14.6 \\
\hline & $3.35 \mathrm{GHz}$ & 100 & 17.21 \\
\hline & $8.45 \mathrm{GHz}$ & 100 & 21.30 \\
\hline & $15.75 \mathrm{GHz}$ & 100 & 23.22 \\
\hline \multirow{5}{*}{3} & $900 \mathrm{MHz}$ & 100 & 7.78 \\
\hline & $2 \mathrm{GHz}$ & 100 & 10.76 \\
\hline & $3.35 \mathrm{GHz}$ & 100 & 13.82 \\
\hline & $8.45 \mathrm{GHz}$ & 100 & 19.19 \\
\hline & $15.75 \mathrm{GHz}$ & 100 & 21.85 \\
\hline \multirow{5}{*}{5} & $900 \mathrm{MHz}$ & 100 & 3.0 \\
\hline & $2 \mathrm{GHz}$ & 100 & 5.9 \\
\hline & $3.35 \mathrm{GHz}$ & 100 & 8.93 \\
\hline & $8.45 \mathrm{GHz}$ & 100 & 15.51 \\
\hline & $15.75 \mathrm{GHz}$ & 100 & 19.39 \\
\hline \multirow{5}{*}{8} & $900 \mathrm{MHz}$ & 100 & 1.0 \\
\hline & $2 \mathrm{GHz}$ & 100 & 2.5 \\
\hline & $3.35 \mathrm{GHz}$ & 100 & 4.60 \\
\hline & $8.45 \mathrm{GHz}$ & 100 & 11.15 \\
\hline & $15.75 \mathrm{GHz}$ & 100 & 16.9 \\
\hline
\end{tabular}

Table 4.5: Percentage Decrease in Area Spectral Efficiency between single and two tier Interference Model for Different Extra Path loss Exponent and Cell Size Radius, $R=100$ $\mathrm{m}$. 


\begin{tabular}{|l|l|l|l|}
\hline \multicolumn{4}{|c|}{$\mathrm{h}_{m}=1.5 \mathrm{~m}, \mathrm{~h}_{b}=15 \mathrm{~m}$ and $\alpha=2$} \\
\hline$\rho$ & $f_{c}$ & $R[\mathrm{~m}]$ & Percentage Decrease (\%) \\
\hline \multirow{5}{*}{2} & $900 \mathrm{MHz}$ & 300 & 4.86 \\
& $2 \mathrm{GHz}$ & 300 & 6.43 \\
& $3.35 \mathrm{GHz}$ & 300 & 7.95 \\
& $8.45 \mathrm{GHz}$ & 300 & 11.77 \\
& $15.75 \mathrm{GHz}$ & 300 & 14.46 \\
\hline \multirow{5}{*}{3} & $900 \mathrm{MHz}$ & 300 & 2.40 \\
& $2 \mathrm{GHz}$ & 300 & 3.55 \\
& $3.35 \mathrm{GHz}$ & 300 & 4.86 \\
& $8.45 \mathrm{GHz}$ & 300 & 11.99 \\
& $15.75 \mathrm{GHz}$ & 300 & 15.33 \\
\hline \multirow{5}{*}{5} & $900 \mathrm{MHz}$ & 300 & 0.66 \\
& $2 \mathrm{GHz}$ & 300 & 1.77 \\
& $3.35 \mathrm{GHz}$ & 300 & 1.87 \\
& $8.45 \mathrm{GHz}$ & 300 & 4.81 \\
& $15.75 \mathrm{GHz}$ & 300 & 8.15 \\
\hline & $900 \mathrm{MHz}$ & 300 & 0.17 \\
& $2 \mathrm{GHz}$ & 300 & 0.25 \\
& $8.45 \mathrm{GHz}$ & 300 & 0.48 \\
& $15.75 \mathrm{GHz}$ & 300 & 1.99 \\
\hline
\end{tabular}

Table 4.6: Percentage Decrease in Area Spectral Efficiency between single and two tier Interference Model for Different Extra Path loss Exponent and Cell Size Radius, $R=300$ m.

\subsubsection{Effect of Base Station Antenna Height-Two Tier Interference}

A comparison of Figures 4.13, 4.17 and 4.18, shows the effect of BS antenna height $h_{b}$ on the information capacity performance, at different carrier frequencies, for the conventional and proposed interference model. The plot shows that when BS antenna height $h_{b}=15$ $\mathrm{m}$, basic path loss exponent $\alpha=2$, and extra path loss exponent $\rho=2$, for $f_{c}=0.9,2$ and $3.35 \mathrm{GHz}$, the decrease in ASE is between $4.9-17.21 \%$ for $R \leq 300 \mathrm{~m}$. For 


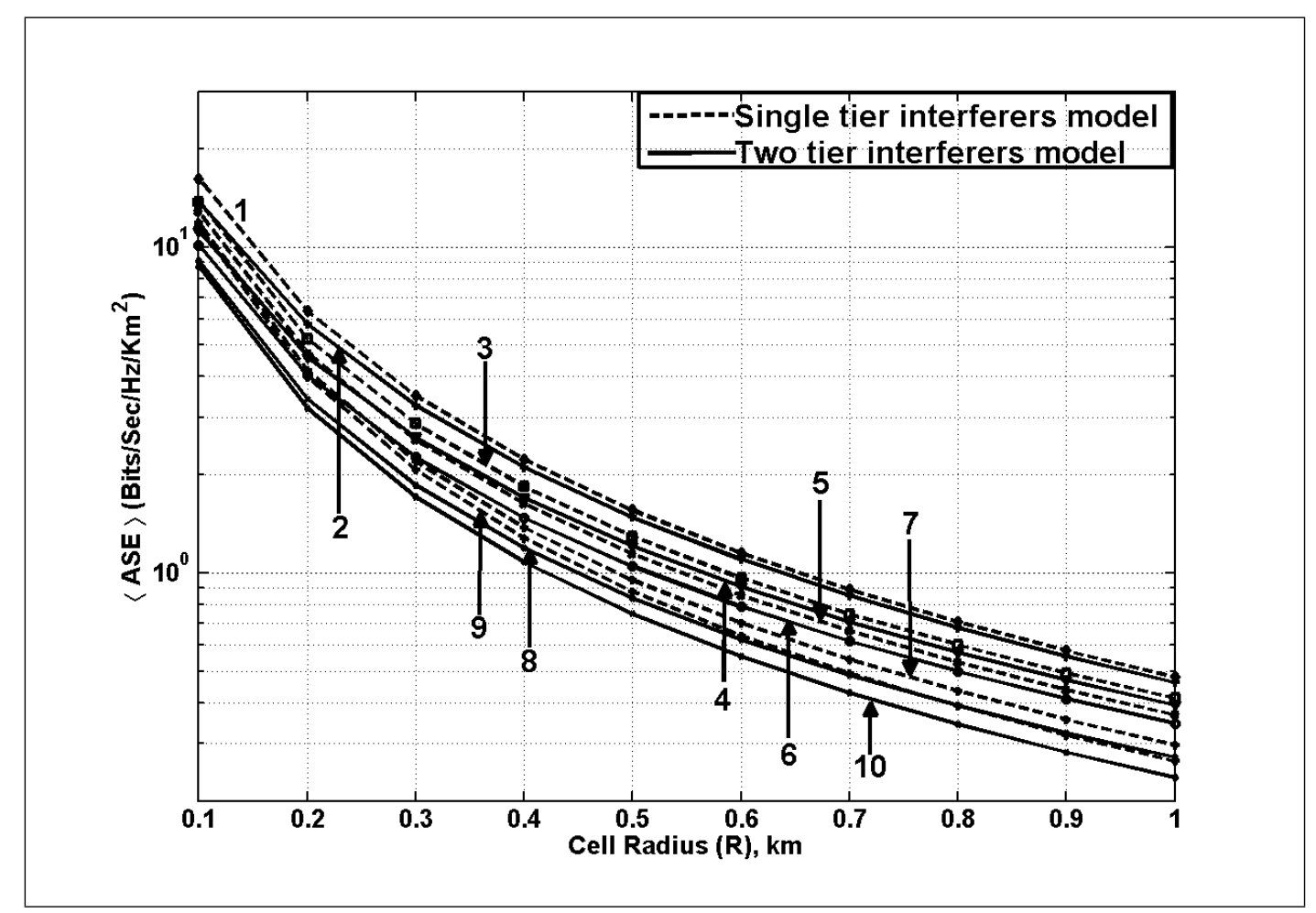

Figure 4.17: Average uplink area spectral efficiency versus cell size radius for BS station antenna height $h_{b}=35 \mathrm{~m}$, and different carrier frequencies, $f_{c}$. (Fully-loaded cellular system with co-channel interfering cells in first and second tier, $N_{F I}=6$, and $N_{S I}=12$; path loss exponent, $\alpha=2$, extra path loss exponent, $\rho=2$; MS antenna heights : $h_{m}=1.5$ $\mathrm{m})$

1 Single tier co-channel interfering model $\left(f_{c}=900 \mathrm{MHz}\right)$ [77]

2 Two tier co-channel interfering model $\left(f_{c}=900 \mathrm{MHz}\right)$

3 Single tier co-channel interfering model $\left(f_{c}=2 \mathrm{GHz}\right)[77]$

4 Two tier co-channel interfering model $\left(f_{c}=2 \mathrm{GHz}\right)$

5 Single tier co-channel interfering model $\left(f_{c}=3.35 \mathrm{GHz}\right)$ [77]

6 Two tier co-channel interfering model $\left(f_{c}=3.35 \mathrm{GHz}\right)$

7 Single tier co-channel interfering model $\left(f_{c}=8.45 \mathrm{GHz}\right)$ [77]

8 Two tier co-channel interfering model $\left(f_{c}=8.45 \mathrm{GHz}\right)$

9 Single tier co-channel interfering model $\left(f_{c}=15.75 \mathrm{GHz}\right)[77]$

10 Two tier co-channel interfering model $\left(f_{c}=15.75 \mathrm{GHz}\right)$ 


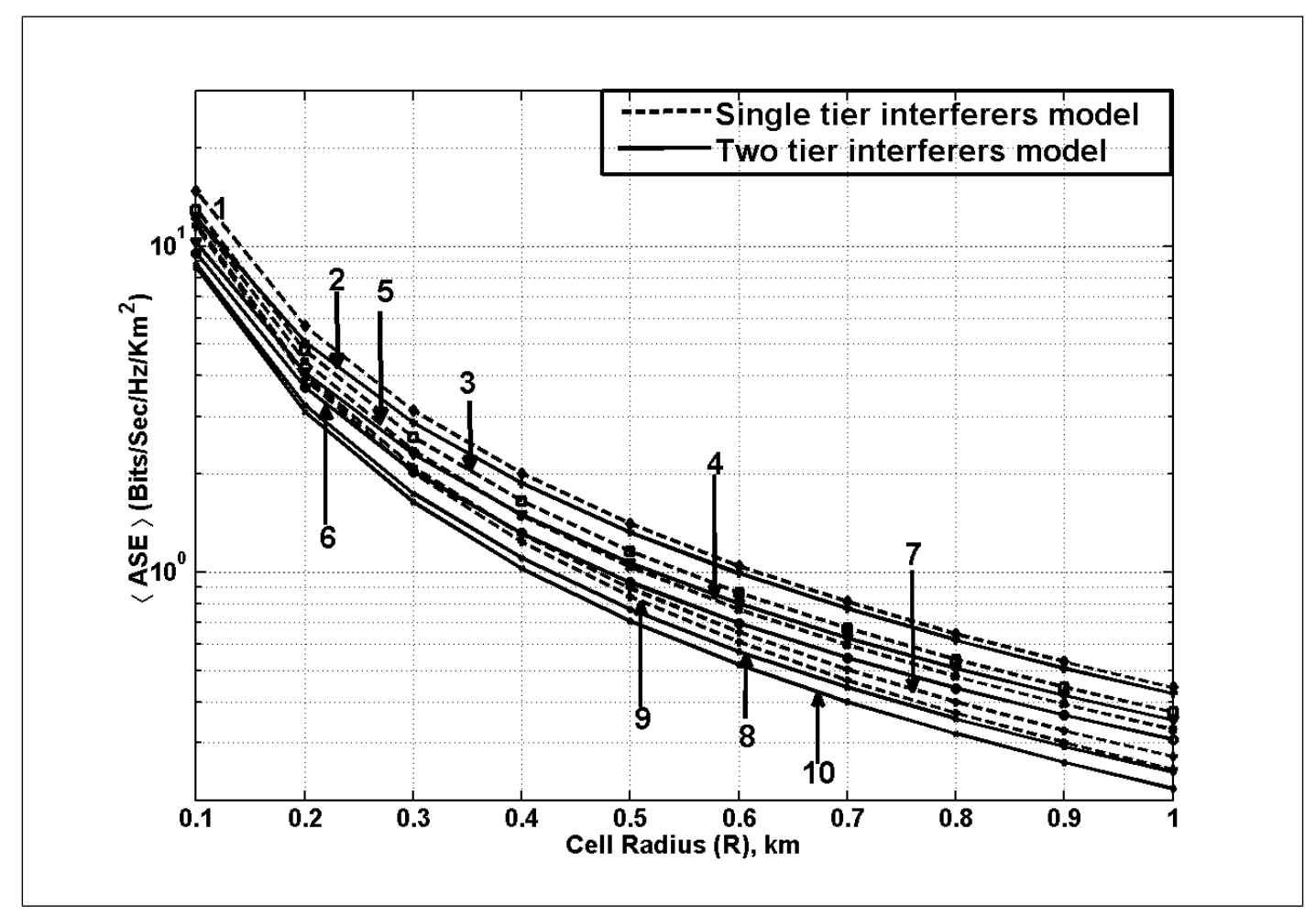

Figure 4.18: Average uplink area spectral efficiency versus cell size radius for BS station antenna height $h_{b}=55 \mathrm{~m}$, and different carrier frequencies, $f_{c}$. (Fully-loaded cellular system with co-channel interfering cells in first and second tier, $N_{F I}=6$, and $N_{S I}=12$; path loss exponent, $\alpha=2$, extra path loss exponent, $\rho=2$; MS antenna heights : $h_{m}=1.5$ $\mathrm{m})$

1 Single tier co-channel interfering model $\left(f_{c}=900 \mathrm{MHz}\right)$ [77]

2 Two tier co-channel interfering model $\left(f_{c}=900 \mathrm{MHz}\right)$

3 Single tier co-channel interfering model $\left(f_{c}=2 \mathrm{GHz}\right)[77]$

4 Two tier co-channel interfering model $\left(f_{c}=2 \mathrm{GHz}\right)$

5 Single tier co-channel interfering model $\left(f_{c}=3.35 \mathrm{GHz}\right)$ [77]

6 Two tier co-channel interfering model $\left(f_{c}=3.35 \mathrm{GHz}\right)$

7 Single tier co-channel interfering model $\left(f_{c}=8.45 \mathrm{GHz}\right)$ [77]

8 Two tier co-channel interfering model $\left(f_{c}=8.45 \mathrm{GHz}\right)$

9 Single tier co-channel interfering model $\left(f_{c}=15.75 \mathrm{GHz}\right)[77]$

10 Two tier co-channel interfering model $\left(f_{c}=15.75 \mathrm{GHz}\right)$ 
$f_{c}=8.45$ and $15.75 \mathrm{GHz}$, the decrease in $A S E$ between the two interference models increases as the cell size radius $R$ decreases. Figure 4.17 and 4.18 , shows that as the BS antenna height and carrier frequency increases, the decrease in $A S E$ between the two interference models also increases. This is, because as $h_{b}$ increases the breakpoint distance $g$ increases, causing the second tier interfering cell to be in the region before $g$. This makes the second tier co-channel interfering cells to become more severe.

\subsubsection{Effect of Vehicular Traffic-Two Tier Interference}

Figure 4.19 - 4.22, illustrates the effect of the effective road height, $h$ on $A S E$ at carrier frequencies greater than $2 \mathrm{GHz}$, when first and second tier co-channel interfering cells are active. The figures show that previous results still hold. The figures show that the decrease in $A S E$ between the conventional and proposed interference model increases as the carrier frequency increases and cell size radius reduces. The figures illustrate that the decrease in $A S E$ is lesser for heavy vehicular traffic as compared to light vehicular traffic. For the case of light vehicular traffic at $R=100 \mathrm{~m}$, for $f_{c}=3.35,8.45$ and $15.75 \mathrm{GHz}$ the decrease in $A S E$ is $17.18,21.22$ and $23.18 \%$, whilst for heavy vehicular traffic it is $11.63,16.00$ and $19.00 \%$. We therefore, conclude that at microwave carrier frequencies greater than $2 \mathrm{GHz}$ and smaller cell size radius the effect of second tier co-channel interference is minimal during heavy vehicular traffic (day time), relative to light vehicular traffic (night time). This is because the breakpoint distance during day time is shorter as compared to night time, which is due to the increment of the effective road height [refer to equation (3.9)]. The figures also show that increasing the base station antenna height, $h_{b}$ leads to second tier co-channel interference becoming more severe. The figure, also reveals that even if $h_{b}$ is increased, the decrease in $A S E$ between the two interference model is still less for day time as compared to the night time. 


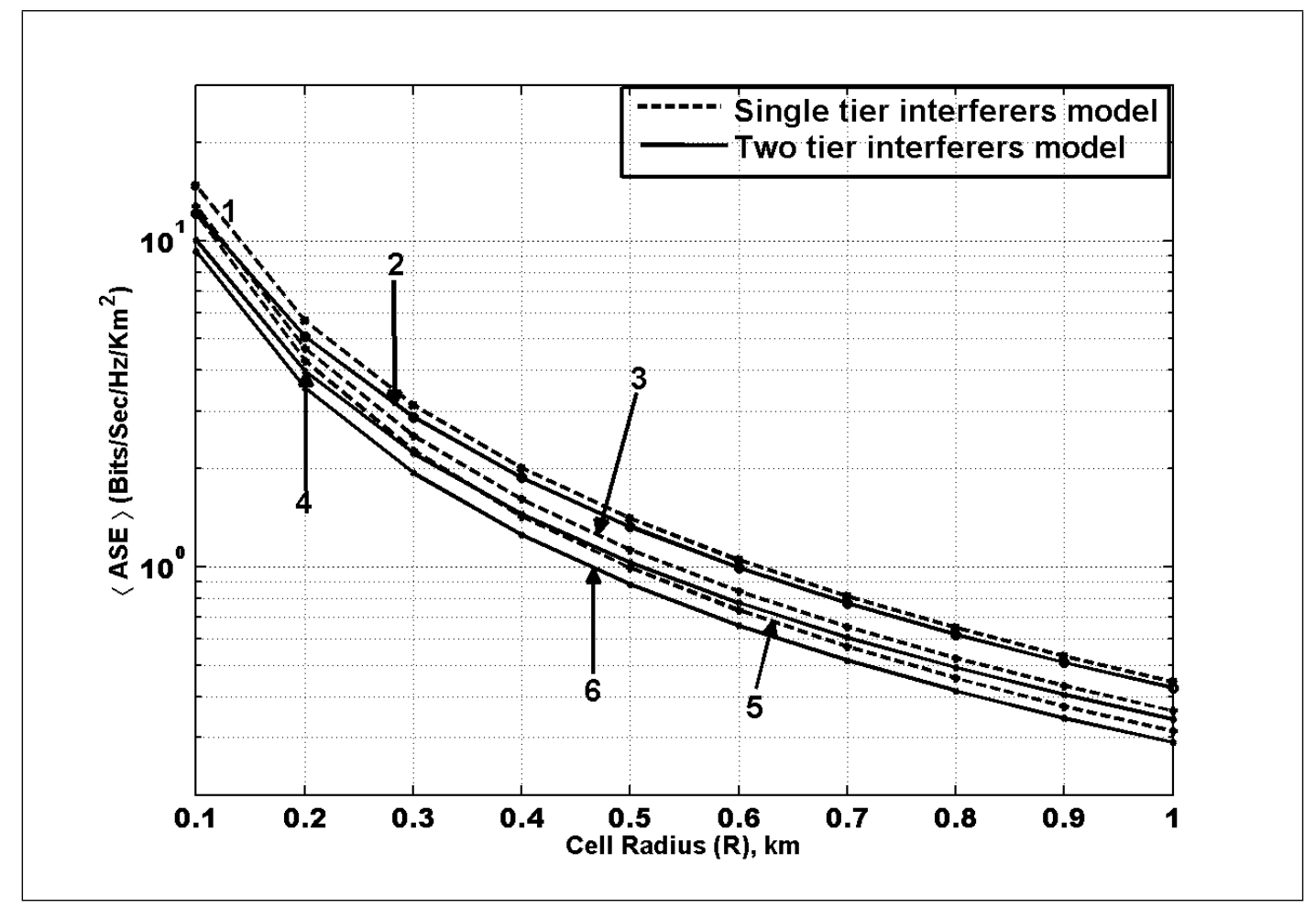

Figure 4.19: Average uplink area spectral efficiency versus cell size radius for light vehicular traffic with effective road height and BS antenna height, $h=0.3 \mathrm{~m}$ and $h_{b}=15 \mathrm{~m}$, for different carrier frequencies, $f_{c}$. (Fully-loaded cellular system with co-channel interfering cells in first and second tier, $N_{F I}=6$, and $N_{S I}=12$; path loss exponent, $\alpha=2$ and extra path loss exponent $\rho=2$; MS antenna height, $h_{m}=1.8 \mathrm{~m}$ )

1 Single tier co-channel interfering model $\left(f_{c}=3.35 \mathrm{GHz}\right)$

2 Two tier co-channel interfering model $\left(f_{c}=3.35 \mathrm{GHz}\right)$

3 Single tier co-channel interfering model $\left(f_{c}=8.45 \mathrm{GHz}\right)$

4 Two tier co-channel interfering model $\left(f_{c}=8.45 \mathrm{GHz}\right)$

5 Single tier co-channel interfering model $\left(f_{c}=15.75 \mathrm{GHz}\right)$

6 Two tier co-channel interfering model $\left(f_{c}=15.75 \mathrm{GHz}\right)$ 


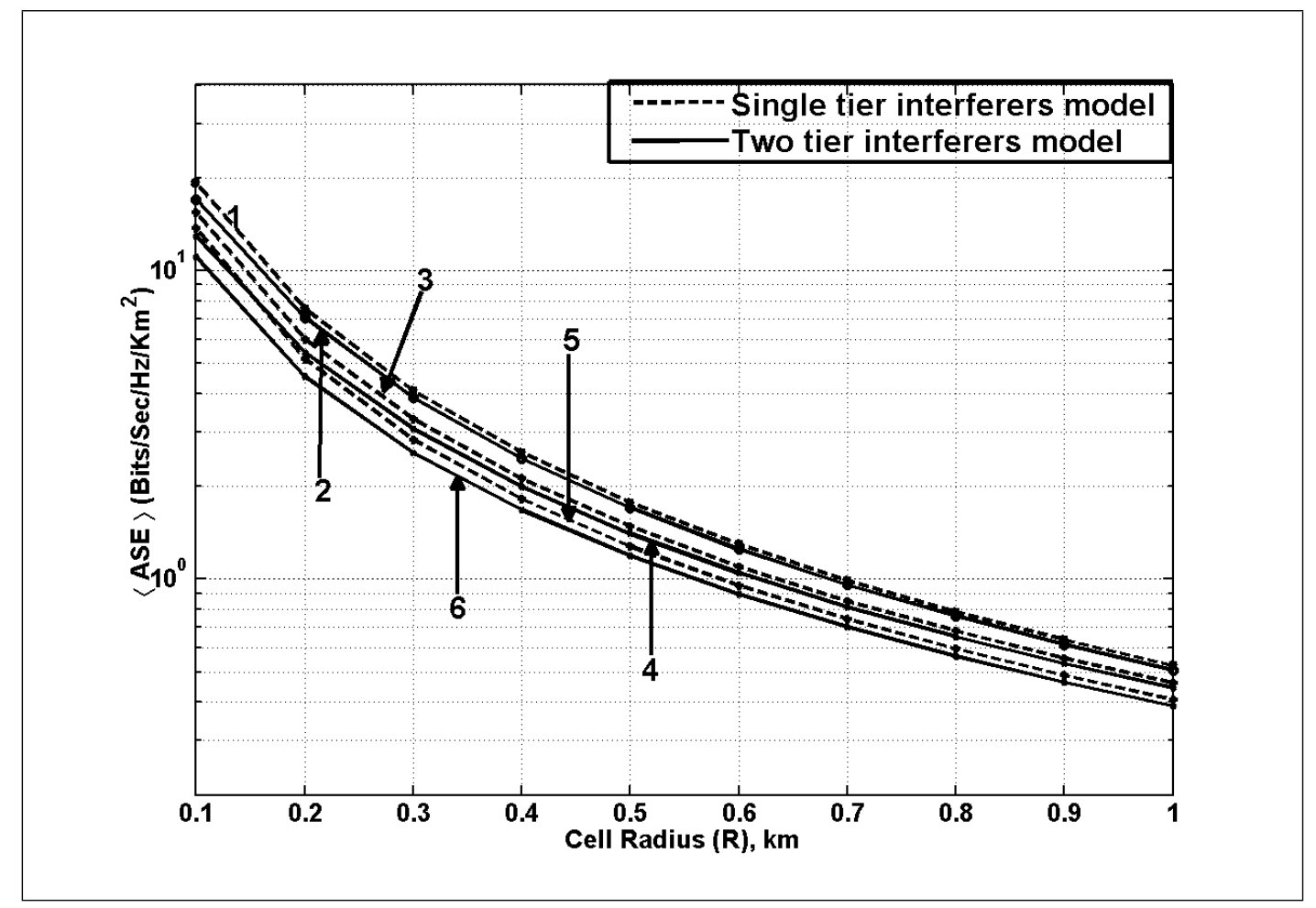

Figure 4.20: Average uplink area spectral efficiency versus cell size radius for heavy vehicular traffic with effective road height and BS antenna height $h=1.29 \mathrm{~m}$ and $h_{b}=$ $15 \mathrm{~m}$, for different carrier frequencies, $f_{c}$. (Fully-loaded cellular system with co-channel interfering cells in first and second tier, $N_{F I}=6$, and $N_{S I}=12$; path loss exponent, $\alpha=2$ and extra path loss exponent $\rho=2$; MS antenna height, $h_{m}=1.8 \mathrm{~m}$ )

1 Single tier co-channel interfering model $\left(f_{c}=3.35 \mathrm{GHz}\right)$

2 Two tier co-channel interfering model $\left(f_{c}=3.35 \mathrm{GHz}\right)$

3 Single tier co-channel interfering model $\left(f_{c}=8.45 \mathrm{GHz}\right)$

4 Two tier co-channel interfering model $\left(f_{c}=8.45 \mathrm{GHz}\right)$

5 Single tier co-channel interfering model $\left(f_{c}=15.75 \mathrm{GHz}\right)$

6 Two tier co-channel interfering model $\left(f_{c}=15.75 \mathrm{GHz}\right)$ 


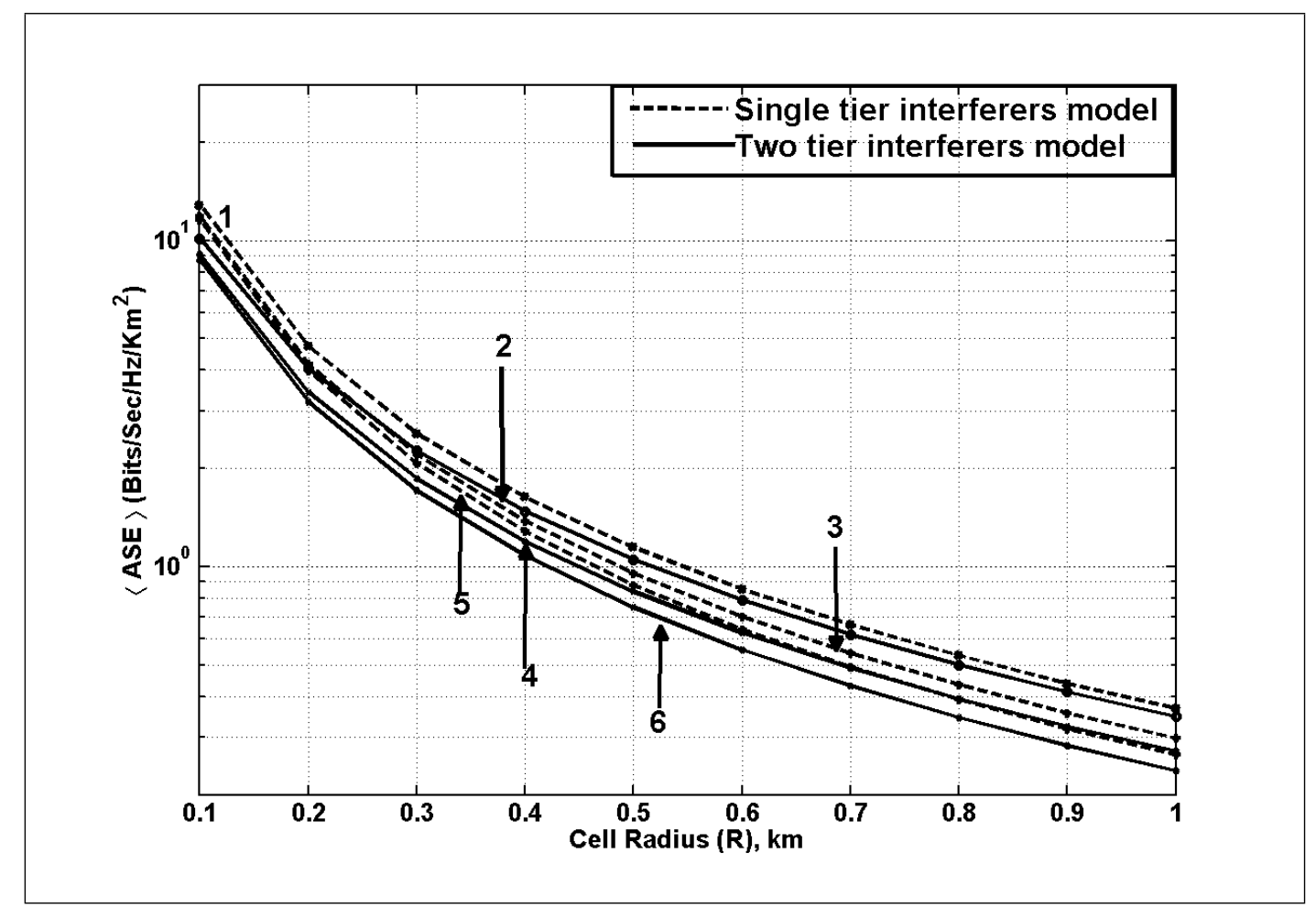

Figure 4.21: Average uplink area spectral efficiency versus cell size radius for light vehicular traffic with effective road height and BS antenna height, $h=0.3 \mathrm{~m}$ and $h_{b}=35 \mathrm{~m}$, for different carrier frequencies, $f_{c}$. (Fully-loaded cellular system with co-channel interfering cells in first and second tier, $N_{F I}=6$, and $N_{S I}=12$; path loss exponent, $\alpha=2$ and extra path loss exponent $\rho=2$; MS antenna height, $h_{m}=1.8 \mathrm{~m}$ )

1 Single tier co-channel interfering model $\left(f_{c}=3.35 \mathrm{GHz}\right)$

2 Two tier co-channel interfering model $\left(f_{c}=3.35 \mathrm{GHz}\right)$

3 Single tier co-channel interfering model $\left(f_{c}=8.45 \mathrm{GHz}\right)$

4 Two tier co-channel interfering model $\left(f_{c}=8.45 \mathrm{GHz}\right)$

5 Single tier co-channel interfering model $\left(f_{c}=15.75 \mathrm{GHz}\right)$

6 Two tier co-channel interfering model $\left(f_{c}=15.75 \mathrm{GHz}\right)$ 


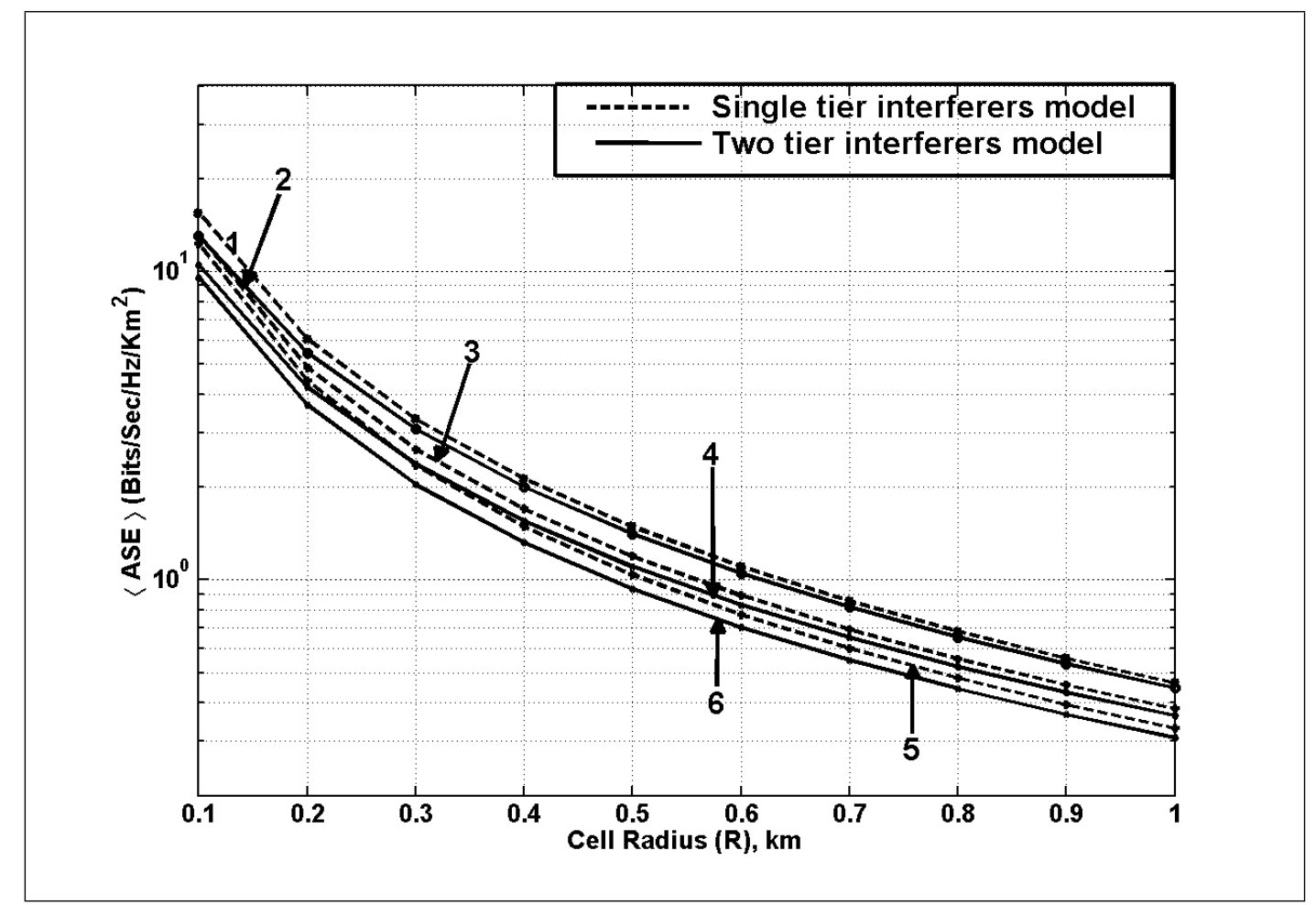

Figure 4.22: Average uplink area spectral efficiency versus cell size radius for heavy vehicular traffic with effective road height and BS antenna height, $h=1.29 \mathrm{~m}$ and $h_{b}=$ $35 \mathrm{~m}$, for different carrier frequencies, $f_{c}$. (Fully-loaded cellular system with co-channel interfering cells in first and second tier, $N_{F I}=6$, and $N_{S I}=12$; path loss exponent, $\alpha=2$ and extra path loss exponent $\rho=2$; MS antenna height, $h_{m}=1.8 \mathrm{~m}$ )

1 Single tier co-channel interfering model $\left(f_{c}=3.35 \mathrm{GHz}\right)$

2 Two tier co-channel interfering model $\left(f_{c}=3.35 \mathrm{GHz}\right)$

3 Single tier co-channel interfering model $\left(f_{c}=8.45 \mathrm{GHz}\right)$

4 Two tier co-channel interfering model $\left(f_{c}=8.45 \mathrm{GHz}\right)$

5 Single tier co-channel interfering model $\left(f_{c}=15.75 \mathrm{GHz}\right)$

6 Two tier co-channel interfering model $\left(f_{c}=15.75 \mathrm{GHz}\right)$ 


\subsection{Summary}

Building upon the work presented in chapter 3, in this chapter using the proposed cochannel interference model we mathematically characterized the area spectral efficiency $A S E$ as a function of normalized reuse distance and cell size radius, for information capacity performance analysis. Comparing the $A S E$ which incorporates our proposed cochannel interference model with an existing $A S E$ of cellular mobile radio systems [77]; simulation results show that as carrier frequency increases and cell size radius decreases the $A S E$ of a cellular wireless decrease. The information capacity performance analysis results also suggested that at higher microwave carrier frequencies and smaller cell size radii second tier co-channel interfering cells become active. Because co-channel interference is the major limiting factor that affect the information capacity performance of cellular wireless systems [3]. The results of our study show the need to incorporate the second tier co-channel interfering cells in the information capacity analysis of emerging and future wireless systems.

We also studied the impact of propagation loss and system parameters on the information capacity performance of cellular wireless communication system. The study was for cellular system operating at higher microwave carrier frequencies greater than 2 $\mathrm{GHz}$ and smaller cell size radii, when both the first and second tier co-channel interfering cells are active. It was shown that at higher carrier frequencies and smaller cell size radius the information capacity of a cellular wireless system was affected by propagation loss and system parameters. Now, the case of including other tiers (multiple tiers) apart from the first and second tiers and studying, (i) the information capacity performance, (ii) the effect of system and propagation loss parameters on the information capacity, will be an important extension of this chapter. In the next chapter, a multiple tier co-channel interference model is proposed, and we study the information capacity of the cellular system, when multiple tiers of co-channel interfering cells are active. The impact of propagation loss and system parameters on the information capacity performance of cellular wireless, when multiple tiers of co-channel interfering cells are active is also studied. 


\section{Chapter 5}

\section{Proposed Multiple Tier Co-channel \\ Interference Model and Information}

Capacity Performance Analysis for

Multiple Tier Co-channel Interference 


\subsection{Introduction}

In the previous chapter, a two tier co-channel interference model was proposed for emerging and future cellular wireless communication systems. It was shown that for emerging and future cellular wireless communication systems, second tier co-channel interference become active, as cell size radius decreases and carrier frequency increases. A study of the impact of system and propagation loss parameters on information capacity performance of cellular system model was also performed.

In this chapter, we propose a co-channel interference model which includes multiple tiers of co-channel interfering cells. Mathematical analysis, supported by computer simulation is used to show that at higher microwave carrier frequencies and smaller cell size radii, multiple tiers of co-channel interfering cell becomes active. Our analysis is based on the uplink information capacity performance of a cellular wireless communication system, operating at higher microwave carrier frequencies greater than $2 \mathrm{GHz}$ and small cell size radius $(1-1000 \mathrm{~m})$. We then study the impact of both propagation loss and system parameters on the information capacity performance of the cellular wireless communication system, when multiple tiers of co-channel interference are active. Results show that the system information capacity is still sensitive to propagation loss and system parameters as the cell size radius decreases and carrier frequency increases.

The information capacity performance of cellular wireless communication networks is an important issue, for efficient management of the limited radio spectrum. The greatest improvement of the cellular network information capacity has come from cell size reduction, by installing more base stations $[39,113]$. For the successful implementation of a high-speed data service it is imperative that we use design and planning tools, which can accurately predict the information capacity of cellular wireless networks. This depends on a fundamental understanding of the signal propagation mechanism, resulting channel characteristics, interference level, and the effect of system parameters. Unlike conventional wireless networks emerging and future wireless networks will be operating at microwave carrier frequencies greater than $2 \mathrm{GHz}$, to enable wireless communication systems provide high-speed data service [85]. Multi-antenna can also improve the system capacity of cellular wireless communication system proportionally with the minimum 
number of antennas at the transmitter and receiver in a single cell wireless communication system [114]. However, in multiple cell wireless communication systems the system information capacity is degraded by co-channel interference even with multiple antennas at the transmitters and receivers $[115,116]$. Therefore, co-channel interference (CCI) modelling and information capacity analysis are of great importance in the next generation cellular wireless communications systems. However, most research work still uses previous co-channel interference models for information capacity performance analysis, for next generation cellular wireless communication systems, which will be operating at higher carrier frequencies and smaller cell size radii.

In [101], Ahmed et al., studied the capacity and interference statistics of highways W-CDMA microcellular wireless system operating in the UHF band, without considering multiple tiers of co-channel interference. Ahmed et al., in their work introduced a model for cigar-shaped cells in rural zones. They used the two-slope path loss model with a general propagation exponent to investigate the interference statistics of the uplink and the sector capacity.

Hernández-Valdez et al., [28], studied the impact of both propagation and system parameters on the performance of microcellular networks. They considered cellular systems operating at higher carrier frequencies well above the $2 \mathrm{GHz}$ band used by thirdgeneration wireless systems. However, outage probability was used for the study and multiple tiers of co-channel interfering cells was not considered. Anang et al., in [46], studied the sensitivity of cellular system information capacity performance to BS antenna height. The study was for land mobile cellular systems operating at microwave frequencies beyond $2 \mathrm{GHz}$. The evaluation was done in terms of the information capacity of the cellular wireless network, when both the first 6 , and second 12 , co-channel interfering cells were active, without considering multiple tiers of co-channel interfering cells. The recent work by Anang et al., [47], has taken an initial step to include second tier co-channel interference in the study of the impact of propagation loss parameters on the uplink information capacity of a cellular wireless network, operating at higher microwave carrier frequencies and smaller cell radii. However, Anang et al., did not consider multiple tiers of co-channel interference.

In previous studies [88], Singh et al., proposed a model for uplink interference with 
power control and cell selection. In the model only first and second tier co-channel interfering cells were considered to be active. Ge et al. in [79], also proposed a co-channel interference model for multi-cell wireless communication systems. However, the model and capacity analysis were for multiple-input multiple-output (MIMO) cellular wireless systems. Xiaohu et al. in [79], reported that co-channel interference modelling and capacity analysis is of great importance in the next generation cellular wireless communication systems, because of the higher carrier frequencies.

Although numerous co-channel interference models have been proposed most are based on the first and second tier co-channel interfering cells and are models for lower carrier frequencies. Likewise, extensive research work has been carried out on the effect of propagation loss and system parameters on the information capacity of cellular wireless communication system for design and planning purposes. However, most of this work, did not consider multiple tiers of co-channel interference. In this chapter the following contribution is made:

- We propose a co-channel interference model, which includes multiple tier of cochannel interfering cells. It is shown that as carrier frequency increases and cell size radius reduces, multiple tiers of co-channel interfering cell become active.

- We provide an analysis on the impact of system parameters (such as cell size, antenna height, operating frequency, and reuse factor), on the information capacity performance of emerging cellular wireless systems, when multiple tier of co-channel interfering cells are active.

- We study the impact of propagation loss parameters (such as breakpoint, effective road height, and path loss exponent), on the information capacity performance of emerging cellular wireless networks operating at carrier frequencies greater than 2 $\mathrm{GHz}$, when multiple tier interferers are active.

The remainder of this chapter is organized as follows: Chapter 5.2, provides general system and channel models for cellular wireless communication systems. It shows the relationship between cell size radius, frequency, breakpoint and the number of active cochannel interfering cells. Chapter 5.3, presents our proposed multiple tier co-channel interference model and outlines the basic assumptions used in the modelling. Chapter 5.4, 
provides expression for the area spectral efficiency $(A S E)$, for a time division multipleaccess (TDMA) cellular system. In Chapter 5.5, the impact of system/propagation parameters on the information capacity in the presence of multiple tier interference is studied. The numerical simulation setup for propagation/system parameter impact analysis is presented in Chapter 5.6. Chapter 5.7, compares the information capacity performance of a cellular wireless system which incorporates the existing (conventional) interference model to a cellular wireless system which incorporates the proposed multiple tier interference model. Computer simulation is used for the comparison. Finally, a summary is presented in Chapter 5.8. The work in this chapter has been published in $[50,117,118]$.

\subsection{Multiple Tier Interference General System Model}

As stated in the previous chapters, wireless channels are characterized by the following physical phenomena: (1) mean path loss, (2) shadowing (slow fading). and (3) small scale fading (fast fading). However, as stated earlier 'snapshot' measurement results are still valid for information capacity analysis. We consider the distance dependent path loss wireless channel as described in Chapter 3.3.1, for our proposed multiple tier interference model. In the quest to facilitate reading of this thesis, we copy the mathematical description of the propagation channel from Chapter 3.3.1. Let $K$ be the constant path loss factor, and let $r$ be the distance between BS and MS. The basic path loss exponent is represented by $\alpha$ and the extra path loss exponent is represented by $\rho$. Notice that power transmitted from a transmitting antenna is for now only affected by path loss and it is represented by $P_{t}$. It must be noted that the channel model presented in this work has been experimentally validated for a smaller cell size radius environment, and for both UHF, and super high frequency (SHF) band $[72,83,84]$. The received signal $P_{r}$ is mathematically represented as:

$$
P_{r}=\frac{K}{r^{\alpha}(1+r / g)^{\rho}} P_{t},
$$

The $g$ represents the break point distance and is given as $4 h_{b} h_{m} / \lambda_{c}$. As described earlier in Chapter 3.3.1, because $K$ and $P_{t}$ cancel out for simplicity we assume $K=1, P_{t}=1$ without 
loss of generality and define the received signal power as

$$
P_{r}=r^{-\alpha}(1+r / g)^{-\rho} .
$$

In the following section, we describe the relationship between carrier frequency, breakpoint distance and the number of tiers of co-channel interferers.

\subsubsection{Relationship between Breakpoint Distance, Carrier Frequency and Number of Co-channel Tiers}

An explanation is given in this section of (i) the relationship between breakpoint distance, and carrier frequency and (ii) the reason why, at higher microwave carrier frequencies, multiple tiers of co-channel interfering cells may become active. To facilitate the understanding of the relationship between breakpoint distance $g$, carrier frequency $f_{c}$ and number of co-channel interfering tier $N_{n}$, first refer to the mathematical description of the breakpoint distance $g$, equation (3.8) in subsection 3.3.2.

To make the relationship understandable and to express physical meaning, we plot a graph of distance dependent averaged received signal power $P_{r}$ (equation (5.1)), against distance, for carrier frequencies $f_{c} \mathrm{~s}^{\mathrm{\prime}}=0.9,2,3.35$ and 8.45. Figures $5.1-5.4$, shows the individual breakpoints distance $g_{1}, g_{2}, g_{3}$ and $g_{4}$ for the two slope path loss model when carrier frequencies $f_{c} \mathrm{~s}^{\prime}=0.9,2,3.35$ and $8.45 \mathrm{GHz}$. Figure 5.5, shows the combined breakpoint distances; $g_{1}, g_{2}, g_{3}$ and $g_{4}$ from their relative BS, for carrier frequencies $f_{c} \mathrm{~s}$ ' $=0.9,2,3.35$ and $8.45 \mathrm{GHz}$. BS and MS antenna height $h_{b}=15 \mathrm{~m}$, and $h_{m}=1.5 \mathrm{~m}$; these values were used in previous work $[85,108]$. The curves show that as $f_{c}$ increases $g$ becomes longer. As stated earlier before the breakpoint, power falls off in inverse proportion to the square power of distance between transmitter and receiver. After the breakpoint the power falls off in inverse proportion to the fourth power of distance between transmitter and receiver. As $g$ becomes longer other tiers of co-channel interfering cells may be in the same region as those of the first tier co-channel interfering cells, as compared to shorter breakpoint distance $g$. This may cause multiple tiers of co-channel interference to become active, which may lead to an increase in co-channel interference. The purpose of subsequent sections of this chapter is to use mathematical analysis supported by computer simulation, 


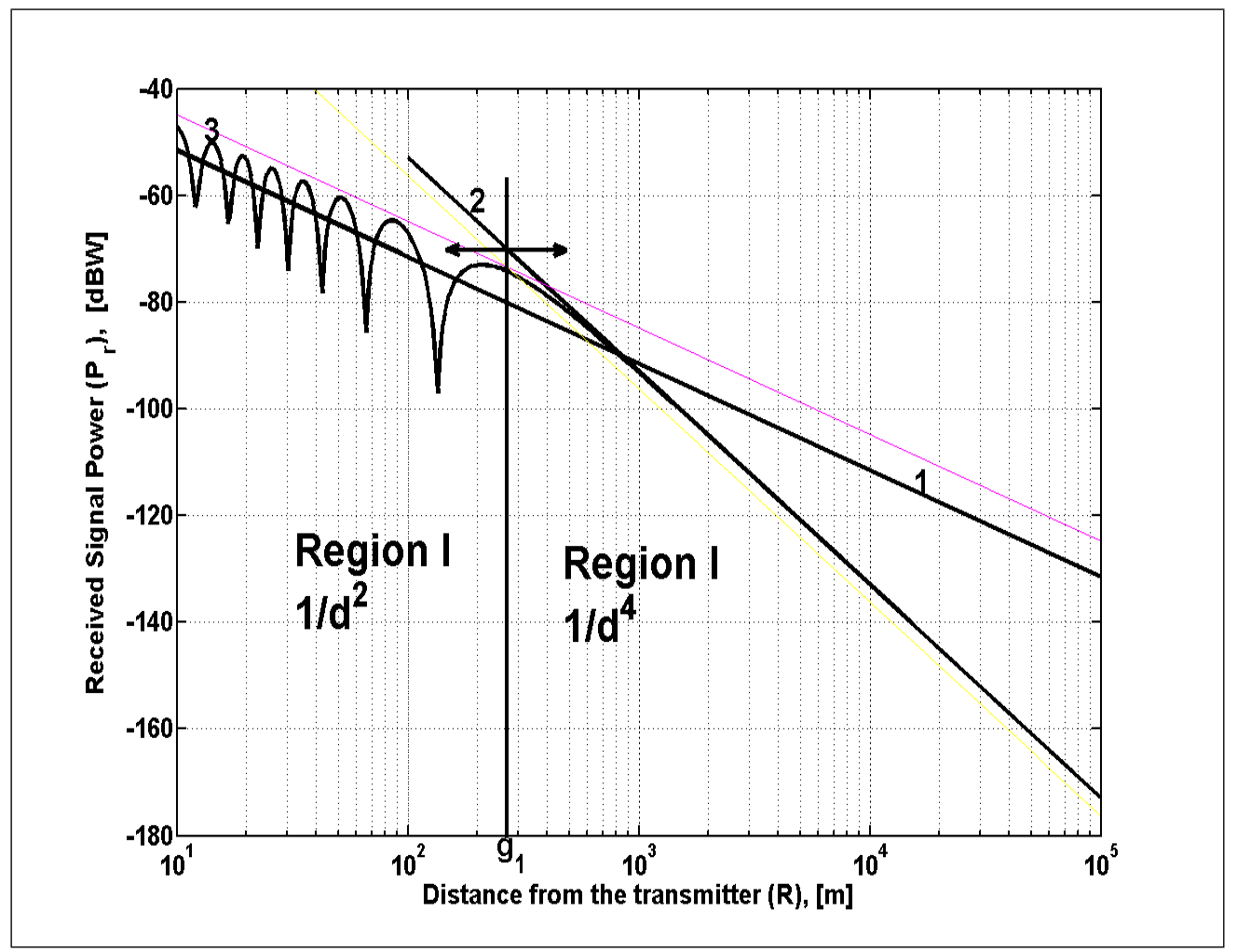

Figure 5.1: Two-slope received signal power, $P_{r}$ versus distance. Showing breakpoint distance $g$ at carrier frequency, $f_{c}=900 \mathrm{MHz}$. (Transmitted power $P_{t}=1 \mathrm{~W}$, MS and BS antenna gain $G_{r}=G_{t}=1$, basic path loss exponent $\alpha=2$, extra path loss exponent $\rho=4$, MS and BS antenna heights: $h_{m}=1.5 \mathrm{~m}$ and $h_{b}=15 \mathrm{~m}$ )

1. Free Space Path Loss [86]

2. Power falls off $\propto 1 /(\text { distance })^{4}$

3. Two Ray path loss [68] 


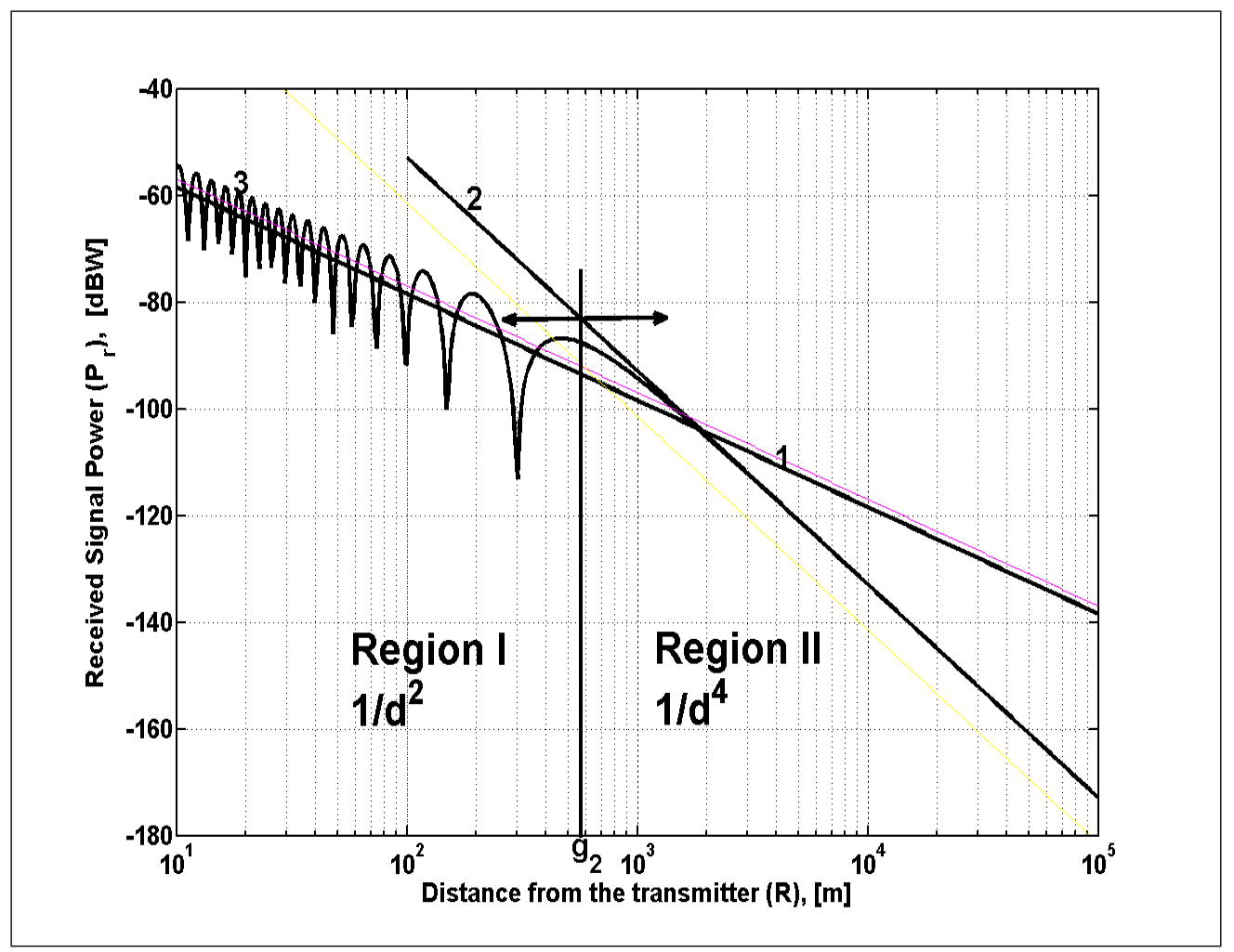

Figure 5.2: Two-slope received signal power, $P_{r}$ versus distance. Showing breakpoint distance $g$ at carrier frequency, $f_{c}=2 \mathrm{GHz}$. (Transmitted power $P_{t}=1 \mathrm{~W}$, MS and BS antenna gain $G_{r}=G_{t}=1$, basic path loss exponent $\alpha=2$, extra path loss exponent $\rho=4$, MS and BS antenna heights: $h_{m}=1.5 \mathrm{~m}$ and $h_{b}=15 \mathrm{~m}$ )

1. Free Space Path Loss [86]

2. Power falls off $\propto 1 /(\text { distance })^{4}$

3. Two Ray path loss [68] 


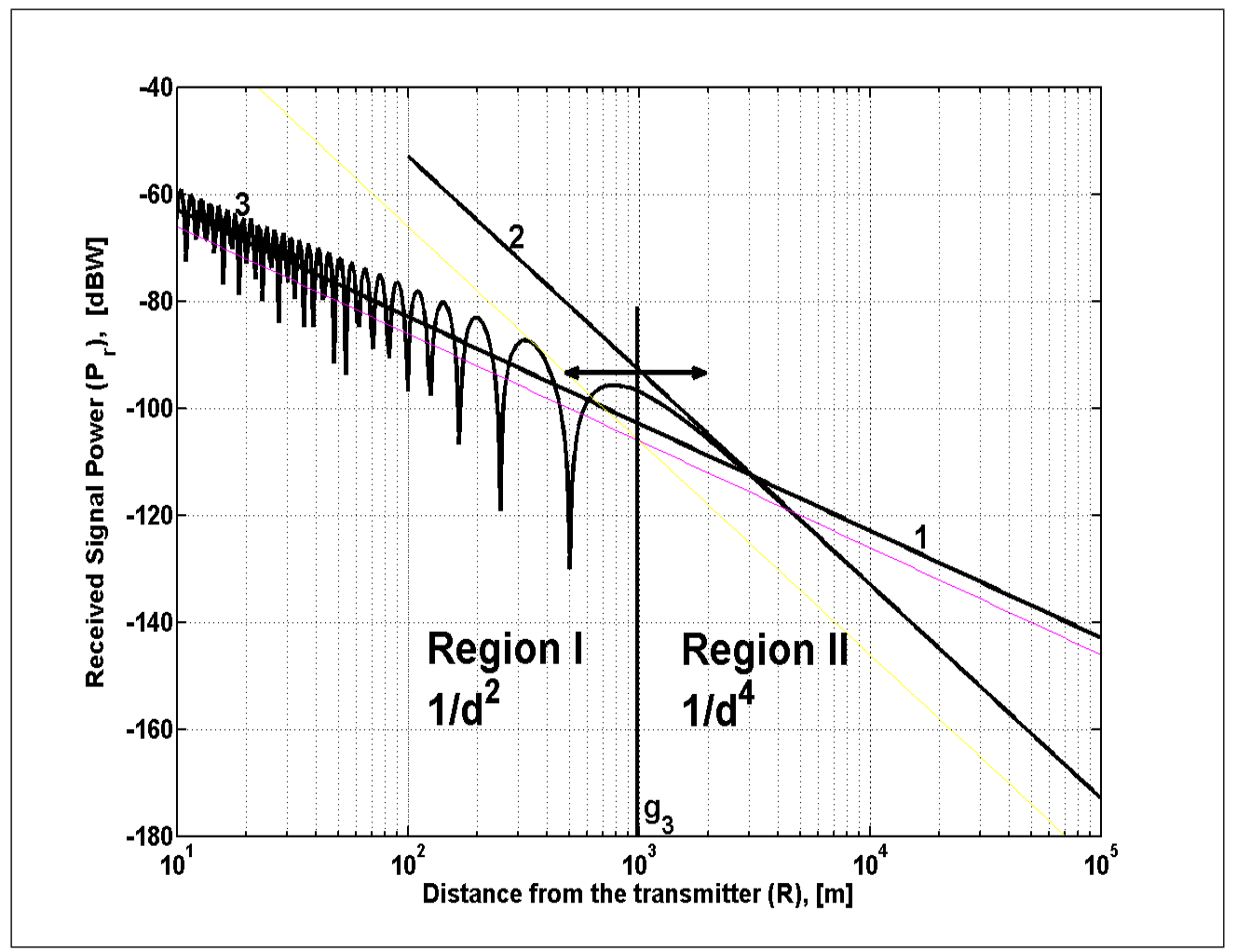

Figure 5.3: Two-slope received signal power, $P_{r}$ versus distance. Showing breakpoint distance $g$ at carrier frequency, $f_{c}=3.35 \mathrm{GHz}$. (Transmitted power $P_{t}=1 \mathrm{~W}$, MS and BS antenna gain $G_{r}=G_{t}=1$, basic path loss exponent $\alpha=2$, extra path loss exponent $\rho=4$, MS and BS antenna heights: $h_{m}=1.5 \mathrm{~m}$ and $h_{b}=15 \mathrm{~m}$ )

1. Free Space Path Loss [86]

2. Power falls off $\propto 1 /(\text { distance })^{4}$

3. Two Ray path loss [68] 


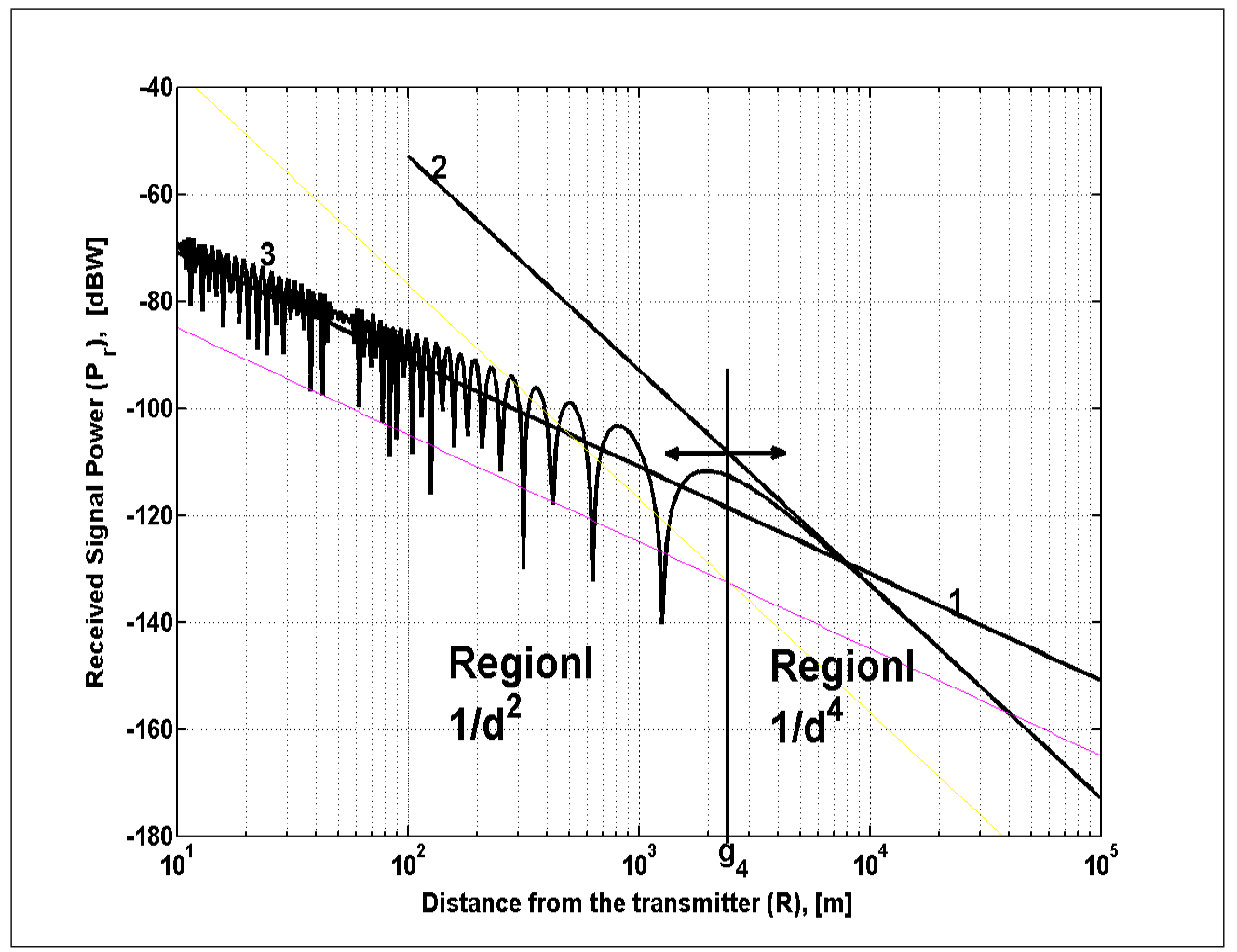

Figure 5.4: Two-slope received signal power, $P_{r}$ versus distance. Showing breakpoint distance $g$ at carrier frequency, $f_{c}=8.45 \mathrm{GHz}$. (Transmitted power $P_{t}=1 \mathrm{~W}$, MS and BS antenna gain $G_{r}=G_{t}=1$, basic path loss exponent $\alpha=2$, extra path loss exponent $\rho=4$, MS and BS antenna heights: $h_{m}=1.5 \mathrm{~m}$ and $h_{b}=15 \mathrm{~m}$ )

1. Free Space Path Loss [86]

2. Power falls off $\propto 1 /(\text { distance })^{4}$

3. Two Ray path loss [68] 


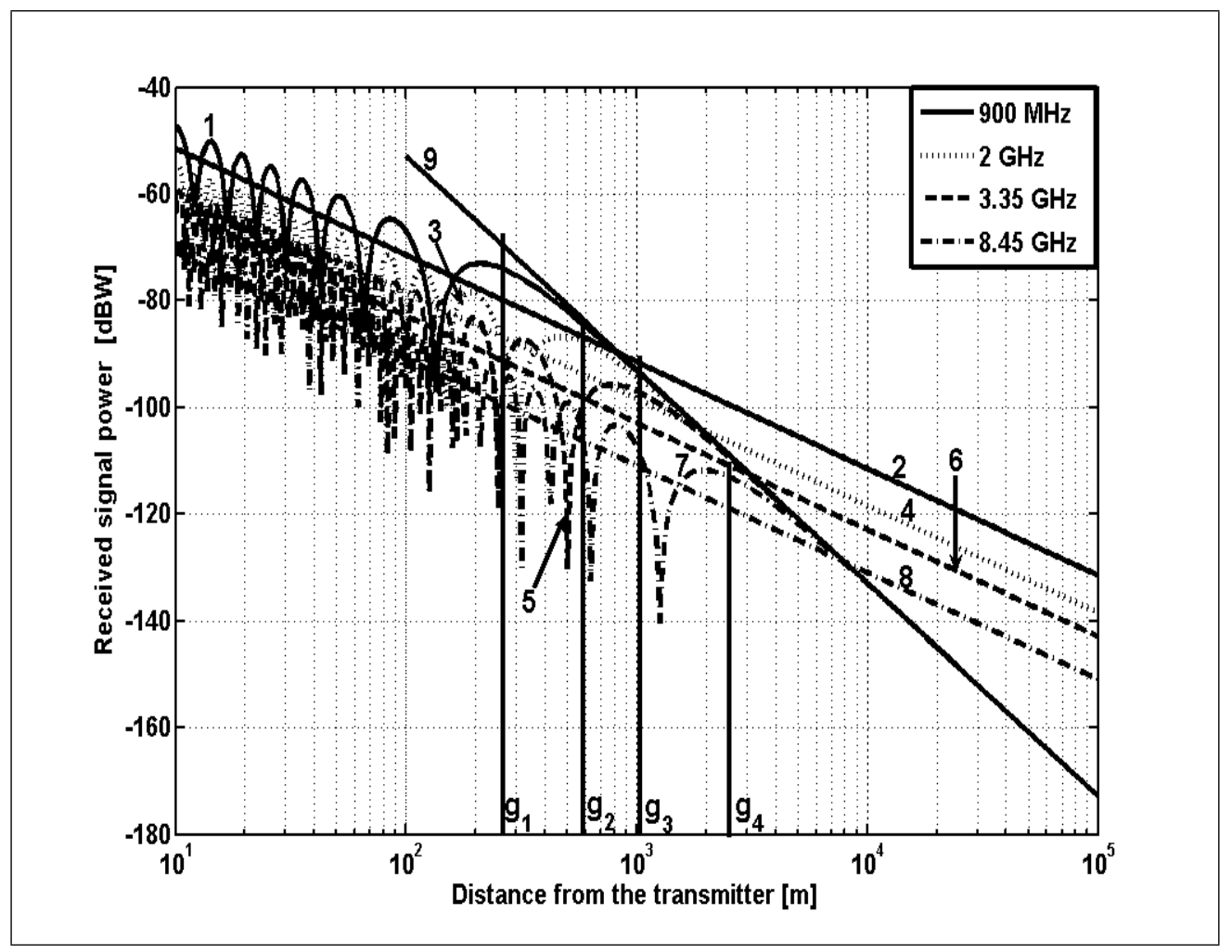

Figure 5.5: Two-slope path loss model received signal power $P_{r}$ versus distance $R$. Showing breakpoint distances $g_{1}, g_{2}, g_{3}$ and $g_{4}$ at carrier frequencies, $f_{c}=0.9,2,3.35$ and 8.45 GHz. (Transmitted power $P_{t}=1 \mathrm{~W}, \mathrm{MS}$ and BS antenna gain $G_{r}=G_{t}=1$, basic path loss exponent $\alpha=2$, extra path loss exponent $\rho=4$, MS and BS antenna heights: $h_{m}=1.5 \mathrm{~m}$ and $h_{b}=15 \mathrm{~m}$ )

1. Two Ray path loss at $0.9 \mathrm{GHz}$ [68]

2. Free Space Path Loss at $0.9 \mathrm{GHz}[86]$

3. Two Ray path loss at $2 \mathrm{GHz}[68]$

4. Free Space Path Loss Model at $2 \mathrm{GHz}$ [86]

5. Two Ray path loss at $3.35 \mathrm{GHz}[68]$

6. Free Space Path Loss Model at $3.35 \mathrm{GHz}$ [86]

7. Two Ray path loss at $8.45 \mathrm{GHz}$ [68]

8. Free Space Path Loss Model at $8.45 \mathrm{GHz}$ [86]

9. Power falls off $\propto 1 /(\text { distance })^{4}$ 
to show that as the break point distance increases other tiers of co-channel interfering cell become active. This other tiers co-channel interfering cell leads to a decrease in the information capacity of the cellular wireless system.

\subsubsection{Relationship between Breakpoint Distance, Cell Size Radius and Number of Co-channel Tiers}

In this section, the relationship between breakpoint distance, cell size radius and the number of active co-channel interfering tiers is explained intuitively, whilst it will become clearer as we go through section 5.4, using mathematical analysis. To show this relationship, we plot a geometrical circular cell site layout. The plot shows the breakpoint distance $g$, for different carrier frequencies, $f_{c}=0.9,2,3.35$ and $8.45 \mathrm{GHz}$, and cell radius size $R$ $=100 \mathrm{~m}$. Figure 5.6, shows the plotted circular cell site layout.

From Figure 5.6, a cellular system with cluster size $N_{c}$, will have its first tier cochannel interfering cells on a circle of radius $D=\sqrt{3 \times N_{c}} R$. $R$, being the cell radius. For a cell size radius $R=100 \mathrm{~m}$, and cluster size, $N_{c}=7$, the reuse distance is given by $D=$

$\sqrt{3 \times 7} \times 100 \approx 458 \mathrm{~m}$. That is, first tier co-channel interfering cells will be on a circle of radius $D=458 \mathrm{~m}$, from the desired MS's base station $\mathrm{BS}_{0}$ (refer to Figure 5.6). A second tier co-channel interfering cell, reuse distance $D_{1}$, will be on a circle of radius twice that of the radius for the first tier co-channel cells. Hence, $D_{1}=2 \times 458 \mathrm{~m} \approx 916 \mathrm{~m}$. For a third tier co-channel interfering cell, it will be on a circle of radius $D_{2}$, which is thrice the radius $D$ of the first tier co-channel cells. Therefore $D_{2}=3 \times 458 \mathrm{~m} \approx 1374 \mathrm{~m}$. It follows, that the nth tier reuse distance $D_{n}$ is the product of the nth tier number $(\mathrm{n}=1,2,3,4, \ldots)$, and first tier reuse distance $D,\left(D_{n}=\mathrm{n} \times D\right)$. Figure 5.6, shows that for $f_{c}=900 \mathrm{MHz}$, the first tier co-channel interfering cells are after the breakpoint, $g_{1}$. When $f_{c}=2 \mathrm{GHz}$, the breakpoint $g_{2}$, is before the second tier co-channel interfering cells. In the case of $f_{c}$ $=3.35 \mathrm{GHz}$, the breakpoint $g_{3}$, is after the second tier co-channel interfering cells. For $f_{c}=8.45 \mathrm{GHz}$, the breakpoint $g_{4}$, is after the third tier co-channel interfering cells. From the explanation, we can conclude that as carrier frequency increases, and cell size radius reduces, the number of co-channel interfering tiers before the breakpoint increases. 


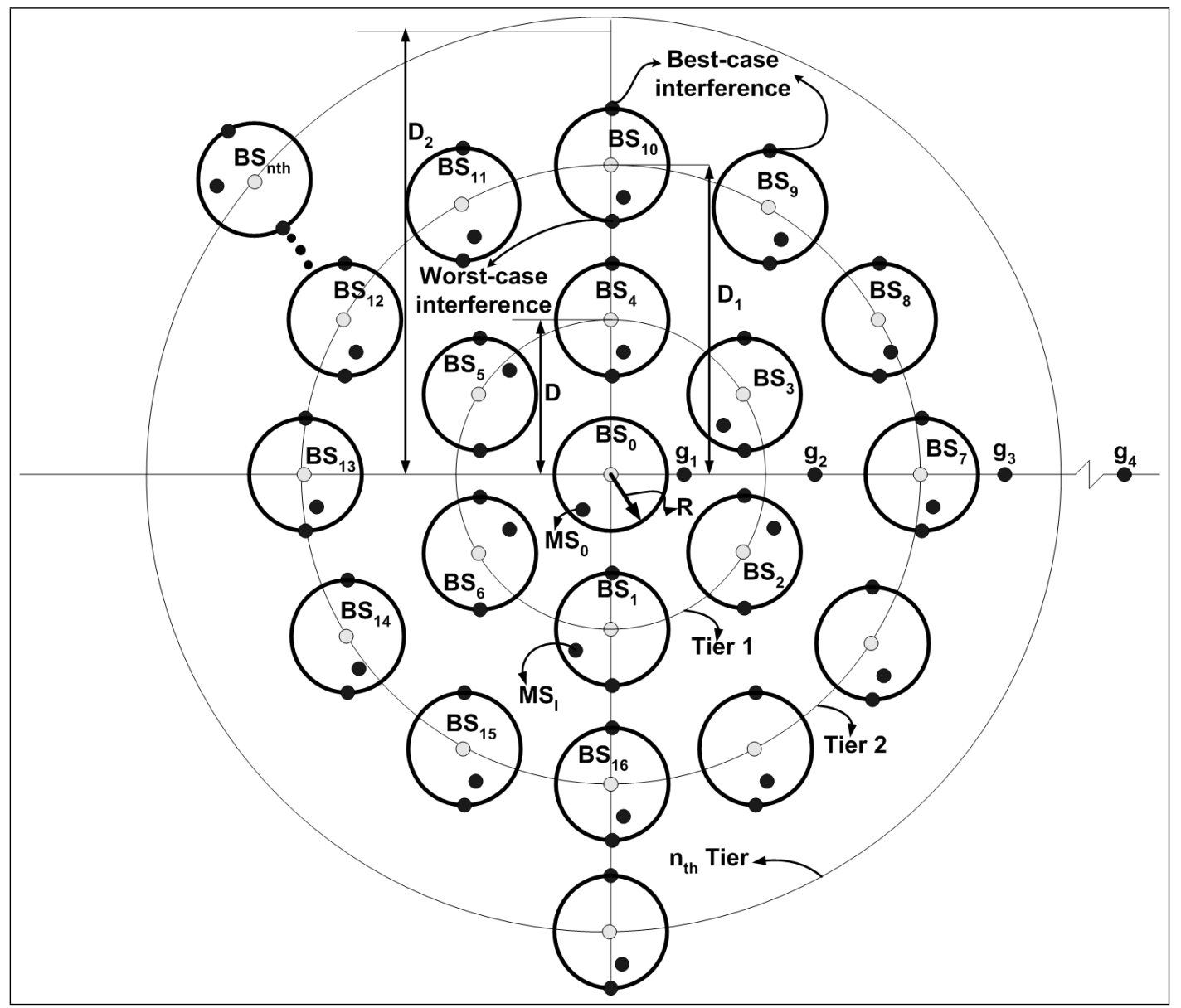

Figure 5.6: Uplink co-channel interference on a desired BS of a fully loaded nonsectorized cellular system, with 6, 12 and n co-channel interference cells in first, second and nth tier. (Cell radius $R=100 \mathrm{~m}$, cluster size $N_{c}=7$ and breakpoint distance $g_{1}=270$ $\mathrm{m}, g_{2}=600 \mathrm{~m}, g_{3}=1005 \mathrm{~m}$ and $g_{4}=2535 \mathrm{~m}$, at microwave carrier frequencies, $f_{c}=0.9$, 2, 3.35 and $8.45 \mathrm{GHz}$ ), BS antenna height, $h_{b}=15 \mathrm{~m}$ and MS antenna height, $h_{m}=1.5 \mathrm{~m}$. 


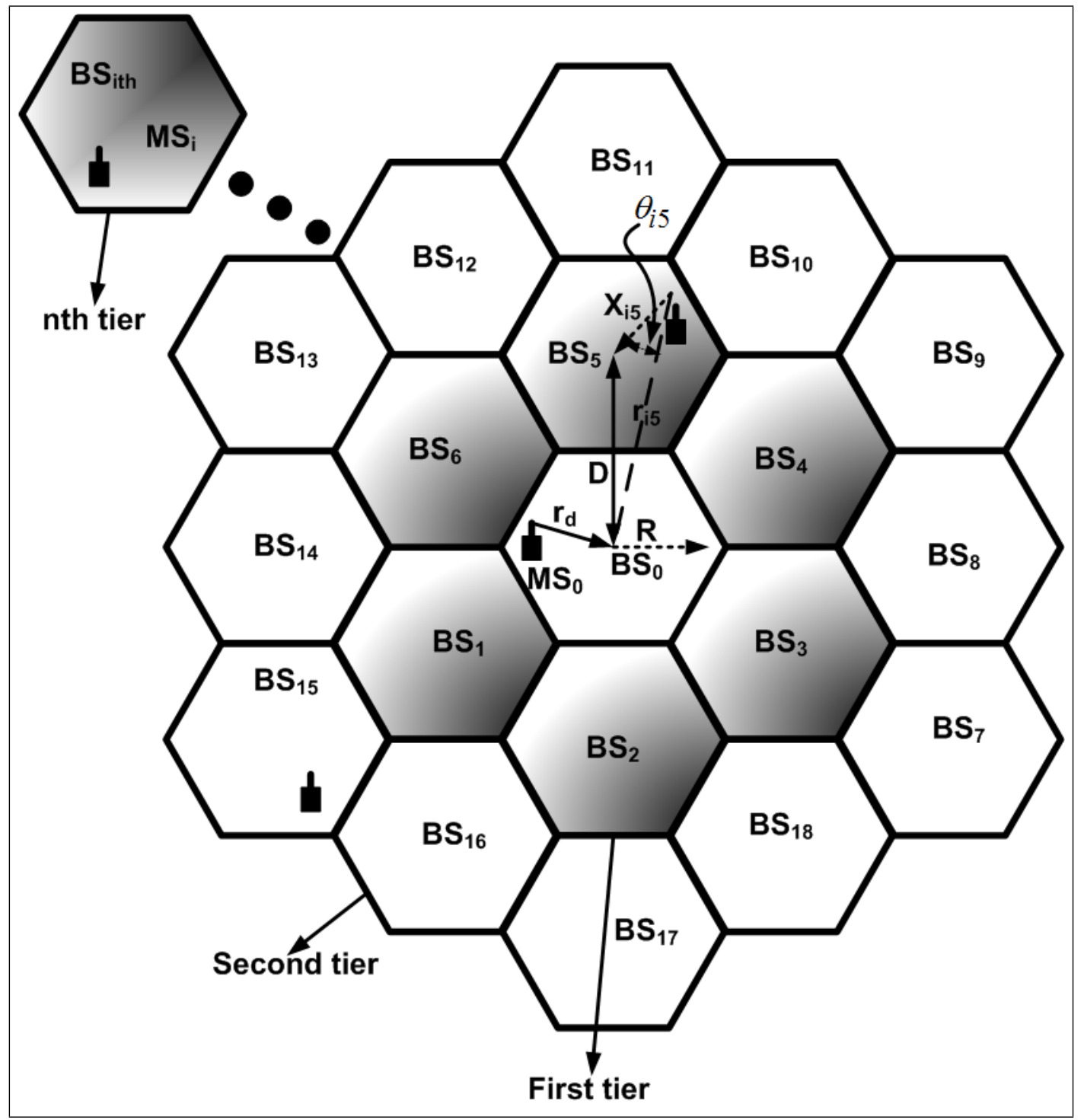

Figure 5.7: Multiple tier uplink co-channel interference on a desired BS of a fully loaded non-sectorized cellular system site layout, showing $\mathrm{n}$ tiers of co-channel interfering cells.

1. $\mathrm{BS}_{0}$ : the reference cell

2. $\mathrm{MS}_{0}$ : the desired MS

3. $\mathrm{R}$ : the cell radius

4. $\mathrm{BS}_{n t h}$ : the nth tier BS 
As stated in previous sections, before the breakpoint, power falls off in inverse proportion to the second power of distance. After the breakpoint power falls off in inverse proportion to the fourth power of distance. Therefore insofar as much as co-channel interfering cells are before the breakpoint, they tend to become active.

\subsubsection{Mobile and Interfering Users Distribution in Cells}

As stated earlier in section 4.2, the hexagonal cell site layout shape is approximated by a circle of radius $R$. This approximation is for mathematical convenience, and accuracy during our analysis. Therefore, the probability distribution function (PDF) of a MS location relative to a BS is described by equation (4.1) in subsection 4.2.1.

\subsection{Multiple Tier Interference Modelling}

The system for our interference modelling is a two-dimensional hexagonal non-sectorized smaller cell size network. The system is considered to have multiple tiers of co-channel interfering cells. Base stations are assumed to be uniformly distributed. The system model is depicted in Figure 5.7. For the system model the hexagonal cells have been approximated by circular cells with equal area, for mathematical simplicity and accuracy as in $[89,90]$. All cells are assumed to have omnidirectional antennas. From Figure 5.7, cell $\mathrm{BS}_{0}$, is referred to as the reference cell, with n-tiers of co-channel interfering cells surrounding it. Now a cell $j$ is served by $\mathrm{BS}_{j}$, which is located at the cell's center, with $1 \leq \mathrm{j} \leq 6$, for first tier co-channel interferes, $7 \leq \mathrm{j} \leq 18$, for second tier co-channel interferes, and $[((\mathrm{n}$ - 1) $\times 6)+1] \leq \mathrm{j} \leq[((\mathrm{n}-1) \times 6)+6 \mathrm{n}]$, for the $n$th tier co-channel interferes. Let $D$ (refer to Figure. 5.7) stand for the distance between $\mathrm{BS}_{0}$ and a first tier BS. A second tier BS is then at a distance of $2 D$ from $\mathrm{BS}_{0}$. An nth tier $\mathrm{BS}$ will be located at distance $\mathrm{n} D$. We consider each cell site to be having a single user for the modelling.

\subsubsection{General Interference Model}

We consider a cellular system with one user per cell and multiple cells. For the uplink, each BS receives co-channel interference from $M$ users in neighboring BSs'. The number 
of $M$ interferers depends on the cell site geometric layout and active co-channel interfering tiers.

\subsubsection{Proposed Model - Multiple Tier Interference}

For the proposed multiple tier co-channel interference model, we consider an uplink cellular wireless network consisting of MSs' and BSs' as shown in Figure. 5.7. The system under consideration has a single - user (desired MS), of interest affected by co-channel interference from multiple users (interfering MSs'), from multiple tier co-channel cells. The system is considered to be interference-limited (meaning that the receiver noise power is generally much less than the interference power). Thermal noise power is negligible relative to the co-channel interference power. Inter-channel interferences are also considered to be negligible. Mobile stations are assumed to transmit the same power and radio channels fluctuate according to path loss. The inter - cell interference is dependent on the frequency reuse factor. Note that we do not use power control in this model, because it is essential in direct sequence CDMA networks. Considering the desired MS to be located in the central cell $\left(\mathrm{BS}_{0}\right)$ of a cellular wireless network and the interfering MSs' to be located in co-channel interfering cells. The desired user carrier-to-interference ratio, $C I R$, $\gamma$, is defined as the ratio of the average received signal power level, $P_{d}[\mathrm{~W}]$ from a MS at a distance $r[\mathrm{~m}]$, from the desired BS to the sum $P_{I}[\mathrm{~W}]$, of interfering power at distance $r_{i 1}, r_{i 2}, \cdots, r_{i n}$ from the interfering BSs'. We therefore write the desired user $C I R, \gamma$, for the multiple tier co-channel interference as follows

$$
\gamma=\frac{P_{d}}{P_{I}}=\frac{P_{d}(r)}{\sum_{i 1=1}^{N_{I 1}} P_{i 1}\left(r_{i 1}\right)+\sum_{i 2=1}^{N_{I 2}} P_{i 2}\left(r_{i 2}\right)+\cdots+\sum_{i n=1}^{N_{I n}} P_{i n}\left(r_{i n}\right)}
$$

where $P_{i 1}, P_{i 2}$ and $P_{i n}[\mathrm{~W}]$ are the average received power from the ith interfering BSs'. $N_{I 1}, N_{I 2} \cdots, N_{I n}$ are the maximum number of co-channel interferers in the respective tiers.

The interference model applies to bandwidth limited systems, such as TDMA, and it is based on a fully loaded non-sectorized cellular wireless communication system. The analysis from previous chapters has shown that $\gamma$, is a function of received signal power from the desired and interfering users. As we have stated earlier the desired MS's and 
interfering MSs' are randomly located, $\gamma$ is also a random variable (RV). In a smaller cell size radius wireless environment, where frequencies are reused over a urban area with different cluster size $N_{c}$, for a hexagonal tessellation with $N_{c}=7$, the total number of cochannel interfering cells in the first tier equals to six. The number of co-channel interfering cells in a known tier is given by $6 \times n$ [45], where $n$ is the $n$th tier. For a cellular wireless communication system with $N_{c}=7$, the desired user $C I R, \gamma$ for the multiple tier co-channel interference is given by:

$$
\gamma_{d}=\frac{P_{d}}{P_{I}}=\frac{P_{d}(r)}{\sum_{i 1=1}^{6} P_{i 1}\left(r_{i 1}\right)+\sum_{i 2=1}^{12} P_{i 2}\left(r_{i 2}\right)+\cdots+\sum_{i n=1}^{6 \times N_{n}} P_{i n}\left(r_{i n}\right)}
$$

where $N_{n}$ is the maximum number of co-channel interfering tiers. It is assumed that all MS transmit the same power. A distance dependent path loss is assumed and for simplicity and clarity $P$ is dropped from equation (5.3), without loss of generality. Using equation (5.2), (5.4), and substituting the reuse distance $D$, with the product of the normalized reuse distance $R_{u}$, and cell size radius $R$ (that is, $D=R_{u} R$ ), we obtain the desired MS's $C I R$ as follows

$$
\begin{aligned}
& \gamma_{d}=\frac{P_{d}}{P_{I}}=\frac{r^{-\alpha}(1+r / g)^{-\rho}}{\sum_{i 1=1}^{6} r_{i 1}^{-\alpha}\left(1+r_{i 1} / g\right)^{-\rho}+\sum_{i 2=1}^{12} r_{i 2}^{-\alpha}\left(1+r_{i 2} / g\right)^{-\rho}+\cdots+\sum_{i n=1}^{6 \times N_{n}} r_{i n}^{-\alpha}\left(1+r_{i n} / g\right)^{-\rho}} \\
& =\frac{r^{-\alpha}(1+r / g)^{-\rho}}{\sum_{i=1}^{6} D^{-\alpha}(1+D / g)^{-\rho}+\sum_{i=1}^{12}(2 D)^{-\alpha}(1+(2 D) / g)^{-\rho}+\cdots+\sum_{i=1}^{6 \times N_{n}}\left(N_{n} D\right)^{-\alpha}\left(1+\left(N_{n} D\right) / g\right)^{-\rho}} \\
& =\frac{\frac{1}{r}^{\alpha}\left(1+\frac{r}{g}\right)^{-\rho}}{\sum_{i=1}^{6} \frac{1}{R R_{u}^{\alpha}}\left(1+\frac{\left(R R_{u}\right)}{g}\right)^{-\rho}+\sum_{i=1}^{12} \frac{1}{\left(2 R R_{u}\right)^{\alpha}}\left(1+\frac{\left(2 R R_{u}\right)}{g}\right)^{-\rho}+\cdots+\sum_{i=1}^{6 \times N_{n}} \frac{1}{\left(N_{n} R R_{u}\right)^{\alpha}}\left(1+\frac{\left(N_{n} R R_{u}\right)}{g}\right)^{-\rho}}
\end{aligned}
$$

Note that $\left(N_{n} D\right)$, is the reuse distance between the $N_{n}$ tier co-channel cell and the desired BS. Using the summation properties, and representing the total tiers of co-channel interferers by $N_{n}$ and simplifying (5.5), we can write the desired MS's CIR as follows

$$
\gamma_{d}\left(N_{n}, r,\right)=\frac{r^{-\alpha}(1+r / g)^{-\rho}}{\sum_{i=1}^{\infty} \sum_{n=1}^{6 N_{n}}\left(n R_{u} R_{i n}\right)^{-\alpha}\left(1+\left(n R_{u} R_{i n}\right) / g\right)^{-\rho}} .
$$

\subsection{Information Capacity - Multiple Tier Interference}

We still quantify the information capacity, for our impact analysis by the Area Spectral Efficiency $(A S E)$, since the ultimate capacity of a land mobile radio system is directly 


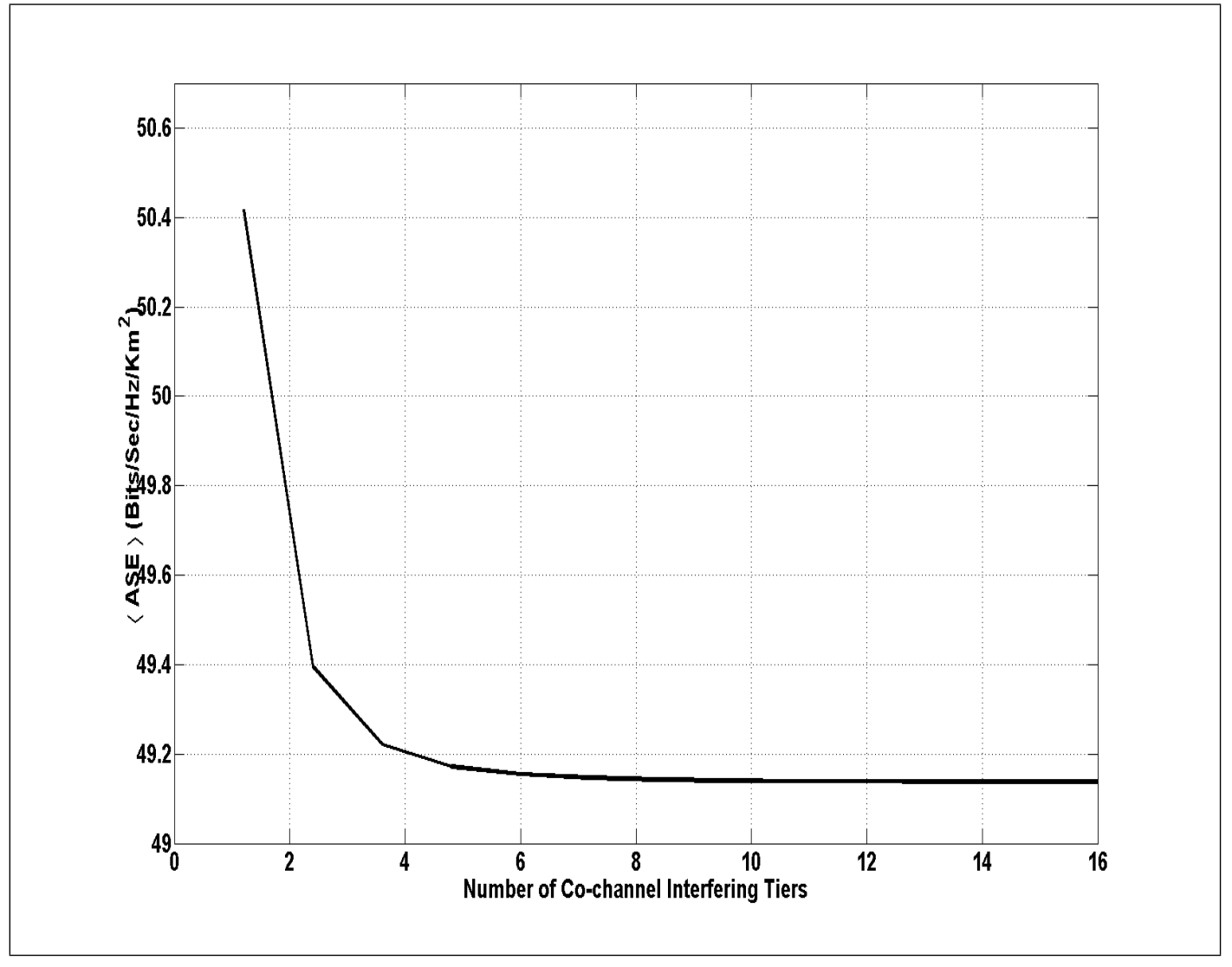

Figure 5.8: Average area spectrum efficiency versus number of co-channel interfering tiers. Path loss exponent, $\alpha=2$, extra path loss exponent $\rho=4$, carrier frequency $f_{c}=900$ MHz, cell size radius $R=100 \mathrm{~m}, \mathrm{MS}$ and BS antenna heights : $h_{m}=1.5 \mathrm{~m}$ and $h_{b}=15 \mathrm{~m}$.

related to its spectral efficiency [29], and also for the consistence of this thesis. As stated earlier in Chapter 4.3, the $A S E$ used for the analysis is in relationship to a fully loaded cellular wireless system. However, this time around the interference are assumed to be coming from multiple tiers. Using the same notation in Subsection 4.3.1, such that $N_{s}$ represent the total number of active serviced channels per cell, $C_{k}[\mathrm{bits} / \mathrm{sec}]$ the maximum data rate of the kth user. $W[\mathrm{~Hz}]$ the total bandwidth allocated to each cell. $D$ the frequency reuse distance. The $A S E$ is expressed by equation (4.2) as in Chapter 4.3.

For this work both multiple tier interference, and signal the power of the kth user varies with mobile locations and propagation conditions. Therefore, the carrier-to-interference ratio $C I R, \gamma$ will also vary with time. Hence the information capacity, $C_{k}$ of the kth user equals the average channel capacity of that kth user and is given by equation (4.5), refer to Chapter 4.3. 


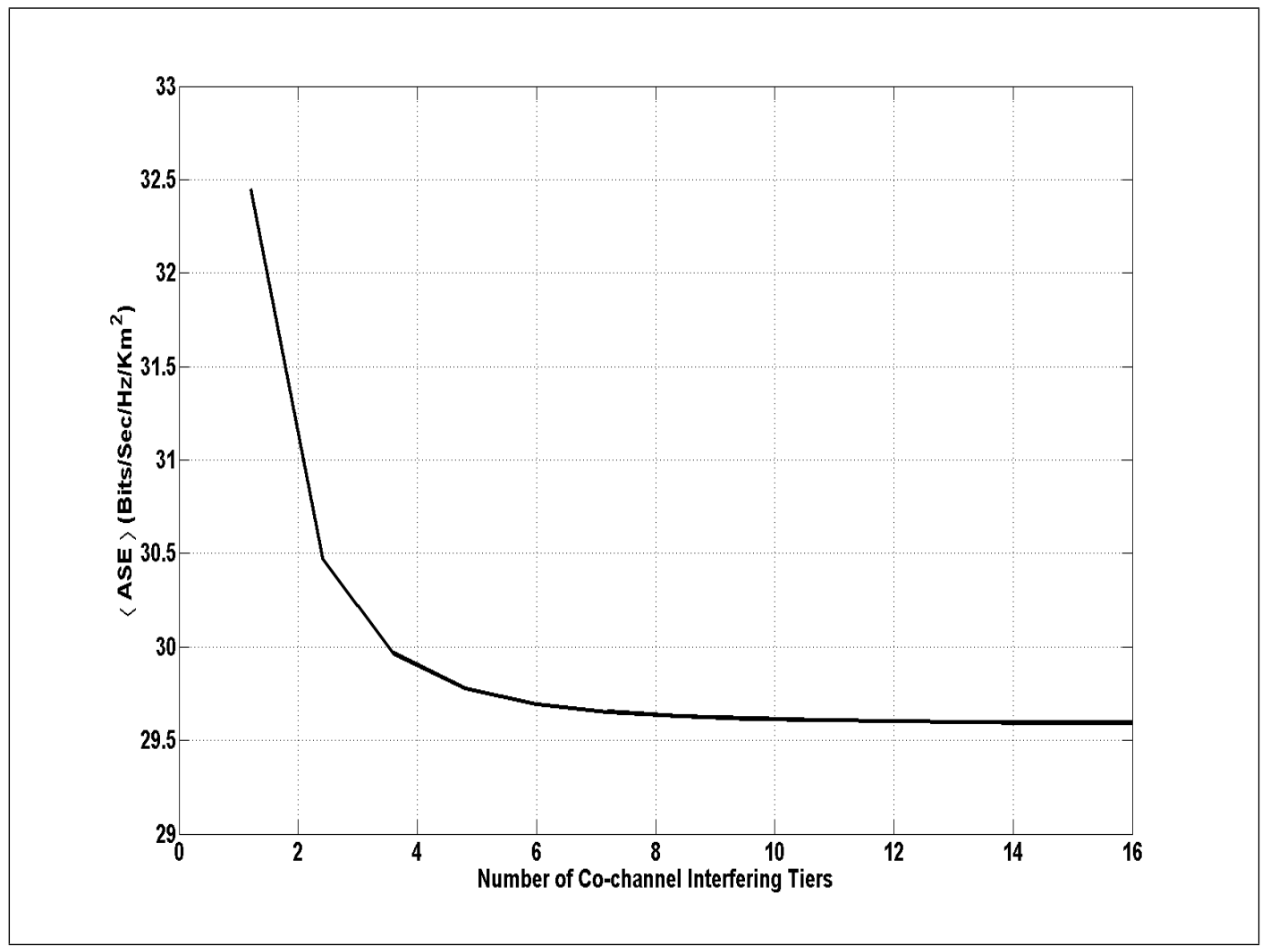

Figure 5.9: Average area spectrum efficiency versus number of co-channel interfering tiers. Path loss exponent, $\alpha=2$, extra path loss exponent $\rho=4$, carrier frequency $f_{c}=3.35$ GHz, cell size radius $R=100 \mathrm{~m}, \mathrm{MS}$ and BS antenna heights : $h_{m}=1.5 \mathrm{~m}$ and $h_{b}=15 \mathrm{~m}$.

\subsubsection{Tiers of Co-channel Interfering Cell for Consideration in Smaller Cell Size Radius System}

In this section, we find the maximum number of co-channel tiers $N_{n}$ to be considered in our analysis and simulation. To justify the choice for $N_{n}$ we consider an arrangement of hexagonal coverage area as described in section 5.3. Using equations (5.6) and (4.5), a graph of area spectral efficiency versus number of co-channel interfering tiers, for cell radius $R=100 \mathrm{~m}$ and carrier frequencies $f_{c}=0.9,3.35$ and $15.75 \mathrm{GHz}$ is plotted. Noting that for hexagonal coverage, there are 6 co-channel interfering cells in the first tier and $6 n$ co-channel interfering cells in the nth tier.

The curve in Figure 5.8 is for carrier frequency $f_{c}=900 \mathrm{MHz}$, the curve shows that the area spectral efficiency $A S E$ tends to remain constant after the third tier. Figure 5.9 shows the curve for carrier frequency $f_{c}=3.35 \mathrm{GHz}$. The curve shows that the area 


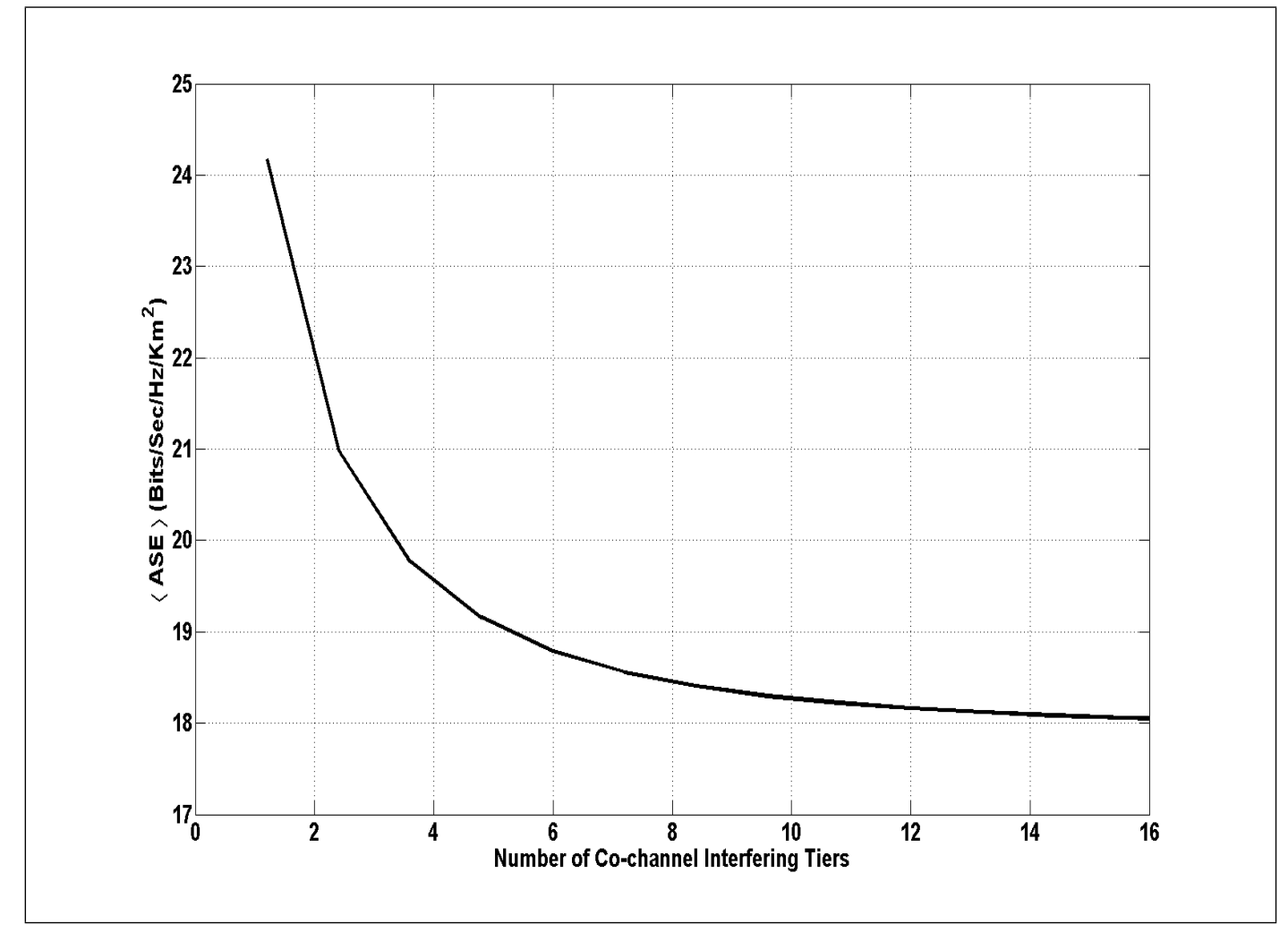

Figure 5.10: Average area spectrum efficiency versus number of co-channel interfering tiers. Path loss exponent, $\alpha=2$, extra path loss exponent $\rho=4$, carrier frequency $f_{c}=$ $15.75 \mathrm{GHz}$, cell size radius $R=100 \mathrm{~m}$, MS and BS antenna heights : $h_{m}=1.5 \mathrm{~m}$ and $h_{b}=$ $15 \mathrm{~m}$.

spectral efficiency $A S E$ tends to be constant after the fifth tier. Figure 5.10 depicts the curve for carrier frequency $f_{c}=15.75 \mathrm{GHz}$. The curve shows that the area spectral efficiency $A S E$ tends to be the same after the tenth tier. From Figures $5.8-5.10$, it is concluded that for the chosen carrier frequencies used in this thesis, the maximin number of co-channel interfering tier $N_{n}$ used for analysis and simulation should be ten tiers.

\subsection{Propagation/System Parameter Impact Analysis}

This analysis applies to bandwidth limited systems such as TDMA, and it is based on a fully loaded non-sectorized cellular wireless communication system. Our analysis so far has shown that $\gamma$ is a function of received signal power from the desired and interfering users. Since we assume transmission rate to be continuously adapted relative to the $C I R$ in such a manner that the $B E R$ goes to zero asymptotically. If we assume that all user 
are assigned the same bandwidth, $\left\langle C_{k}\right\rangle=(\langle C\rangle)$, becomes the same for all users, therefore $\left\langle A_{e}\right\rangle$ can be written as

$$
\left\langle A_{e}\right\rangle=\frac{4 N_{s}\langle C\rangle}{\pi B D^{2}}=\frac{4 N_{s}\langle C\rangle}{\pi W R_{u}^{2} R^{2}},
$$

In a TDMA system, the total bandwidth is allocated to only one active mobile user per time slot. Keeping in mind that for the multiple tier interference impact analysis, we still consider a TDMA system for consistence of the thesis. The equation for the information capacity, which is the $A S E$ remains the same as that of equation (4.5).

Since the average $C I R$ of the desired MS, $\gamma$ is a function of both the number of co-channel interferers, and the desired MS's position, $r$, the desired MS's capacity remains the same as equation (4.7). However, we replace $N_{F I}$ and $N_{S I}$, the first and second tier co-channel interfering cells by the total number of the multiple tier interfering cells. Integrating the equation, over the desired MS's position PDF equation (4.1), yields the average information capacity $A S E$ for the multiple tier co-channel interference configuration as presented by equation (4.8).

\subsection{Numerical Simulation Setup for Propagation/System Parameter Impact Analysis}

Computer simulation by Monte Carlo method is provided to compare the information capacity performance, between the conventional and our proposed multiple tier co-channel interference model. The comparison is for different propagation loss/system parameters at higher microwave carrier frequency and smaller cell size radius. Without loss of generality only ten tiers of co-channel interfering cells were considered for interference generation for simulation. Ten tiers were considered, because by successive numerical runs with an increased number of tiers, we found that the addition of further tiers beyond ten had no effect on the accuracy of the results. Note also that in subsection 5.4.1 we justify the use of ten tiers.

For the multiple tier interference simulation, the simulation environment is similar to that of Figure 4.2. The simulation model geometry is as shown in Figure 5.11. Figure 5.12, shows a simplified flow chart for the simulation setup. First the desired MS 


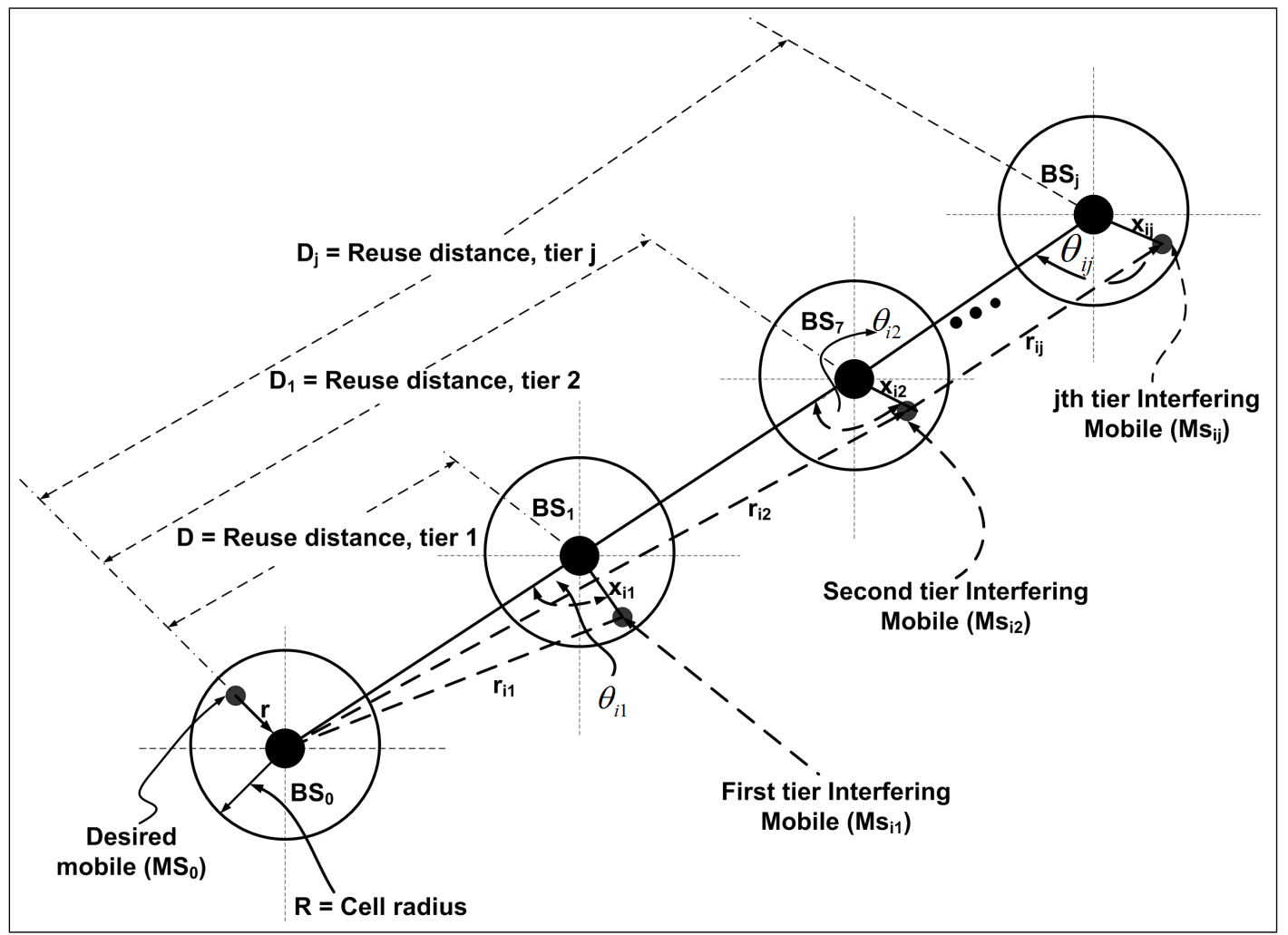

Figure 5.11: Geometry of desired user and interfering users in different co-channel tiers.

1. $\mathrm{BS}_{0}$ : the reference cell

2. $\mathrm{BS}_{1}$ : first tier co-channel interfering cell

3. $\mathrm{BS}_{7}$ : second tier co-channel interfering cell

4. $\mathrm{BS}_{j}$ : jth tier co-channel interfering cell

5. $\mathrm{D}$ : distance of separation between $\mathrm{BS}_{0}$ and $\mathrm{BS}_{1}$

6. $\mathrm{D}_{1}$ : distance of separation between $\mathrm{BS}_{0}$ and $\mathrm{BS}_{7}$

7. $\mathrm{D}_{j}$ : distance of separation between $\mathrm{BS}_{0}$ and $\mathrm{BS}_{j}$

8. $\mathrm{r}$ : distance of separation between $\mathrm{MS}_{0}$ and $\mathrm{BS}_{0}$

9. $\mathrm{r}_{i 1}$ : distance of separation between $\mathrm{BS}_{0}$ and $\mathrm{X}_{i 1}$

10. $\mathrm{r}_{i 2}$ : distance of separation between $\mathrm{BS}_{0}$ and $\mathrm{X}_{i 2}$

11. $r_{i j}$ : distance of separation between $B_{0}$ and $X_{i j}$

12. $\theta_{i 1}$ : direction of $\mathrm{MS}_{i 1}$ travel referenced to a vector from $\mathrm{BS}_{0}$ to $\mathrm{BS}_{1}$

13. $\theta_{i 2}$ : direction of $\mathrm{MS}_{i 2}$ travel referenced to a vector from $\mathrm{BS}_{0}$ to $\mathrm{BS}_{7}$

14. $\theta_{i j}$ : direction of $\mathrm{MS}_{i j}$ travel referenced to a vector from $\mathrm{BS}_{0}$ to $\mathrm{BS}_{7}$ 


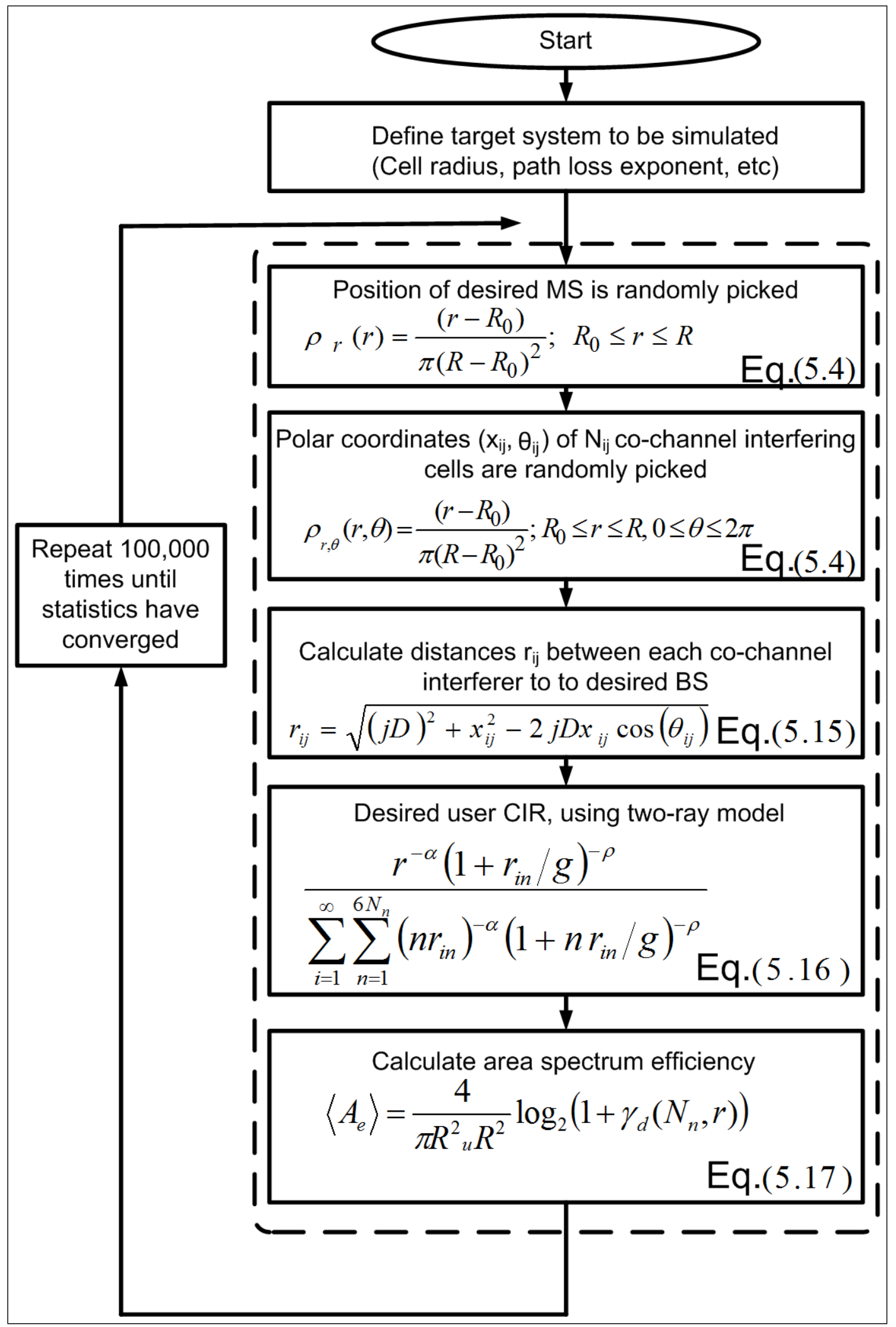

Figure 5.12: Flow chart for multiple tier interference information capacity simulations. 
position is located using the uniform distribution given by equation (4.1). The polar coordinates (xij,, $\mathrm{ij})$, of the jth interfering MS position of the $N \mathrm{ij}$ co-channel interfering mobiles are also located according to the uniform distribution. The limits of the distribution are in the range of $[0,2 \pi]$, with respect to current position of MS. To ensure consistence in this thesis the same simulation parameters used in Chapter 4.4.1, is used for the simulation in this chapter. From the simulation model geometry in Figure 5.11, the distance $r_{i j}$ is given as

$$
r_{i j}=\sqrt{(j D)^{2}+x_{i j}^{2}-2 j D x_{i j} \cos \left(\theta_{i j}\right)} .
$$

Using the two-slope path loss model the desired user CIR for the simulation is given by

$$
\gamma_{d}\left(N_{n}, r\right)=\frac{r^{-\alpha}(1+r / g)^{-\rho}}{\sum_{i=1}^{\infty} \sum_{n=1}^{6 N_{n}}\left(n r_{i n}\right)^{-\alpha}\left(1+n r_{i n} / g\right)^{-\rho}} .
$$

The information capacity given by the average $A S E, A_{e}$ for the simulation is given by

$$
\left\langle A_{e}\right\rangle=\frac{4}{\pi R_{u}^{2} R^{2}} \log _{2}\left(1+\gamma_{d}\left(N_{n}, r\right)\right) .
$$

We then estimated $\left\langle A_{e}\right\rangle$, by taking the average of all the observations of $A_{e}$, in equation (5.10), after repeating the simulation procedure 100000 times. The simulation procedure is repeated 100000 times so that the sample mean will converge to the true expected value.

\section{$5.7 \quad$ Numerical Results}

In this section, we present the results from the Monte-Carlo simulations, for the information capacity performance comparison between the conventional interference model and the proposed multiple tier interference model presented in Chapter 5.3. The results show that, (i) at higher microwave carrier frequencies greater than $2 \mathrm{GHz}$ and smaller cell size radius multiple tiers of co-channel interference become active and (ii) propagation loss and system parameters still affect the information capacity performance of a cellular wireless communication system when multiple tiers of co-channel interference are active.

Figures 5.13 - 5.17, illustrate the effect of reuse pattern and cell radius on information capacity performance of emerging cellular wireless communication networks. The 
Numerical Results

figures confirm that the average $A S E$ is an increasing function of reuse factor. The curves show that the decrease in information capacity between the two interferers model at carrier frequency of $900 \mathrm{MHz}$ was less, compared to higher carrier frequencies $f_{c}=2,3.35,8.45$ and $15.75 \mathrm{GHz}$.

The curves in Figures 5.18 - 5.22, shows the comparsion in information capacity performance between the two and multiple tier interference model. The curves show that the decrease in information capacity between the two interferers model, (two and Multiple tier) at carrier frequency of $900 \mathrm{MHz}$ was less, compared to higher carrier frequencies $f_{c}=$ 2, 3.35, 8.45 and $15.75 \mathrm{GHz}$. The curves also show that the decrease was less as compared to the case of the single tier and multiple tier as expected, because second tier co-channel interference are less as compared to that from that first tier.

The figures shows that multiple tiers of co-channel interfering cells caused the $A S E$ to decrease more at higher microwave carrier frequencies The decrease in $A S E$ also becomes larger as the cell size radius reduces. This illustrates the fact that at higher carrier frequencies and smaller cell size radius multiple tiers of co-channel interfering cells cause interference to be severe. The curves also illustrate the fact that the information capacity of a cellular wireless network depends on the cell size radius and the carrier frequency. 


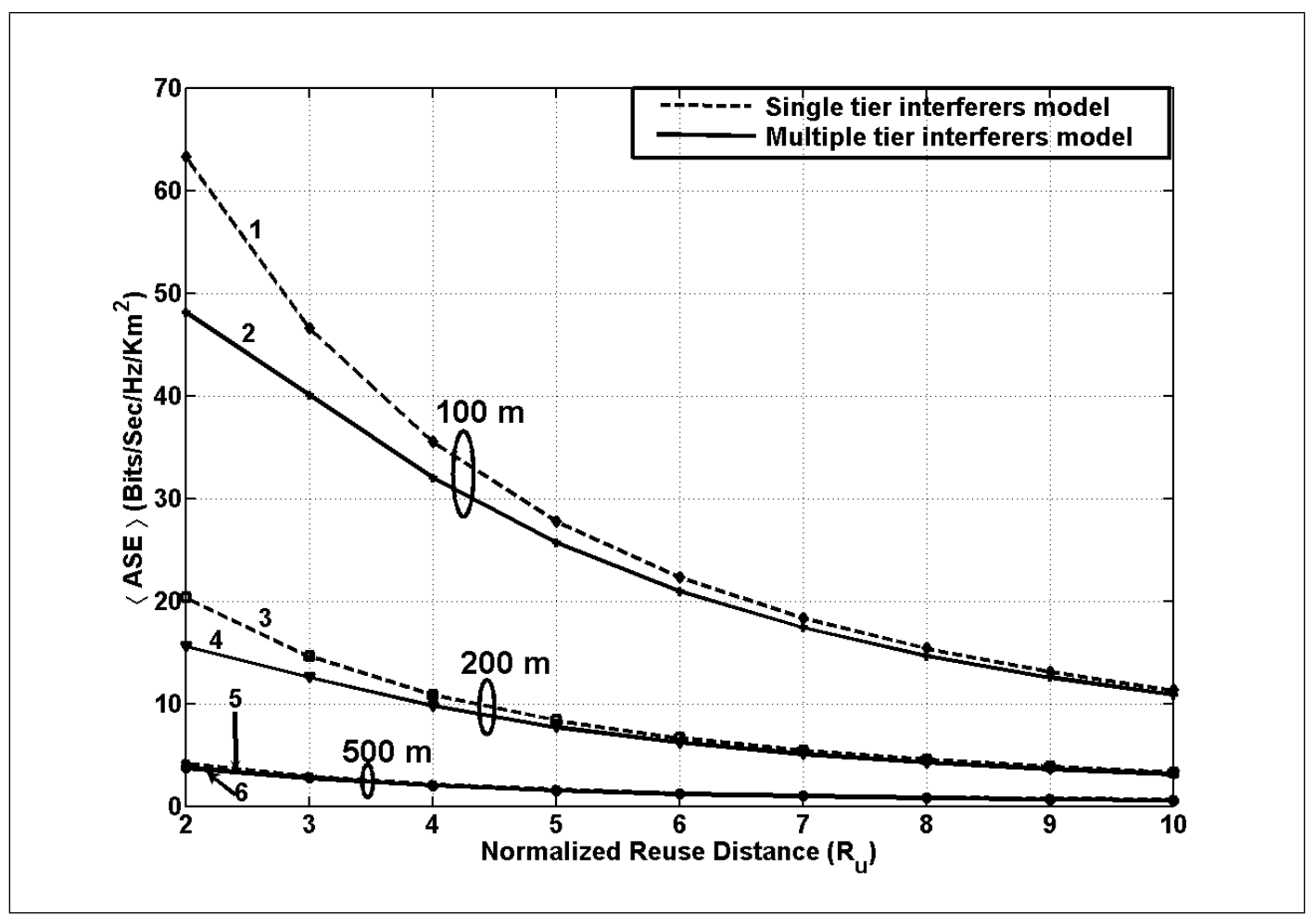

Figure 5.13: Comparison of the average area spectral efficiency, ASE for single and multiple tier interferers model for different normalized reuse distances $R_{u}$ and cell radius $R$. (Fully loaded non-sectorized cellular system; carrier frequency $f_{c}=900 \mathrm{MHz}$; path loss exponent $\alpha=2$ and extra path loss exponent $\rho=2$; BS antenna height $h_{b}=15 \mathrm{~m}$ and MS antenna height $h_{m}=1.5 \mathrm{~m}$; number of co-channel interfering cells; single tier $=6$ and multiple tier $=330$.

1. $A S E$ for conventional single tier co-channel interferers model, $R=100 \mathrm{~m}$ [77]

2. $A S E$ for proposed multiple tier co-channel interferers model, $R=100 \mathrm{~m}$

3. $A S E$ for conventional single tier co-channel interferers model, $R=200 \mathrm{~m}$ [77]

4. $A S E$ for proposed multiple tier co-channel interferers model, $R=200 \mathrm{~m}$

5. $A S E$ for conventional single tier co-channel interferers model, $R=500 \mathrm{~m}$ [77]

6. $A S E$ for proposed multiple tier co-channel interferers model, $R=500 \mathrm{~m}$ 


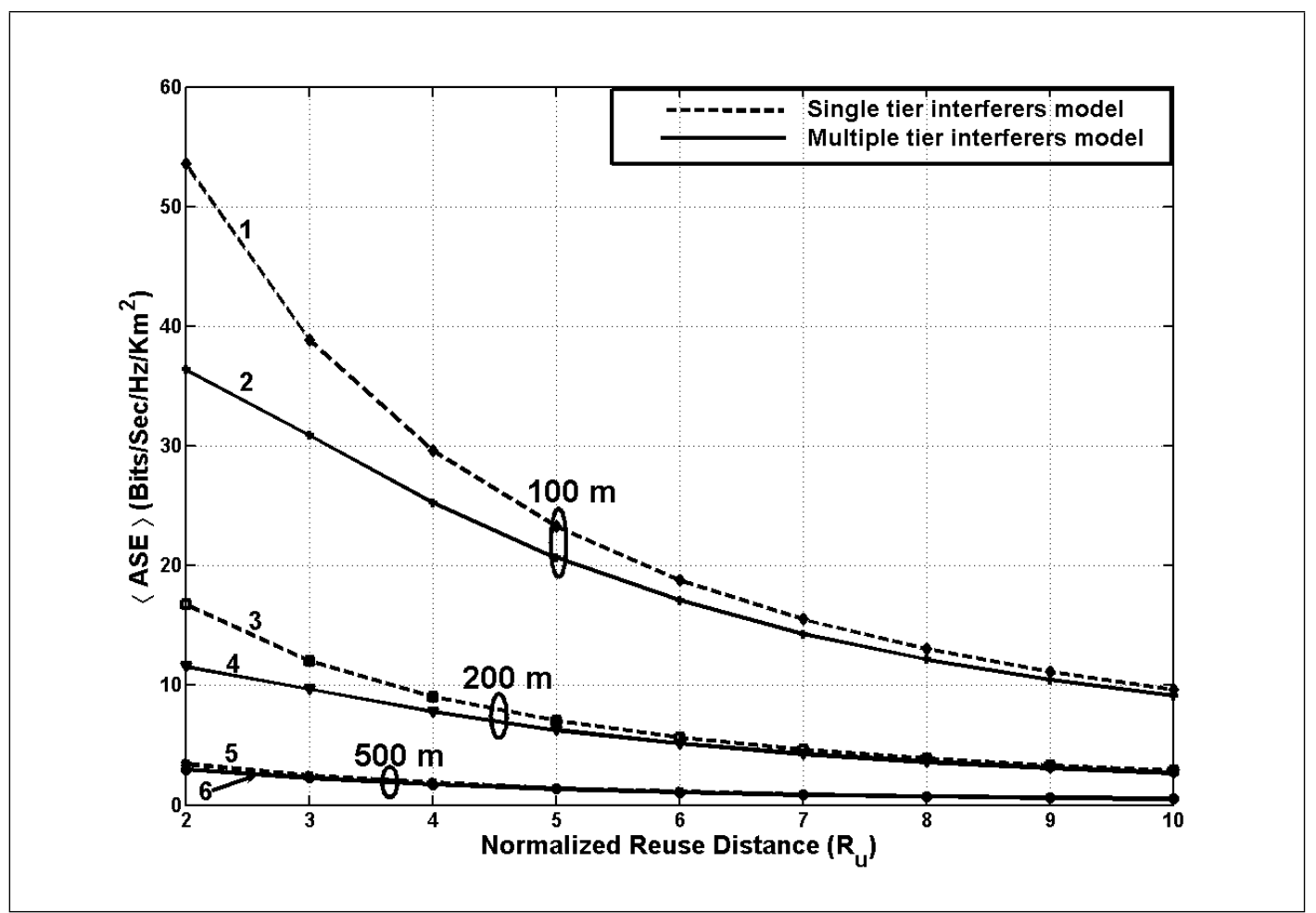

Figure 5.14: Comparison of the average area spectral efficiency, ASE for single and multiple tier interferers model for different normalized reuse distances $R_{u}$ and cell radius $R$. (Fully loaded non-sectorized cellular system; carrier frequency $f_{c}=2 \mathrm{GHz}$; path loss exponent $\alpha=2$ and extra path loss exponent $\rho=2$; BS antenna height $h_{b}=15 \mathrm{~m}$ and MS antenna height $h_{m}=1.5 \mathrm{~m}$; number of co-channel interfering cells; single tier $=6$ and multiple tier $=330$ )

1. ASE for conventional single tier co-channel interferers model, $R=100 \mathrm{~m}$ [77]

2. $A S E$ for proposed multiple tier co-channel interferers model, $R=100 \mathrm{~m}$

3. $A S E$ for conventional single tier co-channel interferers model, $R=200 \mathrm{~m}$ [77]

4. $A S E$ for proposed multiple tier co-channel interferers model, $R=200 \mathrm{~m}$

5. $A S E$ for conventional single tier co-channel interferers model, $R=500 \mathrm{~m} \mathrm{[77]}$

6. $A S E$ for proposed multiple tier co-channel interferers model, $R=500 \mathrm{~m}$ 


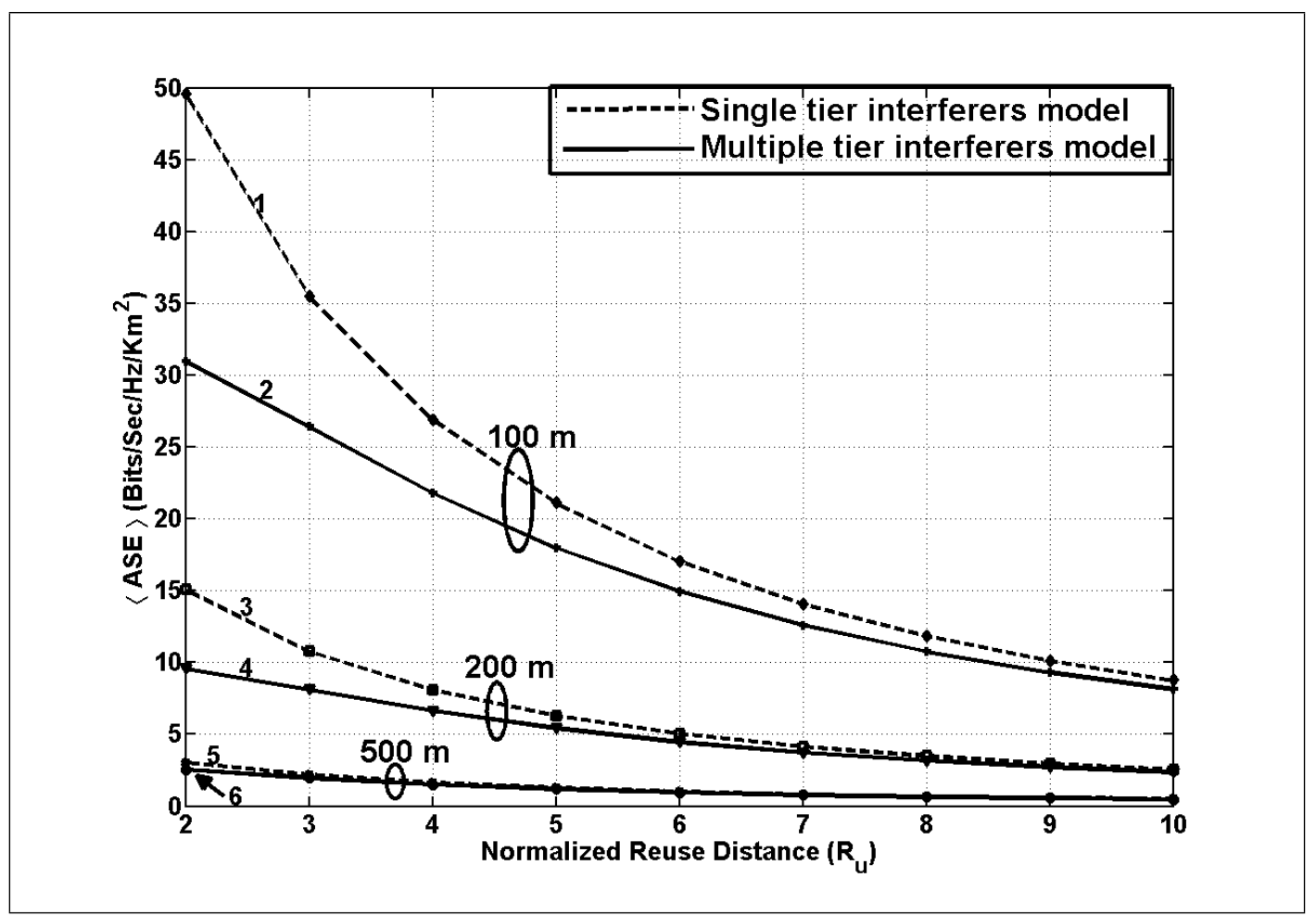

Figure 5.15: Comparison of the average area spectral efficiency, ASE for single and multiple tier interferers model for different normalized reuse distances $R_{u}$ and cell radius $R$. (Fully loaded non-sectorized cellular system; carrier frequency $f_{c}=3.35 \mathrm{GHz}$; path loss exponent $\alpha=2$ and extra path loss exponent $\rho=2$; BS antenna height $h_{b}=15 \mathrm{~m}$ and MS antenna height $h_{m}=1.5 \mathrm{~m}$; number of co-channel interfering cells; single tier $=6$ and multiple tier $=330$ )

1. ASE for conventional single tier co-channel interferers model, $R=100 \mathrm{~m}$ [77]

2. $A S E$ for proposed multiple tier co-channel interferers model, $R=100 \mathrm{~m}$

3. $A S E$ for conventional single tier co-channel interferers model, $R=200 \mathrm{~m}$ [77]

4. $A S E$ for proposed multiple tier co-channel interferers model, $R=200 \mathrm{~m}$

5. $A S E$ for conventional single tier co-channel interferers model, $R=500 \mathrm{~m}$ [77]

6. $A S E$ for proposed multiple tier co-channel interferers model, $R=500 \mathrm{~m}$ 


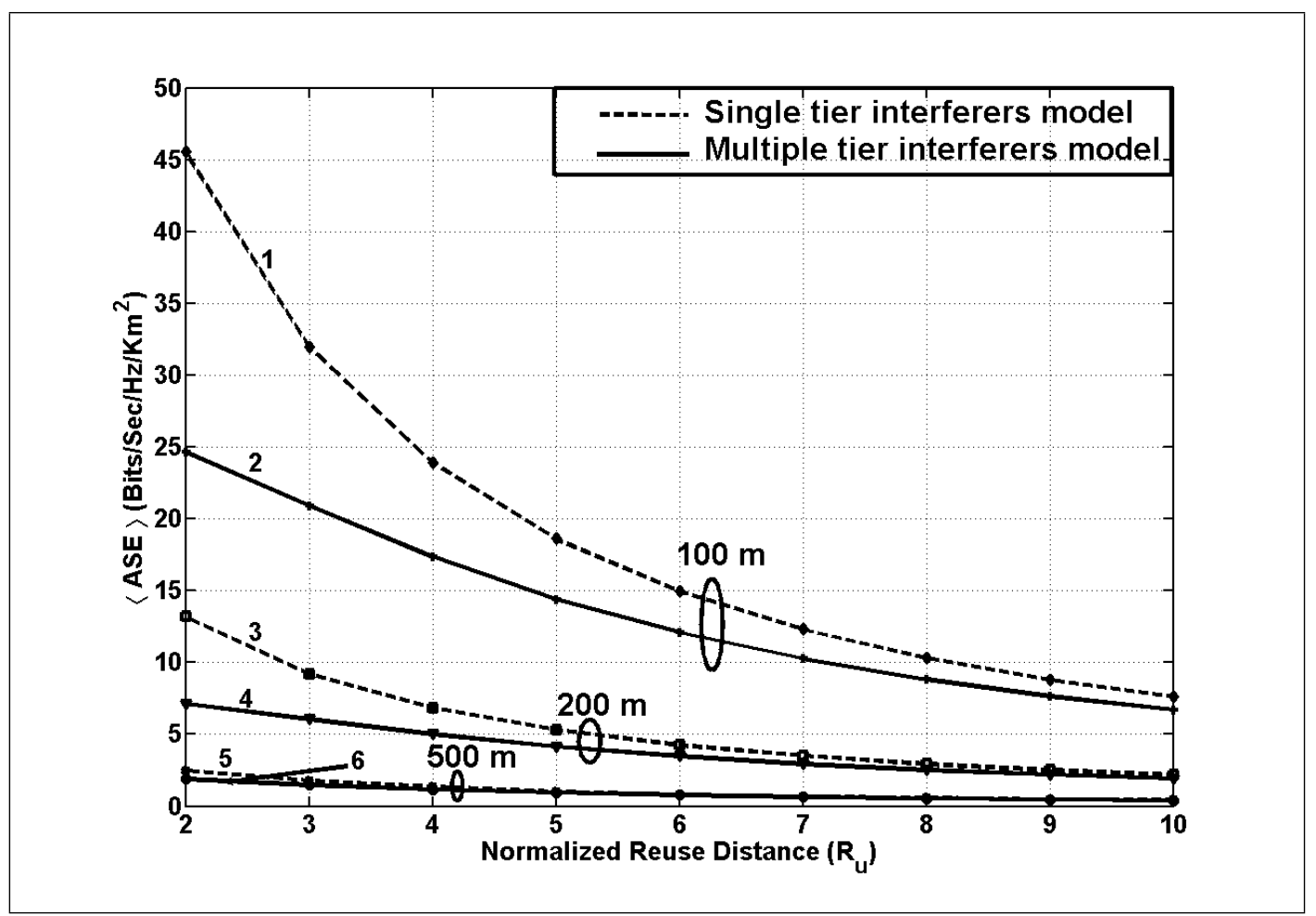

Figure 5.16: Comparison of the average area spectral efficiency, ASE for single and multiple tier interferers model for different normalized reuse distances $R_{u}$ and cell radius $R$. (Fully loaded non-sectorized cellular system; carrier frequency $f_{c}=8.45 \mathrm{GHz}$; path loss exponent $\alpha=2$ and extra path loss exponent $\rho=2$; BS antenna height $h_{b}=15 \mathrm{~m}$ and MS antenna height $h_{m}=1.5 \mathrm{~m}$; number of co-channel interfering cells; single tier $=6$ and multiple tier $=330$ )

1. ASE for conventional single tier co-channel interferers model, $R=100 \mathrm{~m}$ [77]

2. $A S E$ for proposed multiple tier co-channel interferers model, $R=100 \mathrm{~m}$

3. ASE for conventional single tier co-channel interferers model, $R=200 \mathrm{~m}$ [77]

4. $A S E$ for proposed multiple tier co-channel interferers model, $R=200 \mathrm{~m}$

5. $A S E$ for conventional single tier co-channel interferers model, $R=500 \mathrm{~m}$ [77]

6. $A S E$ for proposed multiple tier co-channel interferers model, $R=500 \mathrm{~m}$ 


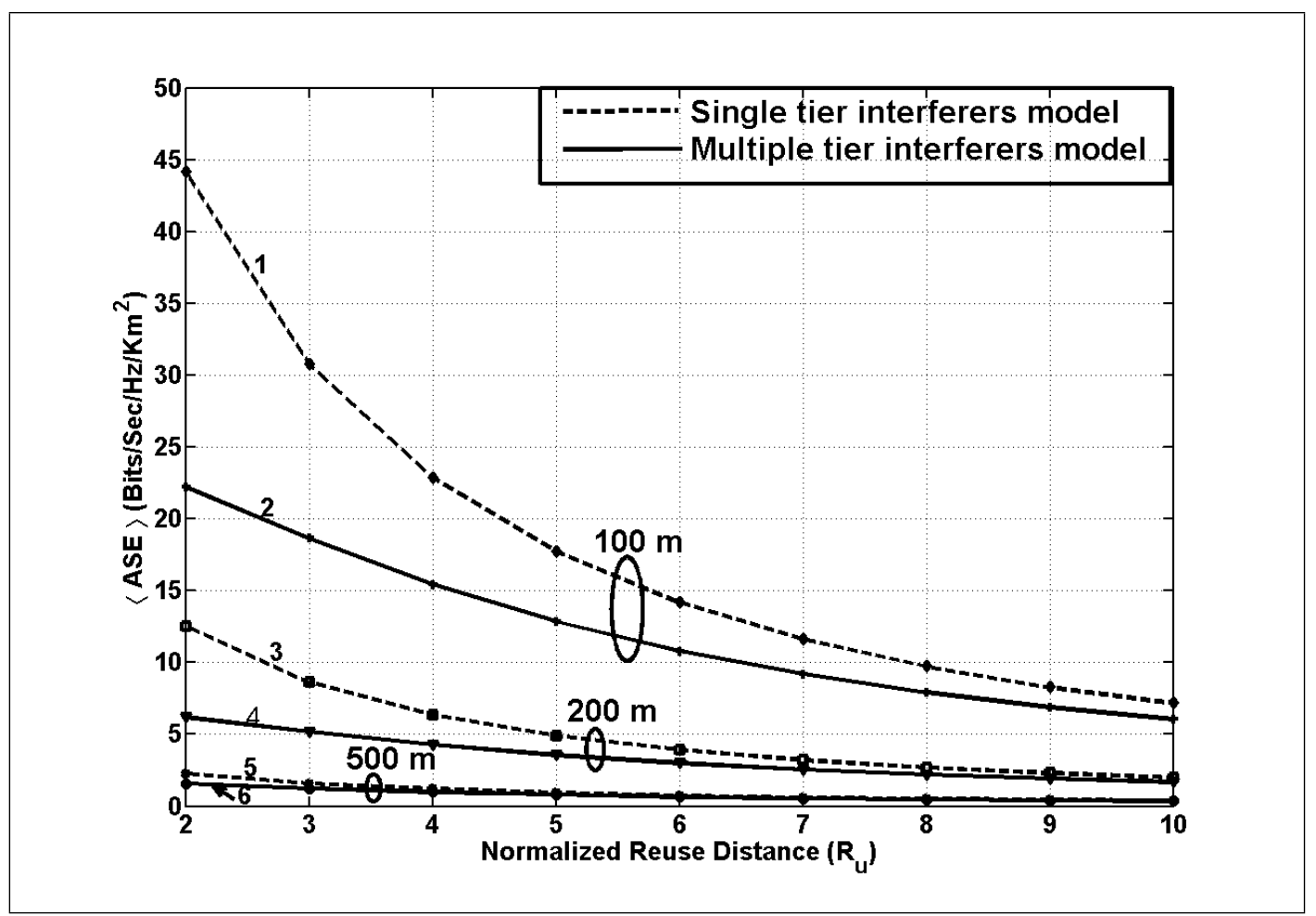

Figure 5.17: Comparison of the average area spectral efficiency, ASE for single and multiple tier interferers model for different normalized reuse distances $R_{u}$ and cell radius $R$. (Fully loaded non-sectorized cellular system; carrier frequency $f_{c}=15.75 \mathrm{GHz}$; path loss exponent $\alpha=2$ and extra path loss exponent $\rho=2$; BS antenna height $h_{b}=15 \mathrm{~m}$ and MS antenna height $h_{m}=1.5 \mathrm{~m}$; number of co-channel interfering cells; single tier $=6$ and multiple tier $=330$ )

1. ASE for conventional single tier co-channel interferers model, $R=100 \mathrm{~m}$ [77]

2. $A S E$ for proposed multiple tier co-channel interferers model, $R=100 \mathrm{~m}$

3. $A S E$ for conventional single tier co-channel interferers model, $R=200 \mathrm{~m}$ [77]

4. $A S E$ for proposed multiple tier co-channel interferers model, $R=200 \mathrm{~m}$

5. $A S E$ for conventional single tier co-channel interferers model, $R=500 \mathrm{~m}$ [77]

6. $A S E$ for proposed multiple tier co-channel interferers model, $R=500 \mathrm{~m}$ 


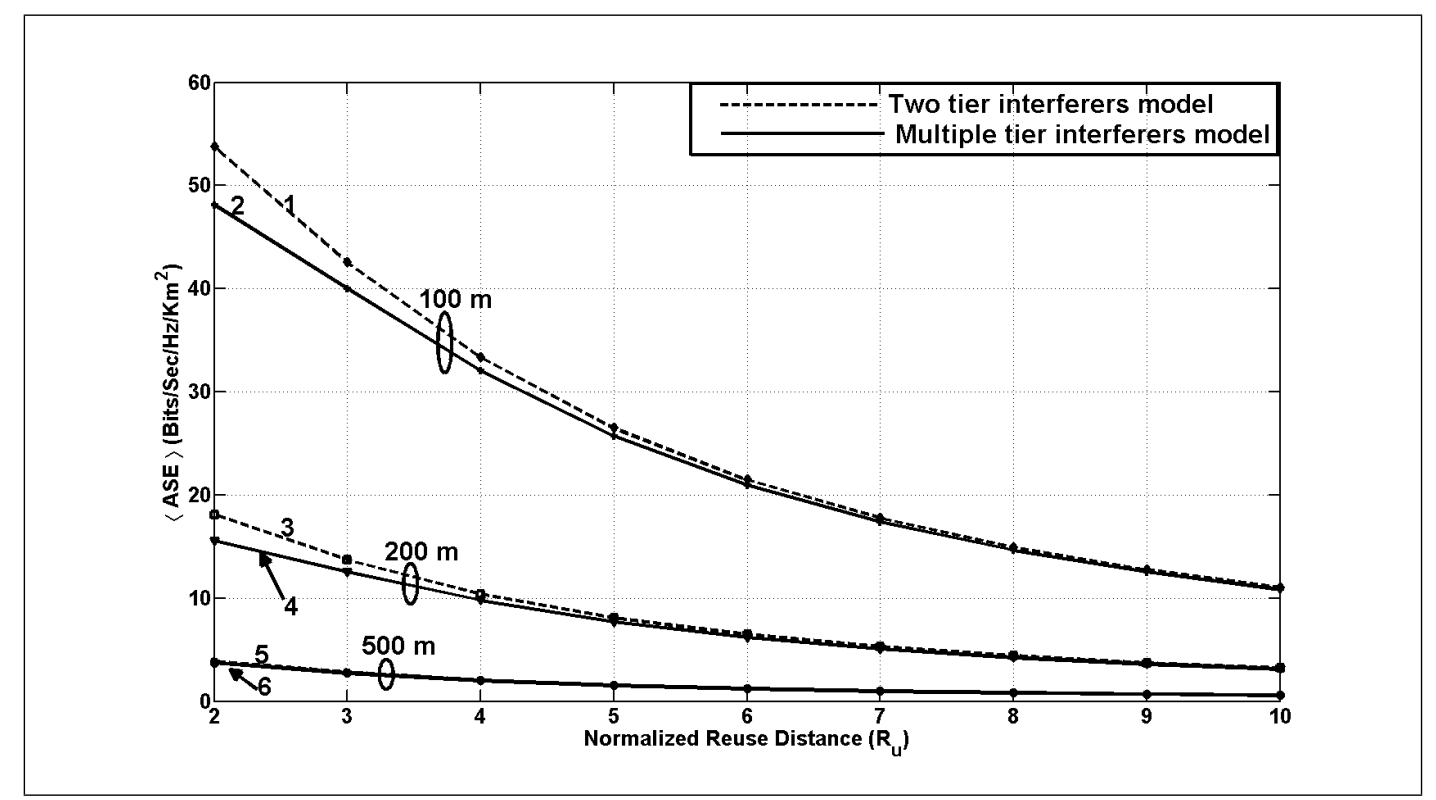

Figure 5.18: Comparison of the average area spectral efficiency, $A S E$ for two and multiple tier interferers model for different normalized reuse distances $R_{u}$ and cell radius $R$. (Fully loaded non-sectorized cellular system; carrier frequency $f_{c}=900 \mathrm{MHz}$; path loss exponent $\alpha=2$ and extra path loss exponent $\rho=2$; BS antenna height $h_{b}=15 \mathrm{~m}$ and MS antenna height $h_{m}=1.5 \mathrm{~m}$; number of co-channel interfering cells; two tier $=18$ and multiple tier $=330$.

1. $A S E$ for conventional single tier co-channel interferers model, $R=100 \mathrm{~m} \mathrm{[77]}$

2. $A S E$ for proposed multiple tier co-channel interferers model, $R=100 \mathrm{~m}$

3. $A S E$ for conventional single tier co-channel interferers model, $R=200 \mathrm{~m}$ [77]

4. $A S E$ for proposed multiple tier co-channel interferers model, $R=200 \mathrm{~m}$

5. $A S E$ for conventional single tier co-channel interferers model, $R=500 \mathrm{~m} \mathrm{[77]}$

6. ASE for proposed multiple tier co-channel interferers model, $R=500 \mathrm{~m}$ 


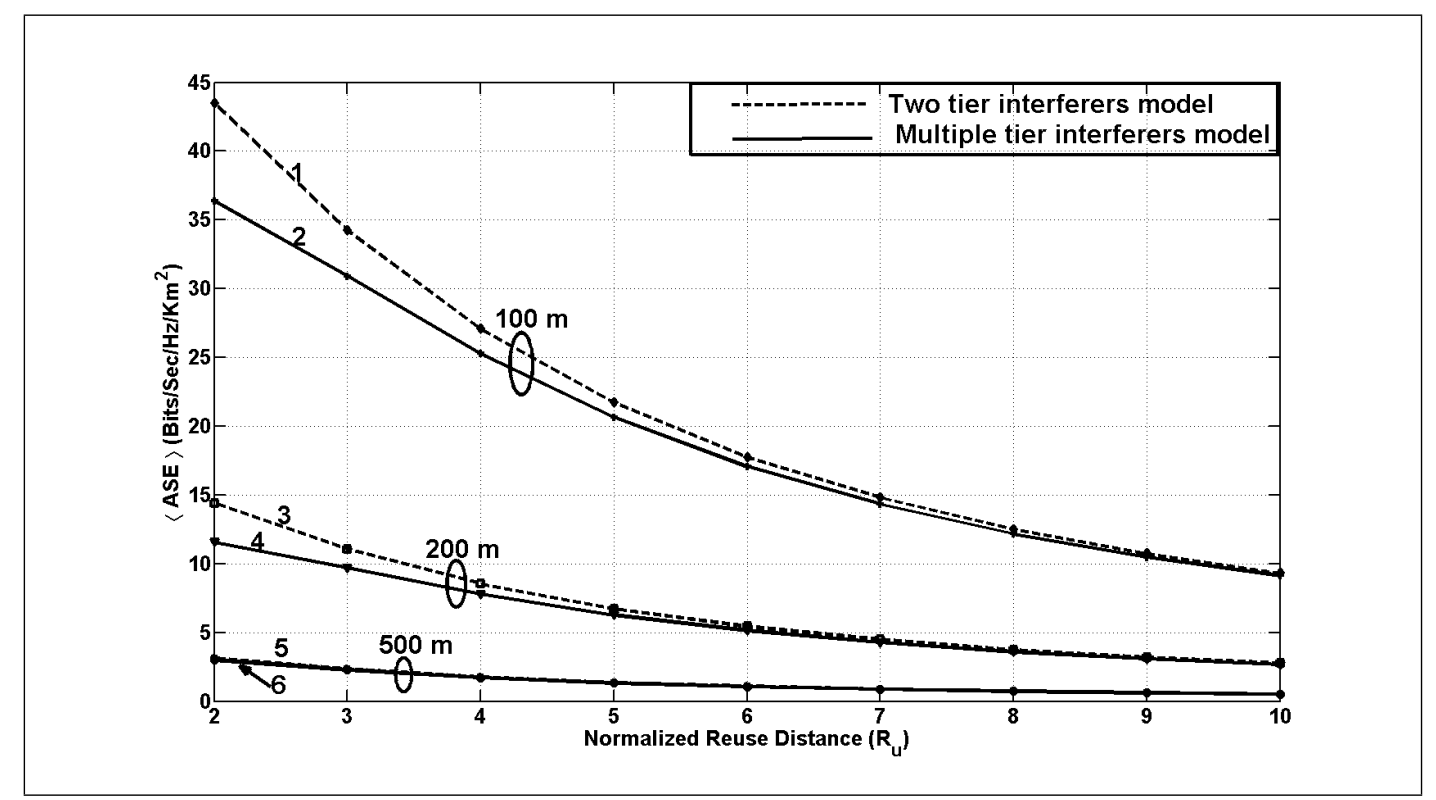

Figure 5.19: Comparison of the average area spectral efficiency, $A S E$ for two and multiple tier interferers model for different normalized reuse distances $R_{u}$ and cell radius $R$. (Fully loaded non-sectorized cellular system; carrier frequency $f_{c}=2 \mathrm{GHz}$; path loss exponent $\alpha=2$ and extra path loss exponent $\rho=2$; BS antenna height $h_{b}=15 \mathrm{~m}$ and MS antenna height $h_{m}=1.5 \mathrm{~m}$; number of co-channel interfering cells; two tier $=18$ and multiple tier $=330)$

1. $A S E$ for conventional single tier co-channel interferers model, $R=100 \mathrm{~m} \mathrm{[77]}$

2. $A S E$ for proposed multiple tier co-channel interferers model, $R=100 \mathrm{~m}$

3. $A S E$ for conventional single tier co-channel interferers model, $R=200 \mathrm{~m} \mathrm{[77]}$

4. $A S E$ for proposed multiple tier co-channel interferers model, $R=200 \mathrm{~m}$

5. $A S E$ for conventional single tier co-channel interferers model, $R=500 \mathrm{~m} \mathrm{[77]}$

6. $A S E$ for proposed multiple tier co-channel interferers model, $R=500 \mathrm{~m}$ 


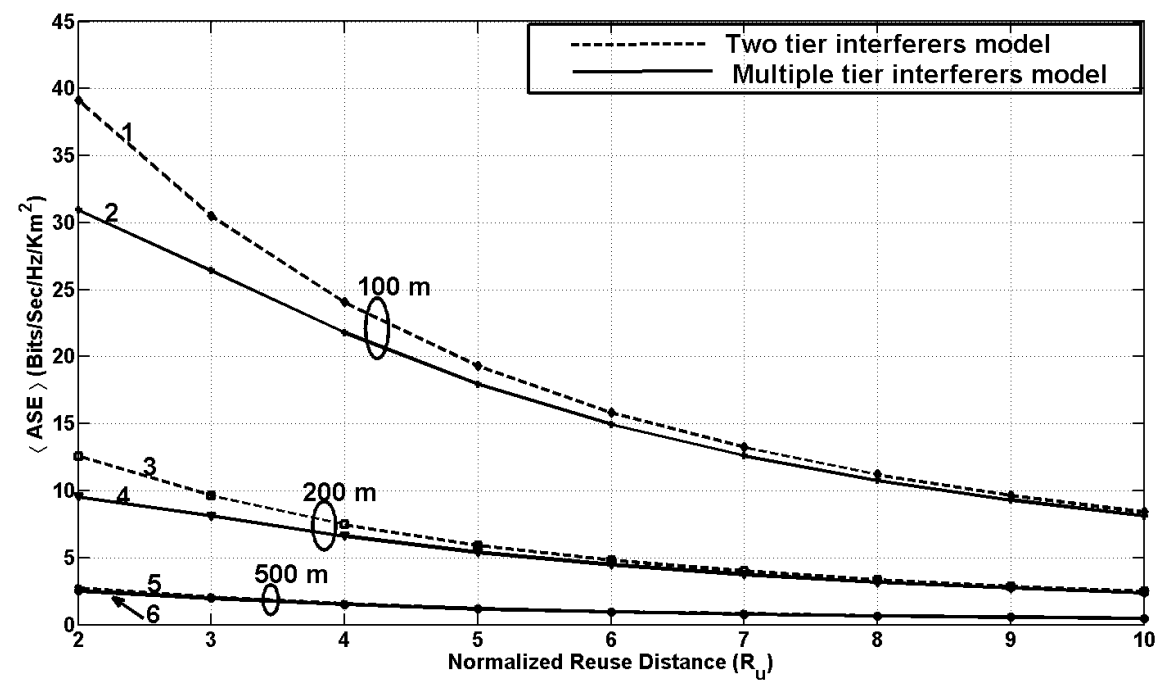

Figure 5.20: Comparison of the average area spectral efficiency, ASE for two and multiple tier interferers model for different normalized reuse distances $R_{u}$ and cell radius $R$. (Fully loaded non-sectorized cellular system; carrier frequency $f_{c}=3.35 \mathrm{GHz}$; path loss exponent $\alpha=2$ and extra path loss exponent $\rho=2$; BS antenna height $h_{b}=15 \mathrm{~m}$ and MS antenna height $h_{m}=1.5 \mathrm{~m}$; number of co-channel interfering cells; two tier $=18$ and multiple tier $=330)$

1. ASE for conventional single tier co-channel interferers model, $R=100 \mathrm{~m} \mathrm{[77]}$

2. $A S E$ for proposed multiple tier co-channel interferers model, $R=100 \mathrm{~m}$

3. $A S E$ for conventional single tier co-channel interferers model, $R=200 \mathrm{~m}$ [77]

4. $A S E$ for proposed multiple tier co-channel interferers model, $R=200 \mathrm{~m}$

5. $A S E$ for conventional single tier co-channel interferers model, $R=500 \mathrm{~m} \mathrm{[77]}$

6. $A S E$ for proposed multiple tier co-channel interferers model, $R=500 \mathrm{~m}$ 


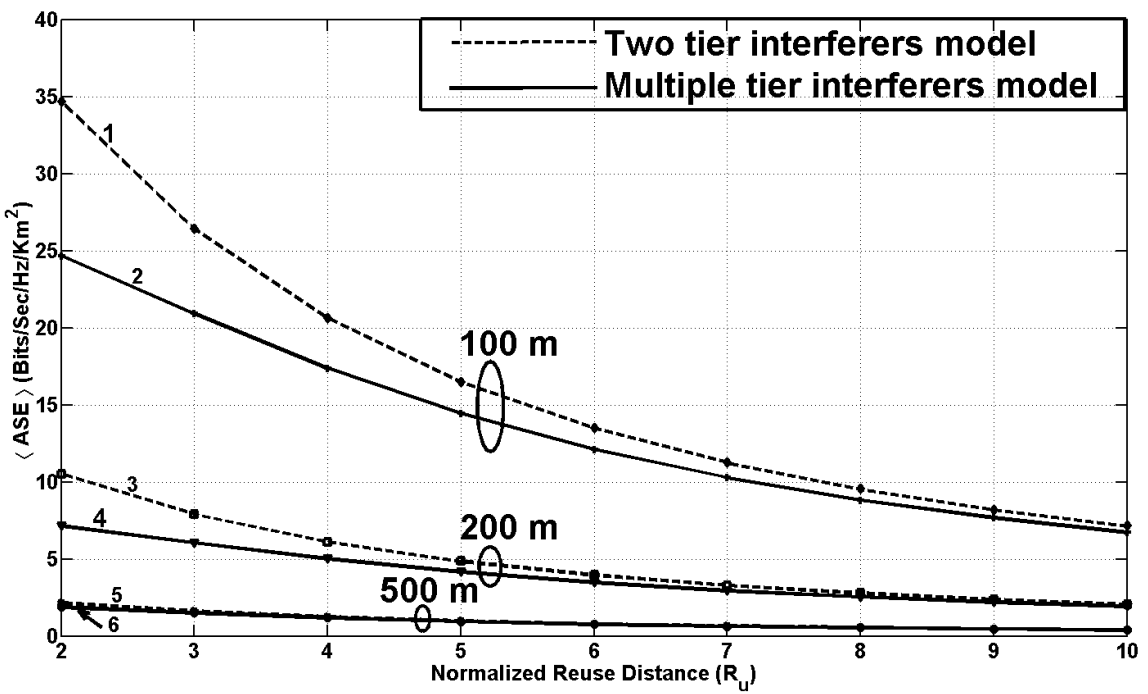

Figure 5.21: Comparison of the average area spectral efficiency, $A S E$ for two and multiple tier interferers model for different normalized reuse distances $R_{u}$ and cell radius $R$. (Fully loaded non-sectorized cellular system; carrier frequency $f_{c}=8.45 \mathrm{GHz}$; path loss exponent $\alpha=2$ and extra path loss exponent $\rho=2$; BS antenna height $h_{b}=15 \mathrm{~m}$ and MS antenna height $h_{m}=1.5 \mathrm{~m}$; number of co-channel interfering cells; two tier $=18$ and multiple tier $=330)$

1. $A S E$ for conventional single tier co-channel interferers model, $R=100 \mathrm{~m}$ [77]

2. $A S E$ for proposed multiple tier co-channel interferers model, $R=100 \mathrm{~m}$

3. $A S E$ for conventional single tier co-channel interferers model, $R=200 \mathrm{~m} \mathrm{[77]}$

4. $A S E$ for proposed multiple tier co-channel interferers model, $R=200 \mathrm{~m}$

5. ASE for conventional single tier co-channel interferers model, $R=500 \mathrm{~m}$ [77]

6. $A S E$ for proposed multiple tier co-channel interferers model, $R=500 \mathrm{~m}$ 


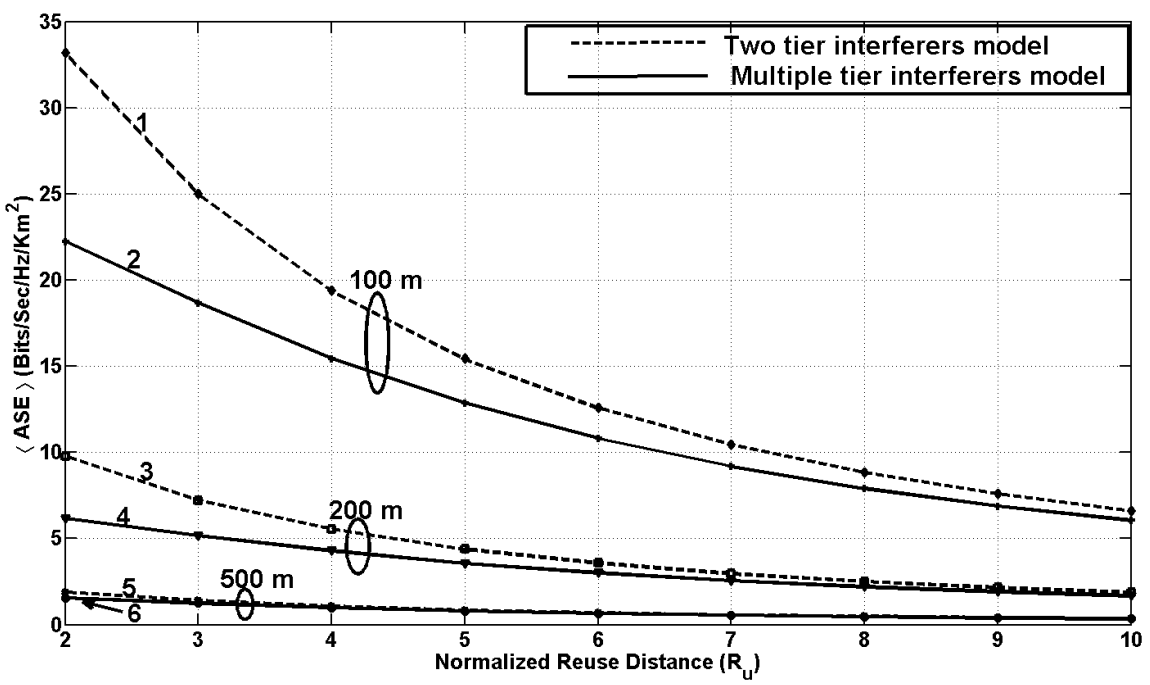

Figure 5.22: Comparison of the average area spectral efficiency, ASE for two and multiple tier interferers model for different normalized reuse distances $R_{u}$ and cell radius $R$. (Fully loaded non-sectorized cellular system; carrier frequency $f_{c}=15.75 \mathrm{GHz}$; path loss exponent $\alpha=2$ and extra path loss exponent $\rho=2$; BS antenna height $h_{b}=15 \mathrm{~m}$ and MS antenna height $h_{m}=1.5 \mathrm{~m}$; number of co-channel interfering cells; two tier $=18$ and multiple tier $=330$ )

1. ASE for conventional single tier co-channel interferers model, $R=100 \mathrm{~m}$ [77]

2. $A S E$ for proposed multiple tier co-channel interferers model, $R=100 \mathrm{~m}$

3. $A S E$ for conventional single tier co-channel interferers model, $R=200 \mathrm{~m}$ [77]

4. $A S E$ for proposed multiple tier co-channel interferers model, $R=200 \mathrm{~m}$

5. $A S E$ for conventional single tier co-channel interferers model, $R=500 \mathrm{~m} \mathrm{[77]}$

6. $A S E$ for proposed multiple tier co-channel interferers model, $R=500 \mathrm{~m}$ 


\subsubsection{Impact of Basic Path Loss Exponent-Multiple Tier Interference}

Figures 5.23 - 5.26, show the simulation results for different basic path loss exponent $\alpha$. This is for the study of the impact of propagation loss parameters at higher carrier frequencies, and small cell size radius, when multiple tiers of interference are active. Figure 5.23, compares the information capacity for the single and multiple tier co-channel interference models for different carrier frequencies $f_{c}$, when basic path loss exponent $\alpha=2$. The curves show that when $f_{c}=900 \mathrm{MHz}$, and $R=0.1 \mathrm{~km}$, there is a $5.67 \%$ decrease in information capacity between the single and multiple tier interferers model. At $0.3 \mathrm{~km}$, the decrease is $1.47 \%$, and still less for greater values of $R$. For $f_{c}=2 \mathrm{GHz}$ at $0.1 \mathrm{~km}$, the decrease in $A S E$ was $10.45 \%$. At $0.3 \mathrm{~km}$, it was $2.46 \%$, and less still for greater values of $R$. For $3.35 \mathrm{GHz}$, the decrease was $15.60 \%$ and $3.78 \%$, at 0.1 and $0.3 \mathrm{~km}$. Now for $8.45 \mathrm{GHz}$ the decrease in $A S E$ was $27.86 \%$ at $0.1 \mathrm{~km}, 9.05 \%$ at $0.3 \mathrm{~km}, 4.13 \%$ at $0.6 \mathrm{~km}$, $2.74 \%$ at $0.8 \mathrm{~km}$, and less still for greater values of $R$. For $15.75 \mathrm{GHz}$ the decrease at 0.1 , $0.3,0.6$ and $0.8 \mathrm{~km}$ was $36.41,15.27,7.44$ and $5.45 \%$.

Figure 5.24, compares the $A S E$ for the two interference models, when $\alpha=2.5$. At $f_{c}=900 \mathrm{MHz}$ and $0.1 \mathrm{~km}$, the decrease was $3.78 \%$, and less for greater values of $R$. For $2 \mathrm{GHz}$, at $0.1 \mathrm{~km}$, the decrease was $6.88 \%$. At $0.3 \mathrm{~km}$ it was $1.43 \%$, and less for greater values of $R$. For $f_{c}=3.35 \mathrm{GHz}$, the decrease was $10.00 \%$ and $2.23 \%$ at 0.1 and $0.3 \mathrm{~km}$. At $8.45 \mathrm{GHz}$ and $0.1 \mathrm{~km}$ the decrease in $A S E$ was $17.69 \%$ at $0.3 \mathrm{~km}$ it was $5.26 \%$ and at $0.6 \mathrm{~km}$ was $2.86 \%$ and less for greater values of $R$. For $15.75 \mathrm{GHz}$, the decrease at 0.1 , 0.3 and $0.6 \mathrm{~km}$, was $23.17,8.69$ and $4.27 \%$.

Figure 5.25, compares the $A S E$ for the two interference models, when $\alpha=2.8$. For $f_{c}=900 \mathrm{MHz}, 2 \mathrm{GHz}$ and $3.35 \mathrm{GHz}$, the curves show a lesser decrease in information capacity between the two interference models. For $8.45 \mathrm{GHz}$, at $0.1 \mathrm{~km}$, there was $13.40 \%$ decrease in $A S E$. At $0.3 \mathrm{~km}$, it was $3.85 \%$. For $15.75 \mathrm{GHz}$, the decrease at $0.1,0.3 \mathrm{~km}$, was 17.52 and $6.27 \%$.

Figure 5.26, compares the $A S E$ for the two interference models, when $\alpha=3$. For $f_{c}=900 \mathrm{MHz}, 2 \mathrm{GHz}$ and $3.35 \mathrm{GHz}$, at $R=0.1 \mathrm{~km}$, the decrease in ASE between the two models was $2.60 \%, 4.51 \%$ and $6.49 \%$. It was however, less than $1.5 \%$ at $R=0.3 \mathrm{~km}$. However, for $8.45 \mathrm{GHz}$, at $0.1 \mathrm{~km}$, there was $11.18 \%$ decrease in $A S E$. At $0.3 \mathrm{~km}$, the 
decrease was $3.2 \%$, and still less for greater values of $R$. For $15.75 \mathrm{GHz}$, the decrease at 0.1 and $0.3 \mathrm{~km}$, was $17.48 \%$ and $6.27 \%$.

Figures 5.27 - 5.30, compares the ASE for two and multiple tier interference models. The information capacity comparison is for different basic path loss exponent $\alpha$, and carrier frequencies $f_{c}$. The curves show a lesser decrease in $A S E$ as carrier frequency decrease. The curves also show a lesser decrease in $A S E$ as the basic path loss exponent increases.

From the graphs we can conclude that for carrier frequencies greater than $2 \mathrm{GHz}$; at lower path loss exponent and smaller cell radius, multiple tiers of interference become active, which causes co-channel interference to be severe. This causes a decrease in the information capacity of a cellular wireless communication system. The curves also show the impact of basic path loss exponent on the information capacity performance of a cellular wireless network, operating at higher carrier frequencies and having a smaller cell size radius. 


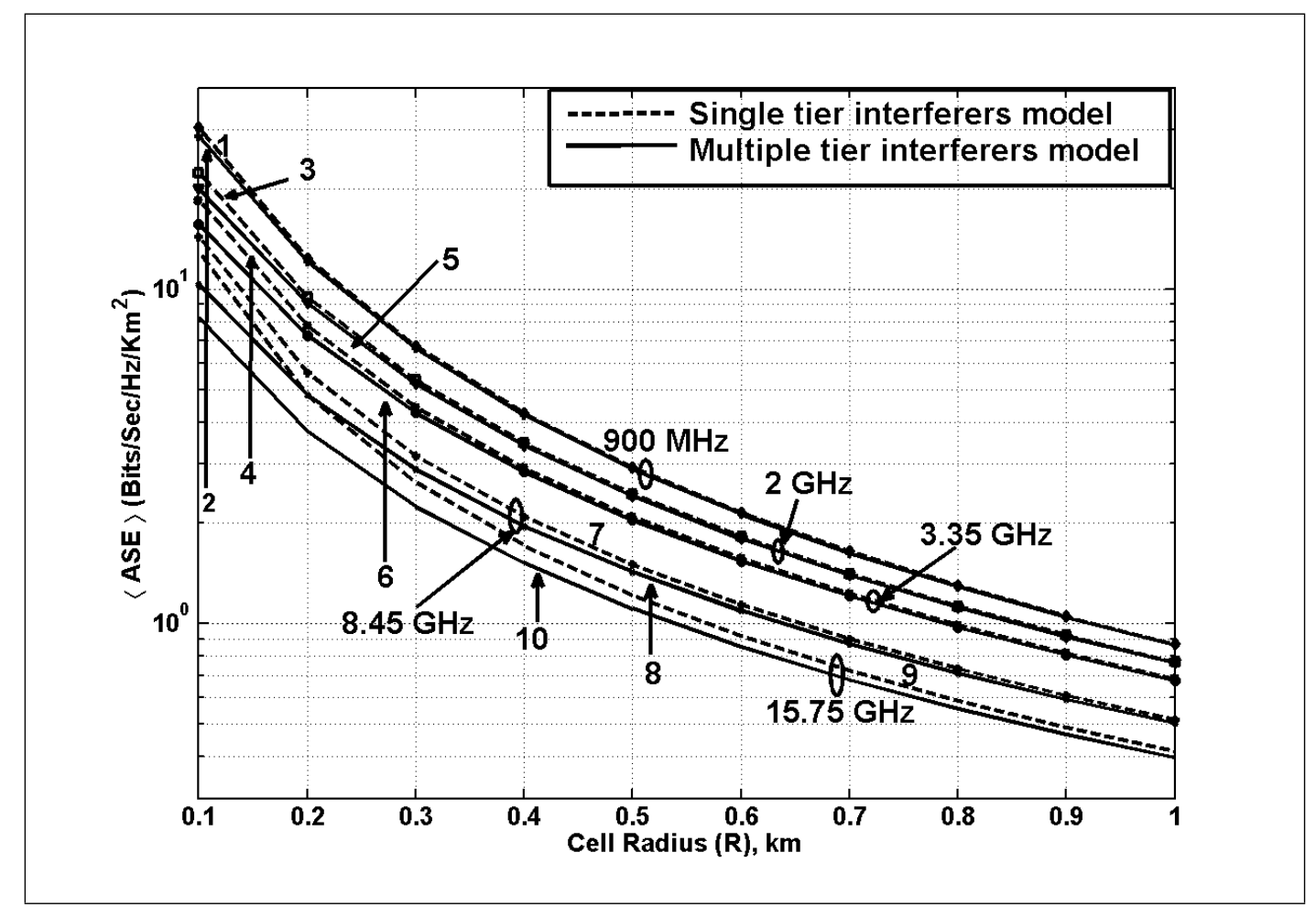

Figure 5.23: Average uplink area spectral efficiency versus cell radius for path loss exponent, $\alpha=2$, and different carrier frequencies $f_{c}$. (Fully-loaded cellular system with co-channel interfering cells in first tier, $N_{F I}=6$, and multiple tier, $N_{N I}=330$; extra path loss exponent, $\rho=4$; MS and BS antenna heights : $h_{m}=1.5 \mathrm{~m}$ and $h_{b}=15 \mathrm{~m}$ )

1 Single tier co-channel interfering model $\left(f_{c}=900 \mathrm{MHz}\right)[77]$

2 Multiple tier co-channel interfering model $\left(f_{c}=900 \mathrm{MHz}\right)$

3 Single tier co-channel interfering model $\left(f_{c}=2 \mathrm{GHz}\right)[77]$

4 Multiple tier co-channel interfering model $\left(f_{c}=2 \mathrm{GHz}\right)$

5 Single tier co-channel interfering model $\left(f_{c}=3.35 \mathrm{GHz}\right)[77]$

6 Multiple tier co-channel interfering model $\left(f_{c}=3.35 \mathrm{GHz}\right)$

7 Single tier co-channel interfering model $\left(f_{c}=8.45 \mathrm{GHz}\right)[77]$

8 Multiple tier co-channel interfering model $\left(f_{c}=8.45 \mathrm{GHz}\right)$

9 Single tier co-channel interfering model $\left(f_{c}=15.75 \mathrm{GHz}\right)$ [77]

10 Multiple tier co-channel interfering model $\left(f_{c}=15.75 \mathrm{GHz}\right)$ 


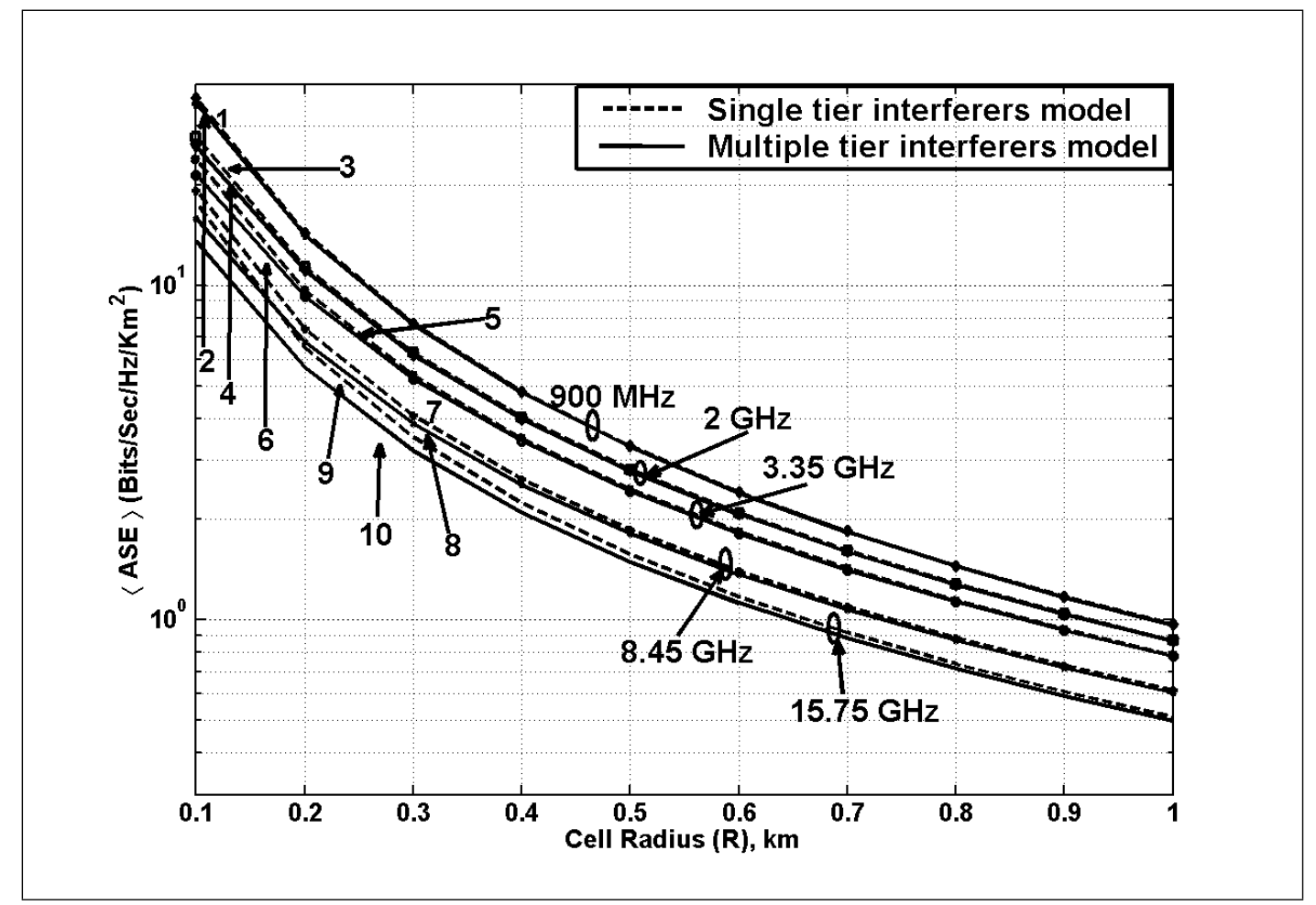

Figure 5.24: Average uplink area spectral efficiency versus cell radius for path loss exponent, $\alpha=2.5$, and different carrier frequencies $f_{c}$. (Fully-loaded cellular system with co-channel interfering cells in first tier, $N_{F I}=6$, and multiple tier, $N_{N I}=330$; extra path loss exponent, $\rho=4$; MS and BS antenna heights : $h_{m}=1.5 \mathrm{~m}$ and $h_{b}=15 \mathrm{~m}$ )

1 Single tier co-channel interfering model $\left(f_{c}=900 \mathrm{MHz}\right)$ [77]

2 Multiple tier co-channel interfering model $\left(f_{c}=900 \mathrm{MHz}\right)$

3 Single tier co-channel interfering model $\left(f_{c}=2 \mathrm{GHz}\right)[77]$

4 Multiple tier co-channel interfering model $\left(f_{c}=2 \mathrm{GHz}\right)$

5 Single tier co-channel interfering model $\left(f_{c}=3.35 \mathrm{GHz}\right)[77]$

6 Multiple tier co-channel interfering model $\left(f_{c}=3.35 \mathrm{GHz}\right)$

7 Single tier co-channel interfering model $\left(f_{c}=8.45 \mathrm{GHz}\right)[77]$

8 Multiple tier co-channel interfering model $\left(f_{c}=8.45 \mathrm{GHz}\right)$

9 Single tier co-channel interfering model $\left(f_{c}=15.75 \mathrm{GHz}\right)$ [77]

10 Multiple tier co-channel interfering model $\left(f_{c}=15.75 \mathrm{GHz}\right)$ 


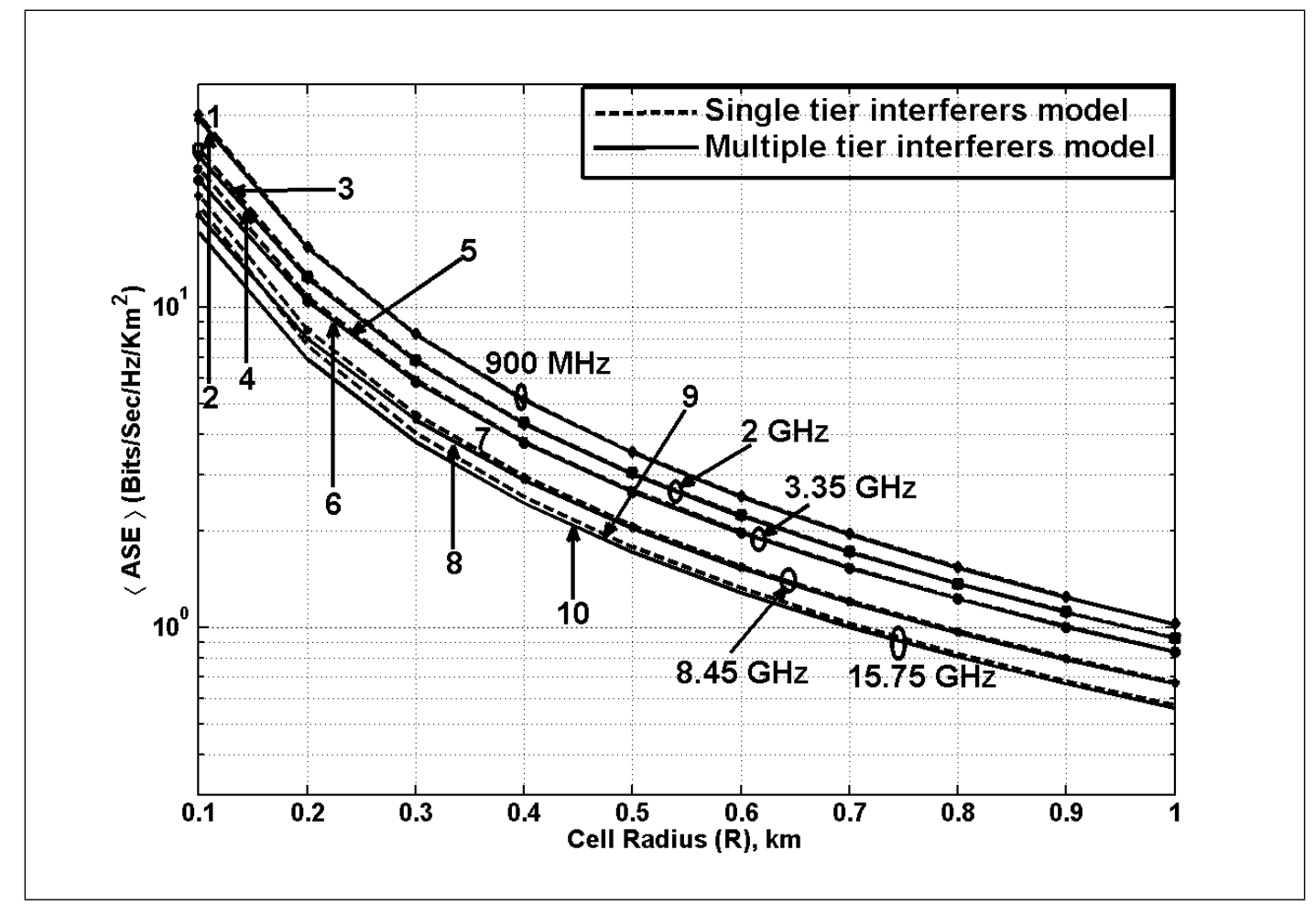

Figure 5.25: Average uplink area spectral efficiency versus cell radius for path loss exponent, $\alpha=2.8$, and different carrier frequencies $f_{c}$. (Fully-loaded cellular system with co-channel interfering cells in first tier, $N_{F I}=6$, and multiple tier, $N_{N I}=330$; extra path loss exponent, $\rho=4$; MS and BS antenna heights : $h_{m}=1.5 \mathrm{~m}$ and $h_{b}=15 \mathrm{~m}$ )

1 Single tier co-channel interfering model $\left(f_{c}=900 \mathrm{MHz}\right)$ [77]

2 Multiple tier co-channel interfering model $\left(f_{c}=900 \mathrm{MHz}\right)$

3 Single tier co-channel interfering model $\left(f_{c}=2 \mathrm{GHz}\right)[77]$

4 Multiple tier co-channel interfering model $\left(f_{c}=2 \mathrm{GHz}\right)$

5 Single tier co-channel interfering model $\left(f_{c}=3.35 \mathrm{GHz}\right)$ [77]

6 Multiple tier co-channel interfering model $\left(f_{c}=3.35 \mathrm{GHz}\right)$

7 Single tier co-channel interfering model $\left(f_{c}=8.45 \mathrm{GHz}\right)[77]$

8 Multiple tier co-channel interfering model $\left(f_{c}=8.45 \mathrm{GHz}\right)$

9 Single tier co-channel interfering model $\left(f_{c}=15.75 \mathrm{GHz}\right)$ [77]

10 Multiple tier co-channel interfering model $\left(f_{c}=15.75 \mathrm{GHz}\right)$ 


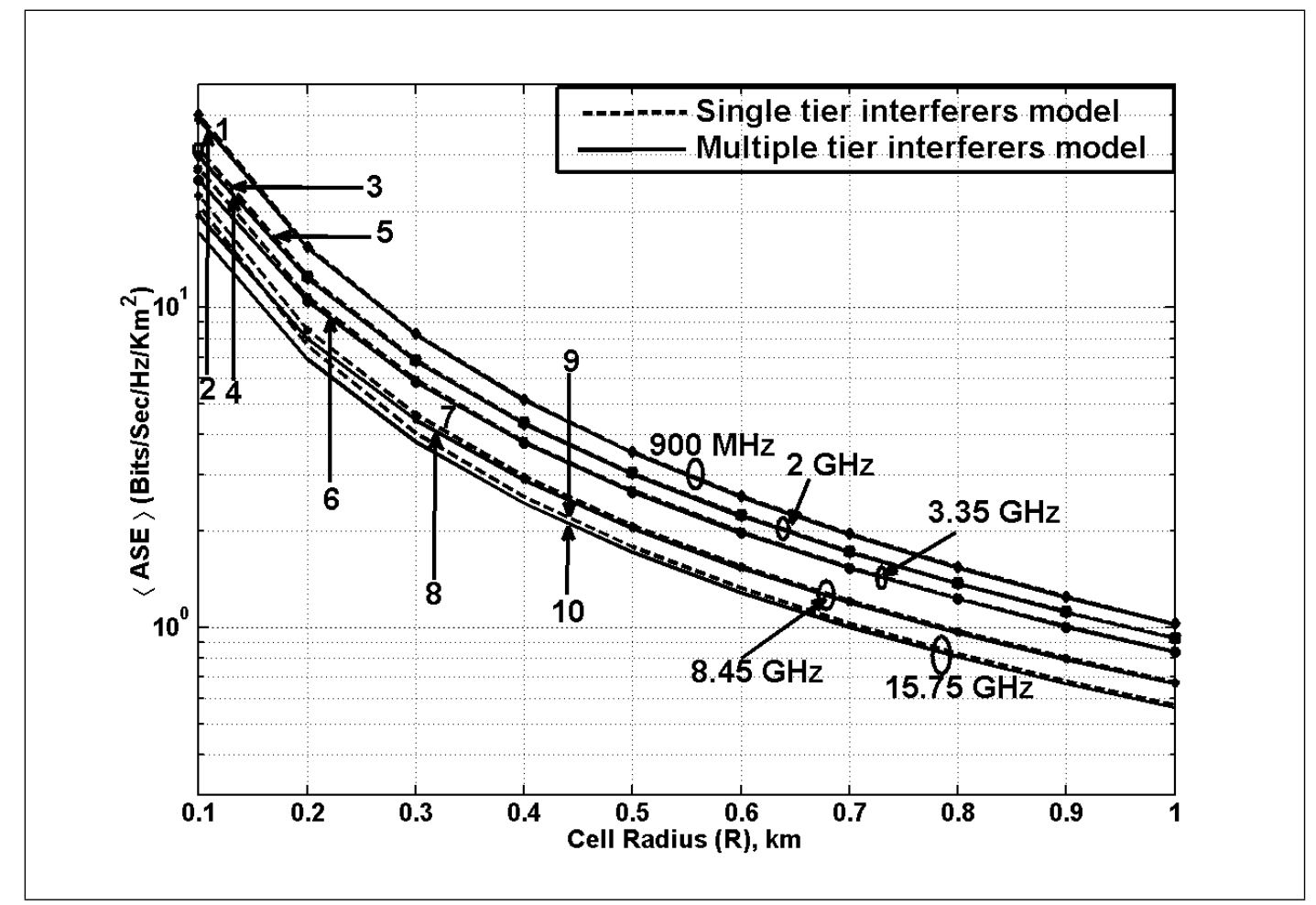

Figure 5.26: Average uplink area spectral efficiency versus cell radius for path loss exponent, $\alpha=3$, and different carrier frequencies $f_{c}$. (Fully-loaded cellular system with co-channel interfering cells in first tier, $N_{F I}=6$, and multiple tier, $N_{N I}=330$; extra path loss exponent, $\rho=4$; MS and BS antenna heights : $h_{m}=1.5 \mathrm{~m}$ and $h_{b}=15 \mathrm{~m}$ )

1 Single tier co-channel interfering model $\left(f_{c}=900 \mathrm{MHz}\right)[77]$

2 Multiple tier co-channel interfering model $\left(f_{c}=900 \mathrm{MHz}\right)$

3 Single tier co-channel interfering model $\left(f_{c}=2 \mathrm{GHz}\right)[77]$

4 Multiple tier co-channel interfering model $\left(f_{c}=2 \mathrm{GHz}\right)$

5 Single tier co-channel interfering model $\left(f_{c}=3.35 \mathrm{GHz}\right)[77]$

6 Multiple tier co-channel interfering model $\left(f_{c}=3.35 \mathrm{GHz}\right)$

7 Single tier co-channel interfering model $\left(f_{c}=8.45 \mathrm{GHz}\right)[77]$

8 Multiple tier co-channel interfering model $\left(f_{c}=8.45 \mathrm{GHz}\right)$

9 Single tier co-channel interfering model $\left(f_{c}=15.75 \mathrm{GHz}\right)$ [77]

10 Multiple tier co-channel interfering model $\left(f_{c}=15.75 \mathrm{GHz}\right)$ 


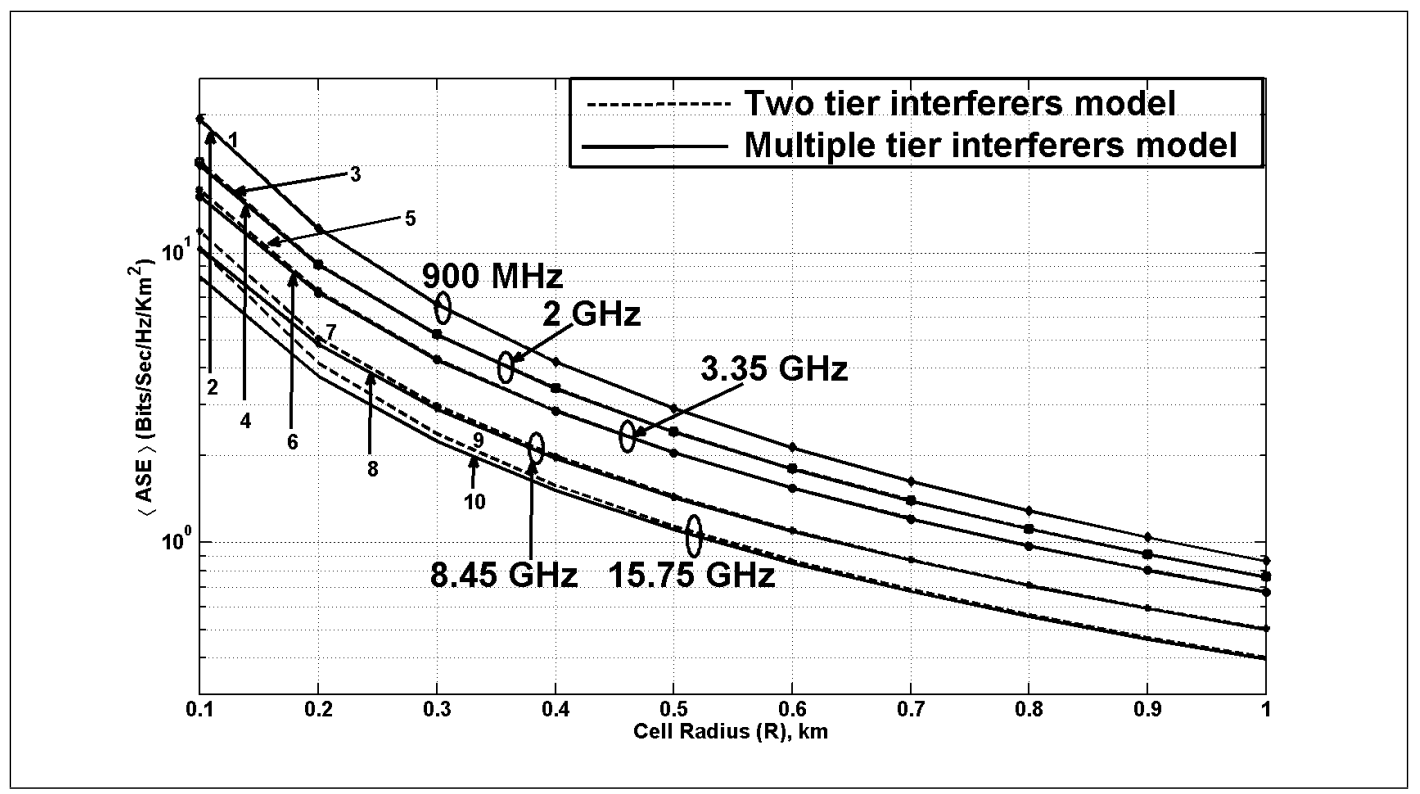

Figure 5.27: Average uplink area spectral efficiency versus cell radius for path loss exponent, $\alpha=2$, and different carrier frequencies $f_{c}$. (Fully-loaded cellular system with co-channel interfering cells in two tier, $N_{F I}=18$, and multiple tier, $N_{N I}=330$; extra path loss exponent, $\rho=4$; MS and BS antenna heights : $h_{m}=1.5 \mathrm{~m}$ and $h_{b}=15 \mathrm{~m}$ )

1 Single tier co-channel interfering model $\left(f_{c}=900 \mathrm{MHz}\right)$ [77]

2 Multiple tier co-channel interfering model $\left(f_{c}=900 \mathrm{MHz}\right)$

3 Single tier co-channel interfering model $\left(f_{c}=2 \mathrm{GHz}\right)$ [77]

4 Multiple tier co-channel interfering model $\left(f_{c}=2 \mathrm{GHz}\right)$

5 Single tier co-channel interfering model $\left(f_{c}=3.35 \mathrm{GHz}\right)[77]$

6 Multiple tier co-channel interfering model $\left(f_{c}=3.35 \mathrm{GHz}\right)$

7 Single tier co-channel interfering model $\left(f_{c}=8.45 \mathrm{GHz}\right)[77]$

8 Multiple tier co-channel interfering model $\left(f_{c}=8.45 \mathrm{GHz}\right)$

9 Single tier co-channel interfering model $\left(f_{c}=15.75 \mathrm{GHz}\right)[77]$

10 Multiple tier co-channel interfering model $\left(f_{c}=15.75 \mathrm{GHz}\right)$ 


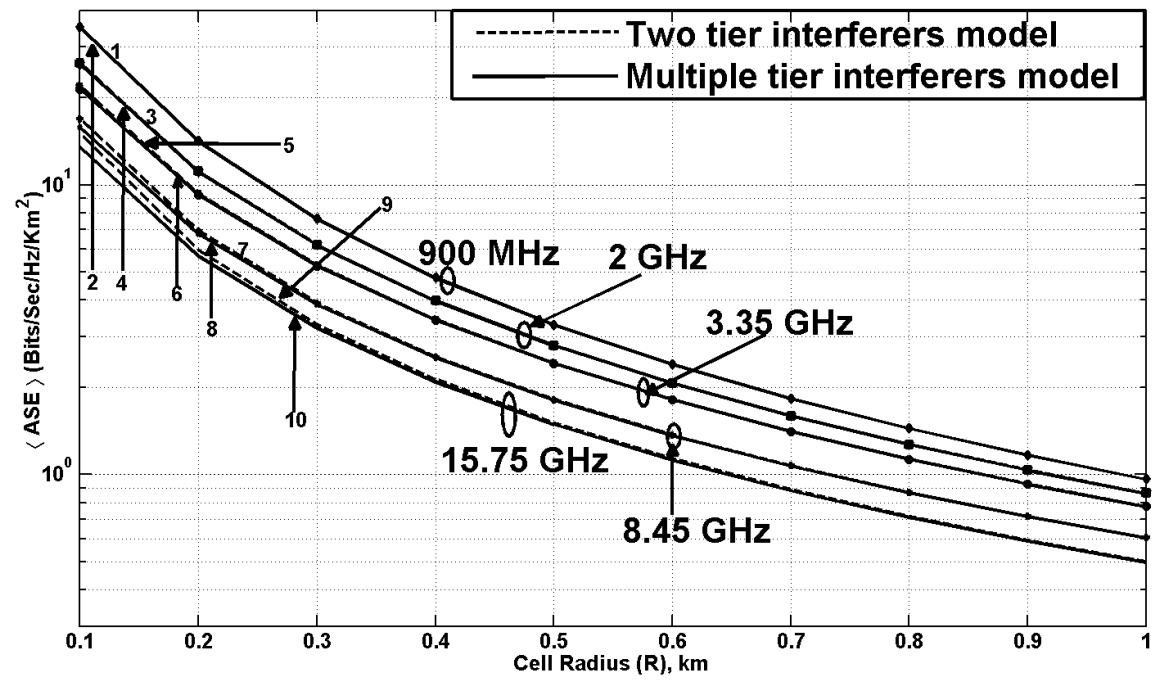

Figure 5.28: Average uplink area spectral efficiency versus cell radius for path loss exponent, $\alpha=2.5$, and different carrier frequencies $f_{c}$. (Fully-loaded cellular system with co-channel interfering cells in two tier, $N_{F I}=18$, and multiple tier, $N_{N I}=330$; extra path loss exponent, $\rho=4$; MS and BS antenna heights : $h_{m}=1.5 \mathrm{~m}$ and $h_{b}=15 \mathrm{~m}$ )

1 Single tier co-channel interfering model $\left(f_{c}=900 \mathrm{MHz}\right)$ [77]

2 Multiple tier co-channel interfering model $\left(f_{c}=900 \mathrm{MHz}\right)$

3 Single tier co-channel interfering model $\left(f_{c}=2 \mathrm{GHz}\right)$ [77]

4 Multiple tier co-channel interfering model $\left(f_{c}=2 \mathrm{GHz}\right)$

5 Single tier co-channel interfering model $\left(f_{c}=3.35 \mathrm{GHz}\right)[77]$

6 Multiple tier co-channel interfering model $\left(f_{c}=3.35 \mathrm{GHz}\right)$

7 Single tier co-channel interfering model $\left(f_{c}=8.45 \mathrm{GHz}\right)[77]$

8 Multiple tier co-channel interfering model $\left(f_{c}=8.45 \mathrm{GHz}\right)$

9 Single tier co-channel interfering model $\left(f_{c}=15.75 \mathrm{GHz}\right)$ [77]

10 Multiple tier co-channel interfering model $\left(f_{c}=15.75 \mathrm{GHz}\right)$ 


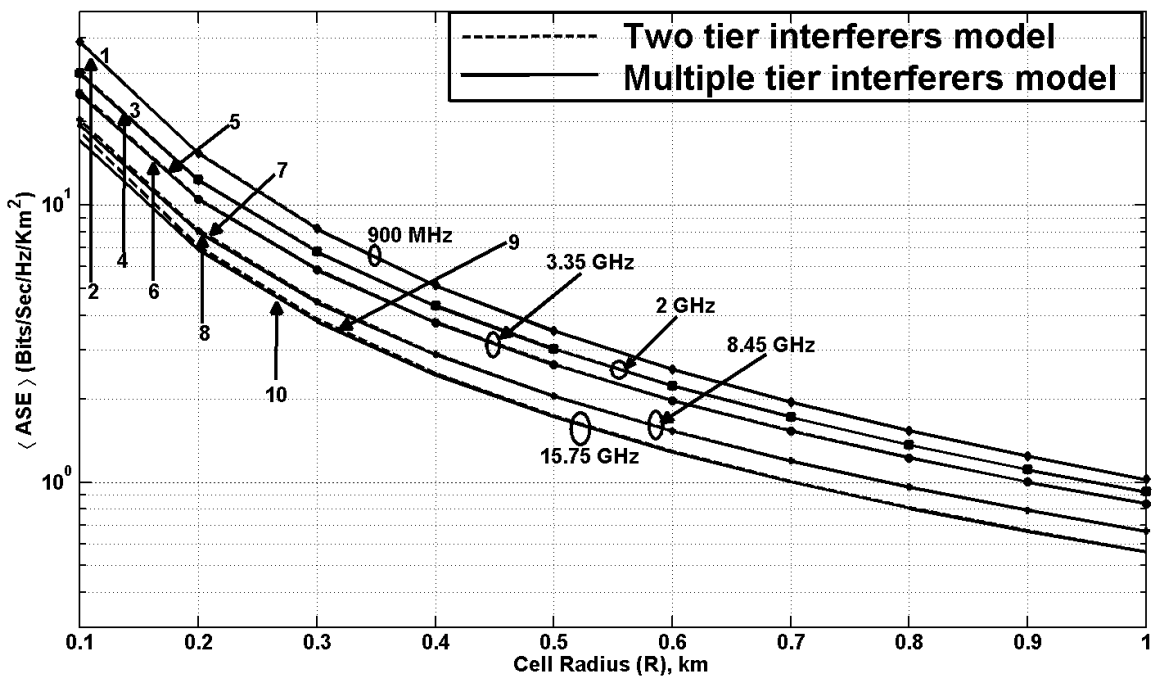

Figure 5.29: Average uplink area spectral efficiency versus cell radius for path loss exponent, $\alpha=2.8$, and different carrier frequencies $f_{c}$. (Fully-loaded cellular system with co-channel interfering cells in two tier, $N_{F I}=18$, and multiple tier, $N_{N I}=330$; extra path loss exponent, $\rho=4$; MS and BS antenna heights : $h_{m}=1.5 \mathrm{~m}$ and $h_{b}=15 \mathrm{~m}$ )

1 Single tier co-channel interfering model $\left(f_{c}=900 \mathrm{MHz}\right)$ [77]

2 Multiple tier co-channel interfering model $\left(f_{c}=900 \mathrm{MHz}\right)$

3 Single tier co-channel interfering model $\left(f_{c}=2 \mathrm{GHz}\right)$ [77]

4 Multiple tier co-channel interfering model $\left(f_{c}=2 \mathrm{GHz}\right)$

5 Single tier co-channel interfering model $\left(f_{c}=3.35 \mathrm{GHz}\right)[77]$

6 Multiple tier co-channel interfering model $\left(f_{c}=3.35 \mathrm{GHz}\right)$

7 Single tier co-channel interfering model $\left(f_{c}=8.45 \mathrm{GHz}\right)[77]$

8 Multiple tier co-channel interfering model $\left(f_{c}=8.45 \mathrm{GHz}\right)$

9 Single tier co-channel interfering model $\left(f_{c}=15.75 \mathrm{GHz}\right)$ [77]

10 Multiple tier co-channel interfering model $\left(f_{c}=15.75 \mathrm{GHz}\right)$ 


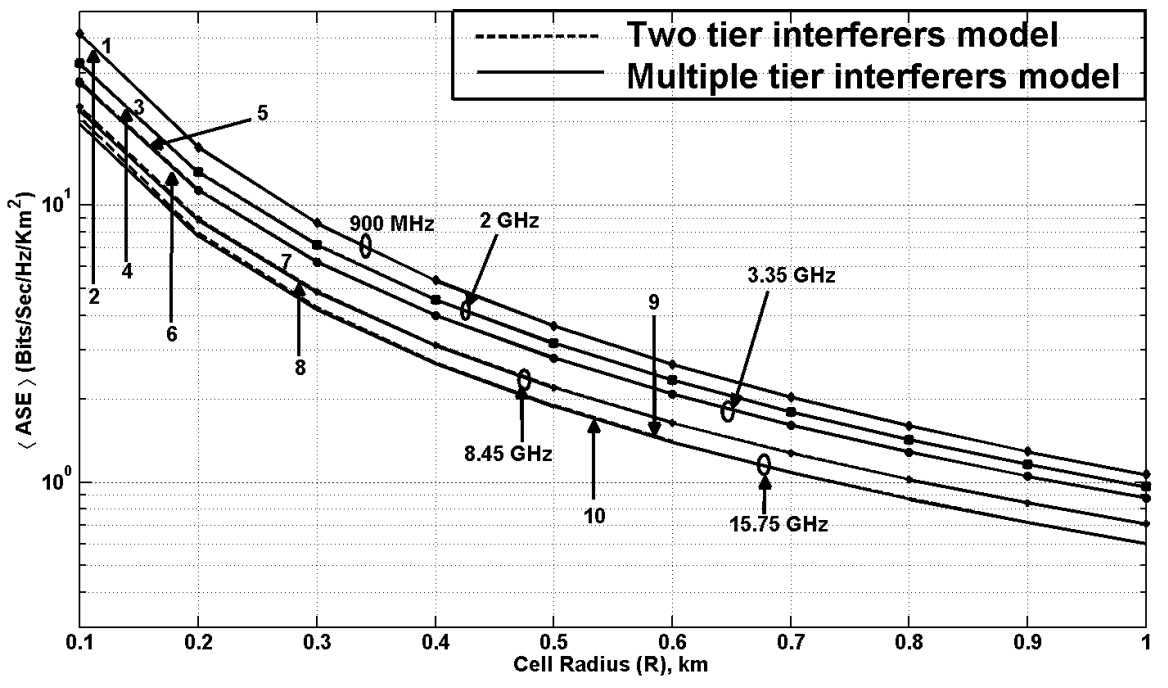

Figure 5.30: Average uplink area spectral efficiency versus cell radius for path loss exponent, $\alpha=3$, and different carrier frequencies $f_{c}$. (Fully-loaded cellular system with co-channel interfering cells in two tier, $N_{F I}=18$, and multiple tier, $N_{N I}=330$; extra path loss exponent, $\rho=4$; MS and BS antenna heights : $h_{m}=1.5 \mathrm{~m}$ and $h_{b}=15 \mathrm{~m}$ )

1 Single tier co-channel interfering model $\left(f_{c}=900 \mathrm{MHz}\right)$ [77]

2 Multiple tier co-channel interfering model $\left(f_{c}=900 \mathrm{MHz}\right)$

3 Single tier co-channel interfering model $\left(f_{c}=2 \mathrm{GHz}\right)$ [77]

4 Multiple tier co-channel interfering model $\left(f_{c}=2 \mathrm{GHz}\right)$

5 Single tier co-channel interfering model $\left(f_{c}=3.35 \mathrm{GHz}\right)[77]$

6 Multiple tier co-channel interfering model $\left(f_{c}=3.35 \mathrm{GHz}\right)$

7 Single tier co-channel interfering model $\left(f_{c}=8.45 \mathrm{GHz}\right)[77]$

8 Multiple tier co-channel interfering model $\left(f_{c}=8.45 \mathrm{GHz}\right)$

9 Single tier co-channel interfering model $\left(f_{c}=15.75 \mathrm{GHz}\right)[77]$

10 Multiple tier co-channel interfering model $\left(f_{c}=15.75 \mathrm{GHz}\right)$ 


\subsubsection{Impact of Extra Path Loss Exponent-Multiple Tier Interfer- ence}

Figures $5.31-5.34$, depict the effect of the extra path loss exponent, $\rho$ on the information capacity, when basic path loss exponent $\alpha$ is constant, and equals two. The figures confirm that $A S E$ increases as $\rho$ becomes bigger, which is verified in [77], for the conventional interference model and also holds for the proposed interference model. The $A S E$ tends to increase because $\rho$ affects the far field interferer [77].

The curves in Figure 5.31, show that for $\rho=2$ and $f_{c}=900 \mathrm{MHz}$, the difference in $A S E$ between the two interferers model was $17.09 \%$ and $7.43 \%$, at 0.1 and $0.3 \mathrm{~km}$. For $2 \mathrm{GHz}$, at $R=0.1 \mathrm{~km}$, there was $24.08 \%$ decrease in $A S E$ between the two interferers models. At $R=0.3 \mathrm{~km}$, the decrease was $10.11 \%$. For $f_{c}=3.35 \mathrm{GHz}$, the decrease in information capacity between the two interferers models was $29.39 \%$ and $12.9 \%$, at 0.1 and $0.3 \mathrm{~km}$. For $f_{c}=8.45 \mathrm{GHz}$, the decrease in $A S E$ between the two models was $39.48 \%$ at $0.1 \mathrm{~km}$ and $20.56 \%$ at $0.3 \mathrm{~km}$. For $f_{c}=15.75 \mathrm{GHz}$ the decrease was $45.18 \%$ and $26.96 \%$ at 0.1 and $0.3 \mathrm{~km}$.

The curves in Figure 5.32, show that for $\rho=3$, and $f_{c}=900 \mathrm{MHz}$, the difference in $A S E$ between the two interferers model was $9.60 \%$ and $3.12 \%$ at 0.1 and $0.3 \mathrm{~km}$. For 2 $\mathrm{GHz}$, at $R=0.1 \mathrm{~km}$, there was a $15.58 \%$ decrease in $A S E$ between the two models. At $R=$ $0.3 \mathrm{~km}$, the decrease was $4.75 \%$. For $f_{c}=3.35 \mathrm{GHz}$, the decrease in $A S E$ between the two interferers models was $21.21 \%$ at $0.1 \mathrm{~km}$, and $6.65 \%$ at $0.3 \mathrm{~km}$. For $f_{c}=8.45 \mathrm{GHz}$, the decrease in $A S E$ between the two models was $33.09 \%$ at $0.1 \mathrm{~km}$ and $13.38 \%$ at $0.3 \mathrm{~km}$. For $f_{c}=15.75 \mathrm{GHz}$, at $R=0.1$ and $0.3 \mathrm{~km}$, the decrease was 40.53 and $20.18 \%$.

Figure 5.33, shows that for $\rho=5, f_{c}=900 \mathrm{MHz}$, and $2 \mathrm{GHz}$, there was not much difference in $A S E$ between the two interferers models, as compared to when $f_{c}=3.35,8.45$ and $15.75 \mathrm{GHz}$. For $3.35 \mathrm{GHz}$, the decrease was $11.66 \%$ at $0.1 \mathrm{~km}$. For $8.45 \mathrm{GHz}$, the decrease in $A S E$ between the two interferers models at $0.1 \mathrm{~km}$, was $23.63 \%$, and $6.00 \%$ at $0.3 \mathrm{~km}$. For $f_{c}=15.75 \mathrm{GHz}$, at $R=0.1$ and $0.3 \mathrm{~km}$, the decrease was 32.77 and $11.71 \%$.

Figure 5.34, shows that for $\rho=8$ and $f_{c}=900 \mathrm{MHz}, 2 \mathrm{GHz}$ and $3.35 \mathrm{GHz}$, there was not much difference in $A S E$ between the single and multiple tier interferers model. However, at $8.45 \mathrm{GHz}$, there was a decrease of $14.86 \%$ at $R=0.1 \mathrm{~km}$, and $2.19 \%$ at $R=$ 
$0.3 \mathrm{~km}$. For $f_{c}=15.75 \mathrm{GHz}$, at $R=0.1$ and $0.3 \mathrm{~km}$, the decrease was $24.34 \%$ and $5.61 \%$. An important conclusion derived from the curves in Figure 5.31 - 5.34, is that at higher carrier frequencies and smaller extra path loss exponent, other tiers of co-channel interfering cells apart from those in the first tier become active. Other tiers of co-channel interfering cells become active because as carrier frequency increases the breakpoint distance of the two-slope path loss model increases (refer to Figure 5.5). The increase in breakpoint distance allows other co-channel interfering cells to be in the same region (basic path loss exponent, $(\alpha)$ region), as those of the first tier. Likewise, as the extra path loss exponent $\rho$ increases the decrease in $A S E$ between the two interferers model turns to decrease. The $A S E$ tend to decrease, because interference after the breakpoint becomes less severe, even in the case of higher carrier frequency and smaller cell size radius. 


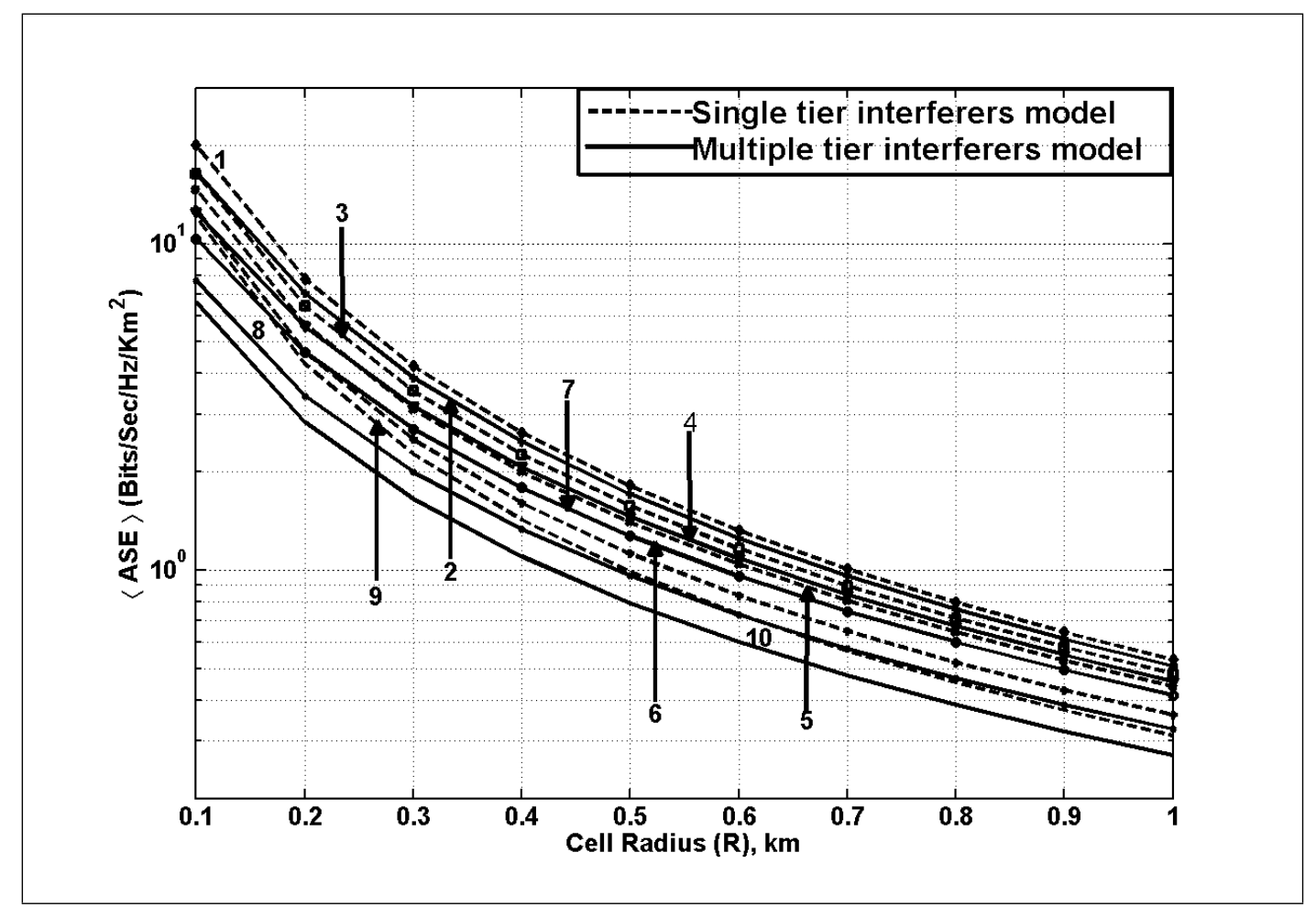

Figure 5.31: Average uplink area spectral efficiency versus cell radius for extra path loss exponent, $\rho=2$, and different carrier frequencies, $f_{c}$. (Fully-loaded cellular system with co-channel interfering cells in first tier, $N_{F I}=6$, and multiple tier, $N_{N I}=330 ;$; path loss exponent, $\alpha=2$; MS and BS antenna heights : $h_{m}=1.5 \mathrm{~m}$ and $h_{b}=15 \mathrm{~m}$ )

1 Single tier co-channel interfering model $\left(f_{c}=900 \mathrm{MHz}\right)[77]$

2 Multiple tier co-channel interfering model $\left(f_{c}=900 \mathrm{MHz}\right)$

3 Single tier co-channel interfering model $\left(f_{c}=2 \mathrm{GHz}\right)[77]$

4 Multiple tier co-channel interfering model $\left(f_{c}=2 \mathrm{GHz}\right)$

5 Single tier co-channel interfering model $\left(f_{c}=3.35 \mathrm{GHz}\right)$ [77]

6 Multiple tier co-channel interfering model $\left(f_{c}=3.35 \mathrm{GHz}\right)$

7 Single tier co-channel interfering model $\left(f_{c}=8.45 \mathrm{GHz}\right)$ [77]

8 Multiple tier co-channel interfering model $\left(f_{c}=8.45 \mathrm{GHz}\right)$

9 Single tier co-channel interfering model $\left(f_{c}=15.75 \mathrm{GHz}\right)$ [77]

10 Multiple tier co-channel interfering model $\left(f_{c}=15.75 \mathrm{GHz}\right)$ 


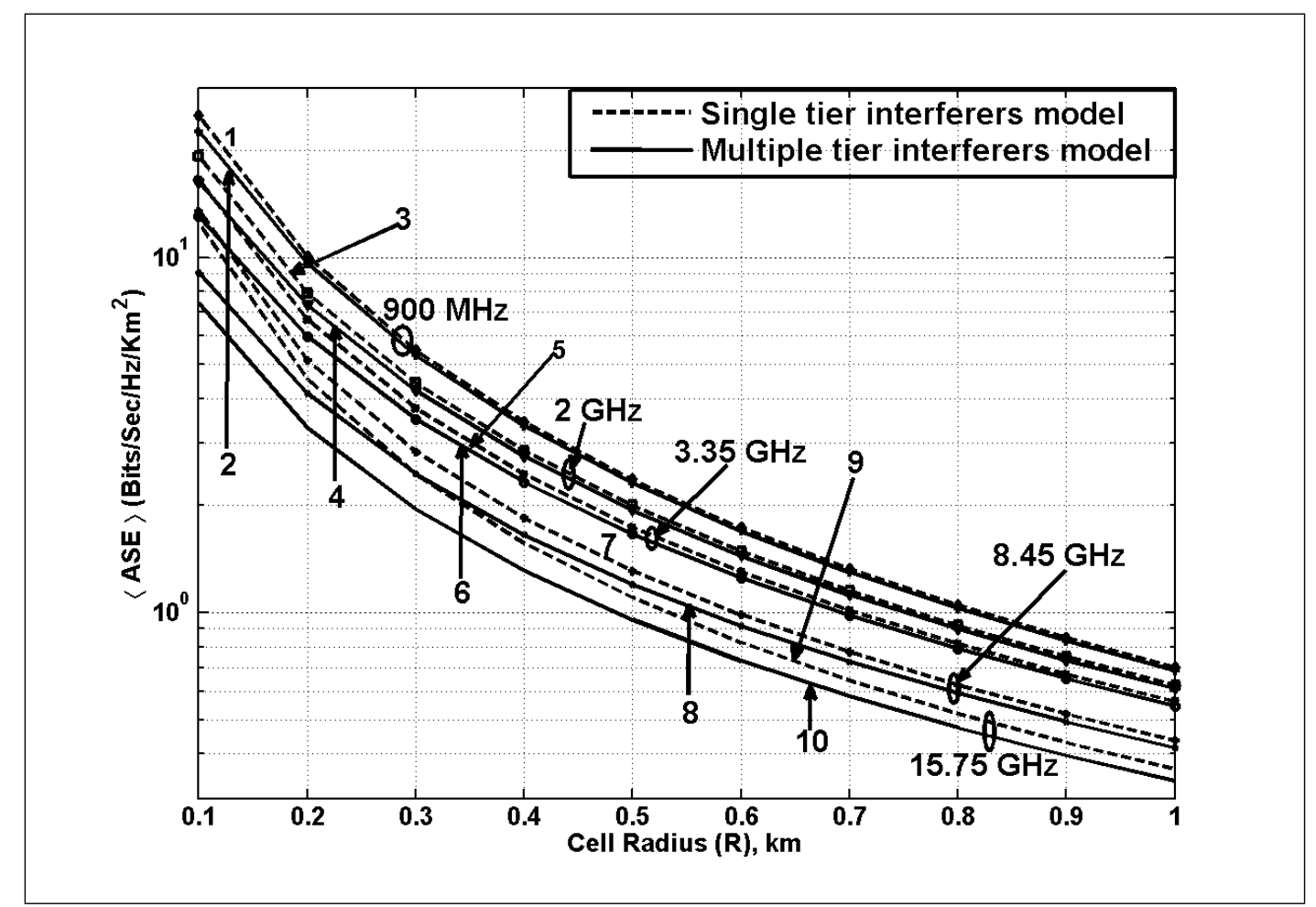

Figure 5.32: Average uplink area spectral efficiency versus cell radius for extra path loss exponent, $\rho=3$, and different carrier frequencies, $f_{c}$. (Fully-loaded cellular system with co-channel interfering cells in first tier, $N_{F I}=6$, and multiple tier, $N_{N I}=330 ;$; path loss exponent, $\alpha=2$; MS and BS antenna heights : $h_{m}=1.5 \mathrm{~m}$ and $h_{b}=15 \mathrm{~m}$ )

1 Single tier co-channel interfering model $\left(f_{c}=900 \mathrm{MHz}\right)$ [77]

2 Multiple tier co-channel interfering model $\left(f_{c}=900 \mathrm{MHz}\right)$

3 Single tier co-channel interfering model $\left(f_{c}=2 \mathrm{GHz}\right)[77]$

4 Multiple tier co-channel interfering model $\left(f_{c}=2 \mathrm{GHz}\right)$

5 Single tier co-channel interfering model $\left(f_{c}=3.35 \mathrm{GHz}\right)$ [77]

6 Multiple tier co-channel interfering model $\left(f_{c}=3.35 \mathrm{GHz}\right)$

7 Single tier co-channel interfering model $\left(f_{c}=8.45 \mathrm{GHz}\right)$ [77]

8 Multiple tier co-channel interfering model $\left(f_{c}=8.45 \mathrm{GHz}\right)$

9 Single tier co-channel interfering model $\left(f_{c}=15.75 \mathrm{GHz}\right)$ [77]

10 Multiple tier co-channel interfering model $\left(f_{c}=15.75 \mathrm{GHz}\right)$ 


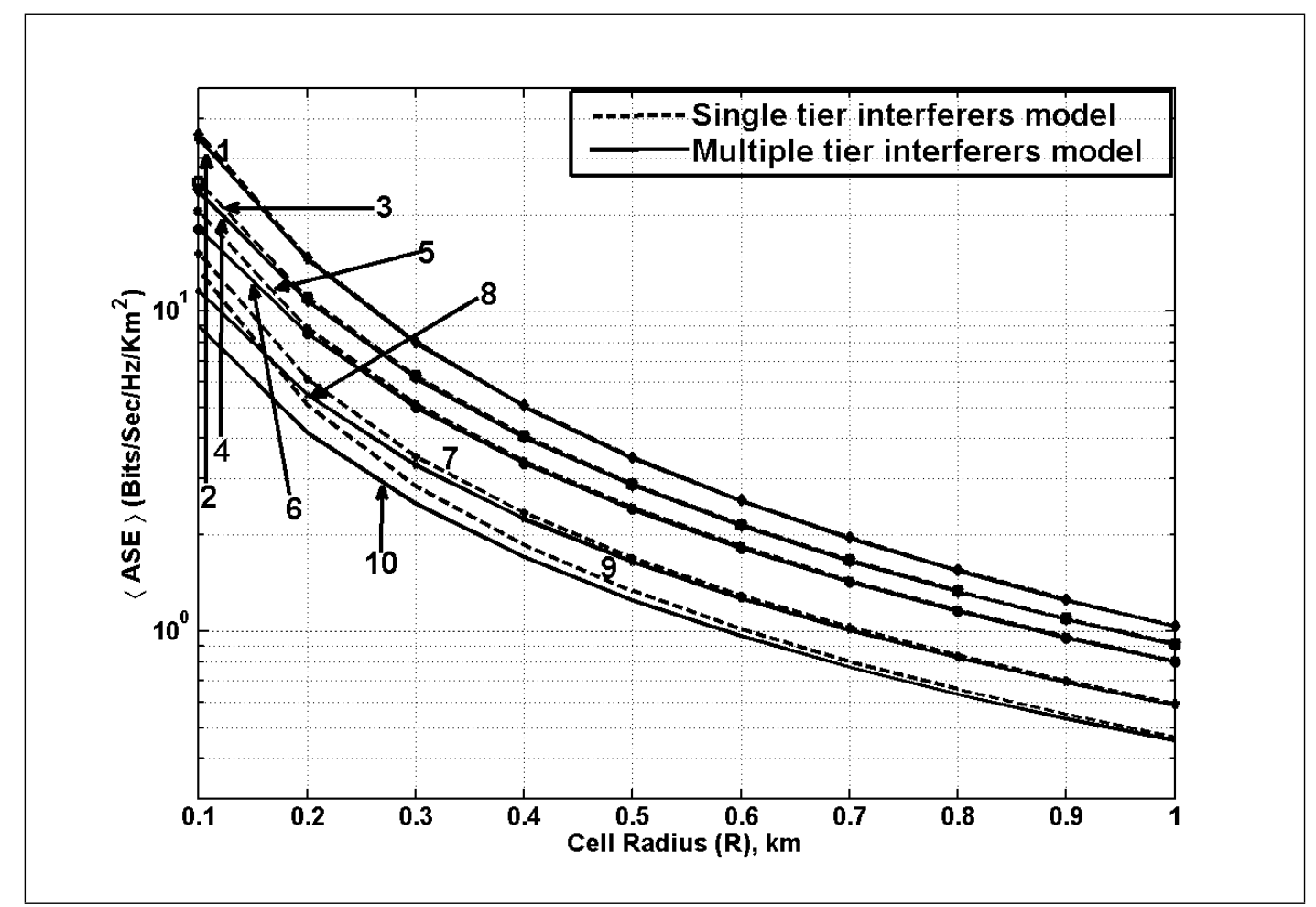

Figure 5.33: Average uplink area spectral efficiency versus cell radius for extra path loss exponent, $\rho=5$, and different carrier frequencies, $f_{c}$. (Fully-loaded cellular system with co-channel interfering cells in first tier, $N_{F I}=6$, and multiple tier, $N_{N I}=330 ;$; path loss exponent, $\alpha=2$; MS and BS antenna heights : $h_{m}=1.5 \mathrm{~m}$ and $h_{b}=15 \mathrm{~m}$ )

1 Single tier co-channel interfering model $\left(f_{c}=900 \mathrm{MHz}\right)[77]$

2 Multiple tier co-channel interfering model $\left(f_{c}=900 \mathrm{MHz}\right)$

3 Single tier co-channel interfering model $\left(f_{c}=2 \mathrm{GHz}\right)[77]$

4 Multiple tier co-channel interfering model $\left(f_{c}=2 \mathrm{GHz}\right)$

5 Single tier co-channel interfering model $\left(f_{c}=3.35 \mathrm{GHz}\right)$ [77]

6 Multiple tier co-channel interfering model $\left(f_{c}=3.35 \mathrm{GHz}\right)$

7 Single tier co-channel interfering model $\left(f_{c}=8.45 \mathrm{GHz}\right)$ [77]

8 Multiple tier co-channel interfering model $\left(f_{c}=8.45 \mathrm{GHz}\right)$

9 Single tier co-channel interfering model $\left(f_{c}=15.75 \mathrm{GHz}\right)$ [77]

10 Multiple tier co-channel interfering model $\left(f_{c}=15.75 \mathrm{GHz}\right)$ 


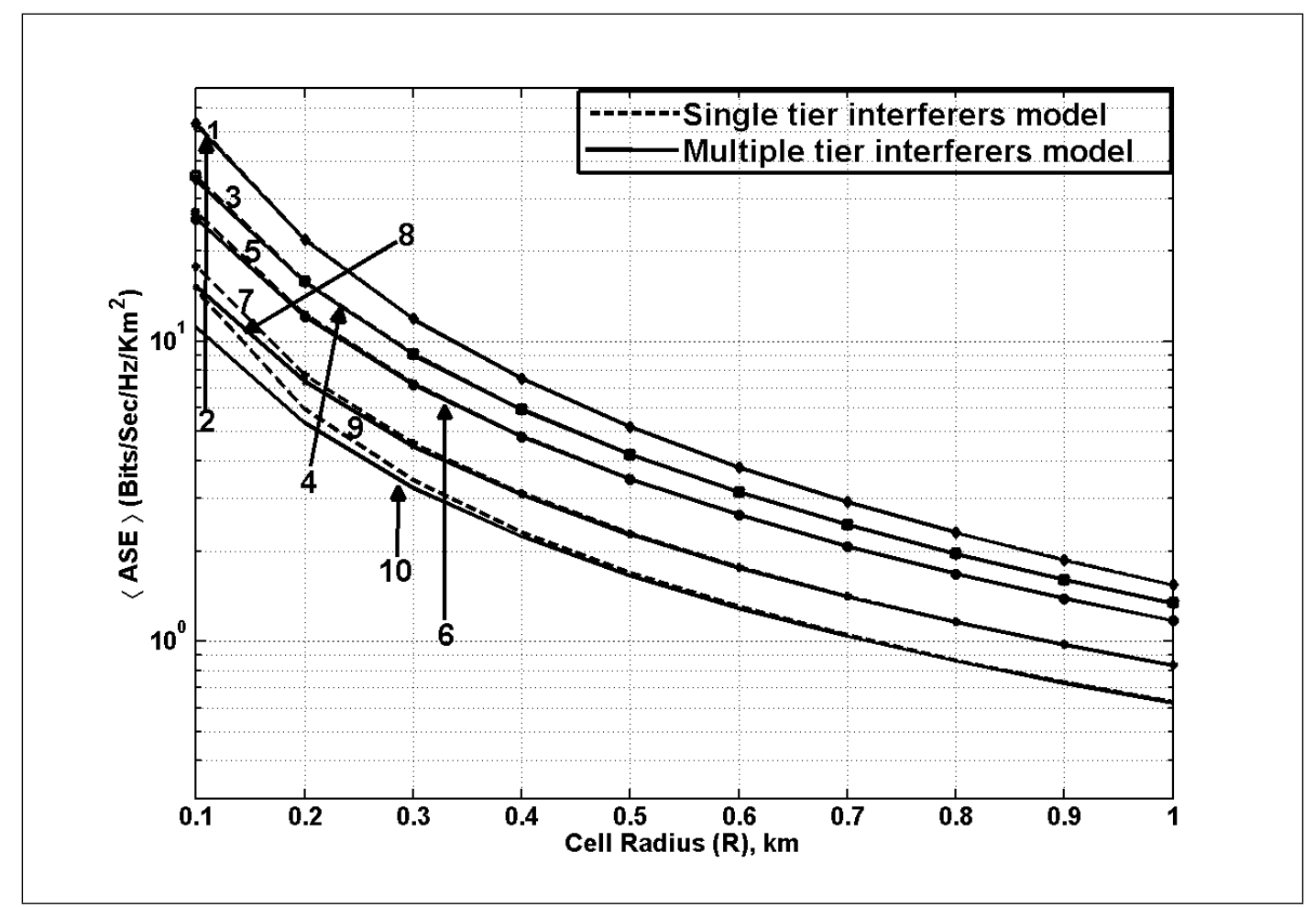

Figure 5.34: Average uplink area spectral efficiency versus cell radius for extra path loss exponent, $\rho=8$, and different carrier frequencies, $f_{c}$. (Fully-loaded cellular system with co-channel interfering cells in first tier, $N_{F I}=6$, and multiple tier, $N_{N I}=330 ;$; path loss exponent, $\alpha=2$; MS and BS antenna heights : $h_{m}=1.5 \mathrm{~m}$ and $h_{b}=15 \mathrm{~m}$ )

1 Single tier co-channel interfering model $\left(f_{c}=900 \mathrm{MHz}\right)$ [77]

2 Multiple tier co-channel interfering model $\left(f_{c}=900 \mathrm{MHz}\right)$

3 Single tier co-channel interfering model $\left(f_{c}=2 \mathrm{GHz}\right)[77]$

4 Multiple tier co-channel interfering model $\left(f_{c}=2 \mathrm{GHz}\right)$

5 Single tier co-channel interfering model $\left(f_{c}=3.35 \mathrm{GHz}\right)$ [77]

6 Multiple tier co-channel interfering model $\left(f_{c}=3.35 \mathrm{GHz}\right)$

7 Single tier co-channel interfering model $\left(f_{c}=8.45 \mathrm{GHz}\right)$ [77]

8 Multiple tier co-channel interfering model $\left(f_{c}=8.45 \mathrm{GHz}\right)$

9 Single tier co-channel interfering model $\left(f_{c}=15.75 \mathrm{GHz}\right)$ [77]

10 Multiple tier co-channel interfering model $\left(f_{c}=15.75 \mathrm{GHz}\right)$ 


\subsubsection{Impact of Antenna Height-Multiple Tier Interference}

Figures 5.31, 5.35 and 5.36, show the plot of information capacity versus cell radius. The plot is for different BS antenna heights, when $\alpha=2, \rho=2$, and $f_{c}=0.9,2,3.35,8.45$ and $15.75 \mathrm{GHz}$. The plots are for studying the impact of BS antenna height on a cellular system operating at a higher carrier frequency and having a smaller cell size radius. For Figure 5.31, the decrease in information capacity between the two interference models has been given in Chapter 5.7.2. For base station antenna height $\mathrm{h}_{b}=35 \mathrm{~m}$, Figure 5.35, shows that at $900 \mathrm{MHz}$, the decreases at 0.1 and $0.3 \mathrm{~km}$, were $24.37 \%$ and $10.34 \%$. For $2 \mathrm{GHz}$, when $R=0.1$ and $0.3 \mathrm{~km}$, the curves show a decrease in $A S E$ of $33.06 \%$ and $15.28 \%$. At $R=0.1$ and $0.3 \mathrm{~km}$, for $3.35 \mathrm{GHz}$, the decrease was $38.71 \%$ and $19.81 \%$. For $8.45 \mathrm{GHz}$, at $R=0.1$ and $0.3 \mathrm{~km}$, the decrease in ASE was $46.89 \%$ and $29.31 \%$. Finally for 15.75 $\mathrm{GHz}$, the decrease was $50.53 \%$ and $35.38 \%$ at 0.1 and $0.3 \mathrm{~km}$.

Fig. 5.36, compares the information capacity for the two interference models, when $\mathrm{h}_{b}=55 \mathrm{~m}$. For $f_{c}=900 \mathrm{MHz}$, the curves show that at $R=0.1$ and $0.3 \mathrm{~km}$, the decrease in $A S E$ was $29.16 \%$ and $12.82 \%$. At $f_{c}=2 \mathrm{GHz}$, the curves show that at $R=0.1$ and $0.5 \mathrm{~km}$, the decrease in $A S E$ was $38.02 \%$ and $19.20 \%$, likewise for $f_{c}=3.35 \mathrm{GHz}$, at $R=0.1$ and $0.3 \mathrm{~km}$ the decrease in $A S E$ was 43.07 and $24.35 \%$. For $8.45 \mathrm{GHz}$, the curves show that at $R=0.1 \mathrm{~km}$, the decrease in $A S E$ was $49.66 \%$, and at $0.3 \mathrm{~km}$, it was $33.81 \%$. Finally for $15.75 \mathrm{GHz}$, the decrease was $52.31 \%$ and $38.98 \%$ at 0.1 and $0.3 \mathrm{~km}$.

By examination of Figures 5.31, 5.35 and 5.36, we can concluded that at carrier frequencies greater than $2 \mathrm{GHz}$ as the BS antenna height increases co-channel interference from other tiers becomes more severe. This leads to a much decrease in $A S E$. The reason being that as the BS antenna height increases more co-channel interfering cells are in the region before the breakpoint, where power fall off is $\propto 1 /(\text { distance })^{2}$. 


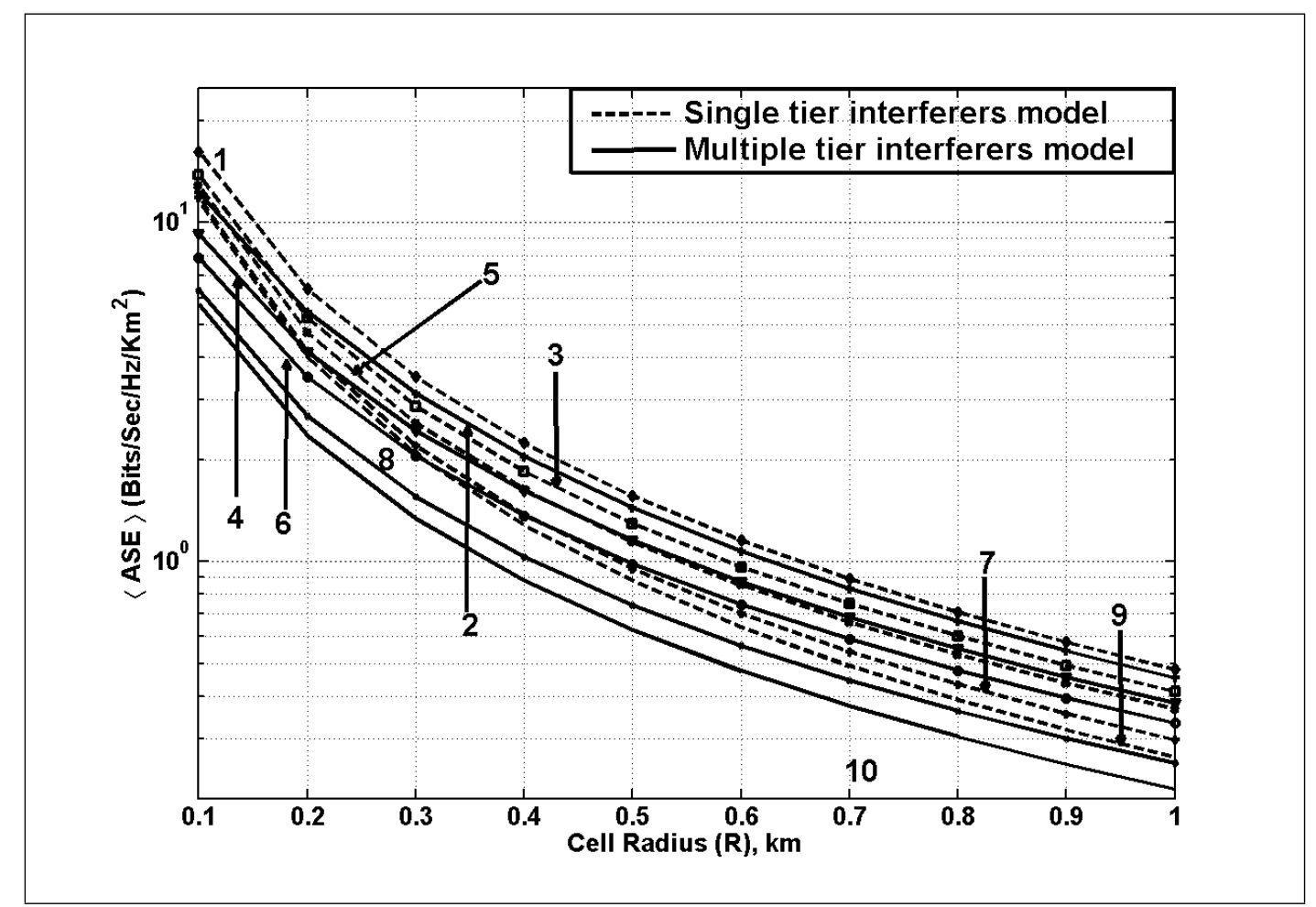

Figure 5.35: Average uplink area spectral efficiency versus cell radius for BS antenna height $h_{b}=35 \mathrm{~m}$, and different carrier frequencies, $f_{c}$. (Fully-loaded cellular system with co-channel interfering cells in first tier, $N_{F I}=6$, and multiple tier, $N_{N I}=330$; path loss exponent, $\alpha=2$, extra path loss exponent, $\rho=2$; MS antenna heights : $h_{m}=1.5 \mathrm{~m}$ )

1 Single tier co-channel interfering model $\left(f_{c}=900 \mathrm{MHz}\right)$ [77]

2 Multiple tier co-channel interfering model $\left(f_{c}=900 \mathrm{MHz}\right)$

3 Single tier co-channel interfering model $\left(f_{c}=2 \mathrm{GHz}\right)[77]$

4 Multiple tier co-channel interfering model $\left(f_{c}=2 \mathrm{GHz}\right)$

5 Single tier co-channel interfering model $\left(f_{c}=3.35 \mathrm{GHz}\right)$ [77]

6 Multiple tier co-channel interfering model $\left(f_{c}=3.35 \mathrm{GHz}\right)$

7 Single tier co-channel interfering model $\left(f_{c}=8.45 \mathrm{GHz}\right)$ [77]

8 Multiple tier co-channel interfering model $\left(f_{c}=8.45 \mathrm{GHz}\right)$

9 Single tier co-channel interfering model $\left(f_{c}=15.75 \mathrm{GHz}\right)$ [77]

10 Multiple tier co-channel interfering model $\left(f_{c}=15.75 \mathrm{GHz}\right)$ 


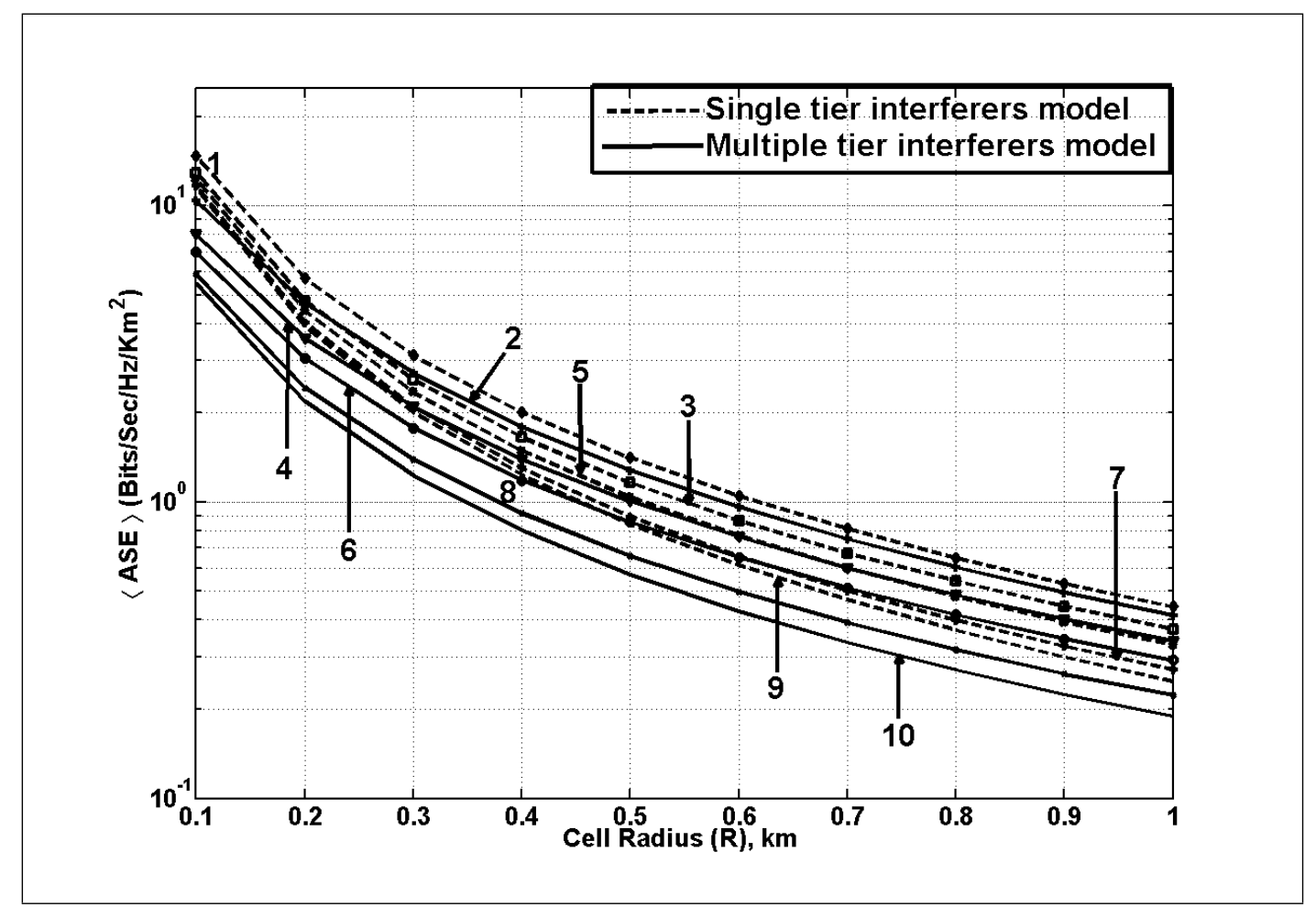

Figure 5.36: Average uplink area spectral efficiency versus cell radius for BS antenna height $h_{b}=55 \mathrm{~m}$, and different carrier frequencies, $f_{c}$. (Fully-loaded cellular system with co-channel interfering cells in first tier, $N_{F I}=6$, and multiple tier, $N_{N I}=330$; path loss exponent, $\alpha=2$, extra path loss exponent, $\rho=2$; MS antenna heights : $h_{m}=1.5 \mathrm{~m}$ )

1 Single tier co-channel interfering model $\left(f_{c}=900 \mathrm{MHz}\right)$ [77]

2 Multiple tier co-channel interfering model $\left(f_{c}=900 \mathrm{MHz}\right)$

3 Single tier co-channel interfering model $\left(f_{c}=2 \mathrm{GHz}\right)[77]$

4 Multiple tier co-channel interfering model $\left(f_{c}=2 \mathrm{GHz}\right)$

5 Single tier co-channel interfering model $\left(f_{c}=3.35 \mathrm{GHz}\right)$ [77]

6 Multiple tier co-channel interfering model $\left(f_{c}=3.35 \mathrm{GHz}\right)$

7 Single tier co-channel interfering model $\left(f_{c}=8.45 \mathrm{GHz}\right)$ [77]

8 Multiple tier co-channel interfering model $\left(f_{c}=8.45 \mathrm{GHz}\right)$

9 Single tier co-channel interfering model $\left(f_{c}=15.75 \mathrm{GHz}\right)$ [77]

10 Multiple tier co-channel interfering model $\left(f_{c}=15.75 \mathrm{GHz}\right)$ 


\subsubsection{Impact of Vehicular Traffic - Multiple Tier Interference}

Figures 5.37 - 5.40, show the simulation result for the impact of vehicular traffic, (effective road height, $h$ ) on the information capacity of a cellular wireless system operating at higher carrier frequencies. The curves for light vehicular traffic, and BS antenna $h_{b}=15 \mathrm{~m}$, are shown in Figure 5.37. The curves show that for $f_{c}=3.35,8.45$ and $15.75 \mathrm{GHz}$, at $R=100$ $\mathrm{m}$, the decrease in the information capacity between the two interference model was 29.18 , 39.29 and $45.01 \%$. At $R=300 \mathrm{~m}$, the decrease was 12.80, 20.36 and $26.75 \%$. Figure 5.38, shows the case for heavy vehicular traffic, and $h_{b}=15 \mathrm{~m}$. At $100 \mathrm{~m}$, the decrease in $A S E$ was $18.18,26.65$ and $33.60 \%$. At $300 \mathrm{~m}$, the percentage decrease in $A S E$ between the two interference models was $7.79,11.47,15.672 \%$.

The numerical simulation result in Figure 5.39, shows the curve for light vehicular traffic, and BS antenna height $h_{b}=35 \mathrm{~m}$. For carrier frequencies $f_{c}=3.35,8.45$ and 15.75 $\mathrm{GHz}$, at $100 \mathrm{~m}$, cell size radius the decrease in $A S E$ was 38.63, 46.83, 50.50\%. For $300 \mathrm{~m}$, it was $19.73,29.22$ and $35.27 \%$. Finally Figure 5.40, shows the curve for heavy vehicular traffic, and $h_{b}=55 \mathrm{~m}$. For carrier frequencies $f_{c}=3.35,8.45$ and $15.75 \mathrm{GHz}$, at $100 \mathrm{~m}$, cell size radius the decrease in $A S E$ was $26.47,36.66,42.90 \%$. For 300 m, it was 11.34 , 18.01 and $24.17 \%$.

The numerical simulation result shows that even for different vehicular traffic conditions as the carrier frequency increases, and cell size radius decreases multiple tiers of co-channel interference become active. This, therefore, leads to interference becoming severe, thus decreasing the information capacity of the cellular wireless communication system. The curves also show that for both light and heavy vehicular traffic conditions as the BS antenna height increases, co-channel interference becomes more severe. It becomes severe because as the BS antenna height increases the breakpoint distance increases and more co-channel cells are in the free space region of the path loss model.

From Figures $5.37-5.40$, it is evident that the information capacity performance for both co-channel interference models improves for heavy vehicular traffic as compared to that of light vehicular traffic, which is in agreement with the work in [28].

The figures also reveal that the decrease in ASE between the two interference models is less, when vehicular traffic is heavy as compared to light vehicular traffic. From 


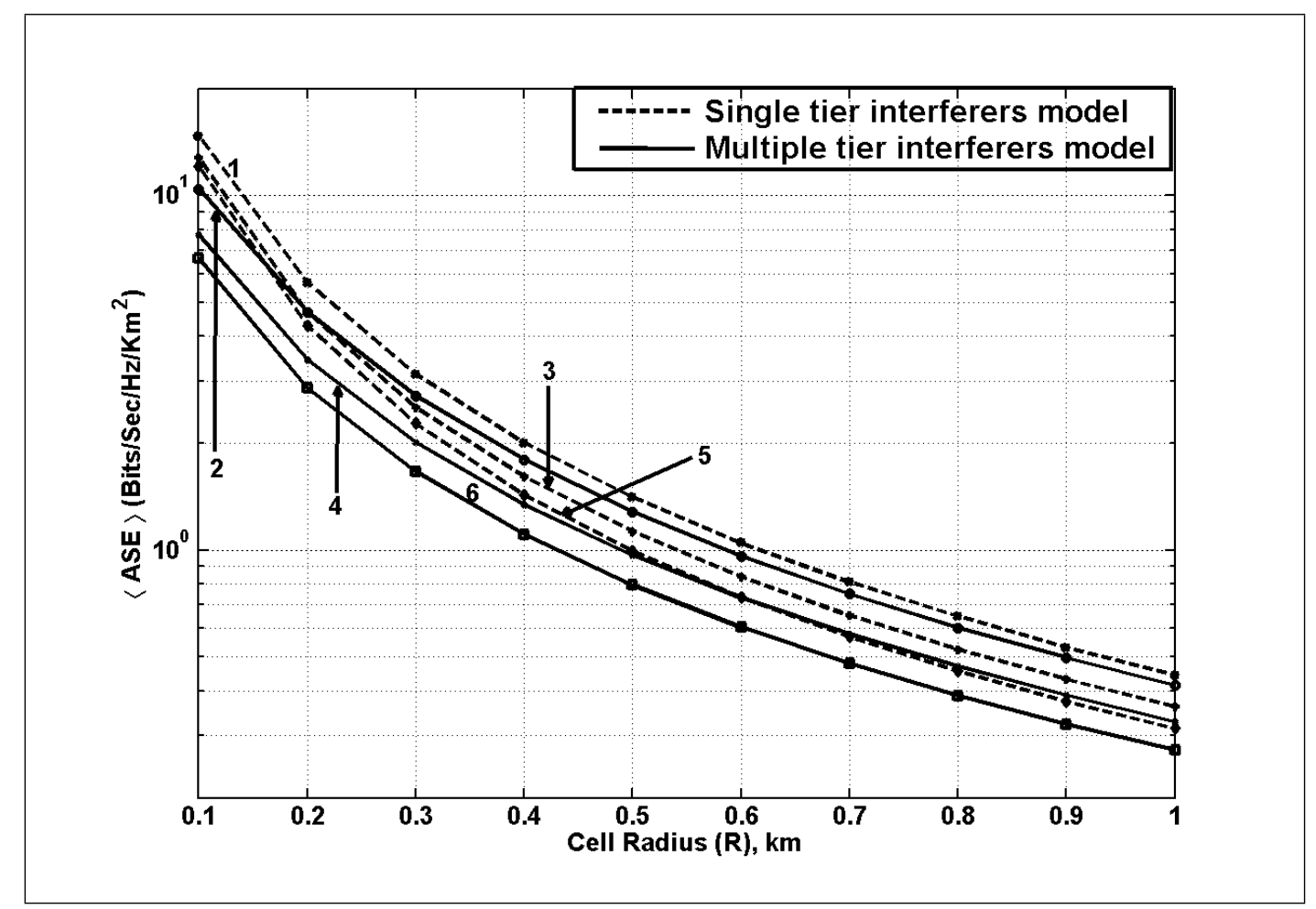

Figure 5.37: Average uplink area spectral efficiency versus cell radius for heavy vehicular traffic with effective road height, $h=0.3 \mathrm{~m}$ and BS antenna height, $h_{b}=15 \mathrm{~m}$ for different carrier frequencies, $f_{c}$. (Fully-loaded cellular system with co-channel interfering cells $N_{F I}=6$ in the first tier, and $N_{N I}=330$, multiple tier co-channel interferers; and path loss exponent, $\alpha=2$ and extra path loss exponent $\rho=2$; MS antenna height, $h_{m}=1.8 \mathrm{~m}$ )

1 Single tier co-channel interfering model $\left(f_{c}=3.35 \mathrm{GHz}\right)$

2 Multiple tier co-channel interfering model $\left(f_{c}=3.35 \mathrm{GHz}\right)$

3 Single tier co-channel interfering model $\left(f_{c}=8.45 \mathrm{GHz}\right)$

4 Multiple tier co-channel interfering model $\left(f_{c}=8.45 \mathrm{GHz}\right)$

5 Single tier co-channel interfering model $\left(f_{c}=15.75 \mathrm{GHz}\right)$

6 Multiple tier co-channel interfering model $\left(f_{c}=15.75 \mathrm{GHz}\right)$ 


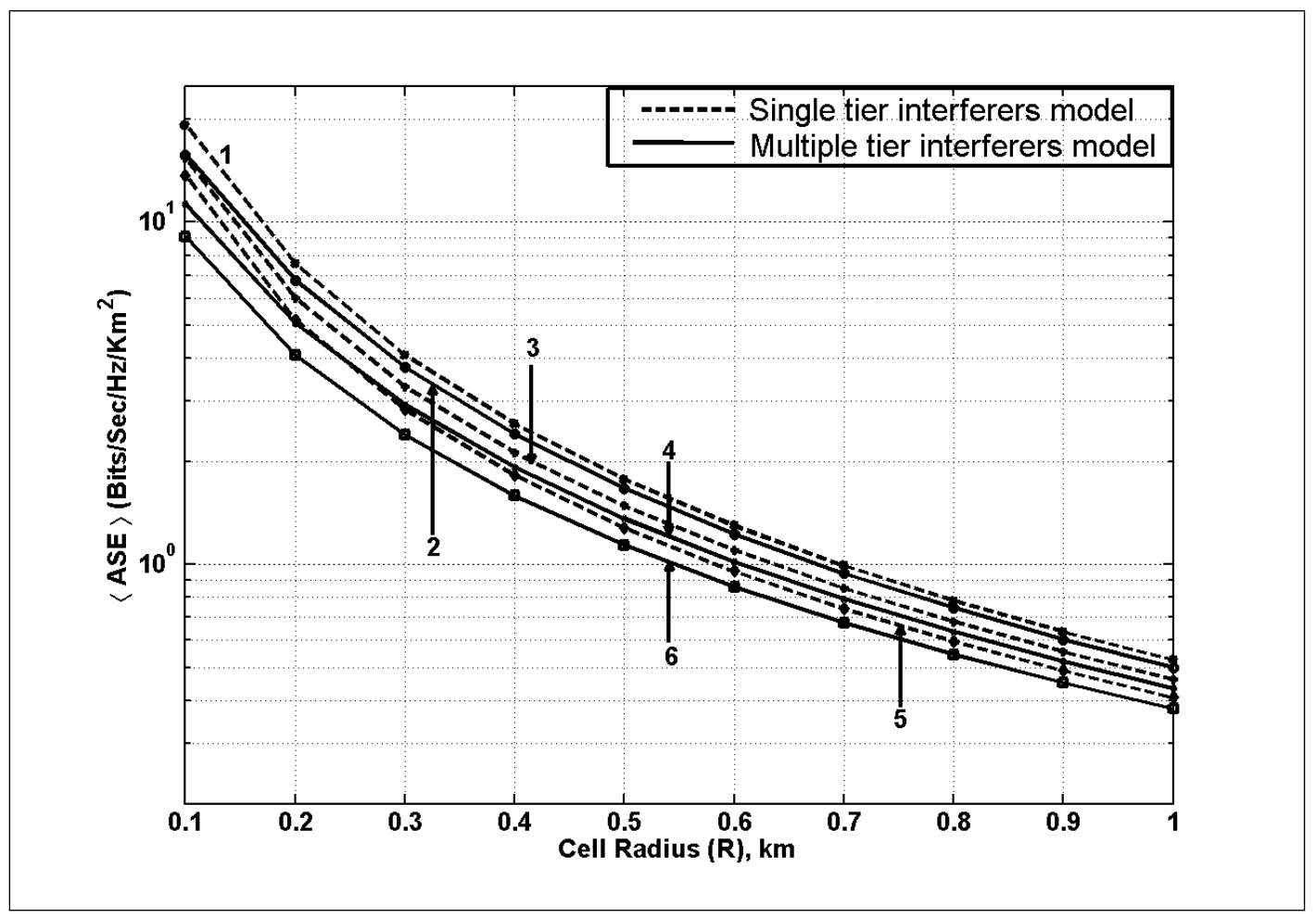

Figure 5.38: Average uplink area spectral efficiency versus cell radius for heavy vehicular traffic with effective road height, $h=1.29 \mathrm{~m}$ and BS antenna height, $h_{b}=15 \mathrm{~m}$ for different carrier frequencies, $f_{c}$. (Fully-loaded cellular system with co-channel interfering cells $N_{F I}=6$ in the first tier, and $N_{N I}=330$, multiple tier co-channel interferers; and path loss exponent, $\alpha=2$ and extra path loss exponent $\rho=2$; MS antenna height, $h_{m}=1.8 \mathrm{~m}$ )

1 Single tier co-channel interfering model $\left(f_{c}=3.35 \mathrm{GHz}\right)$

2 Multiple tier co-channel interfering model $\left(f_{c}=3.35 \mathrm{GHz}\right)$

3 Single tier co-channel interfering model $\left(f_{c}=8.45 \mathrm{GHz}\right)$

4 Multiple tier co-channel interfering model $\left(f_{c}=8.45 \mathrm{GHz}\right)$

5 Single tier co-channel interfering model $\left(f_{c}=15.75 \mathrm{GHz}\right)$

6 Multiple tier co-channel interfering model $\left(f_{c}=15.75 \mathrm{GHz}\right)$ 


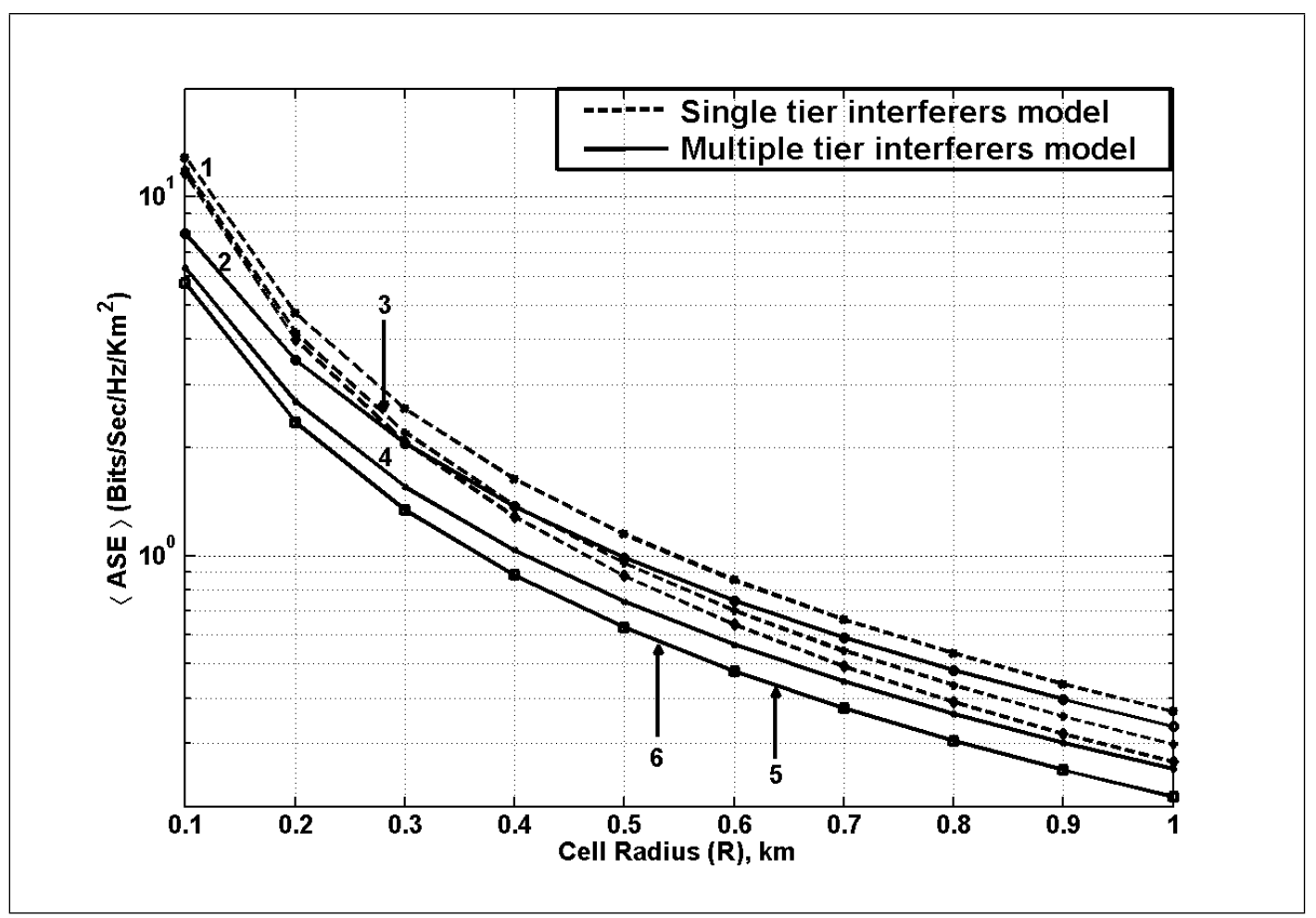

Figure 5.39: Average uplink area spectral efficiency versus cell radius for heavy vehicular traffic with effective road height, $h=0.3 \mathrm{~m}$ and BS antenna height, $h_{b}=35 \mathrm{~m}$ for different carrier frequencies, $f_{c}$. (Fully-loaded cellular system with co-channel interfering cells $\mathrm{N}_{F I}=6$ in the first tier, and $N_{N I}=330$, multiple tier co-channel interferers; and path loss exponent, $\alpha=2$ and extra path loss exponent $\rho=2$; MS antenna height, $\mathrm{h}_{m}=1.8 \mathrm{~m}$ )

1 Single tier co-channel interfering model $\left(f_{c}=3.35 \mathrm{GHz}\right)$

2 Two tier co-channel interfering model $\left(f_{c}=3.35 \mathrm{GHz}\right)$

3 Single tier co-channel interfering model $\left(f_{c}=8.45 \mathrm{GHz}\right)$

4 Two tier co-channel interfering model $\left(f_{c}=8.45 \mathrm{GHz}\right)$

5 Single tier co-channel interfering model $\left(f_{c}=15.75 \mathrm{GHz}\right)$

6 Two tier co-channel interfering model $\left(f_{c}=15.75 \mathrm{GHz}\right)$ 


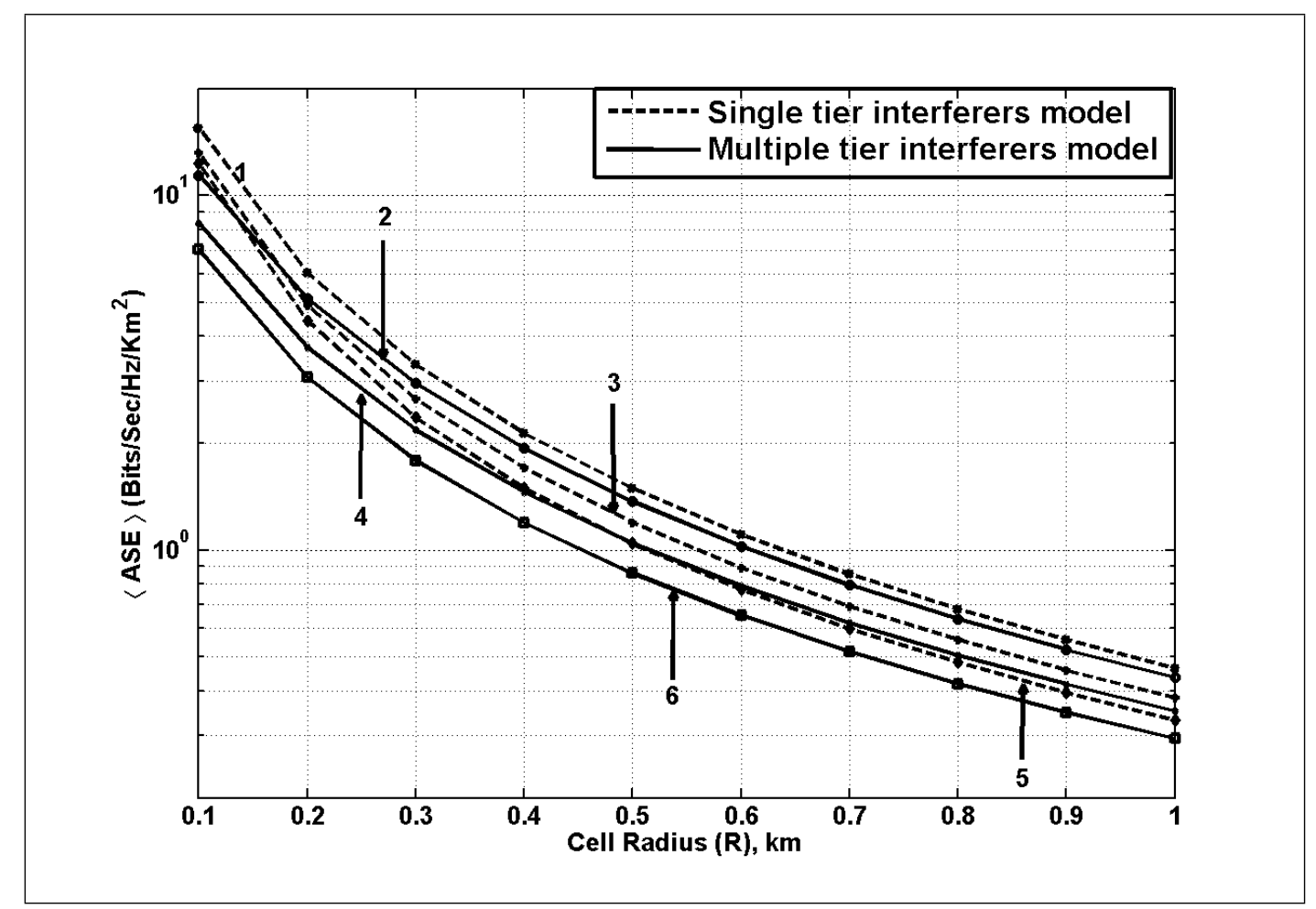

Figure 5.40: Average uplink area spectral efficiency versus cell radius for heavy vehicular traffic with effective road height, $h=1.29 \mathrm{~m}$ and BS antenna height, $h_{b}=35 \mathrm{~m}$ for different carrier frequencies, $f_{c}$. (Fully-loaded cellular system with co-channel interfering cells $N_{F I}=6$ in the first tier, and $N_{N I}=330$, multiple tier co-channel interferers; and path loss exponent, $\alpha=2$ and extra path loss exponent $\rho=2$; MS antenna height, $h_{m}=1.8 \mathrm{~m}$ )

1 Single tier co-channel interfering model $\left(f_{c}=3.35 \mathrm{GHz}\right)$

2 Two tier co-channel interfering model $\left(f_{c}=3.35 \mathrm{GHz}\right)$

3 Single tier co-channel interfering model $\left(f_{c}=8.45 \mathrm{GHz}\right)$

4 Two tier co-channel interfering model $\left(f_{c}=8.45 \mathrm{GHz}\right)$

5 Single tier co-channel interfering model $\left(f_{c}=15.75 \mathrm{GHz}\right)$

6 Two tier co-channel interfering model $\left(f_{c}=15.75 \mathrm{GHz}\right)$ 
the result we can conclude that communication during the day when traffic is heavy takes advantage of the favorable propagation conditions to improve information capacity.

\subsection{Summary}

In this chapter, because of the dependence of cellular wireless communication system information capacity on propagation loss and system parameters we have analyzed, and studied the impact of both system and propagation loss parameters on the information capacity performance of a TDMA based LOS microcellular wireless network. The wireless network is operating at carrier frequencies greater than $2 \mathrm{GHz}$, and multiple tier of cochannel interferers are considered to be active. Our performance analysis was based on the uplink information capacity, the two-slope propagation loss model, and the characterization of $A S E$ as a function of cell size radius.

Based on our interference model, coupled with mathematical analysis, and simulation. It was found that at microwave carrier frequencies greater than $2 \mathrm{GHz}$ as, (i) BS antenna height increases (ii) cell size radius reduces (iii) basic path loss exponent decreases, and (iv) extra path loss exponent decreases; multiple tiers of co-channel interfering cells become active. They become active because the breakpoint location is far from the transmitting source. This makes other tiers of co-channel interfering cells to be in the same region as that of the first tier, which is the free-space region of the two-slope path loss graph (that is before the break-point). Unlike carrier frequencies less than $2 \mathrm{GHz}$, where the breakpoint is closer to the transmitting source, hence only the first tier co-channel interfering cells are in the free-space region of the two-slope path loss graph. The other tier co-channel interfering cells are in the region after the breakpoint, which makes them negligible. Interference becomes negligible, because after the breakpoint the received signal power decreases in inverse proportion to the fourth power of distance. Our analysis and simulation supports the claim that as cell size radius reduces, other tier co-channel interference becomes active. The analysis shows that the information capacity of a cellular wireless system depends on the frequency of operation, which is related to the breakpoint distance.

The result from this chapter also shows that the uplink information capacity of 
cellular wireless networks is sensitive to propagation/system parameters. Hence it is imperative to have a proper characterization of the propagation conditions to plan accurately and design a cellular network. In addition, it is shown that for heavy vehicular traffic the effect of other tier co-channel interfering cells on the uplink information capacity of the cellular wireless network is less as compare to light vehicular traffic. We therefore, conclude that communication during the day, when traffic is heavy, takes advantage of the favorable propagation conditions to increase the uplink information capacity of a cellular wireless communication system. 


\section{Chapter 6}

\section{Minimum Cell Size for Information}

Capacity Increase in Cellular Wireless

Network 


\subsection{Introduction}

The main goal, of this thesis is to find a minimum cell size for information capacity increase in cellular wireless communication systems. The minimum cell size is found for a cellular network operating at higher carrier frequencies, greater than $2 \mathrm{GHz}$ and smaller cell size radius less than $1000 \mathrm{~m}$. This is a very challenging problem due to the fact that it is difficult to find the exact theoretical limit.

In the previous chapters, we have proposed a two tier and a multiple tier co-channel interference model. Using the conventional and proposed co-channel interference model, we provided information capacity performance analysis on a cellular wireless communication system operating at higher carrier frequencies and smaller cell size radius. We have shown, that in cellular wireless communication systems operating at higher microwave carrier frequencies greater than $2 \mathrm{GHz}$, and smaller cell size radius, multiple tiers of cochannel interference become active.

In the past wireless communication systems were required to accommodate a large number of voice and/or low-speed data services. Now, as stated in earlier chapters of this thesis emerging wireless communication systems require large system capacity. They require large capacity, because of the increase of mobile subscribers, and the introduction of multimedia services by network providers, which require high speed data-rate [2]. Therefore there is a need to utilize the limited radio spectrum available for wireless communication systems efficiently, because it is regulated by governments, and international agreements $[26,27]$.

Cellular systems have partly been used to achieve high spectrum efficiency by exploiting the power falloff with distance of signal propagation to reuse the same frequency channel at a spatially separated location [4]. Other techniques such as, (i) combination of bandwidth efficient coding/modulation techniques at the communication link level (ii) the use of sophisticated channel allocation schemes that minimize the overall carried traffic at the network or system levels, have also been employed to to increase the spectral efficiency [31]. However, a high overall spectrum efficiency is achieved at the frequency planning level by cell size radius reduction $[37,38]$. The growth in information capacity of wireless communication systems have led to cell sites being installed in ever-increasing 
densities, leading to cell size radius becoming smaller [16]. In conventional cellular wireless communication system the assumption of increase in information capacity by cell size radius reduction was based on a large path loss exponent [7].

Higher carrier frequencies are also employed to increase the data rate in a wireless network [28]. However, as the carrier frequency increases both the free space path loss and diffraction loss increases according to increase in frequency [2]. The increase in path and diffraction loss leads to a reduction in cell size radius [2]. Cell size radius reduction in the center of Tokyo led to an increase in co-channel interference [1]. The decrease in cell size radius causes co-channel interference to become severe and difficult to control [2]. Though, smaller cell size radius increases the information capacity of a cellular wireless system, other-cell interference lower it [40]. Therefore, for cellular wireless systems regardless of the cell size radius, co-channel interference is a limiting factor for information capacity increase $[38,120]$. Reducing cell size radius in a cellular wireless system, means more cells are required, which is always costly [41].

In general, because of co-channel interference cell size radius cannot be reduced indefinitely to achieve higher information capacity [121]. Therefore, there is a limit to cell size reduction, which is in agreement with the suggestion by Zhou et al. in [7]. In previous research works ranges for maximum and minimum cell size radius and the fact that shrinking cell size increase information capacity of cellular system have being reported [4-7]. However, quantitatively there is no theoretical limit to cell size reduction. The majority of the research work on cell size radius reduction for information capacity increase was based on technical gains, such as spectral efficiency, neglecting economic efficiency, such as cost. The economic efficiency is as important as the technical gains, because as cell size radius reduces for the information capacity to be increased, the cost of the cellular network also increases.

In this chapter, using the proposed multiple tier interference model from the previous chapters, mathematical analysis, supported by computer simulation, is used to find a theoretical limit to cell size reduction in cellular wireless networks. Capacity - cost, based on the uplink information capacity of TDMA based cellular wireless communication system, is used in finding the theoretical limit. Our main contribution in this chapter is as follows: 
- Information capacity - cost performance analysis is used to find a theoretical limit to cell size radius reduction in cellular wireless network.

The reminder of this chapter is organized as follows. The system model is presented in Chapter 6.2. Chapter 6.3, presents the cost estimation model. In Chapter 6.4, we present the analysis of information capacity/cost, for the cellular wireless communication system. The setup for numerical simulation is presented in Chapter 6.5. Numerical results from the simulation are then presented in Chapter 6.6, followed by a summary in Chapter 6.10. The work presented in this chapter is published in $[45,51]$.

\subsection{System Model for Finding Minimum Cell Size Radius}

We consider a cellular wireless communication system operating at higher carrier frequen$\operatorname{cies}\left(f_{c}>2 \mathrm{GHz}\right)$, and having smaller cell size radius, $(100 \mathrm{~m} \leq R \leq 1000 \mathrm{~m})$. Co-channel interference is from multiple tiers. A two-dimensional hexagonal non-sectorized, smaller cell size radius network as described in Chapter 5.3.2, and depicted in Figure 5.7 is considered. All cells are assumed to have omnidirectional antennas. Cell $\mathrm{BS}_{0}$, we refer to as the reference cell and there are n-tiers of co-channel interfering cells. Now a cell $j$ is served by $\mathrm{BS}_{j}$, which is located at the cell's center, with $1 \leq \mathrm{j} \leq 6$, for first tier co-channel interferes, $7 \leq \mathrm{j} \leq 18$, for second tier co-channel interferes, and $[((n-1) \times 6)+1] \leq \mathrm{j} \leq$ $[((\mathrm{n}-1) \times 6)+6 \mathrm{n}]$, for the nth tier co-channel interferes. $D$ (refer to Figure. 5.7), is the distance between $\mathrm{BS}_{0}$ and a first tier BS. A second tier $\mathrm{BS}$ is therefore, at a distance $2 \mathrm{D}$ from $\mathrm{BS}_{0}$. An nth tier $\mathrm{BS}$ is located at distance $\mathrm{n} D$. Each cell site has a single user.

\subsubsection{Multiple Tier Co-channel Interference Model}

The co-channel interference model is described in Chapter 5.3.2, but for ease of reading we repeat the mathematical description. Let $r[\mathrm{~m}]$, denote the distance from the desired MS to desired BS. $g$ the breakpoint distance from the transmitting source. Let $\mathrm{n}$ denote the nth tier, $R_{u}$ the reuse distance. $R_{i n}$ is the nth interfering cell size radius. The multiple 
tier desired MS's carrier-to-interference ratio is mathematical represented as

$$
\gamma_{d}\left(N_{n}, r\right)=\frac{r^{-\alpha}(1+r / g)^{-\rho}}{\sum_{i=1}^{\infty} \sum_{n=1}^{6 N_{n}}\left(n r_{i n}\right)^{-\alpha}\left(1+n r_{i n} / g\right)^{-\rho}} .
$$

where $\alpha$ and $\rho$ denotes the basic and extra path loss exponent.

\subsection{Cost Estimation System Model}

The cost of a cellular wireless communication system is a multi-disciplinary subject that extends to economic and business aspects. They extends as well to various technical disciplines, such as network planning, radio resource management and physical layer performance. Therefore, there is a need to simplify the model used in this work. We considered one technology strategy for the wireless communication system, where one technology is used as far as possible even for service where it is not optimal.

In practice there is a very large number of possible configurations of base stations, including different alternatives for sites, transmission, etc. In the cost analysis, we consider an omni-directional smaller cell size radius cellular base station, with antennas below rooftop. In the following section, we describe the general infrastructure cost model for a smaller cell size radius cellular wireless communication system. The key assumptions made in the infrastructure cost analysis in our system model are as follows:

\subsubsection{Infrastructure Cost}

We limit the infrastructure cost to the radio access network as shown in Figure 6.1. Core networks, signalling protocols, service delivery platforms, marketing and administration costs are explicitly excluded. They are excluded because of their low cost in comparison to radio access infrastructure cost. We also excluded the cost of radio spectrum as it is to a large extent driven by aspects that are difficult to model and predict; aspects such as the entrance of a new operator and political environment.

To quantify the cost of the cellular wireless network, we adopt the infrastructure cost model in which the total infrastructure cost is modelled linearly proportional to the 


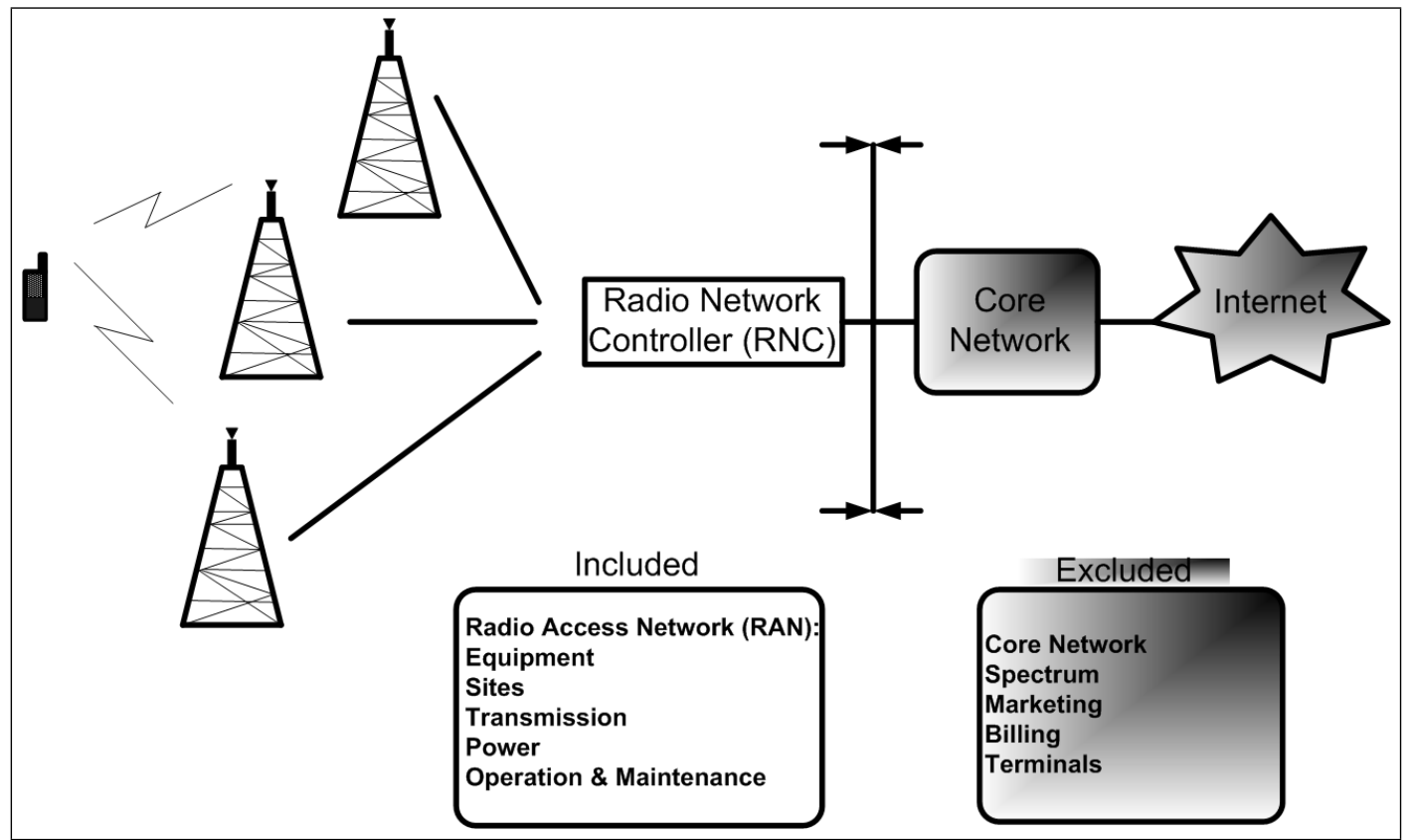

Figure 6.1: Infrastructure cost model for finding minimum cell size radius.

number of BS and is given by [122].

$$
T_{\mathcal{C}}=B S_{\mathcal{C}} N_{B S}
$$

where $N_{B S}$ is the number of BSs'. $B S_{\mathcal{C}}$ is a constant, which corresponds to the cost per BS. We assumed $B S_{\mathcal{C}}$ to be the same for all BS and it is independent of the BS characteristics, which is not the case in practical system. In a practical system a number of different BS types can be used for different deployment scenarios. As a consequence the cost structure of a radio access network is dependent on the system configuration. For the cost structure we took into account both the capital expenditure (CAPEX) and operation expenditure (OPEX). The cost drivers with respect to CAPEX and OPEX, for the radio access network are summarized in Table 6.1. In the infrastructure cost model discount rate and transmission prices yearly erosion are neglected. Base station CAPEX is estimated to be 150000 per unit cost ( $£, \$$, etc), and OPEX to be 75000 per unit cost ( $£, \$$, etc).

Now the total cost per $\mathrm{BS} B S_{\mathcal{C}}$ is given by

$$
B S_{\mathcal{C}}=C A P E X+O P E X
$$


Information Capacity/Cost Performance Analysis

\begin{tabular}{|l|l|}
\hline CAPEX & OPEX \\
\hline Base station equipment & Electric power \\
\hline Base station (site) installation & Operation and maintenance \\
\hline Site buildout $(\rho)$ & Site lease \\
\hline Backhaul transmission equipment & Backhaul transmission lease \\
\hline Radio network controller equipment & \\
\hline
\end{tabular}

Table 6.1: Basic cost drivers for radio access network

where CAPEX is the capital expenditure, and OPEX is the operation expenditure. Note that the network being considered can either be coverage or capacity limited. The number of BS required per square kilometer is dimensioned by

$$
N_{B S}=\frac{1}{\pi R^{2}}
$$

where $R$ is the cell radius. We also assume a continuous service area. From equation (6.2), (6.3) and (6.4), the total infrastructure cost $T_{\mathcal{C}}$ is expressed as a function of cell size radius $R$, and it is written as

$$
T_{\mathcal{C}}=\frac{1}{\pi R^{2}}(C A P E X+O P E X)
$$

The unit cost per $\mathrm{km}^{2}$, of a cellular wireless communication system, as a function of cell size radius, using the linear cost model in equation (6.5), is illustrated in Figure 6.2. The linear cost model shows, that as cell size radius reduces the unit cost per $\mathrm{km}^{2}$ increases in proportional to $1 / R^{2}$.

\subsection{Information Capacity/Cost Performance Analysis}

The information capacity model described in Chapter 6.2, for multiple tier co-channel interference, and the cost model presented in Chapter 6.3, are used to formulate a mutual information capacity - cell size radius - cost relationship. The relationship is used to find the minimum cell size radius for information capacity increase in a cellular wireless communication system.

The formulated relationship is given by the ratio of $A S E$ for multiple tier co-channel 


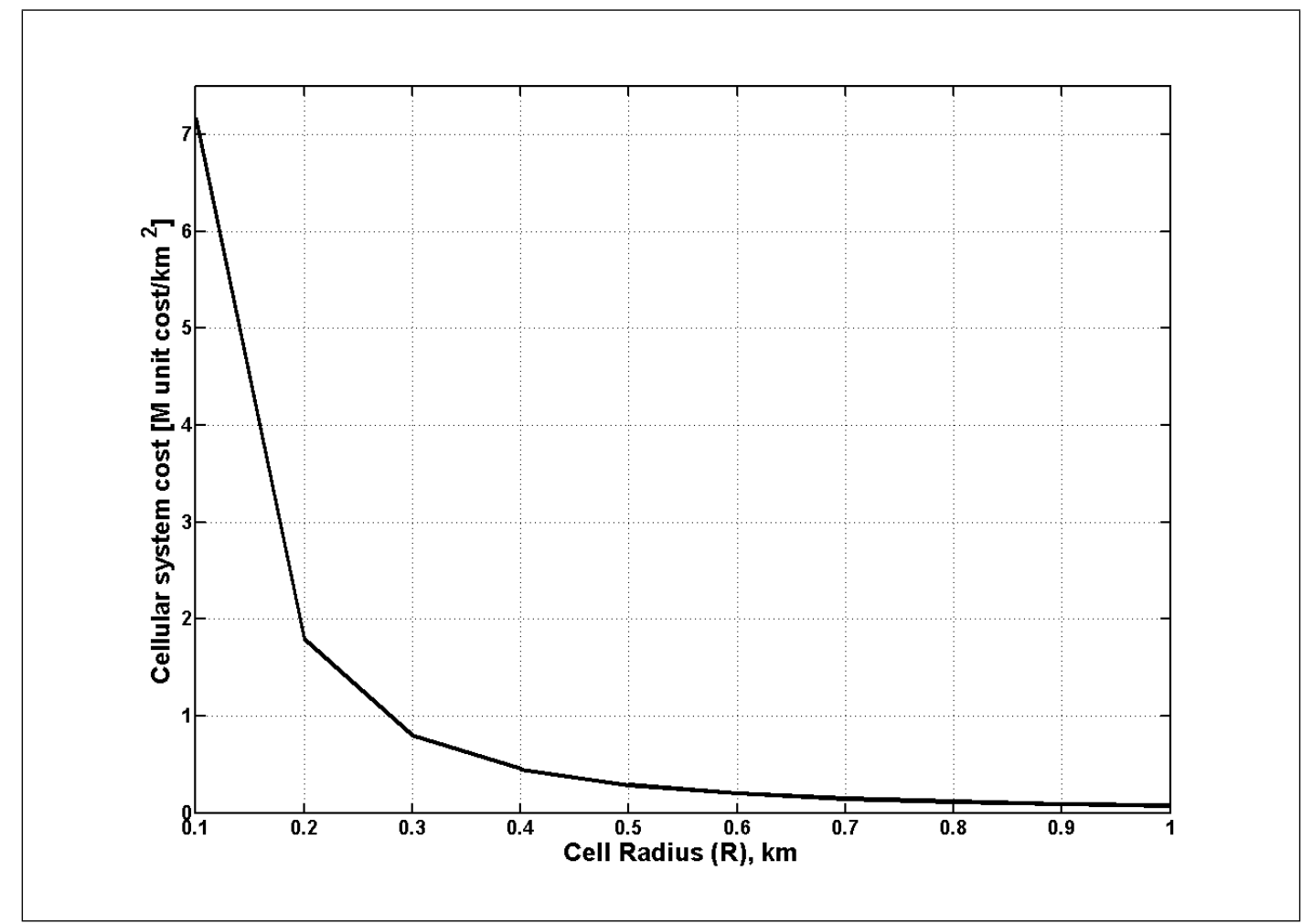

Figure 6.2: Cellular system unit cost per $\mathrm{km}^{2}$ versus cell radius $\mathrm{R}$.

interference to the total infrastructure cost $T_{\mathcal{C}}$. Therefore, the ratio of $A S E$ and $T_{\mathcal{C}}, A_{\text {Ratio }}$ is written as

$$
A_{\text {Ratio }}=\frac{\left\langle A_{e}\left(r, N_{J}\right)\right\rangle}{T_{\mathcal{C}}}=\frac{\frac{4}{\pi R_{u}^{2} R^{2}} \int_{R_{0}}^{R} \log _{2}(1+\gamma) p_{\gamma}(r) d r}{\frac{1}{\pi R^{2}}(C A P E X+O P E X)}
$$

where $R$ is the cell size radius. $R_{u}$ is the normalized reuse distance, and $R_{0}$ corresponds to the closest distance a MS can be from a BS antenna. $\gamma$ represents the desired MS carrierto-interference ratio, CIR. The distribution of the user's CIR is described by the probability density function (PDF) $\mathrm{p}_{\gamma}(r)$ as in subsection 4.2.1.

Simplifying equation (6.6), we re-write $A_{\text {Ratio }}$ as follows

$$
A_{\text {Ratio }}=\frac{4}{R_{u}^{2}(C A P E X+O P E X)} \int_{R_{0}}^{R} \log _{2}(1+\gamma) p_{\gamma}(r) d r
$$

Note that $A_{\text {Ratio }}$, given in equation (6.7), is for omni-directional small cell size radius deployment, and interference limited cellular wireless communication environment. 


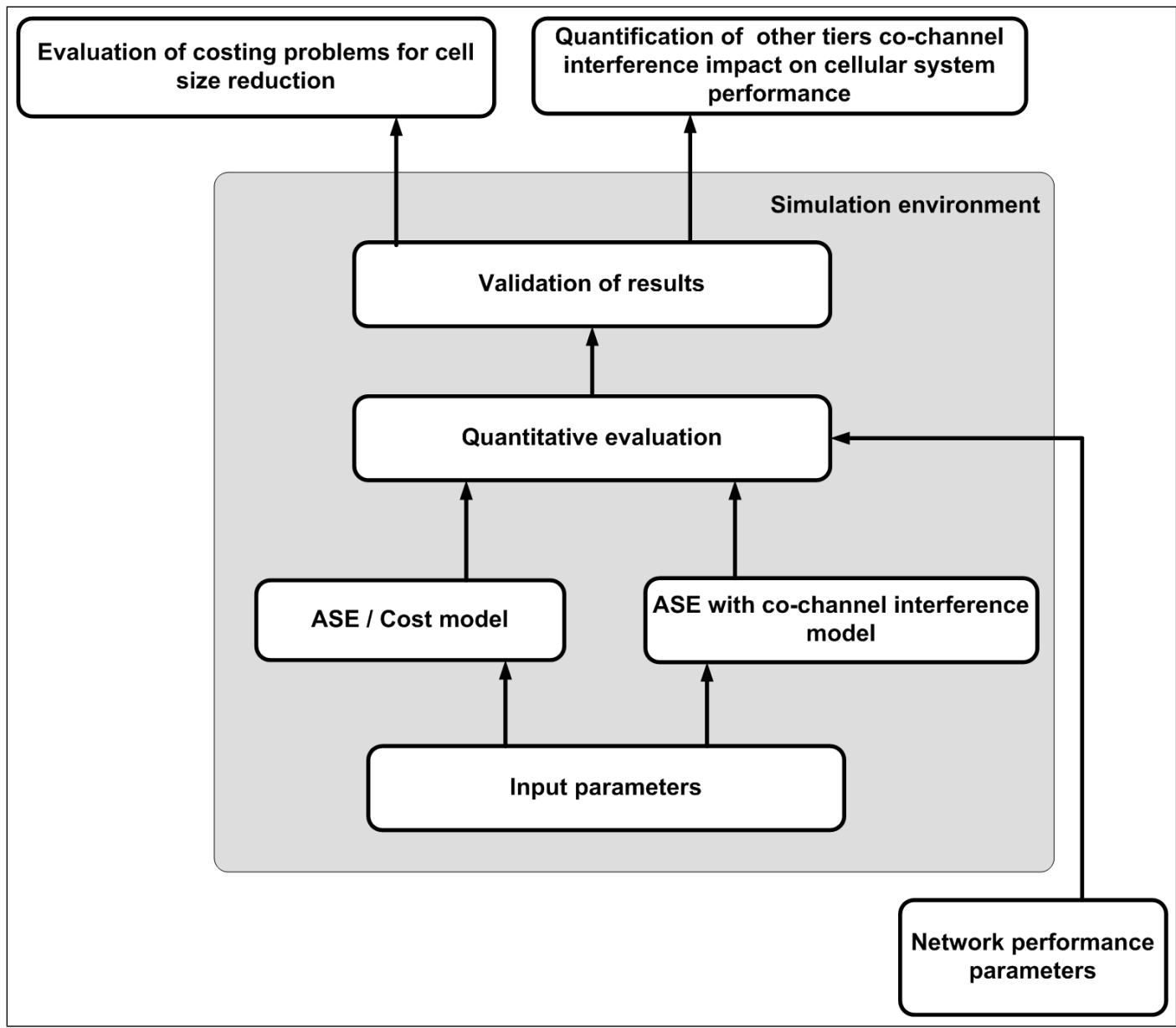

Figure 6.3: Integrated simulation environment for information capacity/cost. 


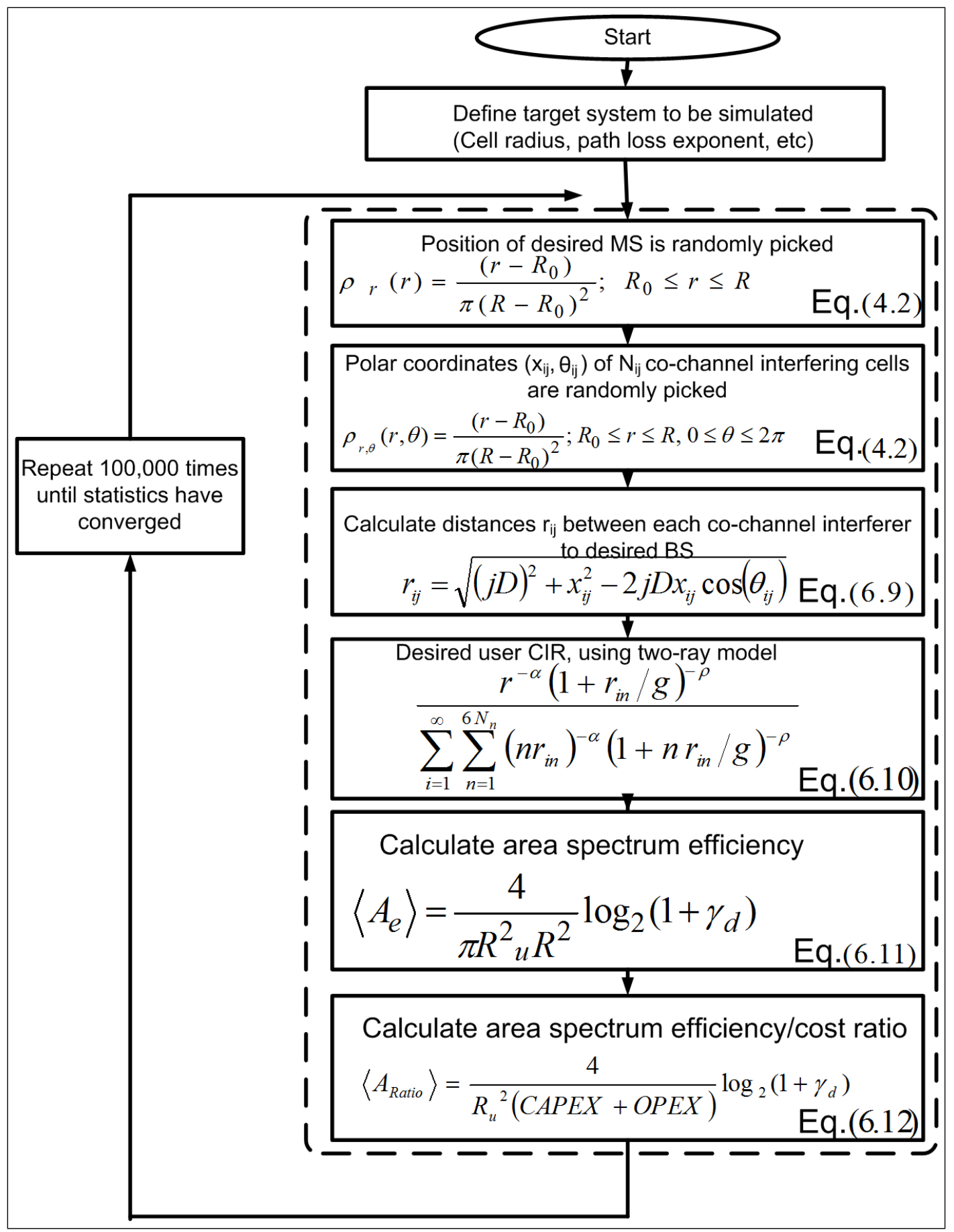

Figure 6.4: Flow chart for information capacity/cost simulations. 


\subsection{Numerical Simulations for Information Capacity/Cost}

From equation (6.7), because MS are randomly located $\left\langle A_{\text {Ratio }}\right\rangle$, is mathematically intractable to explicitly solve analytically. Therefore, we used Monte Carlo simulation to estimate it. Figure 6.3, illustrates the integrated simulation environment for the information capacity/cost model.

\subsubsection{Numerical Simulations Setup}

The simulation result are aimed at finding a minimum cell size radius, for information capacity increase in cellular wireless communication systems when multiple tier of cochannel interference are active. The simulation setup follows the setup described in Chapter 5.6. Figure 6.3 shows an integrated simulation environment for information capacity/cost analysis, and Figure 6.4 shows a flow chart for the simulation setup. In the simulation the desired and interfering users' positions are assumed to be uniformly distributed as described in Chapter 4.2.1 by equation (4.1). Now one step is added the algorithm described in Chapter 5.6 as follows to incorporate the total infrastructure $\operatorname{cost} T_{\mathcal{C}}$, to calculate the $A S E$ to cost ratio $A_{\text {Ratio }}$. The $A S E$ and $C_{\text {ost }}$ total ratio for the cellular wireless system is then calculated by

$$
\left\langle A_{\text {Ratio }}\right\rangle=\frac{4}{R_{u}^{2}(C A P E X+O P E X)} \log _{2}\left(1+\gamma_{d}\right) .
$$

We then repeat the procedure 100000 times, after locating the desired user position. The values of $\left\langle A_{e}\right\rangle$ and $\left\langle A_{\text {Ratio }}\right\rangle$, were then estimated by taking the average of all the observations of and $A_{\text {Ratio }}$, as given by equation (6.8). To ensure consistences we have used the same system parameters as used in previous simulations. Note that we considered only ten tiers of co-channel interfering cells, as successive numerical runs with an increasing number of tiers beyond ten had no effect on the accuracy of our simulation results.

\subsection{Numerical Results}

In this section, we present simulation results, for the performance comparison, of the information capacity/cost ratio $\left\langle A_{\text {Ratio }}\right\rangle$. The information capacity/cost performance compari- 
Numerical Results

son is between cellular systems using our proposed interference model, and those using the conventional interference model. Our information capacity results are an extension of the analysis by [77]. In other to facilitate the understanding of the information capacity/cost ratio $\left\langle A_{\text {Ratio }}\right\rangle$, a review of the information capacity performance for TDMA cellular wireless communication systems operating at higher carrier frequencies and smaller cell size radius in Chapter 5.5 is necessary.

\subsubsection{Information Capacity/Cost Ratio Numerical Results}

In Figures 6.5 - 6.9, we present curves of the ratio of area spectral efficiency $A S E$ to total infrastructure cost $T_{\mathcal{C}}, A_{\text {Ratio }}$ versus cell size radius $R$ at a desired MS, for the conventional and proposed interference model. These figures show that the information capacity per unit cost $(£, \$$, etc), for a given cellular wireless network, tends to increase as the cell size radius reduces. The reason for this is that as cell radius decreases more cells (BS) are required in the network.

Figure 6.5 , shows that for $f_{c}=900 \mathrm{MHz}$, the multiple tier interference model incurs a loss of between $4.20-9.65 \%$, (that is cell radius $1-0.1 \mathrm{~km}$ ), in information capacity per unit cost $(£, \$$, etc), as compared to the single tier interference model. This decrease in information capacity per unit cost, is due to interference from other tiers of co-channel cells.

For $f_{c}=2 \mathrm{GHz}$, Figure 6.6, shows that multiple tier co-channel interference, causes a loss of between $4.93-14.61 \%$, in the information capacity per unit cost ( $£, \$$, etc) as compared to the single tier. When $f_{c}=3.35 \mathrm{GHz}$, Figure 6.7 , the information capacity per unit cost $(£, \$$, etc), loss incurred between the two interference models is between 5.76 - $18.89 \%$. For $f_{c}=8.45 \mathrm{GHz}$, Figure 6.8, shows that the loss incurred in information capacity per unit cost $(£, \$$, etc) between the two interference models is between 8.56 $27.41 \%$. Finally for $f_{c}=15.75 \mathrm{GHz}$, Figure 6.9 the decrease in information capacity per unit cost $(£, \$$, etc) between the two interference models is between $11.76-32.52 \%$.

The results show that, when the carrier frequency increases the loss incurred in information capacity per unit cost $(£, \$$, etc), between the two interference models also increases. As an example, for carrier frequency $f_{c}=900 \mathrm{MHz}$, at cell size radius 


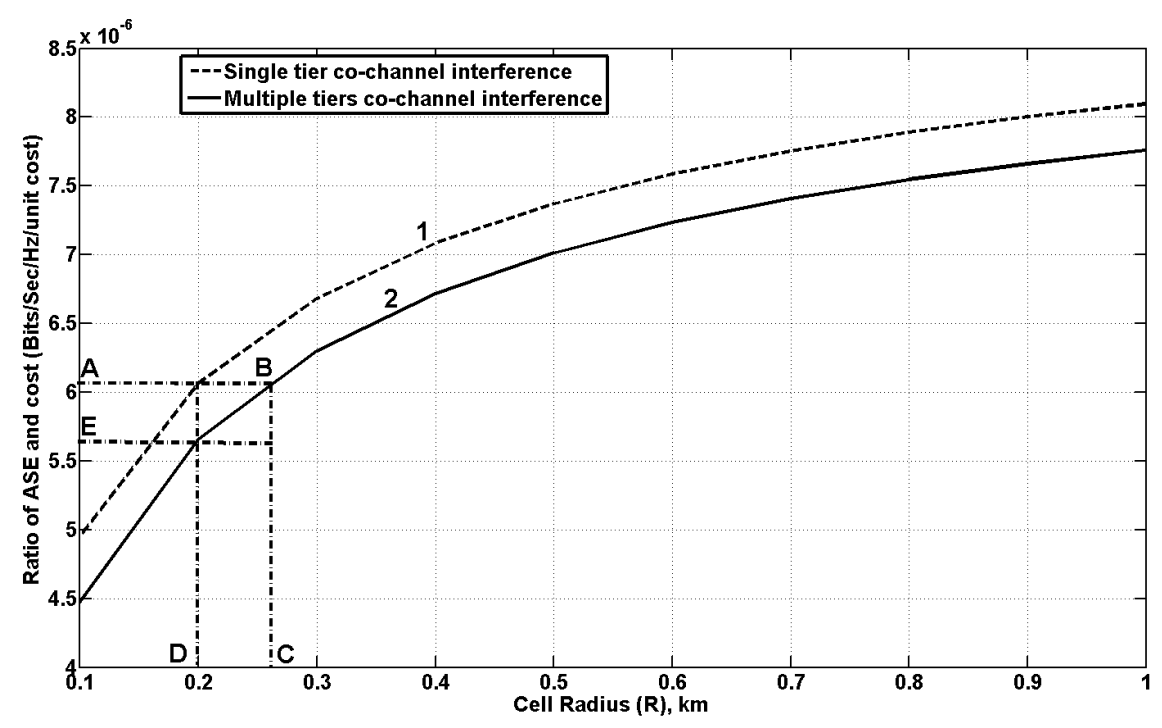

Figure 6.5: Cost comparison between the average uplink area spectral efficiency $A S E$ for single and multiple tier co-channel interference model for different cell radius $R$. Carrier frequency $f_{c}=900 \mathrm{MHz}$; service area $A_{\text {serve }}=1 \mathrm{~km}^{2}$; capital cost; CAPEX $=150000$ per unit cost $(£, \$$, etc) and operation cost $\mathrm{OPEX}=7500$ per unit cost $(£, \$$, etc $)$.

1. Conventional interferers model, ASE per unit cost for single tier model.

2. Proposed interferers model, $A S E$ per unit cost for multiple tier model. 


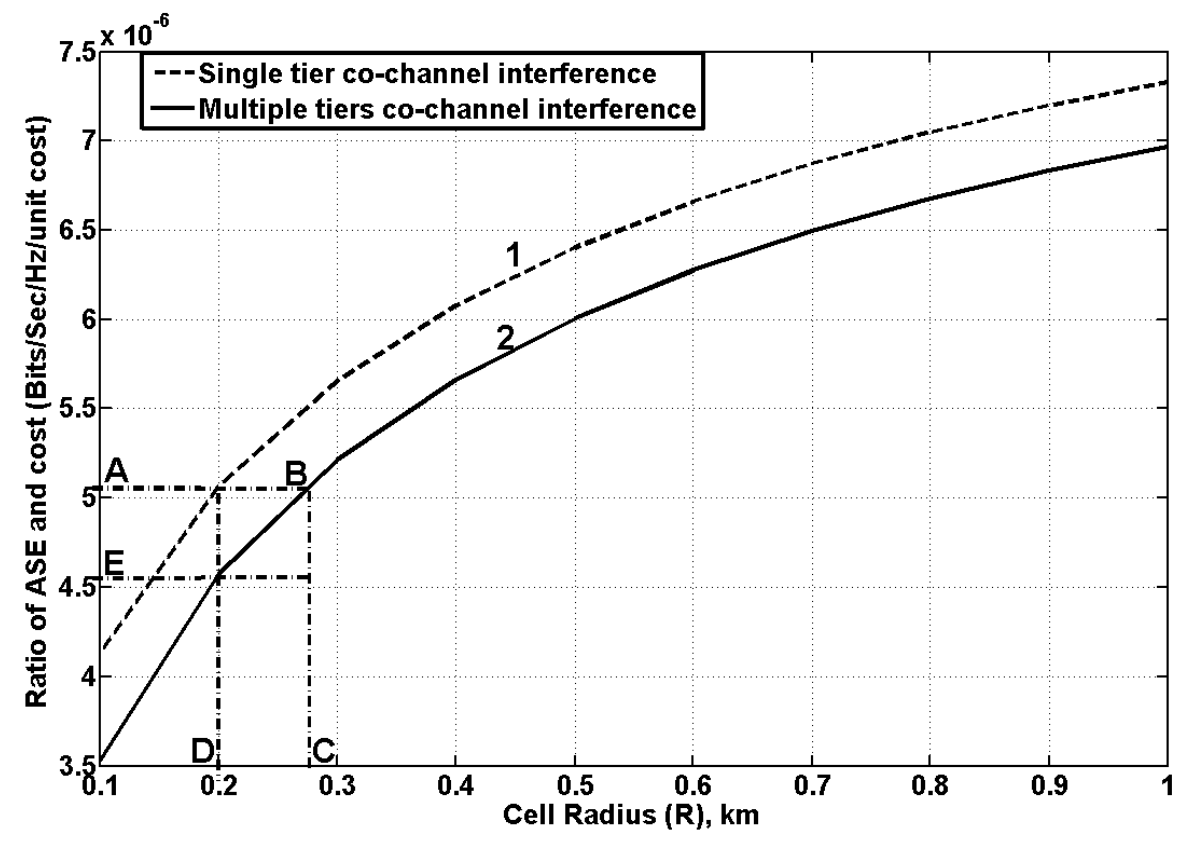

Figure 6.6: Cost comparison between the average uplink area spectral efficiency $A S E$ for single and multiple tier co-channel interference model for different cell radius $R$. Carrier frequency $f_{c}=2 \mathrm{GHz}$; service area $A_{\text {serve }}=1 \mathrm{~km}^{2}$; capital cost; CAPEX $=150000$ per unit cost $(£, \$$, etc $)$ and operation cost $\mathrm{OPEX}=7500$ per unit cost $(£, \$$, etc $)$.

1. Conventional interferers model, ASE per unit cost for single tier model.

2. Proposed interferers model, ASE per unit cost for multiple tier model. 


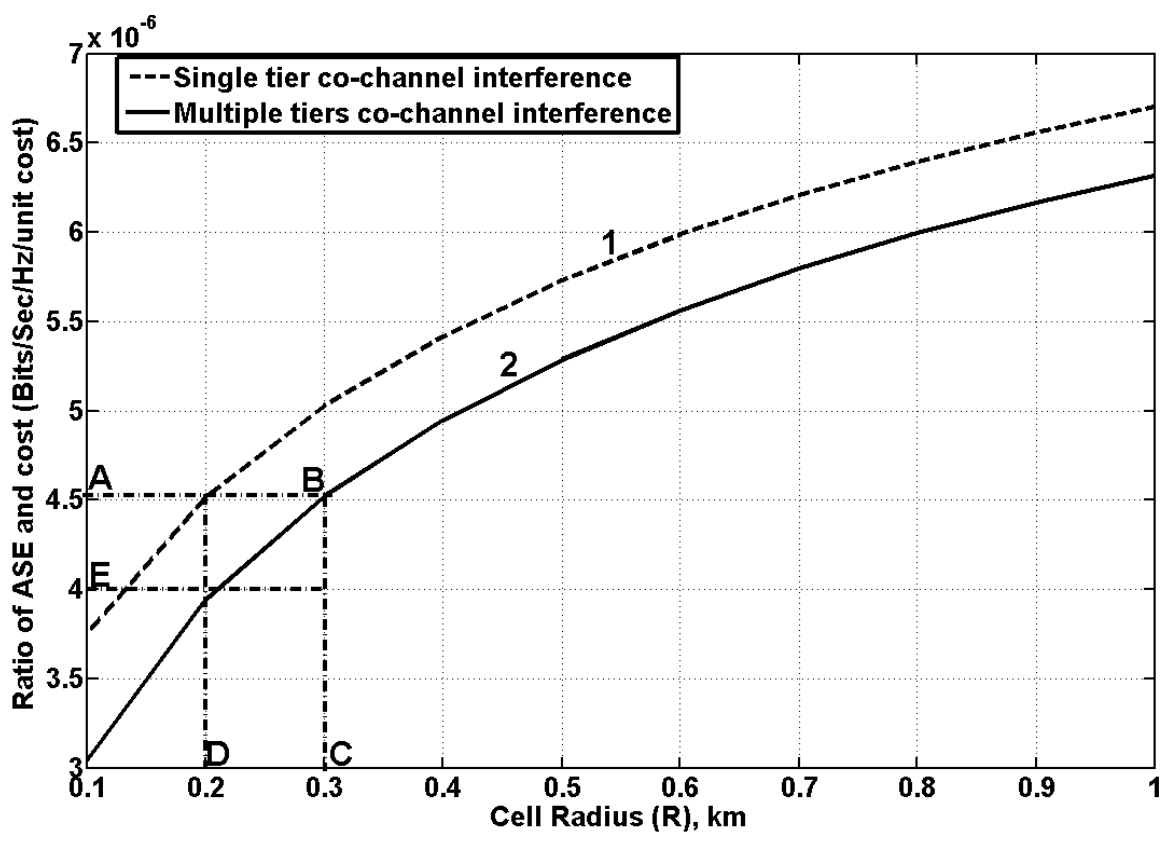

Figure 6.7: Cost comparison between the average uplink area spectral efficiency $A S E$ for single and multiple tier co-channel interference model for different cell radius $R$. Carrier frequency $f_{c}=3.35 \mathrm{GHz}$; service area $A_{\text {serve }}=1 \mathrm{~km}^{2}$; capital cost; CAPEX $=150000$ per unit cost $(£, \$$, etc) and operation cost $\mathrm{OPEX}=7500$ per unit cost $(£, \$$, etc).

1. Conventional interferers model, ASE per unit cost for single tier model.

2. Proposed interferers model, ASE per unit cost for multiple tier model. 


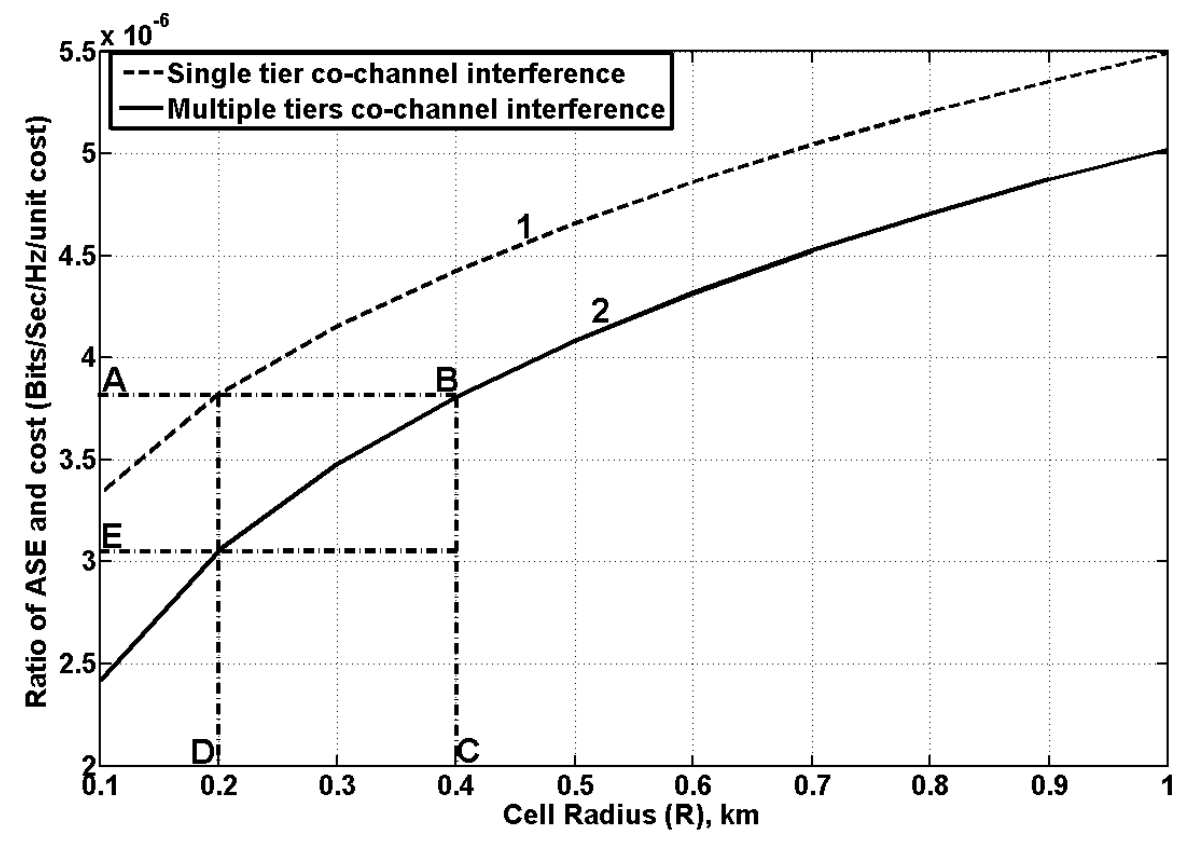

Figure 6.8: Cost comparison between the average uplink area spectral efficiency $A S E$ for single and multiple tier co-channel interference model for different cell radius $R$. Carrier frequency $f_{c}=8.45 \mathrm{GHz}$; service area $A_{\text {serve }}=1 \mathrm{~km}^{2}$; capital cost; CAPEX $=150000$ per unit cost $(£, \$$, etc $)$ and operation cost OPEX $=7500$ per unit cost $(£, \$$, etc).

1. Conventional interferers model, $A S E$ per unit cost for single tier model.

2. Proposed interferers model, ASE per unit cost for multiple tier model. 


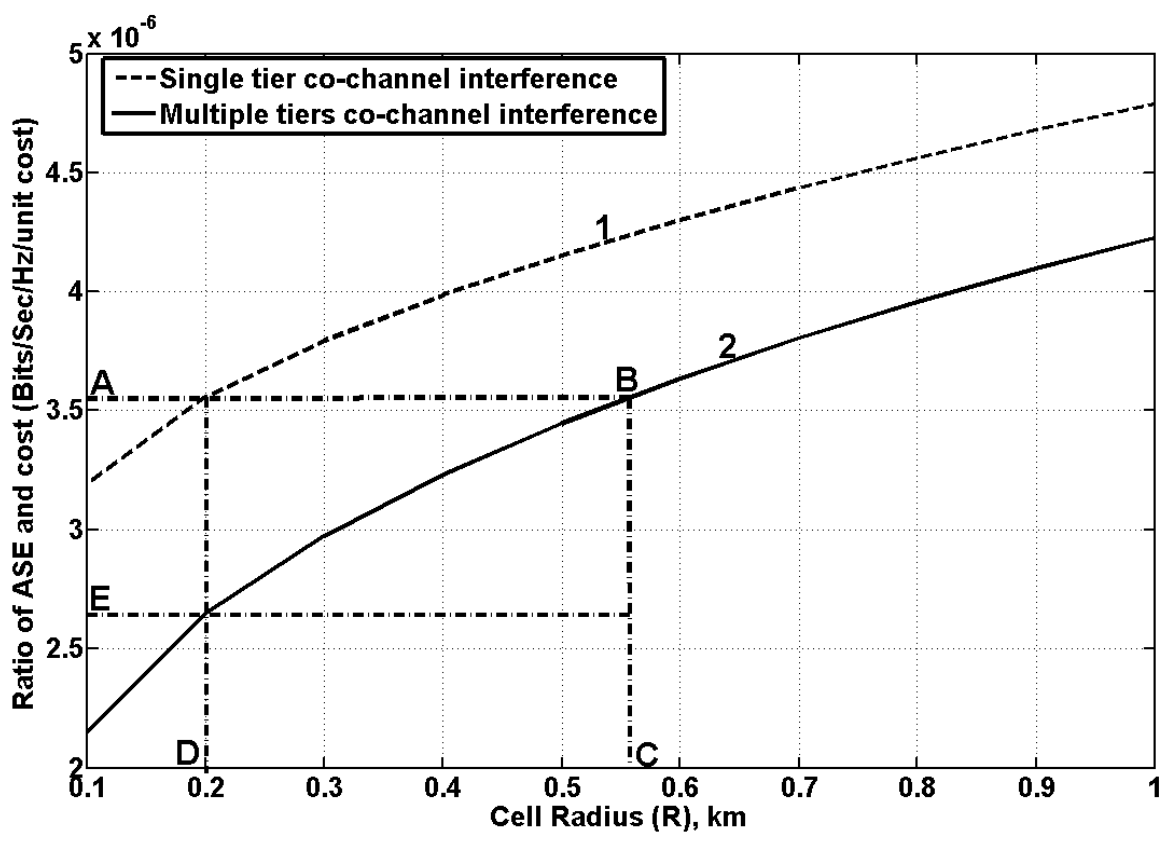

Figure 6.9: Cost comparison between the average uplink area spectral efficiency $A S E$ for single and multiple tier co-channel interference model for different cell radius $R$. Carrier frequency $f_{c}=15.75 \mathrm{GHz}$; service area $A_{\text {serve }}=1 \mathrm{~km}^{2}$; capital cost; CAPEX $=150000$ per unit cost $(£, \$$, etc $)$ and operation cost OPEX $=7500$ per unit cost $(£, \$$, etc).

1. Conventional interferers model, ASE per unit cost for single tier model.

2. Proposed interferers model, $A S E$ per unit cost for multiple tier model. 
$R=200 \mathrm{~m}$, the information capacity per unit cost is $6.06 \times 10^{-6}$, and $5.659 \times 10^{-6}$ [Bits/sec/Hz/unit cost], for the single, and multiple tier interference model. This is represented on Figure 6.5, by the points A, and E. For $f_{c}=3.35 \mathrm{GHz}$, at cell size radius $R=200$ $\mathrm{m}$, the information capacity per unit cost is $4.52 \times 10^{-6}$, and $3.94 \times 10^{-6}[\mathrm{Bits} / \mathrm{sec} / \mathrm{Hz} / \mathrm{unit}$ cost], for the single, and multiple tier interference model. This is also represented on Figure 6.6, by the points A, and E. Finally, when $f_{c}=15.75 \mathrm{GHz}$, at cell size radius $R=200 \mathrm{~m}$, the information capacity per unit cost is $3.56 \times 10^{-6}$, and $2.649 \times 10^{-6}[\mathrm{Bits} / \mathrm{sec} / \mathrm{Hz} / \mathrm{unit}$ cost], for the single, and multiple tier interference model.

The results also show that as cell size radius $R$ reduces, the cell size radius $R$, required to achieve the same information capacity per unit cost, for both interference models, tends to increase for the multiple tier interference model. For example, for $f_{c}=900 \mathrm{MHz}$, the information capacity per unit cost $[\mathrm{Bits} / \mathrm{sec} / \mathrm{Hz} / \mathrm{unit}$ cost $]$ at $R=200 \mathrm{~m}$, for the single tier interference model, equals that at $260 \mathrm{~m}$, for the multiple tier interference model. This is represented in Figure 6.5, by the points C, and D. When carrier frequency $f_{c}=3.35$ $\mathrm{GHz}$, the information capacity per unit cost $[\mathrm{Bits} / \mathrm{sec} / \mathrm{Hz} / \mathrm{unit}$ cost] at cell size radius $R$ $=200 \mathrm{~m}$, for the single tier interference model equals that at cell size radius $R=300 \mathrm{~m}$, for the multiple tier interference model. For carrier frequency $f_{c}=15.75 \mathrm{GHz}$, the information capacity per unit cost [Bits/sec/Hz/unit cost] at cell size radius $R=200 \mathrm{~m}$, for the single tier interference model equals that at cell size radius $R=560 \mathrm{~m}$, for the multiple tier interference model.

These results show that, at higher carrier frequencies, when multiple tier co-channel interference becomes active, there is a limit to cell size radius reduction. There is a limit, because the information capacity gain at a smaller cell size radius, will not be commensurate to the cost invested in obtaining that information capacity gain. The results also indicate that the theoretical limit to cell size radius reduction in cellular systems depends on the carrier frequency.

Figure 6.10 shows a graph of cost per information capacity versus cell size radius $R$, for both interference model at carrier frequency $f_{c}=8.45 \mathrm{GHz}$. The curves indicate that, between 0.4 to $1 \mathrm{~km}$ the cost per information capacity for both interference model remains almost the same. However, for $R \leq 0.3 \mathrm{~km}$ there tend to be an increase in the cost per information capacity for the multiple tier interference model. This implies that 


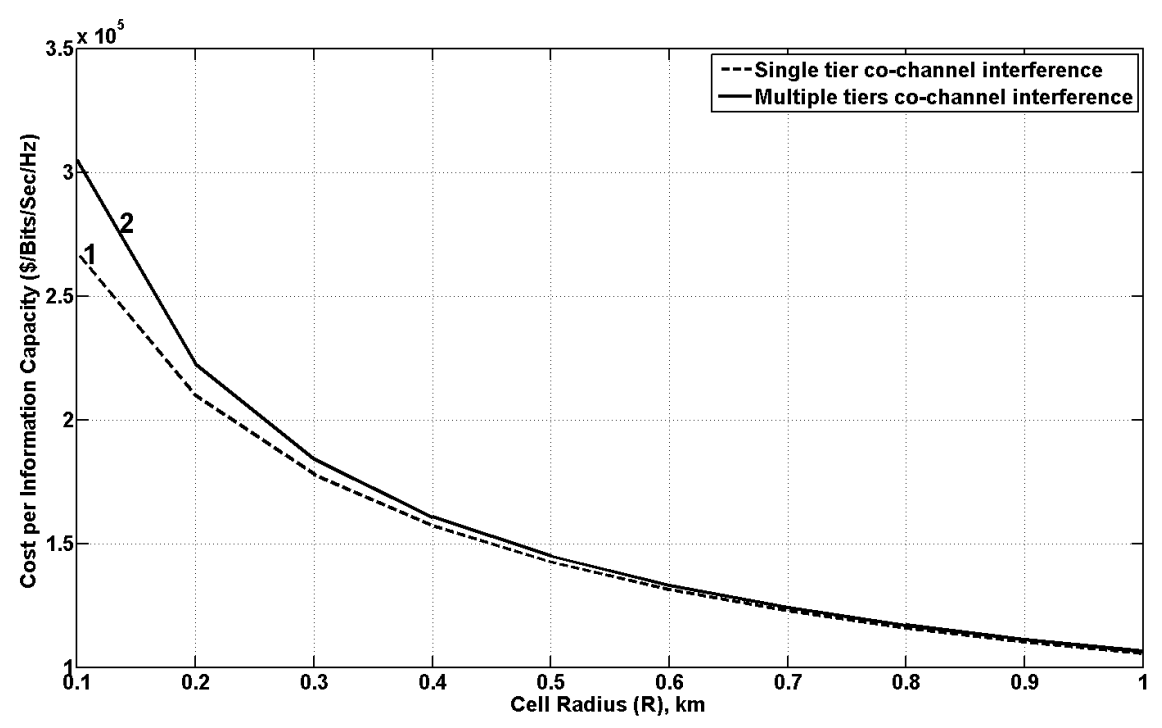

Figure 6.10: Cost per information capacity comparison between single and multiple tier co-channel interference model for different cell radius $R$. Carrier frequency $f_{c}=8.45 \mathrm{GHz}$; service area $A_{\text {serve }}=1 \mathrm{~km}^{2}$; capital cost; CAPEX $=150000$ per unit cost ( $£, \$$, etc) and operation cost OPEX $=7500$ per unit cost $(£, \$$, etc $)$.

1. Conventional interferers model, cost per information capacity for single tier model.

2. Proposed interferers model, cost per information capacity for multiple tier model. 
Wireless Network

the theoretical limit for cell size radius reduction ranges between 0.3 to $0.4 \mathrm{~km}$ for $f_{c}=$ $8.45 \mathrm{GHz}$.

\subsection{Wireless Network}

The wireless possible future development direction is to provide a broader range of wireless product, higher data rates and quality. The previous chapters show that for cellular wireless systems, as carrier frequencies increases and cell size radius decreases other tier co-channel interference becomes active. This leads to a decrease in the information capacity performance of the wireless system. We now consider current development in cellular mobile communication technologies and relate it to our work. We begin this section by outlining some of the current developed cellular mobile communication technologies. We then examine the frequency reuse strategy be employed by this current networks.

We conclude this section with a brief elaboration and comment on how our research work can be applied to this current developed cellular mobile communication technologies.

\subsubsection{Current Cellular Mobile Communication Technologies}

In this section the current developed cellular technologies are described. More details on these developed technologies can be found in [123-125], and the reference therein.

\section{Long Term Evolution (LTE)}

The long term evolution (LTE) as defined by the 3rd Generation Partnership Project (3GPP), is a highly flexible radio interface; which uses Orthogonal Frequency Division Multiplexing (OFDM) wireless broadband technology. LTE offers (i) high spectral efficiency (ii) low latency and (iii) high peak data rates. LTE leverages the economies of scale of $3 \mathrm{G}$, as well as the ecosystem of infrastructure to provide the highest information capacity performance in a cost effective manner. LTE supports more advanced multi - antenna schemes. LTE also supports both Frequency Division Duplex (FDD) and Time Division Duplex (TDD). The information capacity performance targets for both LTE downlink and uplink 
Wireless Network

have been tabulated in [125].

\section{Long Term Evolution - Advanced (LTE - Advanced)}

Comparing the system performance of $3 \mathrm{G}$ and its evolution to LTE, LTE does not offer anything unique to improve spectral efficiency that is the $\mathrm{bps} / \mathrm{Hz}$ [126]. In order to enhance the overall system performance of the network, LTE - Advanced, (that is LTE Release 10) proposed the use of advanced technologies [124]. In other words, LTE - Advanced needs to provide a uniform user experience to users anywhere inside a cell by changing the topology of the conventional networks. This is achieve by shrinking cells size radius, by deployment of a diverse set of base station such as Macrocell, Microcell, Femtocell, Picocell and Relays. This key aspect of the LTE Advanced deployment strategy is termed heterogeneous networks. The research question in this thesis has been - at higher carrier frequencies and smaller cell size radius does other tier co-channel interference become active? In the proceeding sections, an elaboration on the relevancy of the research question to current/latest development in cellular mobile communications are presented. We also give a brief analysis and description on how our research work could be applied to this current/latest development in cellular mobile communications.

\subsubsection{Heterogeneous Networks}

In this section, we give a brief description of (i) conventional cellular wireless networks site layout deployment, (homogeneous networks) and (ii) the alternative and current cellular wireless networks site layout approach, heterogeneous networks.

\section{Homogeneous Networks Deployment}

In the previous chapters it has been reported, that conventional cellular wireless networks are deployed as homogeneous networks by using micro-centric planned process. As described earlier in the thesis homogeneous cellular system is a network of base stations in a planned layout and collection of user terminal, in which all the base stations have transmit power levels, and receiver noise floors. For the conventional cellular wireless networks,

all base stations offer unrestricted asses to user terminals in the network, and serve ap- 
Wireless Network

proximately the same number of user terminals, all of which carries similar data flow with similar QoS requirements.

In homogeneous cellular system, the location of base stations are carefully chosen by network planning, and the settings of the base stations are properly configured to ensure maximization of coverage and the control of interference between base stations. For the conventional cellular networks as traffic demand increases and the RF environments changes, as stated earlier in previous chapters, cell splitting or additional carriers are employed to overcome capacity and link budget limitations to maintain uniform user experience. Currently with the dense population in urban areas, site acquisition for conventional base station with big towers are becoming more difficult. Therefore, there was a need for new site layout deployment model, which led to deployment of heterogeneous networks

\section{Heterogeneous Networks Deployment}

Heterogeneous network is a network consisting of infrastructure points with various wireless access technologies, each of them having different capabilities, constraints, and operating functionalities [127]. Basically in heterogeneous networks low-power base stations are distributed throughout macrocell networks. These low-power base stations can be micro, pico, home (femtocells), relays, and distributed antenna systems [124]. The low power base stations are deployed to get rid of coverage holes in the macrocell-only system and improve capacity in hot-spots [126]. While the placement of macrocell base stations are based on careful planning, the placement of low power base stations may be ad hoc, based only on a rough knowledge of coverage issues and traffic density in the network. Figure 6.11 depicts a heterogeneous cellular wireless network.

Unlike the conventional cellular network, where each mobile terminal is served by the base station with the strongest signal strength, while the unwanted signals received from other base stations are taken care of as interference. In heterogeneous network, the inter tier and intra tier interference problem becomes challenging, which can lead to suboptimal performance if more advanced efficient techniques is not employed for interference management [126]. 


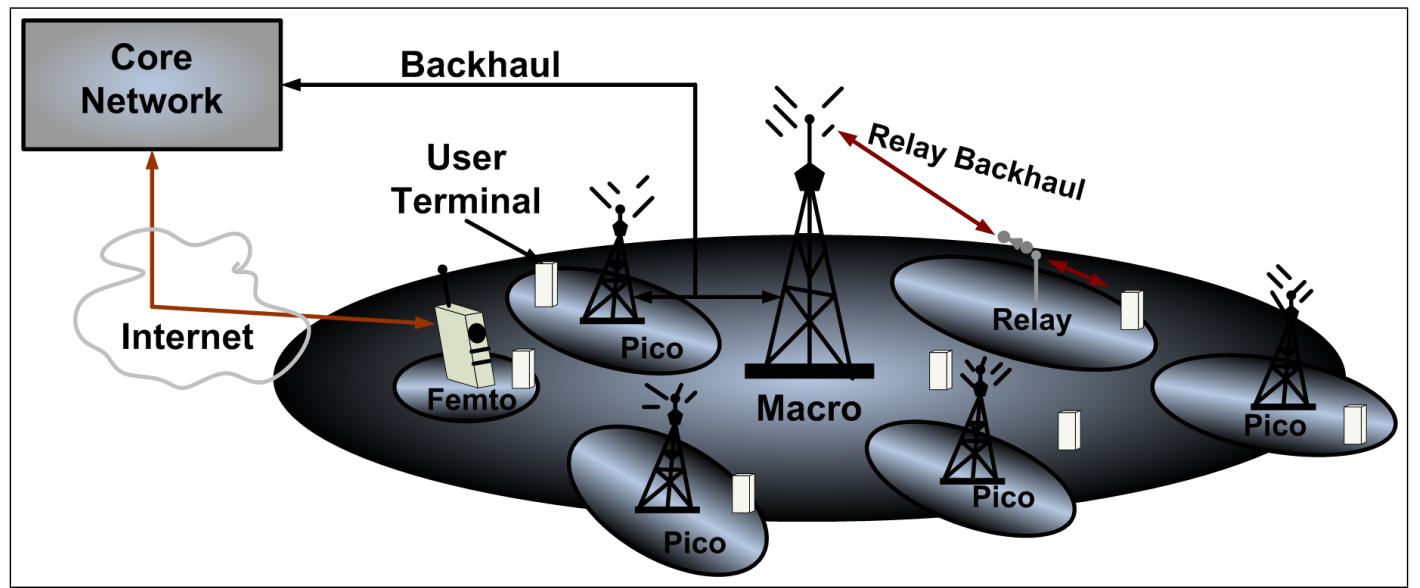

Figure 6.11: Heterogeneous Network, showing a mixture of macro, pico, femto and relay base station [126].

In the following section, we look at some of the interference management techniques employed in heterogeneous networks and relate it to the research question of this thesis.

\subsection{Fractional Frequency Reuse}

In the previous chapters of the thesis, frequency reuse in conventional cellular wireless system was described as the use of the same carrier frequencies $f_{c}$ to cover different geographical areas. The different geographical areas are separated from each other by sufficient distances so that co-channel interference will be reduced.

Unlike conventional cellular networks, in heterogeneous networks, the inter-cell interference are challenging, because heterogeneous networks are unplanned and consist of a mixture of different cell types [128]. To manage the interference in heterogeneous networks, Fractional Frequency Reuse, $(F F R)$ technique has been proposed for OFDMA based cellular wireless networks [129]. Fractional frequency reuse partitions a cell into two areas: central and edge area [130]. In the central areas, frequency can be reused in every cell, whilst in the edge areas, frequency cannot be used in adjacent cells [131]. Basically for fractional frequency reuse, a reuse factor 1 is use at the cell centres where interference is low, and a reuse factor of $1 / 3$ at the cell edge. It have been shown that FFR can greatly improve the spectrum efficiency for a OFDMA based cellular system [132]. 


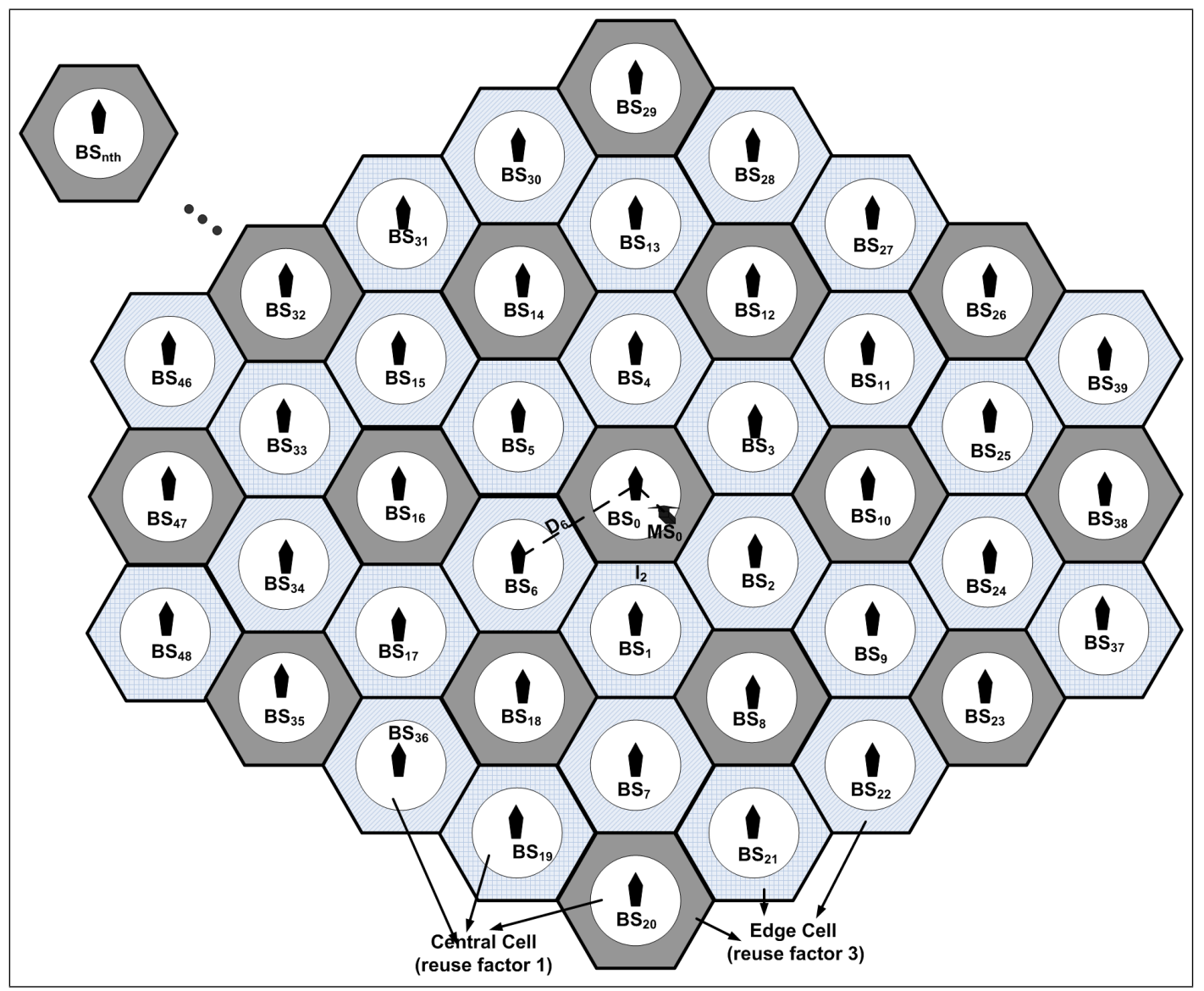

Figure 6.12: A cellular system site layout with fractional frequency reuse [131]. 
A current developed cellular mobile communication system site layout using a reuse factor of $1 / 3$ at edge cells, and a reuse factor of 1 at centre cells is illustrated in Figure 6.12, for the fractional frequency reuse scheme.

LTE, although offers information capacity improvement over $3 \mathrm{G}$ networks, it will still be insufficient to address future expected capacity demands [126]. Therefore acquiring more spectrums and using wider bandwidth would help operates provided more information capacity [133]. The use of FFR in current developed cellular networks leads to natural tradeoffs between improvement in rate and coverage for cell edge users and sum network throughput and spectral efficiency [130]. For operators to address the future expected capacity demands, LTE cellular network may have to be operated at higher carrier frequencies, $f_{c}$, which make our research questions relevant to the latest development in cellular mobile communications. In the next section we will discuss the possible impact of fractional frequency reuse on the minimum cell size radius for information capacity increase in cellular wireless networks.

\subsection{Possible Impact of Fractional Frequency Reuse on the Minimum Cell Size Radius}

Based on the earlier analysis, we study the possible impact of fractional frequency reuse on the information capacity performance in the context of current developed cellular system operating at higher carrier frequencies. Although there are two FFR modes: Strict FFR and Soft Frequency Reuse (SFR) [130], this study focuses on the Strict FFR.

Considering Figure 6.11, the frequency reuse factor in the central area is one, whereas the frequency reuse factor in the edge area is $1 / 3$. The figure shows that the same frequency can be reused among the areas with the same pattern of shadow. For a reference user, $\mathrm{MS}_{0}$ in the central area, $\mathrm{BS}_{0}$, all $\mathrm{MSs}$ outside $\mathrm{BS}_{0}$ may cause co-channel interference through uplink transmission to the reference user. Now comparing the geometrical pattern in Figure 3.7 to Figure 6.12, it can be seen that the proposed formula for calculating the number co-channel interfering cells in a cellular site layout still holds. However the frequency reuse distances will be different as the reuse distance depends on 


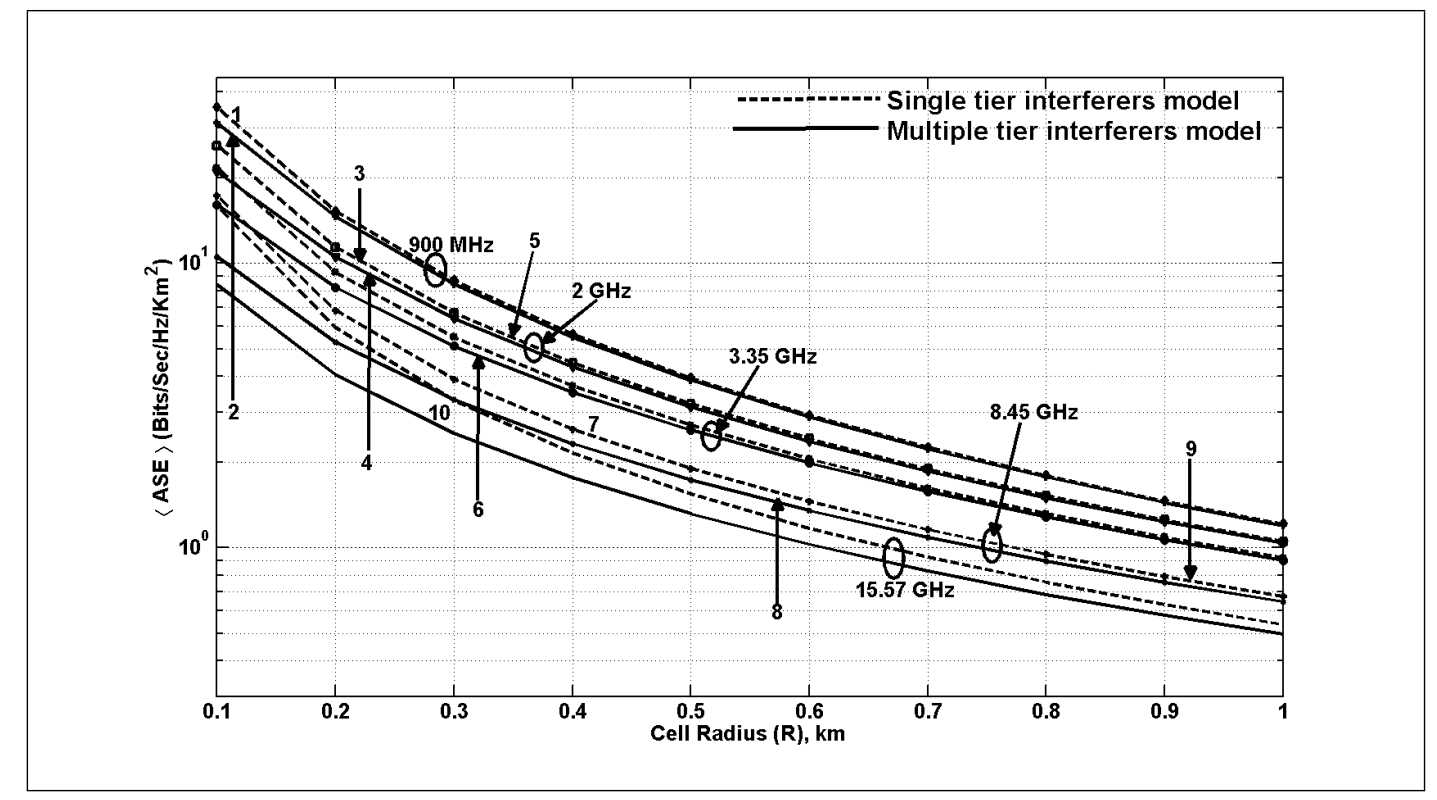

Figure 6.13: Average uplink area spectral efficiency versus cell radius for path loss exponent, $\alpha=2$, and different carrier frequencies $f_{c}$. (Fully-loaded cellular system with co-channel interfering cells in first tier, $N_{F I}=6$, and multiple tier, $N_{N I}=330$; reuse factor $=1 / 3$; extra path loss exponent, $\rho=4$; MS and BS antenna heights : $h_{m}=1.5 \mathrm{~m}$ and $\left.h_{b}=15 \mathrm{~m}\right)$

1 Single tier co-channel interfering model $\left(f_{c}=900 \mathrm{MHz}\right)$ [77]

2 Multiple tier co-channel interfering model $\left(f_{c}=900 \mathrm{MHz}\right)$

3 Single tier co-channel interfering model $\left(f_{c}=2 \mathrm{GHz}\right)[77]$

4 Multiple tier co-channel interfering model $\left(f_{c}=2 \mathrm{GHz}\right)$

5 Single tier co-channel interfering model $\left(f_{c}=3.35 \mathrm{GHz}\right)$ [77]

6 Multiple tier co-channel interfering model $\left(f_{c}=3.35 \mathrm{GHz}\right)$

7 Single tier co-channel interfering model $\left(f_{c}=8.45 \mathrm{GHz}\right)$ [77]

8 Multiple tier co-channel interfering model $\left(f_{c}=8.45 \mathrm{GHz}\right)$

9 Single tier co-channel interfering model $\left(f_{c}=15.75 \mathrm{GHz}\right)[77]$

10 Multiple tier co-channel interfering model $\left(f_{c}=15.75 \mathrm{GHz}\right)$ 


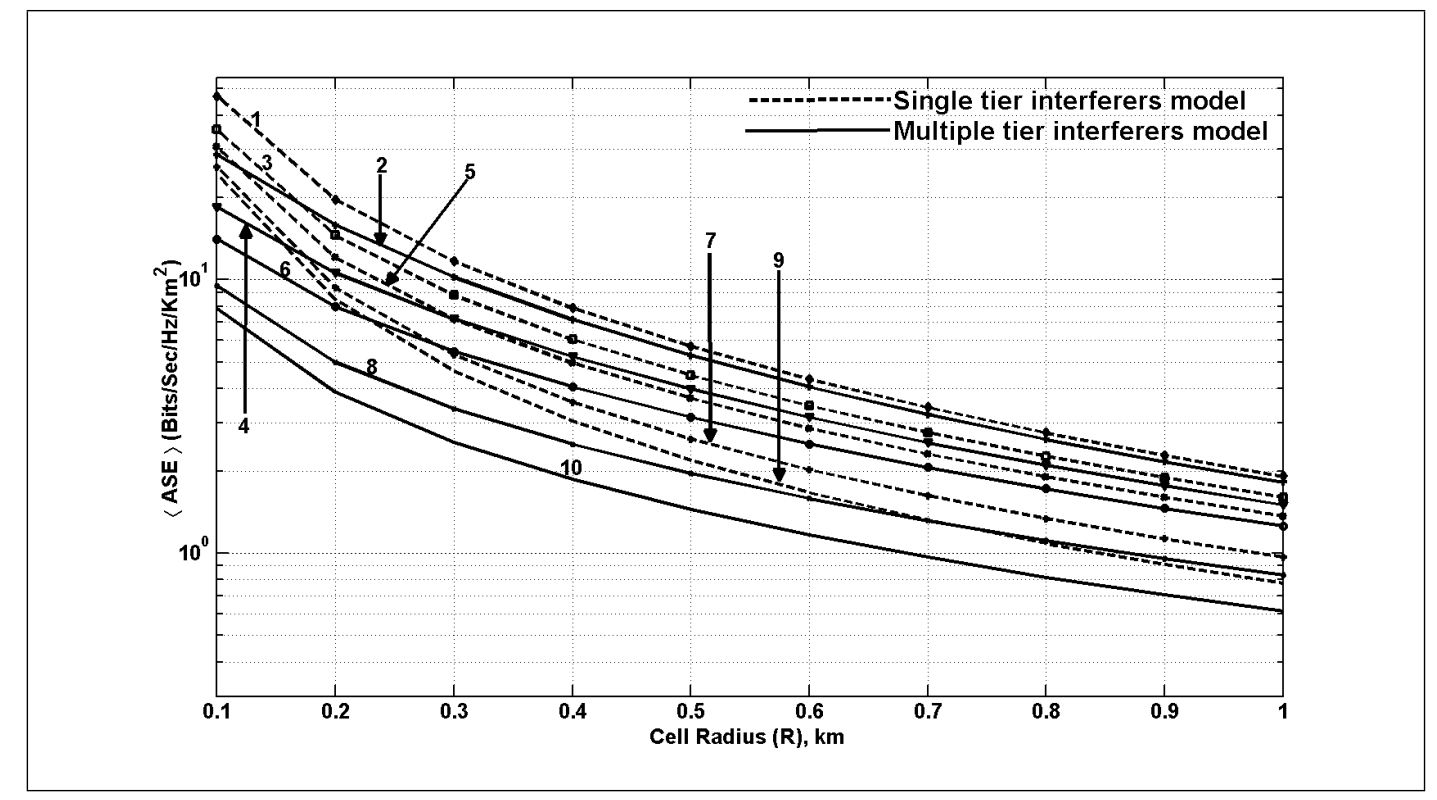

Figure 6.14: Average uplink area spectral efficiency versus cell radius for path loss exponent, $\alpha=2$, and different carrier frequencies $f_{c}$. (Fully-loaded cellular system with co-channel interfering cells in first tier, $N_{F I}=6$, and multiple tier, $N_{N I}=330$; reuse factor $=1$; extra path loss exponent, $\rho=4$; MS and BS antenna heights : $h_{m}=1.5 \mathrm{~m}$ and $h_{b}$ $=15 \mathrm{~m})$

1 Single tier co-channel interfering model $\left(f_{c}=900 \mathrm{MHz}\right)$ [77]

2 Multiple tier co-channel interfering model $\left(f_{c}=900 \mathrm{MHz}\right)$

3 Single tier co-channel interfering model $\left(f_{c}=2 \mathrm{GHz}\right)[77]$

4 Multiple tier co-channel interfering model $\left(f_{c}=2 \mathrm{GHz}\right)$

5 Single tier co-channel interfering model $\left(f_{c}=3.35 \mathrm{GHz}\right)$ [77]

6 Multiple tier co-channel interfering model $\left(f_{c}=3.35 \mathrm{GHz}\right)$

7 Single tier co-channel interfering model $\left(f_{c}=8.45 \mathrm{GHz}\right)$ [77]

8 Multiple tier co-channel interfering model $\left(f_{c}=8.45 \mathrm{GHz}\right)$

9 Single tier co-channel interfering model $\left(f_{c}=15.75 \mathrm{GHz}\right)[77]$

10 Multiple tier co-channel interfering model $\left(f_{c}=15.75 \mathrm{GHz}\right)$ 
the reuse factor.

In the information capacity analysis/simulation a single user was considered. For an OFDMA based system it is assumed that each time/frequency subband is allocated to a single user per cell [134], therefore the analysis/simulations in Chapter 5, can be applied to current developed mobile technologies. To indicate whether our approach can be applied to current developed mobile cellular system a simulation was run following the procedure in Section 5.6, however a reuse factor of one and 1/3 were considered.

Figure 6.13 and 6.14 shows the area spectral efficiency $A S E$ as a function of the cell size radius $R$, for different carrier frequency $f_{c}$ and reuse factor $1 / 3$ and one. Comparing the two figures, Figure 6.14 show an increase in $A S E$ as expected, as the number of available resources depends on the reuse factor [134]. The figures show that as carrier frequencies increases and cell size radius reduces the decrease in information capacity between the two interference model increases. The decrease in information capacity is due to other tier co-channel interference becoming active as the carrier frequency increases and cell size radius reduces. The decrease in information capacity between the two interference model tends to increase for higher reuse factor, because the reuse distance between cochannel interfering cells decreases as the reuse factor becomes higher.

\subsection{Summary}

In this chapter, because the information capacity alone, could not be applied directly to find the minimum cell size radius, for information capacity increase in cellular wireless communication systems. An information capacity/cost approach was used in finding the minimum cell size radius. The information capacity/cost was therefore, defined as the data rate $/ \mathrm{Hz} / \mathrm{km}^{2} /$ unit cost, with the effects of interference included in the data rate calculation. We computed the information capacity/cost ratio for a conventional interference model, and our proposed interference model. We show that at $900 \mathrm{MHz}$, the information capacity/cost for the conventional interference and our proposed interference model were almost the same at cell size radius $R=230 \mathrm{~m}$. However, in the case of $15.75 \mathrm{GHz}$, the information capacity/cost for the conventional model at $R=200 \mathrm{~m}$, equals that of the proposed model at $R=560 \mathrm{~m}$. This result shows that, there is a limit to cell size radius 
reduction, in terms of information capacity gain, and cost, as carrier frequency increases, and as cell size radius decreases.

After outlining some of the current and propose development in cellular mobile communication technologies, we discuss and elaborate on how this research work can be applied to the current and proposed development, and its relevance to the research question in this thesis. However it must be stated that, the discussion does not give a definitive answer to the research question of this thesis, but rather outlines how our approach can be applied to current and proposed cellular mobile communication technologies. 


\section{Chapter 7}

\section{Conclusions and Future Work}




\subsection{Conclusions}

Cellular wireless networks are one of the most useful wireless communication system. The design, and planning of cellular wireless networks has the objective of increasing the information capacity of the systems. Now the most improvement of cellular wireless system information capacity has come from reducing cell size radius, by installing more base stations. However, in the center of Tokyo, when cell size radius was reduced to 300 $\mathrm{m}$ and less there was a problem of co-channel interference. The philosophy of this thesis is therefore to "answer the questions":

- why does co-channel interference, become a problem as cell size radius reduces? and

- is there a theoretical limit to cell size radius reduction for information capacity increase in cellular wireless system in terms of information capacity/cost?

In view of the significance of co-channel interference on the information capacity of cellular wireless communication systems, in Chapter 3, using an inductive approach we have generated a formula for calculating the number of co-channel interfering cells beyond the first tier, for a cellular wireless system site layout. The generated formula shows that the number of co-channel interfering cells in a given tier of a cellular wireless system site layout, regardless of the tessellation, is the product of the number of co-channel interfering cells in the first tier, and the tier number. We proposed a carrier-to-interference model, which includes the first and second tier co-channel interfering cells.

From Chapter 4, we conclude that at carrier frequencies greater than $2 \mathrm{GHz}$ and smaller cell size radius, second tier co-channel interference becomes active. This leads to co-channel interference becoming severe in the cellular network, therefore leading to a decrease in the information capacity of the cellular wireless system. We also concluded that as the basic path loss exponent $\alpha$, decreases the second tier co-channel interference becomes more severe. We found out that at higher frequencies, and smaller cell size radius, when the second tier co-channel interference become active, the information capacity of the cellular wireless system is sensitive to propagation loss and system parameters. 
In Chapter 5, based on the conclusion in Chapter 4, we looked at the case of multiple tier co-channel interfering cells. From Chapter 5, it was found that at higher carrier frequencies, because the breakpoint location is further away from the transmitting source, for smaller cell size radius cellular systems, multiple tiers of co-channel interfering cells are in the free space region. In the free space region, the received signal power decreases in inverse proportion to the second power of distance. However, after the breakpoint, the received signal power decreases in inverse proportion to the fourth power of distance, which renders interference in that region negligible. We conclude that at higher carrier frequencies and smaller cell size radius, multiple tiers of co-channel interfering cells become active. The multiple tier co-channel interfering cells, therefore, cause interference to be more severe, which leads to a decrease in information capacity. The decrease is greater in the case of multiple tiers interference as compared to the first, and second tier co-channel interference cells case. We also found that the information capacity is still sensitive to propagation loss and system parameters.

The main conclusion, to draw from Chapter 6, is that as carrier frequency increases it is not cost effective to reduce the cell size radius below a certain limit. Therefore, for emerging and future cellular wireless communication systems, which will be operating at carrier frequencies greater than $2 \mathrm{GHz}$, it will not be advisable to reduce the cell size radius indefinitely in terms of cost and the information capacity gain of the cellular wireless network. The theoretical limit for the cell size radius reduction depends on the carrier frequency. For example at a carrier frequency of $8.45 \mathrm{GHz}$ the theoretical limit for cell size radius reduction for information capacity increase in terms of cost per information capacity ranges from 0.3 to $0.4 \mathrm{~km}$.

\subsection{Future Work}

While this thesis provides an in depth insight into (i) the problem of characterizing cochannel interference in cellular systems, operating at carrier frequencies greater than 2 $\mathrm{GHz}$, and smaller cell size radius (ii) the information capacity performance of emerging cellular systems, and finally, (iii) finding a minimum cell size for information capacity increase in emerging cellular systems, a variety of fruitful areas remain for future research. 
We emphasize the following major interrelated research directions.

In order to estimate the information capacity of the cellular wireless network, we have presented a simplified propagation model. While it can be argued that the accuracy of the simplified model (proposed in Chapter 3), used for our analysis and simulation may be poor. For future work, we suggest the development of a new model. The new model, should not be significantly more complex than our simplified model, yet should incorporates shadowing effects, small-scale fading, and noise.

It is worth pointing out that the proposed co-channel interference model, for cellular wireless communication networks operating at carrier frequencies greater than $2 \mathrm{GHz}$, may be further improved to be realistic in various ways:

- Information capacity performance analysis for different statistical path loss models scenarios, such as indoor, and outdoor users connecting to an indoor or outdoor base station.

- Information capacity performance analysis for non-uniformly distributed mobile users, using the proposed interference model.

It will also be interesting to characterize the co-channel interference, for the case of downlink, sectorized, and other multiple access scheme, such as code division multiple access (CDMA) cellular wireless communication systems. The developed cost model may be further improved to be realistic in various ways:

- When different BS station types are considered for the infrastructure cost model.

- When net revenue generated per cell per year, and the profit generated per cell per year is also included in the analysis.

It will also be valuable to consider a three dimension interference model, as most cities are now filled with highrise building, therefore cell sites may be separated in three dimensions. Consequently interference in city centres may be in three dimensions. Regarding the minimum cell size radius, it would be interesting if a definite optimal cell size radius that maximizes the information capacity of the cellular wireless communication were obtained. It is important for one to confirm whether the simulation results shown 
Future Work

in this thesis continue to hold for Poisson distributed cells [135], which have been shown recently to be a better model, for interference in cellular wireless communication systems.

Finally this work would have been more valuable if the simulated result were compared with experimentally observed results. Unfortunately it will be very expensive to carry out such an experiment. 
BIBLIOGRAPHY 


\section{Bibliography}

[1] W. C. Jakes, Microwave mobile communications. New York, NY: John Wiley \& Sons, 1994, pp. $100-142$.

[2] J. Takada, J. Fu, H. Zhu, and T. Kobayashi, "Spatio-temporal channel characterization in a suburban non line-of-sight microcellular environment," IEEE J. Select. Areas Commun., vol. 20, no. 3, pp. 532-538, Apr. 2002.

[3] R. Zhang and L. Hanzo, "Wireless cellular networks," Veh. Technolo. Mag., vol. 5, no. 4, pp. $31-39,2010$.

[4] V. H. MacDonald, “The cellular concept,” Bell Syst. Tech. J., vol. 58, no. 1, pp. 15 -41 , Jan. 1979.

[5] P. Harley, "Short distance attenuation measurements at $900 \mathrm{mhz}$ and $1.8 \mathrm{ghz}$ using low antenna heights for microcells," IEEE J. Select. Areas Commun., vol. 7, no. 1, pp. 5 - 11, Jan. 1989.

[6] Y. Liang, A. Goldsmith, G. Foschini, R. Valenzuela, and D. Chizhik, "Evolution of base station in cellular networks: denser deployment versus coordination," in Proc. IEEE International Conference on Communications (ICC'2008), Beijing, China, May 2008, pp. $4128-4132$.

[7] S. Zhou, M. Zhao, X. Xu, J. Wang, and Y. Yao, "Distributed wireless communication system: A new architecture for future public wireless access," IEEE Commun. Mag., vol. 41, pp. $108-113,2003$.

[8] A. J. Schwab and P. Fischer, "Maxwell, hertz, and german radio-wave history," Proc. IEEE, vol. 86, no. 7, pp. 1312 - 1318, Jul. 1998. 
[9] F. Gardiol and Y. Fournier, "Marconi in switzerland true story or fairy tale," in Proc. History of Telecommunications (HISTELCON'2008'), Paris, France, Sept. 2008, pp. $12-19$.

[10] G. R. M. Garrat, The Early History of Radio: from Faraday to Marconi. London, UK: Institution of Engineering and Technology, 1994.

[11] G. C. Corazza, "Marconi’s history,” Proc. IEEE, vol. 86, no. 7, pp. 1307 - 1311, Jul. 1998.

[12] G. B. Giannakis, "Signal processing advances in wireless and mobile communications," IEEE Signal Processing Mag., vol. 17, no. 3, pp. 14 - 15, May 2000.

[13] G. B. Giannakis, P. Stoica, and Y. Hua, Signal Processing Advances in Wireless and Mobile Communications, Volume I: Trends in Channel Estimation and Equalization. Upper Saddle, NJ: Prentice Hall PTR, 2000.

[14] J. Mikkonen, C. Corrado, C. Evci, and M. Progler, "Emerging wireless broadband networks," IEEE Commun. Mag., vol. 36, no. 2, pp. 112 - 117, Feb. 1998.

[15] Y. Oda, K. Tsunekawa, and M. Hata, "Advanced los path-loss model in microcellular mobile communications," IEEE Trans. Veh. Technol., vol. 49, no. 6, pp. 2121 -2125 , Nov. 2000.

[16] T. K. Sarkar, Z. Ji, K. Kim, A. Medouri, and M. Salazar-Palma, "A survey of various propagation models for mobile communication," IEEE Trans. Antennas Propagat., vol. 45, pp. 51 - 74, Jun. 2003.

[17] B. G. Evans and K. Baughan, "Visions of 4g," IET Electronics \& Communication Engineering Journal, vol. 12, no. 6, pp. 293 - 303, Dec. 2000.

[18] M. Dinis and J. Fernandes, "Provision of sufficient transmission capacity for broadband mobile multimedia: a step toward 4g," IEEE Commun. Mag., vol. 39, no. 8, pp. 46 - 54, Aug. 2001.

[19] G. L. Stüber, Principles of mobile communication., 2nd ed. Norwell, MA: Kluwer Academic, 2001, pp. 1 - 3. 
[20] A. Goldsmith, Wireless communication. New York, NY: Cambridge University, 2005 , pp. $27-40$.

[21] A. F. Molisch, Wireless communication. New York, NY: John Wiley \& Sons, 2005 , pp. $1-31$.

[22] A. J. Goldsmith, "Design and performance of high-speed communication systems over time-varying radio channels," $\mathrm{PhD}$ dissertation, University of California at Berkeley, 1994.

[23] J. G. Andrews, "Interference cancellation for cellular systems: A contemporary overview," IEEE Trans. Wireless Commun., vol. 12, no. 2, pp. 19 - 29, Apr. 2005.

[24] Z. Krusevac, "Model-based approach to reliable information transfer over timevarying communication channels," PhD dissertation, Australian National University, 2007.

[25] C. E. Shannon, "A mathematical theory of communication, part i," Bell Syst. Tech. J., vol. 27, pp. $379-423,1948$.

[26] W. C. Y. Lee, "Spectrum efficiency in cellular," IEEE Trans. Veh. Technol., vol. 38, pp. 69 - 75, May 1989.

[27] K. Pahlavan and A. H. Levesque, "Wireless data communication," Proc. IEEE, vol. 82, pp. 1398 - 1430, Sept. 1994.

[28] G. Hernández-Valdez, F. A. Cruz-Pérez, and D. Lara-Rodríguez, "Sensitivity of the system performance to the propagation parameters in los microcellular environments," IEEE Trans. Veh. Technol., vol. 57, no. 6, pp. 3488 - 3508, Nov. 2008.

[29] D. N. Hatfield, "Measures of spectral efficiency in land mobile radio," IEEE Trans. Electromagn. Compat., vol. 19, no. 3, pp. 266 - 268, Aug. 1977.

[30] C. F. Ball and H. K. Ivanov, "Spectrum efficiency evaluation for different wireless technologies based on traffic modelling," in Proc. IEEE 16th IEEE International Symposium on Personal Indoor, and Mobile Radio Communications Workshops, (PIRMC'2005), vol. 3, Sept. 2005, pp. 2055 - 2061. 
[31] G. L. Stüber, Principles of mobile communication, 2nd ed. Norwell, MA: Kluwer Academic, 2001, pp. 28 - 34.

[32] I. H. Cavdar and O. Akcay, "The optimization of cell sizes and base stations power level in cell planning," in Proc. 53rd IEEE Vehicular Technology Conference (VTC'2011), vol. 4, Rhodes, Greece, May 2001, pp. 2344 - 2348.

[33] P. Cardieri and T. S. Rappaport, "Statistical analysis of co-channel interference in wireless communications systems," Wirel. Commun. Mob. Comput., vol. 1, no. 1, pp. 112 - 121, Jan./Mar. 2001.

[34] R. French, "The effect of fading and shadowing on channel reuse in mobile radio," IEEE Trans. Veh. Technol., vol. 28, no. 3, pp. 177 - 181, Aug. 1979.

[35] D. Cox, "Cochannel interference considerations in frequency reuse small-coveragearea radio systems," IEEE Trans. Commun., vol. 30, no. 1, pp. 135 - 142, Jan. 1982.

[36] R. Muammar and S. Gupta, "Co-channel interference in high-capacity mobile radio systems," IEEE Trans. Commun., vol. 30, no. 8, pp. 1973 - 1978, Aug. 1982.

[37] W. C. Lee, "Smaller cells for greater performance," IEEE Commun. Mag., vol. 29, no. 11 , pp. $19-23$, Nov. 1991.

[38] Y. Yao and A. U. H. Sheikh, "Investigations into co-channel interference in microcellular mobile radio systems," IEEE Trans. Veh. Technol., vol. 41, no. 2, pp. 114 - 121, May 1992.

[39] V. Chandrasekhar, J. Andrews, and A. Gatherer, "Femtocell networks: a survey," IEEE Commun. Mag., vol. 46, no. 9, pp. 59 - 67, Sept 2008.

[40] C. J. Ho, J. A. Copeland, C. T. Lea, and G. L. Stuber, "On call admission control in ds/cdma cellular networks," IEEE Trans. Veh. Technol., vol. 50, no. 6, pp. 1328 - 1343, Nov. 2001.

[41] W. C. Y. Lee, "Overview of cellular cdma," IEEE Trans. Veh. Technol., vol. 40, pp. 291 - 302, May 1991. 
[42] L. Závodný and S. Hanus, "Cellular radio network optimization based on the $\mathrm{c} / \mathrm{i}$ ratio and transmission capacity," in Proc. IEEE 2004 High Frequency Postgraduate Student Colloquium, vol. 3, Sept. 2004, pp. 67 - 72.

[43] Z. Kostić, I. Marić, and X. Wang, "Fundamentals of dynamic frequency hopping in cellular systems," IEEE J. Select. Areas Commun., vol. 19, no. 11, pp. 2254 2266, Nov. 2001.

[44] J. Wu, N. Mehta, and J. Zhang, "Spectral efficiency analysis of cellular systems with channel-aware schedulers," in conf. Rec. 2005 IEEE Global Telecom. Conf., vol. 3, p. 5, Nov. 2005.

[45] K. A. Anang, P. B. Rpajic, T. I. Eneh, and Y. Nijsure, "Minimum cell size for information capacity increase in cellular wireless network," in Proc. 73rd IEEE Vehicular Technology Conference (VTC'2011), Budapest, Hungary, May 2011, pp. $305-311$.

[46] K. A. Anang, P. B. Rapajic, T. I. Eneh, and G. Oletu, "Sensitivity of information capacity of land mobile cellular system to the base station antenna height at higher microwave frequencies," in Proc. 3rd International Conference on Computer Research and Development (IEEE- ICCRD 2011), Shanghai, China, Mar. 2011, pp. $167-172$.

[47] K. A. Anang, P. B. Rapajic, T. I. Eneh, and B. Lawal, "Sensitivity of information capacity of land mobile cellular system to propagation loss parameters at higher microwave frequencies," in Proc. 7th IEEE International Wireless Communications and Mobile Computing Conference (IEEE- IWCMC 2011), Istanbul, Turkey, Jul. 2011, pp. $630-635$.

[48] K. A. Anang, P. B. Rapajic, T. I. Eneh, L. Bello, and G. Oletu, "Impact of vehicular traffic on information capacity of cellular wireless network at carrier frequencies greater than $3 \mathrm{ghz}$," in Proc. 5th IEEE European Modelling Symposium on Mathematical modelling and Computer Simulation (EMS' 2011), Madrid, Spain, Nov. 2011, pp. 430 - 434 . 
[49] K. A. Anang, P. B. Rapajic, R. Wu, L. Bello, and T. I. Eneh, "Cellular system information capacity change at higher frequencies due to propagation loss and system parameters," Progress in Electromagnetics Research B, vol. 44, pp. 191 - 221, Sept 2012.

[50] K. A. Anang, P. B. Rapajic, L. Bello, and R. Wu, "Sensitivity of cellular wireless network performance to system \& propagation parameters at carrier frequencies greater than 2 ghz," Progress in Electromagnetics Research B, vol. 40, pp. 31 - 54, Apr. 2012.

[51] K. A. Anang, P. B. Rapajic, T. I. Eneh, and R. Wu, "Minimum cell size for information capacity increase in cellular wireless network," Wireless Communications and Mobile Computing Journal, vol. 44, 2012, accepted.

[52] A. Nešković, N. Nešković, and D. Paunocć, "Macrocell electric field strength prediciton model based upon artificial neural networks," IEEE J. Select. Areas Commun., vol. 20, no. 6, pp. 1170 - 1176, Aug. 2002.

[53] R. Steele and L. Hanzo, Mobile radio communications, 2nd ed. London, UK: Pentech Press, 1992, vol. 3.

[54] N. Blaunstein, Radio propagation in cellular networks, 1st ed. Norwood, MA, USA: Artech House, Inc., 1999.

[55] C. E. Shannon, "A mathematical theory of communication, part ii," Bell Syst. Tech. $J .$, vol. 27, pp. $623-656,1948$.

[56] R. Gallager, Reliable communication. John Wiley \& Sons, 1968.

[57] T. M. Cover and J. A. Thomas, Elements of Information Theory. New York, NY: Wiley, 1991.

[58] G. J. Foschini, "Layered space-time architecture for wireless communication in a fading environment when using multi-element antennas," Bell labs tech. $j$, vol. 1, no. 2 , pp. $41-59,1996$. 
[59] V. Tarokh, N. Seshadri, and A. R. Calderbank, "Space-time codes for high data rate wireless communication: Performance criterion and code construction," IEEE Trans. Inform. Theory, vol. 44, no. 2, pp. 744 - 765, Mar. 1998.

[60] W. C. Y. Lee, Mobile communications Engineering, 2nd ed. New York, NY: McGraw-Hill, 1997, pp. 559 - 570.

[61] — Mobile communication design fundamentals, 2nd ed. New York, NY, USA: John Wiley \& Sons, 1993, p. 142.

[62] M. F. Iskander and Z. Yun, "Propagation prediction models for wireless communication systems," IEEE Trans. Microwave Theory Tech., vol. 50, no. 3, pp. $662-$ 673, Mar. 2002.

[63] M. Hata, "Empirical formula for propagation loss in land mobile radio services," IEEE Trans. Veh. Technol., vol. 29, no. 3, pp. 317 -325, Aug. 1980.

[64] L. Piazzi and H. L. Bertoni, "Achievable accuracy of site-specific path-loss predictions in residential environments," IEEE Trans. Veh. Technol., vol. 48, no. 3, pp. 922 - 930, May 1999.

[65] S. C. Kim, B. J. Guarino, T. M. Willis, V. Erceg, S. J. Fortune, R. A. Valenzuela, L. W. Thomas, J. Ling, and J. D. Moore, "Radio propagation measurements and prediction using three-dimensional ray tracing in urban environments at $908 \mathrm{mhz}$ and 1.9 ghz," IEEE Trans. Veh. Technol., vol. 48, no. 3, pp. 931 - 946, May 1999.

[66] Y. L. C. D. Jong and M. Herben, "Prediction of local mean power using 2-d raytracing-based propagation models," IEEE Trans. Veh. Technol., vol. 50, no. 1, pp. 325 - 331, Jan 2001.

[67] R. P. Torres, S. Loredo, L. Valle, and M. Domingo, "An accurate and efficient method based on ray-tracing for the prediction of local flat-fading statistics in picocell radio channels," IEEE J. Select. Areas Commun., vol. 19, no. 2, pp. 170 - 178, Feb. 2001. 
[68] H. H. Xia, H. L. Bertoni, L. R. Maciel, A. Lindsay-Stewart, and R. Rowe, "Radio propagation characteristics for line-of-sight microcellular and personal communications," IEEE Trans. Antennas Propagat., vol. 41, no. 10, pp. 1439 - 1447, Oct. 1993.

[69] Y. Oda, K. Tsuunekawa, and M. Hata, “Advanced los path-loss model in microcellular mobile communications," IEEE Trans. Veh. Technol., vol. 49, no. 6, pp. 2121 -2125 , Nov. 2000.

[70] W. C. Jakes, Microwave mobile communications. New York, NY, USA: Wiley \& Sons, 1974.

[71] N. Amitay, "Modelling and computer simulation of wave propagation in lineal lineof-sight microcells," IEEE Trans. Veh. Technol., vol. 41, no. 4, pp. 337 -342, Nov. 1992.

[72] A. J. Rustako, N. Amitay, G. J. Owens, and R. S. Roman, "Radio propagation at microwave frequencies for line-of-sight microcellular mobile and personal communications," Vehicular Technology, IEEE Transactions on, vol. 40, no. 1, pp. 203 210, Feb. 1991.

[73] T. K. Sarkar, Z. Ji, K. Kim, A. Medouri, and M. Salazar-Palma, "A survey of various propagation models for mobile communication," IEEE Trans. Antennas Propagat., vol. 45, no. 3, pp. 51 - 82, Jun. 2003.

[74] R. Coombs and R. Steele, "Introducing microcells into macrocellular networks: A case study," IEEE Trans. Commun., vol. 47, no. 4, pp. 568 - 576, Apr. 1999.

[75] S. O. Rice, "Mathematical analysis of random noise,"” Bell Syst. Tech. J., vol. 23, no. 7 , pp. $282-333$, Jul. 1944.

[76] F. A. Cruz-Pérez, D. Lara-Rodríguez, and M. Larar, "Full-and half-square cell plans in urban cdma microcellular networks," IEEE Trans. Veh. Technol., vol. 52, no. 3, pp. $502-511$, May 2003. 
[77] M. Alouini and A. J. Goldsmith, "Area spectral efficiency of cellular mobile radio systems," IEEE Trans. Veh. Technol., vol. 48, no. 4, pp. 1047 - 1065, Jul. 1999.

[78] M. Pratesi, F. Santucci, and F. Graziosi, "Generalized moment matching for the linear combination of lognormal rvs: application to outage analysis in wireless systems," IEEE Trans. Wireless Commun., vol. 5, no. 5, pp. 1122 - 1132, May 2006.

[79] X. Ge, K. Huang, C. X. Wang, X. Hong, and X. Yang, "Capacity analysis of a multi-cell multi-antenna cooperative cellular network with co-channel interference," IEEE Trans. Wireless Commun., vol. 10, no. 10, pp. 3298 -3309, Oct. 2011.

[80] M. V. Clark, V. Erceg, and L. J. Greenstein, "Reuse efficiency in urban microcellular networks," IEEE Trans. Veh. Technol., vol. 46, no. 2, pp. 279 - 288, May 1997.

[81] M. G. Sánchez, I. Cuinas, and A. V. Alejos, "Electromagnetic field level temporal variation in urban areas," IET Electronics Letters, vol. 41, no. 5, pp. 233 - 234, Mar 2005.

[82] Y. Liang, Y. Taesang, and A. Goldsmith, "Coverage spectral efficiency of cellular systems with cooperative base stations," in Fortieth Asilomar Conference on Signals, Systems \& Computers, California, USA, Oct. 2006, pp. 349 - 353.

[83] K. Kitao and S. Ichitsubo, "Path loss prediction formula for microcell in $400 \mathrm{mhz}$ to 8 ghz band," Electronics Letters, vol. 40, no. 11, pp. 685 - 687, May 2004.

[84] A. Domazetovic, L. J. Greenstein, N. B. Mandayam, and I. Seskar, "Propagation models for short-range wireless channels with predictable path geometries," IEEE Trans. Commun., vol. 53, no. 7, pp. 1123 - 1126, Jul. 2005.

[85] H. Masui, T. Kobayashi, and M. Akaike, "Microwave path-loss modelling in urban line-of-sight environments," IEEE J. Select. Areas Commun., vol. 20, no. 6, pp. $1151-1155$, Aug. 2002.

[86] D. C. Cox, R. R. Murray, and A. W. Norris, "800-mhz attenuation measured in 
and around suburban houses," AT\&T Bell Laboratories technical journal, vol. 63, no. 6, pp. $921-954$, Nov. 1984.

[87] ITU, "Propagation data and prediciton methods for planning of short-range outdoor radiocommunication systems and radio local area networks in the frequency range 300 mhz to 100 ghz," Recommendation ITU-R P.1411 - 1, iTU Radiocommunication Assembly.

[88] S. Singh, N. B. Mehta, A. F. Molisch, and A. Mukhopadhyay, "Moment-matched lognormal modelling of uplink interference with power control and cell selection," IEEE Trans. Wireless Commun., vol. 9, no. 3, pp. 932 - 938, Mar. 2010.

[89] J. Wang and L. B. Milstein, "Cdma overlay situations for microcellular mobile communications," IEEE Trans. Commun., vol. 43, no. 234, pp. 603 - 614, May 1995.

[90] —_ "Approximate interference of a microcellular spread spectrum system," Electronics Letters, vol. 31, no. 20, pp. 1782 - 1783, Sept. 1995.

[91] J. Arnbak and W. V. Blitterswijk, "Capacity of slotted aloha in rayleigh-fading channels," IEEE J. Select. Areas Commun., vol. 5, no. 2, pp. 261 - 269, Feb. 1987.

[92] R. Prasad and A. Kegel, "Improved assessment of interference limits in cellular radio performance," IEEE Trans. Veh. Technol., vol. 40, no. 2, pp. 412 -419, May 1991.

[93] S. Phaiboon and P. Phokharatkul, "Path loss prediction for low-rise buildings with image classification on 2-d aerial photographs," Progress In Electromagnetics Research, vol. 95, pp. 135 - 152, 2009.

[94] D. C. Cox, R. R. Murray, and A. W. Norris, "Antenna height dependence of 800 mhz attenuation measured in houses," IEEE Trans. Veh. Technol., vol. 34, no. 2 , pp. 108 - 115, May 1985.

[95] E. Green, "Radio link design for microcellular systems," British Telecom technology, vol. 8, no. 1, 1990. 
[96] F. A. Cruz-Perez and D. Lara-Rodriguez, "Impact of the breakpoint distance on the reverse link capacity of a cdma manhattan like microcellular system," in Proc. 5th IEEE International Symposium on Spread Spectrum Techniques and Applications (ISSSTA'1998), vol. 1, Sun City, South Africa, Sept. 1998, pp. 262 - 266.

[97] S. Min and H. L. Bertoni, "Effect of path loss on cdma system design for highway microcells," in Proc. 48th IEEE Vehicular Technology Conference (VTC'1998), Ottawa, Canada, May 1998, pp. 1009-1013.

[98] C. Ho, J. Copeland, C. Lea, and G. Stüber, "Impact of the cell size on the cell's erlang capacity and call admission control in the ds/cdma cellular networks," in Proc. IEEE Vehicular Technology Conference (VTC'2000), vol. 1, Tokyo, Japan, May 2000, pp. $385-389$.

[99] G. Hernandez-Valdez, F. A. Cruz-Perez, and M. Lara, "Impact of the cell size and the propagation model parameters on the performance of microcellular networks," in Proc. 11 th IEEE International Symposium on Personal, Indoor and Mobile Radio Communications (PIMRC'2000), vol. 1, London,UK, 2000, pp. 292 - 296.

[100] D. Har and H. L. Bertoni, "Effect of anisotropic propagation modelling on microcellular system design," IEEE Trans. Veh. Technol., vol. 49, no. 4, pp. 1303 - 1313, Aug. 2000.

[101] B. T. Ahmed, M. C. Ramon, and L. de Haro Ariet, "Capacity and interference statistics of highways w-cdma cigar-shaped microcells (uplink analysis)," IEEE Commun. Lett., vol. 6, no. 5, pp. 172 - 174, Aug. 2002.

[102] A. Karlsson, R. Schuh, C. Bergljung, P. Karlsson, and N. Lowendahl, "The influence of trees on radio channels at frequencies of 3 and $5 \mathrm{ghz}$," in Proc. 54th IEEE Vehicular Technology Conference (VTS'2001 Fall), vol. 4, Altantic City, New Jersey, USA, Oct. 2001, pp. $2008-2012$.

[103] G. A. Roch, “Channel occupancy time distribution in a cellular radio system," IEEE Trans. Veh. Technol., vol. 40, no. 3, pp. 89 - 99, Aug. 1987. 
[104] S. Ihara, "On the capacity of channels with additive non-gaussian noise," Inform. and Control, vol. 37, pp. $34-39,1978$.

[105] S. Shamai and A. D. Wyner, "Information-theoretic consideration for symmetric cellular, multiple-access fading channels - part i," IEEE Trans. Inform. Theory, vol. IT - 43, no. 11, pp. 1877 - 1894, Nov. 1997.

[106] B. Gudmundson, J. Skold, and J. K. Ugland, "A comparison of cdma and tdma systems," in Proc. 42nd IEEE Vehicular Technology Conference (VTC'1992), vol. 2, Denver, CO, USA, May 1992, pp. 732 - 735.

[107] G. TR25.996, “3gpp scm channel models,”" 3GPP TR25.996, vol. v6.1.0, Sept. 2003.

[108] I. Cuinas and M. G. Sánchez, "Wide-band measurements of nondeterministic effects on the bran indoor radio channel," IEEE Trans. Veh. Technol., vol. 53, no. 4, pp. $1167-1175$, Jul. 2004.

[109] R. Prasad, "Overview of wireless personal communications: Microwave perspectives," IEEE Commun. Mag., vol. 35, no. 4, pp. 104 - 108, Apr. 1997.

[110] P. Smulders, "Statistical characterization of 60-ghz indoor radio channels," IEEE Trans. Antennas Propagat., vol. 57, no. 10, pp. 2820 - 2829, Oct. 2009.

[111] J. H. Whitteker, "Physical optics and field-strength predictions for wireless systems," IEEE J. Select. Areas Commun., vol. 20, no. 3, pp. 515 - 522, Apr. 2002.

[112] C. Chih-Yuan and K. Chen, "Effects of rain fading on the efficiency of the ka-band lmds system in the taiwan area," IEEE Trans. Veh. Technol., vol. 54, no. 1, pp. 9 19, Jan. 2005.

[113] M. Dohler, R. Heath, A. Lozano, C. B. Papadias, and R. A. Valenzuela, "Is the phy layer dead?" IEEE Commun. Mag., vol. 49, no. 4, pp. 159 - 165, Apr. 2011.

[114] E. Telatar, "Capacity of multi-antenna gaussian channels," European transactions on telecommunications, vol. 10, no. 6, pp. 585 - 595, Nov. 1999. 
[115] S. Catreux, P. F. Driessen, and L. J. Greenstein, "Attainable throughput of an interference-limited multiple-input multiple-output (mimo) cellular system," IEEE Trans. Commun., vol. 49, no. 8, pp. 1307 - 1311, Aug. 2001.

[116] R. S. Blum, "Mimo capacity with interference," IEEE Trans. Veh. Technol., vol. 21, no. 5 , pp. $793-801$, Jun. 2003.

[117] K. A. Anang, P. B. Rapajic, R. Wu, L. Bello, and T. I. Eneh, "Impact of propagation/system parameters on cellular wireless network operating at frequencies greater than 2 ghz," in Proc. 8th IEEE International Wireless Communications and Mobile Computing Conference (IEEE-IWCMC 2012), Limassol, Cyprus, Aug. 2012, pp. $1169-1175$.

[118] K. A. Anang, T. I. Eneh, L. Bello, and D. L. Larkai, "The influence of vehicular traffic on cellular wireless system performace at microwave carrier frequencies," in Proc. 4th IEEE International conference on adaptive science \& technology (IEEEICAST 2012), Kumasi, Ghana, Oct. 2012, pp. 17 - 22.

[119] D. Cox, "Cochannel interference considerations in frequency reuse small-coveragearea radio systems," IEEE Trans. Commun., vol. COM-30, no. 1, pp. 135 - 142, Jan. 1982.

[120] J. G. Andrews, "Interference cancellation for cellular systems: a contempory overview," IEEE Trans. Commun., vol. 12, no. 2, pp. 19 - 29, Apr. 2005.

[121] K. Ogawa, A. Yamamoto, and J. Takada, "Multipath performance of handset adaptive array antennas in the vicinity of a human operator," IEEE Trans. Antennas Propagat., vol. 53, no. 8, pp. 2422 - 2435, Aug. 2005.

[122] J. Zander, "On the cost structure of future wideband wireless access," in Proc. 47th IEEE Vehicular Technology Conference (VTC'1997), vol. 3, Arizona, USA, May 1997, pp. $1773-1776$.

[123] D. Astély, E. Dahlman, A. Anders, Y. Jading, M. Lindstrom, and S. Parkvall, "Lte: the evolution of mobile broadband," IEEE Commun. Mag., vol. 47, no. 4, pp. $44-$ 51, Apr. 2009. 
[124] A. Ghosh, R. Ratasuk, B. Mondal, N. Mangalvedhe, and T. Tim, "Lte-advanced: next-generation wireless broadband technology [invited paper]," IEEE Trans. Wireless Commun., vol. 17, no. 3, pp. 10 -22, Jun. 2010.

[125] I. Akyildiz, D. M. Gutierrez-Estevez, and E. C. Reyes, "The evolution to 4g cellular systems: Lte-advanced," Physical Communication, vol. 3, no. 4, pp. 217 - 244, 2010.

[126] A. Khandekar, N. Bhushan, J. Tingfang, and V. Vanghi, "Lte-advanced: Heterogeneous networks," in Proc. 2010 European Wireless Conference (EW'2010), Lucca, Italy, Apr. 2010, pp. 978 - 982.

[127] D. Lopez-Perez, I. Guvenc, G. D. L. Roche, M. Kountouris, T. Q. S. Quek, and J. Zhang, "Enhanced intercell interference coordination challenges in heterogeneous networks," IEEE Trans. Wireless Commun., vol. 18, no. 3, pp. 22 - 30, Jun. 2011.

[128] R. Madan, J. Borran, A. Sampath, N. Bhushan, A. Khandekar, and T. Ji, "Cell association and interference coordination in heterogeneous lte-a cellular networks," IEEE J. Select. Areas Commun., vol. 28, no. 9, pp. 1479 - 1489, Dec. 2010.

[129] N. Himayat, S. Talwar, A. Rao, and R. Soni, "Interference management for 4g cellular standards [wimax/lte update]," IEEE Commun. Mag., vol. 48, no. 8, pp. 86 - 92, Aug. 2010.

[130] T. D. Novlan, R. K. Ganti, A. Ghosh, and J. G. Andrews, "Analytical evaluation of fractional frequency reuse for ofdma cellular networks," IEEE Trans. Wireless Commun., vol. 10, no. 12, pp. 4294 - 4305, Dec. 2011.

[131] H. Zhu and J. Wang, "Frequency reuse in chunk-based multi-cell ofdma systems," in Proc. 2012 IEEE International Conference on Communications, (ICC'2012), Ottawa, ON, Canada, Jun. 2012, pp. 1 - 5.

[132] S.-E. Elayoubi, O. B. Haddada, and B. Fourestie, "Performance evaluation of frequency planning schemes in ofdma-based networks," IEEE Trans. Wireless Commun., vol. 7, no. 5, pp. 1623 - 1633, May 2008. 
[133] Y. Lan and A. Harada, "Interference analysis and performance evaluation on the coexistence of macro and micro/pico cells in lte networks," in Proc. 75th IEEE Vehicular Technology Conference (VTC'2012 - Spring), Yokohama, Japan, May 2012, pp. $1-5$.

[134] T. D. Novlan, "Fractional frequency reuse for multi-tier cellular networks," $\mathrm{PhD}$ dissertation, The University of Texas at Austin, 2012.

[135] J. G. Andrews, F. Baccelli, and R. K. Ganti, “A tractable approach to coverage and rate in cellular networks," IEEE Trans. Commun., vol. 59, no. 11, pp. 3122 - 3134, Nov. 2011. 